\title{
Comprehensive Two-dimensional Gas Chromatography in Environmental Analysis
}

\author{
by \\ Alina Mihaela Muscalu \\ A thesis \\ presented to the University of Waterloo \\ in fulfillment of the \\ thesis requirement for the degree of \\ Doctor of Philosophy \\ in \\ Chemistry
}

Waterloo, Ontario, Canada, 2019

(C) Alina Mihaela Muscalu 2019 


\section{Examining Committee Membership}

The following served on the Examining Committee for this thesis. The decision of the Examining Committee is by majority vote.

External Examiner

Supervisor(s)

Internal Member

Internal-external Member

Other Member(s)
NAME Jean-François Focant

Title Prof. Dr.

NAME Tadeusz Górecki

Title Prof. Dr.

NAME Wojciech Gabryelski

Title Prof. Dr.

NAME Rolland Hall

Title Prof. Dr.

NAME Terry McMahon

Title Prof. Dr. 


\section{AUTHOR'S DECLARATION}

I hereby declare that I am the sole author of this thesis. This is a true copy of the thesis, including any required final revisions, as accepted by my examiners.

I understand that my thesis may be made electronically available to the public. 


\begin{abstract}
Comprehensive two-dimensional gas chromatography $(\mathrm{GC} \times \mathrm{GC})$, a separation method offering greater peak capacity than conventional one-dimensional separations, has been rapidly growing in popularity. The GC $\times \mathrm{GC}$ separation is performed by interfacing a modulator between two columns, where the modulator is referred to as the heart of the GC $\times \mathrm{GC}$ system. It functions by periodically sampling, trapping and re-injecting the effluent from the first column into the second column. The most frequently used $\mathrm{GC} \times \mathrm{GC}$ systems require consumables such as liquid $\mathrm{N}_{2}$ for the trapping function of the modulator. Although these systems are very effective, their initial and running costs are a hindrance to more widespread use. A new, single-stage thermal modulator for $\mathrm{GC} \times \mathrm{GC}$, that requires no consumables for operation has been developed to overcome these problems. In this work, the system was evaluated and compared to a routine accredited method for the analysis of polychlorinated biphenyls, organochlorine pesticides and chlorobenzenes was run using the new modulator and its performance was compared to that of an industry standard modulation system. Replicate analyses of these samples have been performed and within-day repeatability, between-day reproducibility, as well as between-trap reproducibility were assessed. The results are very encouraging as negligible shifts in retention times were observed for both within-day and dayto-day comparisons of the studied samples, and the quantitative results were comparable. Routine analysis and quality control applications will also benefit from improved retention time reproducibility. An overview of the device operation and the results from this study are summarized.
\end{abstract}


With thousands of chemicals routinely used on a daily basis, the need for multiresidue methods to prioritize the monitoring of potentially persistent, bioaccumulating and toxic contaminants is increasing. $\mathrm{GC} \times \mathrm{GC}$ is known to be one of the most powerful techniques for the separation of complex environmental samples, especially for the analysis of compound groups that contain congeners or homologues like polychlorinated biphenyl congeners (PCBs). An analytical method was developed by using GC $\times \mathrm{GC}$ coupled with micro-electron capture detector $(\mu \mathrm{ECD})$ to separate and quantify short chain chlorinated paraffins (SCCP) in sediment samples. Chlorinated paraffins (CPs) are complex mixtures of chlorinated n-alkanes with varying chain lengths $\left(\mathrm{C}_{10}\right.$ to $\left.\mathrm{C}_{30}\right)$ and degree of chlorination (30\% to $70 \%$ by weight). Distinct ordered bands were observed in the $\mathrm{GC} \times \mathrm{GC}$ chromatograms pointing to group separation. Using the Classification function of the ChromaTOF software, summary tables were generated to determine total area counts to set up multilevel-calibration curves for different technical mixes. Fortified sediment samples were analyzed by $\mathrm{GC} \times \mathrm{GC}-\mu \mathrm{ECD}$ with minimal extraction and cleanup. Recoveries ranged from $120 \%$ to $130 \%$. To further validate the proposed method for the analysis of SCCPs, the laboratory participated in inter-laboratory studies for the analysis of standards and sediment samples. The results showed recoveries between $75 \%$ to $95 \%$ and z-score values $<2$, demonstrating that the method is suitable for the analysis of SCCPs in soil/sediment samples.

In a different study, hydroxylated polychlorinated biphenyls (OH-PCB) were discovered in water samples collected from storm sewers located in southwestern Ontario. Requiring minimal extraction and cleanup procedures, the samples were analyzed by $\mathrm{GC} \times \mathrm{GC}$ coupled with micro-electron capture detection. "Unknown" peaks observed in the two 
dimensional chromatograms were further investigated by $\mathrm{GC} \times \mathrm{GC}$ coupled with time-of-flight mass spectrometry (TOFMS) and tentatively identified as OH-PCBs. Mass spectral data and further derivatization confirmed the presence of dichloro- to pentachloro-mono-OH-PCBs. Hydroxylated PCB standards for different chlorination levels were multi-level calibrated using GC $\times$ GC-TOFMS and a semi-quantitative procedure was applied for each peak identified as OH-PCB in the samples. Preliminary results for the total di-, tri-, tetra- and pentachlorohydroxylated PCBs showed higher levels for these compounds than any reported so far in environmental samples.

Another analytical method was developed by using GC×GC- $\mu$ ECD and -TOFMS to identify and quantify polychlorinated diphenyl ethers (PCDEs) in sediment samples. Following up the tentative identification of PCDEs by GC $\times$ GC- $\mu$ ECD, GC $\times$ GC-TOFMS was further used for quantitative and semi-quantitative analysis. As a result, mono- to nonachlorinated diphenyl ethers were detected and quantified in sediment samples collected from Lake Ontario. The levels of PCDEs in the samples ranged from non-detects to $8000 \mathrm{ng} / \mathrm{g}$. When positive results were reported, it was observed that mono- to tri-CDE had a higher contribution, greater than $30 \%$, towards the total PCDE amount.

The findings convincingly demonstrate that using a technique such as $\mathrm{GC} \times \mathrm{GC}-\mu \mathrm{ECD}$ for the routine testing of environmental samples is an excellent screening approach for detecting non-targeted contaminants. 


\section{Acknowledgements}

First, I would like to thank my supervisor, Dr. Tadeusz Górecki for his patient guidance, expertise and constructive feedback throughout the term of my PhD Program and my thesis projects. With his vast knowledge and enthusiasm, approachable personality and an always open office door, Prof. Górecki has truly inspired me to never give up and that anything is possible.

I would like to express my gratitude to Dr. Eric Reiner and Dave Morse, whose continuous support and encouragement kept me focused and on track with the projects' work. Their guidance throughout the years has strengthened my confidence in my abilities and has increased my passion for the work I am doing.

My appreciation also goes to the Ministry of Environment, Conservation and Parks staff for encouragement and help with setting up the experiments. Vedrana Pantelic, Stephanie Lemanik, Terry Kolic, Roxana Banaru and Chris Schneider, you have made my work a lot easier and your help is appreciated. Many thanks to Jennifer Sifton from Fisheries and Oceans Canada for providing the sediment core samples for one of the projects.

Many thanks to Jack Cochran for his advice and help in providing me with anything required to build my experimental setups and make them work. I would also like to thank K. C. Wallbanks from LECO for always being there, advising and helping me bring the instruments back on-line.

I would like to thank my high school teachers Elena Varga and Carmen Tanasescu; their passion for teaching chemistry and physics has inspired me to pursue a career in science. 
Finally, I would like to thank my family for their never-ending support, motivation and inspiration; my lovely kids Andreea and Daniel, husband Adrian and my parents: your patience during all the years of "mom's working" times is greatly appreciated. No words can express my gratitude, I am very lucky to have you in my life. Many thanks to my friends Carmen Gaspar and Gabriela Horga for your encouragement and support when things were not going as planned. 


\section{Dedication}

This work is dedicated to my mom

\section{Dorina Moldovan}

May her memory forever be a comfort and a blessing.

She was my best friend, my support, the best mother anyone can ever have. 


\section{Table of Contents}

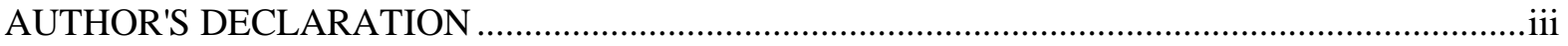

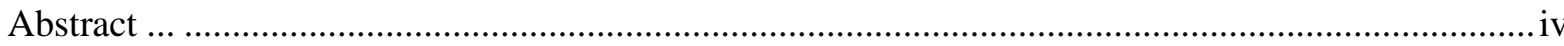

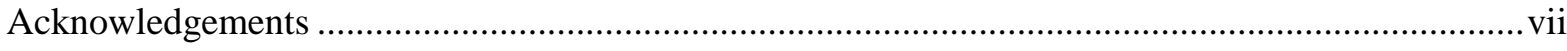

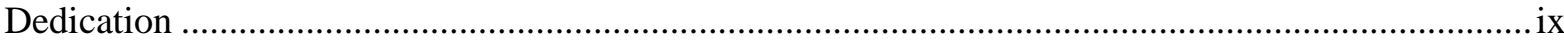

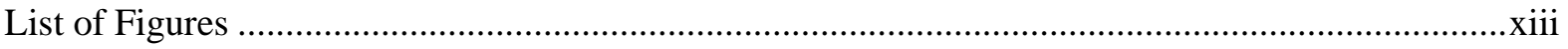

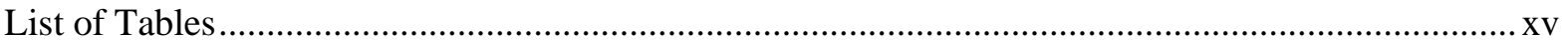

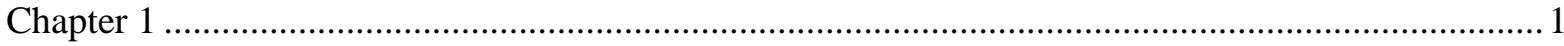

1.1 Comprehensive Two-dimensional Gas Chromatography - Basic Concepts and Set-up.............2

1.2 Comprehensive Two-dimensional Gas Chromatography in Environmental Analysis ................ 9

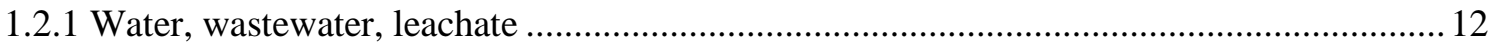

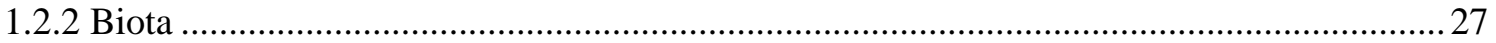

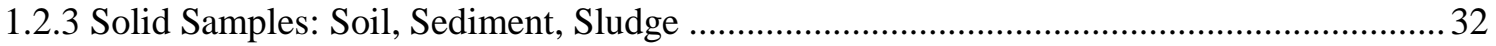

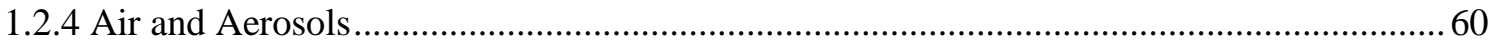

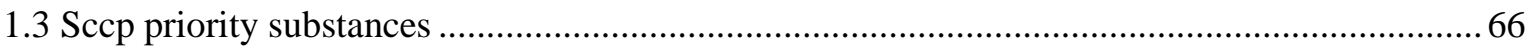

1.4 Halogenated organic contaminants in environmental samples.............................................66

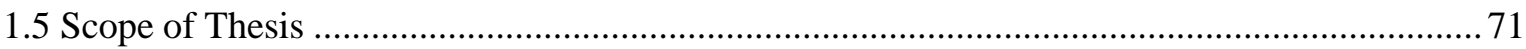

Chapter 2 Evaluation of a Single-Stage Consumable-Free Modulator for GC×GC: Analysis of

Polychlorinated Biphenyls, Organochlorine Pesticides and Chlorobenzenes........................ 73

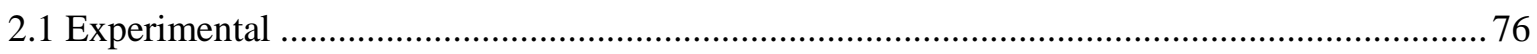

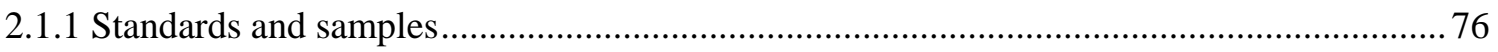

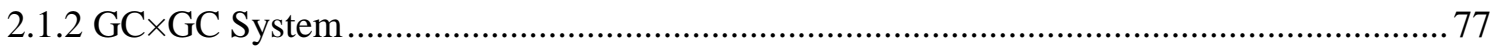

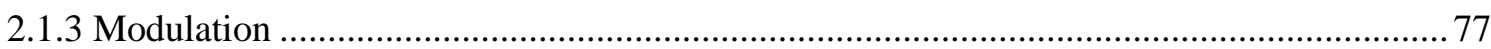

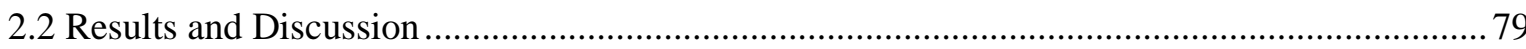

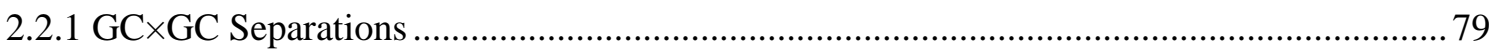

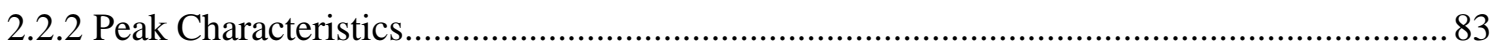

2.2.3 Thermal Modulator Performance: Quantification, Repeatability and Reproducibility .......85

2.2.4 Robustness - between-run and between-trap system reproducibility............................... 91

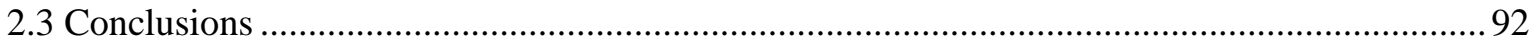

Chapter 3 The Quantification of Short Chain Chlorinated Paraffins in Sediment Samples Using

Comprehensive Two-Dimensional Gas Chromatography with $\mu$ ECD Detection ................93

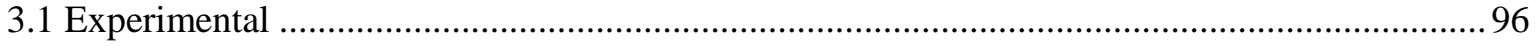




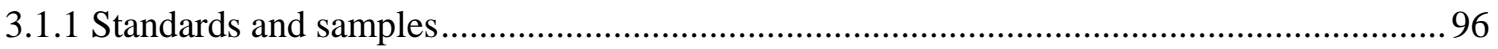

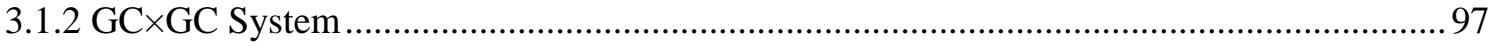

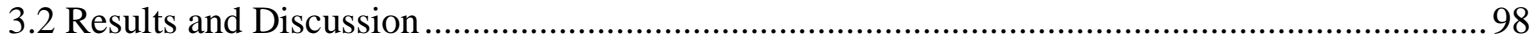

3.2.1 Short Chain Chlorinated Paraffins Separation.................................................................... 98

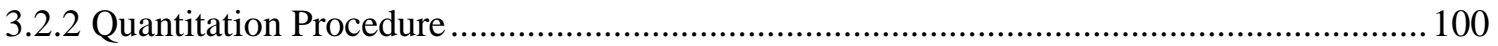

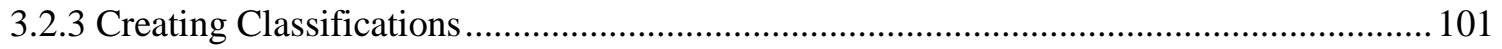

3.2.4 Calibration Procedure and Instrument/Method Performance ............................................ 104

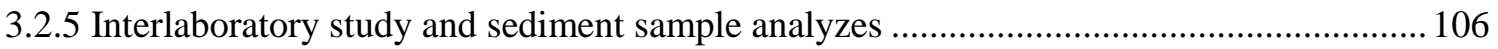

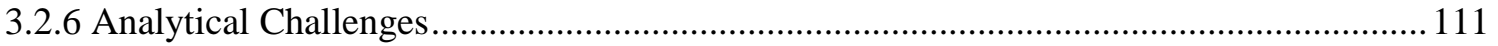

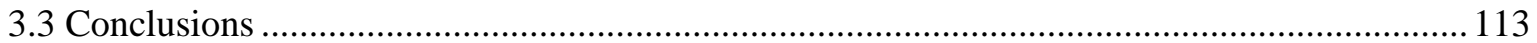

Chapter 4 Non-targeted Analysis of Hydroxylated Polychlorinated Biphenyls in Storm Sewer Water

Using Comprehensive Two-Dimensional Gas Chromatography with Micro-electron Capture Detection and Time-of-flight Mass Spectrometry .......................................................... 114

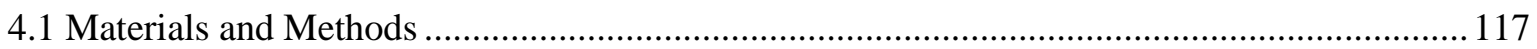

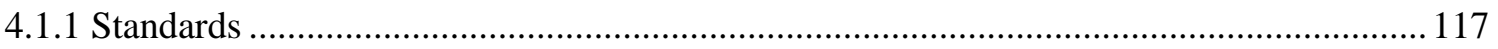

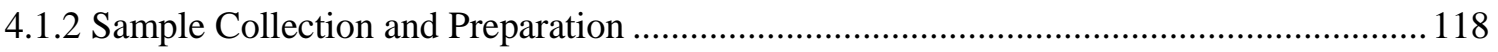

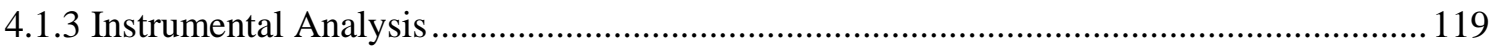

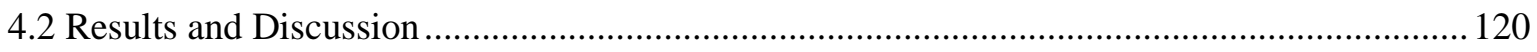

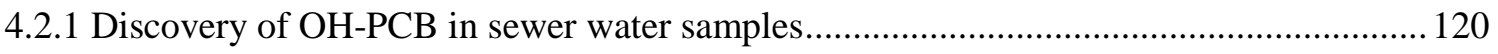

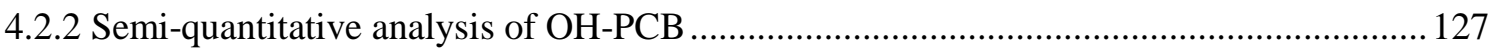

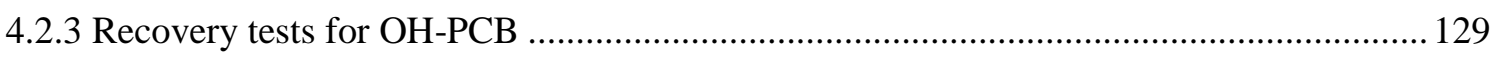

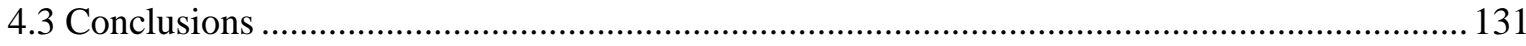

Chapter 5 The Analysis of Polychlorinated Diphenyl Ethers in Sediments by Comprehensive Two-

Dimensional Gas Chromatography with Micro-electron Capture Detection and Time-of-

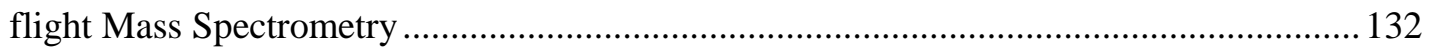

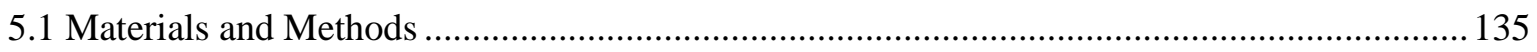

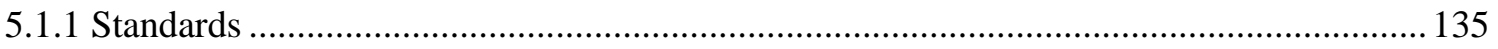

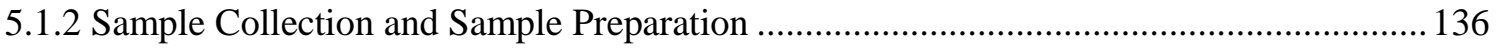

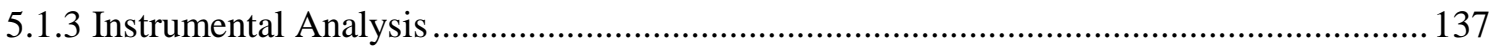

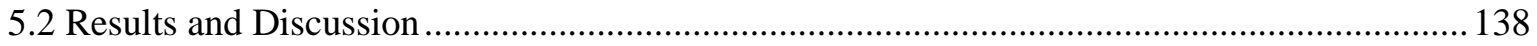

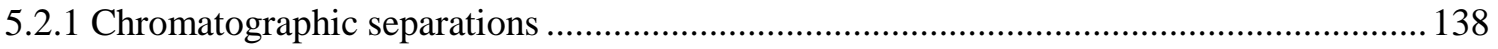

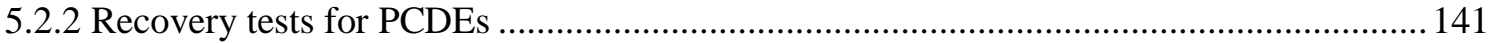


5.2.3 Quantitative and semi-quantitative analysis of PCDEs in sediments samples

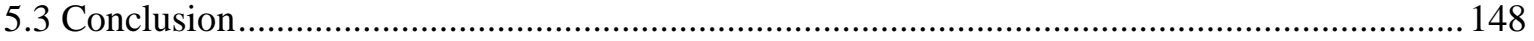

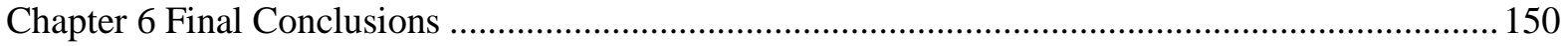

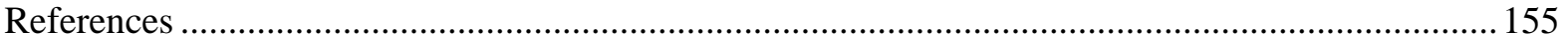

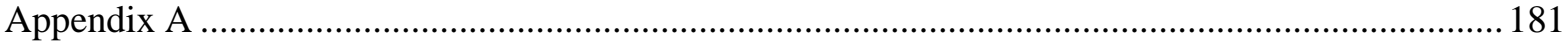

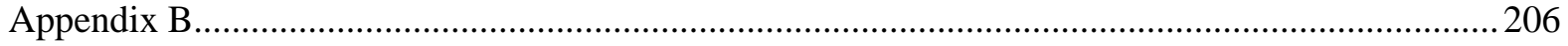

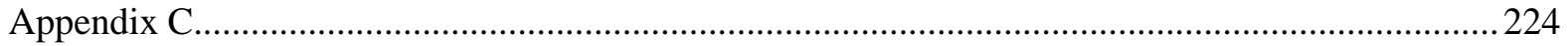

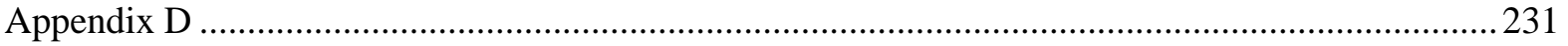




\section{List of Figures}

Figure 1.1 Schematic of two-oven GCXGC system and explanation of cryo-modulation....... 3

Figure 1.2 Flow path of a differential flow modulator ................................................. 7

Figure 1.3 Comparison of o,p_-/p,p_-DDD and p,p_-DDT separation using the two-

dimensional mass chromatogram (m/z 235.0083) obtained by SBSE-TD-GC $\times$ GC-

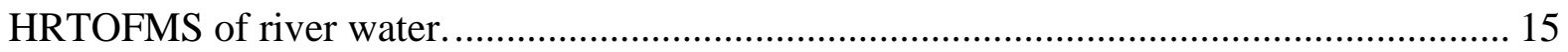

Figure 1.4 2D contour plot of landfill leachate analyzed by SDE-GC $\times$ GC/TOFMS ............ 19

Figure 1.5 Contour plot of a reconstructed ion chromatogram (RIC) (GC $\times$ GC-TOFMS) of

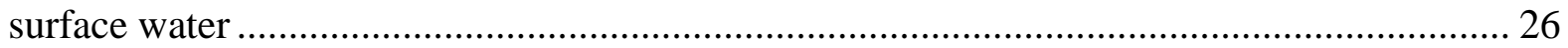

Figure 1.6 Example of a GC×GC-TOFMS chromatogram of PAHs separated on

BPX5×BPX50 column system.

Figure 1.7 Comparison of group type separation using the 2D mass chromatograms obtained using the $\mathrm{GC} \times \mathrm{GC}-\mathrm{HRTOFMS}$ of a soil extract.

Figure 1.8 Chromatograms of the molecular ions obtained from $\mathrm{GC} \times \mathrm{GC}-\mathrm{HRTOF}$ analysis.

The structures are tentatively proposed on the basis of exact mass measurements and EI

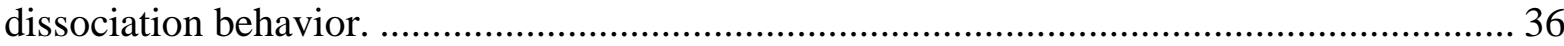

Figure 1.9 Comparison of the results obtained from different data processing methods. ..... 43

Figure 1.10 Sludge and sediment samples analyzed by $\mathrm{GC} \times \mathrm{GC}$ showing the presence of

polychlorinated terphenyls along with the method's target compounds

Figure1.11 GC $\times$ GC-FID contour plots of 100 ppm FAME and BAME mixtures on GC

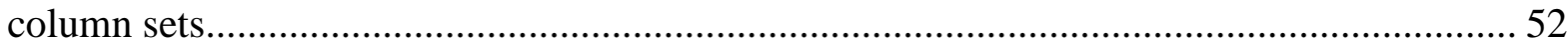

Figure $1.12 \mathrm{GC} \times \mathrm{GC}-\mathrm{ECNI}-\mathrm{HRTOF}-\mathrm{MS}$ chromatograms and $\mathrm{GC} \times \mathrm{GC}$ elution pattern for a technical 51.5\% Cl SCCPs mixture and a technical $52 \% \mathrm{Cl}$ MCCPs mixture.................... 56

Figure1.13 Reconstructed contour plot for sample ....................................................... 59

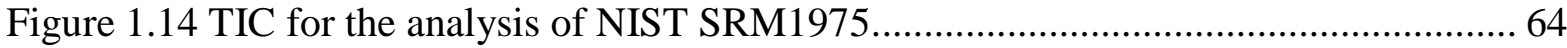

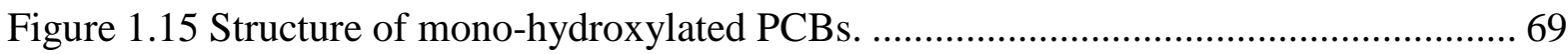

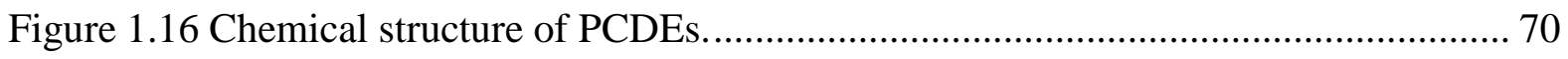

Figure 2.1 Single-stage, consumable-free thermal modulation system. .............................. 78 
Figure $2.2 \mathrm{GC}$ x GC- $\mu \mathrm{ECD}$ contour plot of $\mathrm{PCB} / \mathrm{OC} / \mathrm{CB}$ standard mix using a consumablefree modulator and LN2 modulator 81

Figure $2.3 \mathrm{GC} \times \mathrm{GC}-\mu \mathrm{ECD}$ contour plot of co-elutions when using the quad-jet modulator and (B) resolving co-elutions when using the new consumable-free thermal modulator....... 83 Figure 2.4 Peak height comparison for selected compounds analyzed with LN2 modulator

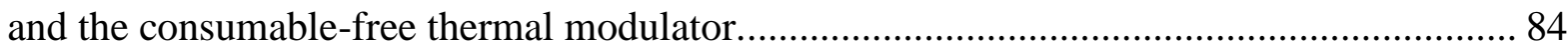

Figure 2.5 Sediment Reference Material (SRM1944) Comparison: ........................................ 90

Figure $3.1 \mathrm{GC} \times \mathrm{GC}-\mu \mathrm{ECD}$ chromatograms of SCCP by using DB1 x Rtx-PCB ................. 100 Figure 3.2 The representation of the Classifications created for SCCP $55.5 \% \mathrm{Cl}$ mixture

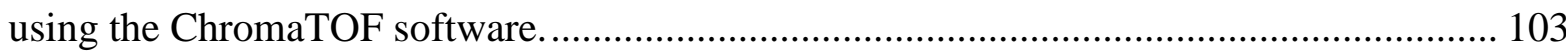

Figure 3.3 Quasiememe Phase I - GC×GC- $\mu$ ECD chromatograms ..................................... 108

Figure 3. 4 Quasiememe Phase III - GC×GC- $\mu$ ECD chromatograms................................. 110

Figure 3.5 Overlaid retention times of $\mathrm{PCB} / \mathrm{OC} / \mathrm{CB}, \mathrm{SCCP}$ and MCCPs at different

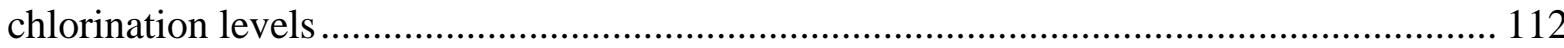

Figure 4.1 Aroclor profiles for the three water samples ...................................................... 122

Figure 4.2 The representation of 2D chromatograms and mass spectra for water sample \#2

Figure 4.3 Selected ion chromatograms for tri-chlorinated and tetra-chlorinated compounds before and after derivatization. 126

Figure 5.1 Two-dimensional chromatograms representing the separation of $\mathrm{PCB} / \mathrm{OC} / \mathrm{CB}$,

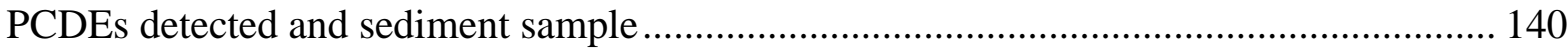

Figure 5.2 PCDE recovery tests for sample preparation procedure …………………........ 142

Figure 5.3 Mass spectra of two tetrachlorodiphenyl ethers: PCDE 74 and PCDE 77 .......... 144

Figure 5.4 Sediment samples analyzed by GC×GC-TOFMS:.............................................. 147 


\section{List of Tables}

Table 1.1 Advantages and disadvantages of thermal and flow modulators.......................... 8

Table 2.1 Peak width attributes: Full Width at Half Height as obtained with both modulation

systems

Table 2.2 Instrument Detection Limits for the GC $\times$ GC- $\mu$ ECD systems with the two

modulators 87

Table 2.3 Repeatability - within-run standards at $20 \mathrm{ng} / \mathrm{mL}$ : PCBs with different chlorination

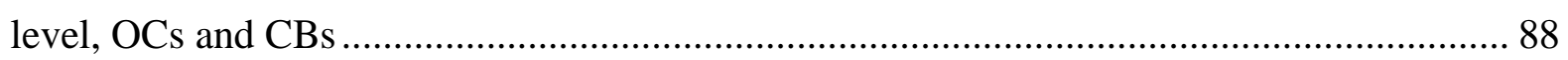

Table 3. 1 The representation of the classification summary for SCCP with different chlorination level $(200 \mathrm{ng} / \mathrm{mL})$ 102

Table 3.2 Response factors for different CP isomers 105

Table 3.3 SCCP 55.5 \% chlorine - Multilevel-calibration Standards and Spike Recoveries 106

Table 4.1 Semi-quantitation of OH-PCBs for the storm sewer overflow water samples 128

Table 4.2 Recovery tests for OH-PCBs for the extraction and clean-up procedures 130

Table 5.1 Levels of PCDEs in a sediment sample analyzed by GC×GC-TOFMS 146 


\section{Chapter 1}

\section{Introduction $^{\mathrm{i}}$}

First introduced by John Phillips in 1991 [1], comprehensive two-dimensional gas chromatography $(\mathrm{GC} \times \mathrm{GC})$ is a powerful technique that provides significant increases in separation power, peak capacity, and sensitivity. Due to its proven benefits over the one dimensional gas chromatography (1D-GC), GC $\times$ GC gained popularity over the last two decades in various research fields. There are many excellent reviews published over the years discussing the theory of and advances in $\mathrm{GC} \times \mathrm{GC}$, with some of them focusing on selected aspect of the technique, including modulators, detectors and data processing. This introductory section provides a brief overview of the technique and focuses on the applications of the $\mathrm{GC} \times \mathrm{GC}$ in environmental analysis. In addition, physical and chemical properties, as well as the environmental occurrence and significance of the organic halogenated compounds presented in this research are discussed.

In environmental testing, GCxGC has been applied successfully in many studies for the detection of contaminants such as pesticides, pharmaceuticals, disinfection by-products, hydrocarbons and synthetic musks in water, biota, air, and solid samples, particularly in drinking water samples where concentration limits are set by regulations. $\mathrm{GC} \times \mathrm{GC}$ analyses yielded more information on the presence and levels of different groups of contaminants, provided additional selectivity for more accurate detection, helped with compound class visualization and with

\footnotetext{
i This chapter is partially based on the author's paper, A.M. Muscalu and T. Górecki, "Comprehensive Twodimensional Gas Chromatography in Environmental Analysis", TrAC, 106 (2018) 225-245²
} 
identification of minor compounds in complex environmental samples. Modern applications in the environmental field emphasized the advantages of the technique in non-targeted analysis in complex matrices, and helped track the contamination source, perform risk assessment, or improve different treatment processes [2].

\subsection{Comprehensive Two-dimensional Gas Chromatography - Basic Concepts and Set-up}

Conventional gas chromatography (GC) offers reasonably good peak capacity, but it fails to separate many individual constituents in complex samples. The selectivity and peak capacity of GC $\times \mathrm{GC}$ are increased through the application of two independent separation mechanisms to a sample in a single analysis, resulting in improved resolution of target compounds from structurally similar compounds and matrix interferences. Over the years, multiple reviews of GC $\times$ GC have been published describing both the principles and instrumental set-ups [3-9]. More recently, the focus of the $\mathrm{GC} \times \mathrm{GC}$ research was shifted towards modulation [6-7], detection [8-9] and real-life applications.

GC $\times \mathrm{GC}$ involves a serial column configuration interfaced by a modulator. It is a truly comprehensive technique as the entire sample is separated on two columns with different selectivities, and the separation accomplished in the first dimension is preserved in the second one. The general schematic of a GC×GC system is illustrated in Figure 1.1, showing the first dimension capillary column connected through an interfacing device (modulator) to the second dimension capillary column, which is in turn connected to the detector. Depending on the particular implementation of the system, the columns can be housed in a single oven, or two independently controlled ovens [4]. Because the columns are connected in a single train, many 
parameters such as temperature programs, flow rates, column dimensions (lengths and internal diameter) are interdependent [10].

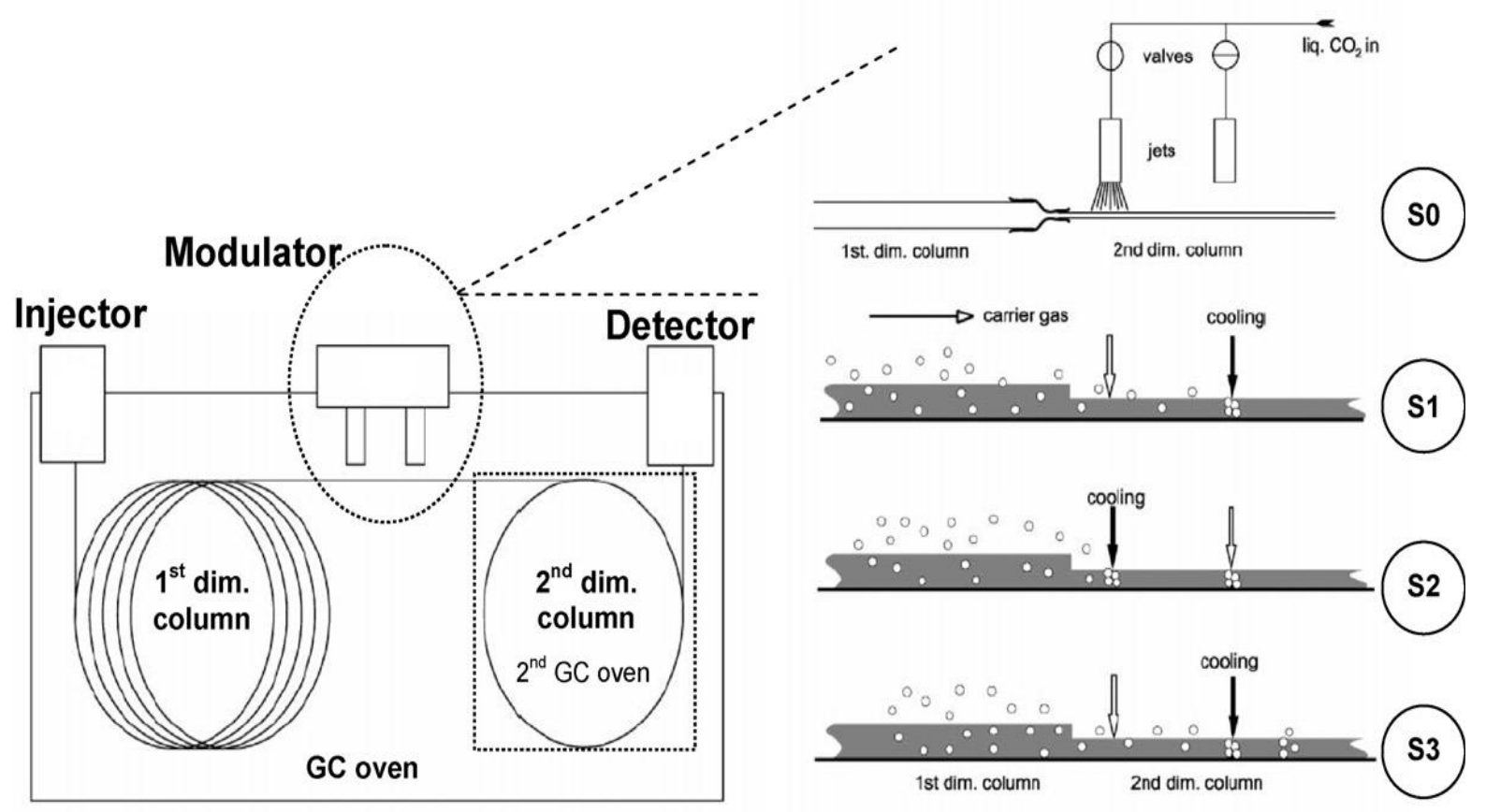

Figure 1.1 Schematic of two-oven GC $\times$ GC system and explanation of cryo-modulation. Left: typical set-up. Right: (S0) general set-up of dual-jet cryogenic modulator. (S1) Right-hand-side jet traps analytes eluting from first-dimension column; (S2) right-hand-side jet switched off, cold spot heats up rapidly and analyte pulse is released into second-dimension column; simultaneously, left-hand-side jet switched on to prevent leakage of first-dimension column material; (S3) next modulation cycle is started. Partially reproduced from Ref. [4], Copyright (2008), with permission from Elsevier.

The first dimension $\left({ }^{1} \mathrm{D}\right)$ column in GC $\times \mathrm{GC}$ is typically quite long (15-60 m, usually nonpolar for a typical set-up). Consequently, the separation time in ${ }^{1} \mathrm{D}$ (which is also the overall separation time) is quite long (45 to $120 \mathrm{~min}$ ). The second dimension $\left({ }^{2} \mathrm{D}\right)$ column is generally polar or shape-selective in nature, much shorter $(0.5-2 \mathrm{~m})$ and often narrower than the first 
column. The separation in ${ }^{2} \mathrm{D}$ is very fast (1-10 s) and results in very narrow peaks (typically 100 $600 \mathrm{~ms}$ at the base) that require very fast detectors in order to reconstruct the two-dimensional chromatograms properly [11]. To achieve orthogonal separation in a GCXGC system, the columns selected should provide independent separation mechanisms, so that the entire 2D plane is available for peak separation [4]. One of the benefits of orthogonal separations is that ordered structures are observed in the 2D chromatograms for structurally related compounds (homologues, congeners, isomers). This is a very valuable piece of information for group-type identification in both targeted and non-targeted analysis. Other approaches, such as using polar×non-polar or ionic liquids column combinations, have also been used successfully. For specific applications, superior within- and between-class separations were observed with these types of reversed set-ups $[3,11]$.

The GC $\times$ GC separation is performed by interfacing a modulator between two columns, where the modulator is referred to as "the heart" of the $\mathrm{GC} \times \mathrm{GC}$ system. Its main functions are to periodically sample, trap and re-inject the effluent from the first column into the second column for further separation. Modulation must occur at precisely timed, user-defined intervals throughout the analysis (typically between 2 and 10 seconds). The length of the interval is called the modulation period. Over the years, many types of modulation devices have been developed and excellent review articles discussed their history and development [3-4,6-7,12]. Currently, various GCxGC systems are commercially available from several vendors. They are based on two primary modulation techniques classified as either thermal or flow.

Thermal modulators rely on low temperatures to trap the analytes eluting from the first column, and rapid heating to introduce them to the second column. Three general types of thermal modulators were developed over the years: resistively heated trap, rotating thermal modulator 
("sweeper"), and cryogenic, which can be sub-divided into longitudinally movable trap and jet trap. The sweeper consisted of a thick-film capillary used to retain and focus the analytes from the ${ }^{1} \mathrm{D}$ column. Their re-injection into the second column was achieved by a rotating slotted heater which locally heated the capillary column. The main disadvantages of this type of device were the need to move very close to the fragile capillary column and the requirement of high temperature differences [11].

The most commonly used interface is the dual stage quad jet modulator which operates on the principle of sampling, focusing and injecting ${ }^{1} \mathrm{D}$ column effluent into the ${ }^{2} \mathrm{D}$ column using alternately activated hot and cold jets. The cold jets direct cooled nitrogen gas onto a segment of a column, causing the analytes to be trapped in the stationary phase. First cold jet traps analytes eluting from ${ }^{1} \mathrm{D}$ column, then the jet is switched off and the cold spot heats up rapidly resulting in the release of the trapped analyte band into the ${ }^{2} \mathrm{D}$ column for further separation. Simultaneously, the second cold jet is switched on to prevent leakage of first-dimension column material. The two trap and release stages are designed to overcome the breakthrough and the desorption issues. Another device, a commercially available loop type modulator, uses a single cold and hot jet: one jet traps and the other releases the eluate, which travels through the loop where it is trapped again, then desorbed into the ${ }^{2} \mathrm{D}$ column. A single-stage modulator having similar performance to the quad jet modulator was also developed, resulting in savings in the consumption of cryogens [13]. Liquid nitrogen is typically used as the cooling agent for the cold jets; however, consumable-free systems which utilize deep refrigeration units to cool an in-house nitrogen supply gas that feeds the cold jets are also available. These consumable-free systems suffer from sub-par performance compared to their cryogenic alternatives for volatile analytes [67,12]. Other cryogen-free modulators, e.g. Solid State Modulator (SSM) using thermoelectric 
cooling, mica-thermic heating and a movable capillary column, have been developed and used successfully in different applications. The SSM modulator generates narrow peaks $(\leq 120 \mathrm{~ms})$ and is designed to minimize the breakthrough; however, the analysis is dependent of the modulation column used (three listed for different compounds analyzed) [3,12].

Flow modulators use pneumatic means to achieve the modulation of the primary column effluent. Depending of the flow direction, the modulator can either work as a forward fill/flush modulator (FFF) if the injection is in the same direction as the original fill stage, or reversed fill/flush modulator (RFF) if the injection is made in the opposite direction (see Figure 1.2). The breakthrough in flow modulation was introduced by Agilent Technologies, who developed a flow modulator based upon their Capillary Flow Technology [12,14-15]. The setup uses a planar flow splitter and a collection channel interfaced between the primary and the secondary columns, and a three-way solenoid valve that delivers carrier gas to two inputs on the planar device. By switching the flow of the carrier gas between these two inputs, the device operates in a collect state or a flush state. During the collect state, ${ }^{1} \mathrm{D}$ effluent is allowed to enter the device and move through the small volume internal collection channel that fills fast. Once full, the three-way solenoid valve is activated and the auxiliary carrier gas flow is switched to the second input. This purges the collection channel into the secondary column with high carrier gas flow $(20 \mathrm{~mL} / \mathrm{min})$, allowing efficient transfer of the analytes from the collection channel to the second dimension column and physically compressing the chromatographic band. The CFT flow modulator is not limited by sample volatility providing efficient collection and injection of solutes across the entire volatility range. The drawback of this device is the high flow in the second dimension that makes the use of mass spectrometry detection with this technique difficult $[3,6-7,10,12]$. Other devices were also developed, e.g. SepSolve has introduced the INSIGHT modulator, a valve-based 
reverse fill/flush device that facilitates the use of MS detector due to its reduced flow rate in ${ }^{2} \mathrm{D}$ [12]. More recently, LECO has introduced the FLUX modulator, a diverting flow device with a very simple, easy to setup and use design. This device is a cost effective option for GC $\times \mathrm{GC}$; however, the sensitivity is significantly decreased compared to the quad jet thermal modulator [16].
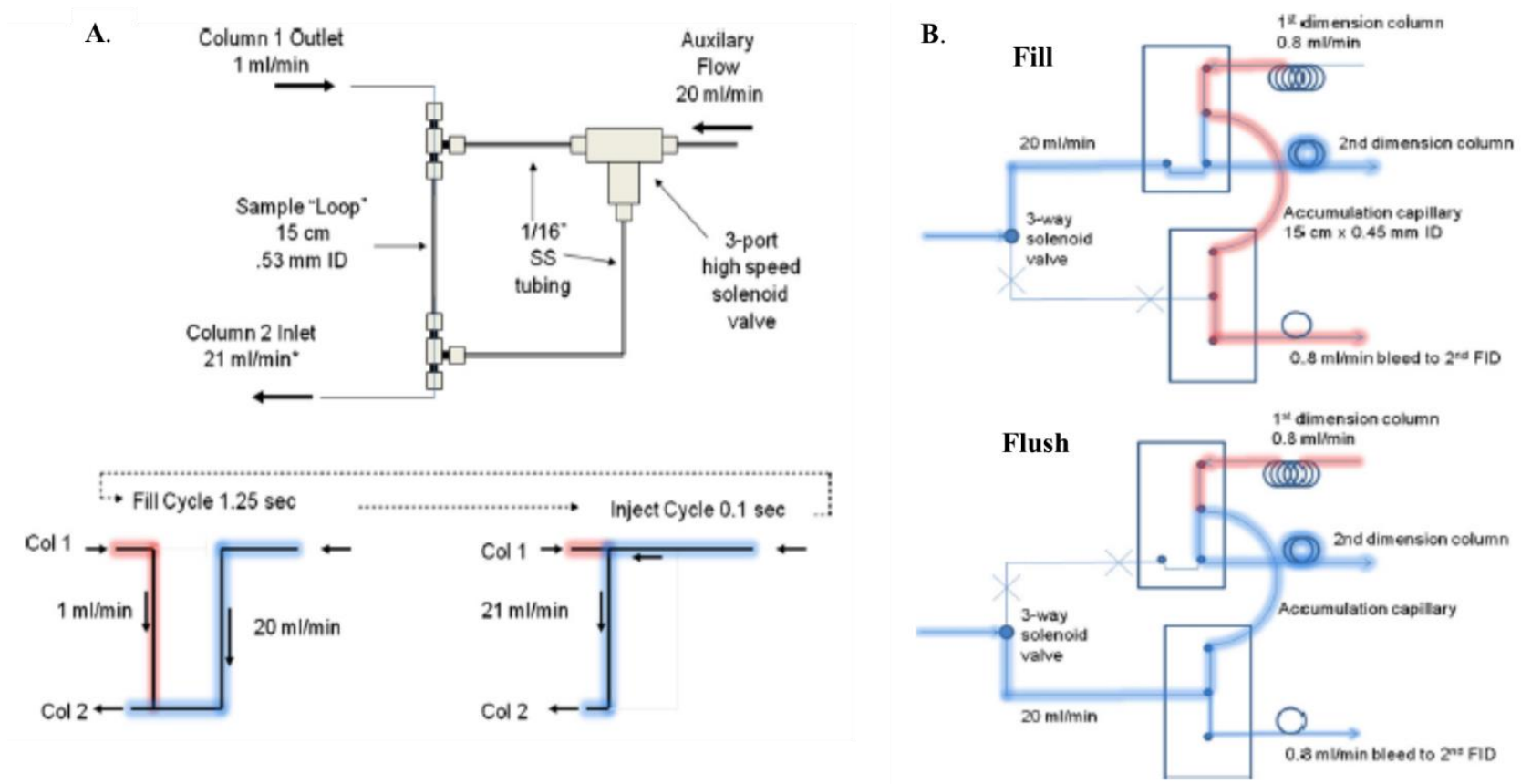

Figure 1.2 Flow path of a differential flow modulator with A) forward fill/flush (FFF), and B) reverse fill/flush (RFF) modulator: flow path of fill cycle (top) and flow path of flush cycle (bottom) [Reprinted from Ref. [14], Copyright (2012), with permission from Elsevier].

A recent review summarized the advantages and disadvantages of both thermal and flow modulators. They are presented in Table 1.1 [12]. 
Table 1.1 Advantages and disadvantages of thermal and flow modulators

\begin{tabular}{|c|c|}
\hline Thermal Modulators & Flow Modulators \\
\hline $\begin{array}{l}\text { Robust, universal, reliable, reproducible, no } \\
\text { moving parts }\end{array}$ & $\begin{array}{l}\text { Some devices (e.g. CFT) proved to be very } \\
\text { reliable and reproducible }\end{array}$ \\
\hline $\begin{array}{l}\text { Complex devices, more expensive, cryogenic } \\
\text { modulators use cryogens that are expensive } \\
\text { and require larger lab space }\end{array}$ & Simpler devices, lower cost \\
\hline Wide applicability $\left(\mathrm{C}_{4}-\mathrm{C}_{40+}\right)$ & Wide applicability $\left(\mathrm{C}_{4}-\mathrm{C}_{40+}\right)$ \\
\hline $\begin{array}{l}>\mathrm{C}_{10} \text { better modulated by cryogenic based } \\
\text { modulators as compared to flow modulators }\end{array}$ & $\begin{array}{l}<\mathrm{C}_{10} \text { superior modulation using the differential } \\
\text { flow modulator }\end{array}$ \\
\hline $\begin{array}{l}\text { Cryogenic-free modulators similar } \\
\text { performance to the cryogenic ones. } \\
\text { Drawbacks: } \mathrm{C}_{3}-\mathrm{C}_{7} \text { challenging to modulate, } \\
\text { some devices require longer modulation } \\
\text { periods }\end{array}$ & \\
\hline High duty cycles (1.0) & $\begin{array}{l}\text { High duty cycles }(>0.5) \text {. Duty cycles }<0.5 \text { in } \\
\text { some applications resulting in loss of sensitivity }\end{array}$ \\
\hline Narrow injection pulse & $\begin{array}{l}\text { Injection pulse width is dominated by the } \\
\text { amount of time the flow is directed from }{ }^{1} \mathrm{D} \text { to } \\
{ }^{2} \mathrm{D}\end{array}$ \\
\hline \multicolumn{2}{|l|}{ Fast modulation period $\left(\mathrm{P}_{\mathrm{M}}>250 \mathrm{~ms}\right)$} \\
\hline High detection sensitivity & $\begin{array}{l}\text { RRF devices showed improved sensitivity and } \\
\text { peak capacity compared to FFF and } \mathrm{CO}_{2} \\
\text { modulators }\end{array}$ \\
\hline Mass spectrometry compatible & $\begin{array}{l}\text { High flow rates on the }{ }^{2} \mathrm{D} \text { column, compatibility } \\
\text { issues with mass spectrometry detection }\end{array}$ \\
\hline $\begin{array}{l}\text { Breakthrough is observed when analyte not } \\
\text { fully trapped }\end{array}$ & \\
\hline
\end{tabular}




\begin{tabular}{|l|l|}
\hline Thermal Modulators & Flow Modulators \\
\hline $\begin{array}{l}\text { Incomplete desorption resulting in wider or } \\
\text { tailing peaks on the }{ }^{2} \mathrm{D} \text { column }\end{array}$ & \\
\hline
\end{tabular}

The narrow injection bands produced by the modulator make very fast separations possible and result in narrow peaks that require fast detectors with a small internal volume, a short detector rise time and a high data acquisition rate for proper reconstruction of the seconddimension chromatograms [11]. The increased resolving power of $\mathrm{GC} \times \mathrm{GC}$ and the generation of structured chromatograms allows more economical detectors, such as electron capture detectors (ECDs) or flame ionization detectors (FIDs), to be used routinely with increased confidence. These detectors offer peak recognition and can be selective (e.g. ECD), but they do not provide structural information. Consequently, the use of mass spectrometric detectors is critical for the identification of the separated compounds. The latest advances in mass spectrometry (MS) technology made the combination of $\mathrm{GC} \times \mathrm{GC}$ hyphenated with different MS systems, such as quadrupole- (Q), time-of-flight (TOF), high-resolution (HR)- time-of-flight (TOF)-, triple quadrupole (QqQ)-, and QTOF-MS possible, adding another analytical dimension to the separation and making the technique more accessible [3,5,8-9]. The use of $\mathrm{GC} \times \mathrm{GC}$ hyphenated to universal or selective detectors for environmental analysis proved to have an outstanding potential for the development of multiresidue methods.

\subsection{Comprehensive Two-dimensional Gas Chromatography in Environmental}

\section{Analysis}

The analysis of persistent organic pollutants in environmental matrices is very challenging due to the large number of compounds with varying chemical and physical properties that are 
typically present in the sample at the same time at concentrations ranging from ultra-trace to percent levels. GC $\times \mathrm{GC}$ is steadily gaining in popularity in environmental analysis and the number of publications citing the use of this technique has been increasing significantly in the recent years. An overview of the latest applications in the environmental field is presented in this paper, emphasizing the advances in targeted and non-targeted analysis in complex matrices. In addition, instrumentation, data interpretation approaches, as well as quality assurance and control for routine analyses are discussed.

Many organic compounds have been and continue to be produced in large volumes for a wide variety of applications, such as agricultural, industrial, household, pharmaceutical, flame retardant, and other uses. Many of these compounds have entered the air, water, and soil during their manufacture, use and disposal. Accidental releases occur, e.g. as a result of chemical spills, fires involving different products, and fugitive emissions. Analysis of environmental samples is very complex due to the large number of compounds with varying chemical and physical properties typically present in the samples at concentrations ranging from ultra-trace to percent levels. The quantitation of the target compounds is not an easy task for the analytical chemist as the matrices are very complex and present many background interferences. The Stockholm Convention [17] has identified a list of compounds or classes of compounds as persistent organic pollutants based on their persistence, toxicity and tendency to bioaccumulate and biomagnify in adipose tissue. The initial list included 12 persistent organic pollutants (POPs), whereas additional 16 POPs were included in the list by 2017.

Aquatic organisms are exposed daily not only to the known legacy contaminants, but also to their degradation products and/or other chemicals currently in use but not monitored. A survey of the analytical methods for monitoring chemical pollutants in surface waters in Europe showed 
that approximately $75 \%$ of the 41 hazardous chemicals proposed for monitoring under the European Water Framework Directive were reliably monitored. Some substances, such as shortchain chlorinated paraffins (SCCPs), polybrominated diphenyl ethers (PBDEs), tributyltin compounds, certain organochlorine pesticides, and six-ring PAHs, are difficult to monitor due to the lack of validated analytical methods or insufficient sensitivity [18]. Beside the target compound analysis, a challenge in the environmental testing is the identification of other nontargeted compounds present in the samples that might be environmentally significant.

While the requirements for environmental testing continue to grow ever more challenging, the introduction of comprehensive two-dimensional gas chromatography $(\mathrm{GC} \times \mathrm{GC})$ provided a welcome relief owing to the significant increase in the separating power, peak capacity and speed of analysis offered by the technique. This review focuses on representative applications of GC $\times$ GC in the environmental field published in the last decade and provides a brief overview of the different methodologies used for both quantitative and qualitative analyzes.

Comprehensive two-dimensional gas chromatography hyphenated to universal or selective detectors proved to have an outstanding potential for the development of multi-residue methods [19]. GC $\times$ GC was successfully used to accurately quantify target compounds in different environmental matrices. Target compound analyses require method optimization and quality assurance/quality control procedures to be implemented to assure that the compounds are quantitatively extracted from the matrix, the background interferences can be effectively separated from the targets, and the data produced are accurate. Using a technique as powerful as GC $\times \mathrm{GC}$ has proved to be of significant benefit owing to both its quantitation and screening capabilities. 


\subsubsection{Water, wastewater, leachate}

Water contamination comes from many sources, such as industrial and agricultural activities, urban wastewater emissions, and accidental spills. The deterioration of the aquatic environment poses toxicological risk for human health. Protecting the aquatic environment became the mandate of many regulatory bodies, and, as a result, many compounds are now regulated [20] or are proposed as an addition to the priority substances list [17,21] Because the number of chemicals currently produced and used is much higher than the regulated compounds list, there is always the need to identify potential new contaminants present in the environment that might have biological effects. Monitoring these hazardous chemicals in water is difficult as the samples are very complex, and no validated, sufficiently sensitive methods that are applicable under routine laboratory conditions are often available [18]. Recent applications of GC $\times \mathrm{GC}$ are summarized in AppendixA, Table A.1 and further discussed below to emphasize the importance of using this type of technique.

\subsubsection{Hydrocarbons / Polycyclic Aromatic Hydrocarbons (PAHs)/PAH Derivatives}

Frequently found in water samples, PAHs are hazardous contaminants known to bioaccumulate and to have toxic effects on animals and humans. There is a continuous need of developing analytical methods to allow their fast and reliable detection with better sensitivity and resolution in complex matrices. One of the first papers reporting the use of GC $\times \mathrm{GC}$ for the analysis of PAHs and derivatives (oxy-PAHs, nitro-PAHs, methyl-PAHs) in water samples was published by Tobiszewski et al. [22]. The water samples were collected from different sources: Baltic Sea, local rivers and lakes, and drinking water. An optimized GC $\times$ GC -TOFMS method was able to separate 43 target compounds in the first dimension in under 30 minutes. SulejSuchomska et al. [23] developed an SPME-GC $\times$ GC-TOFMS method for the simultaneous 
analysis of 16 PAHs in airport runoff water samples. After SPME optimization, the method was further validated and applied to water samples collected from different international airports. The results showed that regardless of the airport, chrysene, phenanthrene and pyrene were the most abundant PAH compounds detected in all the analyzed samples. The authors concluded that, in addition to separating and quantitating the target compounds, the use of GC $\times \mathrm{GC}$ could provide more information on the content of different groups of xenobiotics in runoff waters.

To simultaneously visualize all bioremediation fractions, GC $\times \mathrm{GC}$ coupled with quadrupole mass spectrometry was used by Beškoski et al. [24] in a recent biodegradation study of isoprenoids, steranes, terpanes, phenanthrenes and methyl-phenantrenes. Terpanes, important biomarkers in petroleum, co-elute in $1 \mathrm{D}$ GC with $\mathrm{C}_{28}-\mathrm{C}_{30}$ n-alkanes, steranes with $\mathrm{C}_{26}-\mathrm{C}_{29} \mathrm{n}$ alkanes, and phenanthrenes with $\mathrm{C}_{17}-\mathrm{C}_{20} \mathrm{n}$-alkanes, but they were all separated in the second GC $\times$ GC dimension. Fifty six compounds were identified in the samples, including n-alkanes and isoprenoid aliphatic alkanes (m/z 71), terpanes (m/z 191), steranes (m/z 217), and phenanthrenes $(\mathrm{m} / \mathrm{z} 178,192,206,220)$. The changes in distribution and abundance of the target compounds were monitored using GCxGC and GC, which helped evaluate the efficiency of the bioremediation processes.

\subsubsection{Organohalogens and Pesticides}

The detection of pesticides in aqueous samples, particularly in drinking water samples where concentration limits are set by regulations, is very important, as many of them are persistent hazardous contaminants. Nine organochlorine pesticides (OCPs) were included in the initial list of 12 persistent organic pollutants (POPs), and an additional 7 OCPs were included in the POP list by 2017. Highly selective and sensitive methods for the analysis of organohalogens and 
pesticides based on a variety of LC or GC approaches have been described in the literature throughout the years. The need for improved resolution and accuracy, as well as low-level detection for pesticide analysis, were the focus of many research groups using $\mathrm{GC} \times \mathrm{GC}$ [4], and the number of published applications using $\mathrm{GC} \times \mathrm{GC}$ has increased substantially in the last decade. GC $\times$ GC was coupled with high resolution TOF MS (HRTOFMS) for the determination of ultra-trace concentrations of 23 OCPs in river water samples [25]. The samples were extracted by an optimized SBSE method followed by thermal desorption directly to GC×GC without any additional clean-up steps. Several compounds partially or completely co-eluting in the first dimension because of a short, narrow-bore DB-5 column used, were separated in the second dimension or were spectrally deconvoluted. Two-dimensional gas chromatography with very narrow mass windows (e.g. $0.05 \mathrm{Da}$ ) provided additional selectivity for more accurate detection. This approach helped with compound class visualization and identification of minor compounds in the complex environmental samples (Figure 1.3). Using library search (NIST and in-house pesticide library), 2D gas chromatography with $0.05 \mathrm{Da}$ wide window, and formula calculation using accurate mass molecular ion, the authors identified 20 non-target compounds: 8 pesticides and 1 pesticide degradation product (molinate, diazinon, bromobutide, simetryn, dimethametryn, pretilachlor, thenylchlor, pyributicarb, and pentachloroanisole), 6 PAHs (phenanthrene, anthracene, fluoranthene, pyrene, four-ring PAHs isomer, five-ring PAHs isomer), 3 PCBs (dichloro-, tetrachloro-, and hexachlorobiphenyl isomers), and 1 pharmaceutical/personal care product and its metabolite (triclosan and triclosan-methyl). 

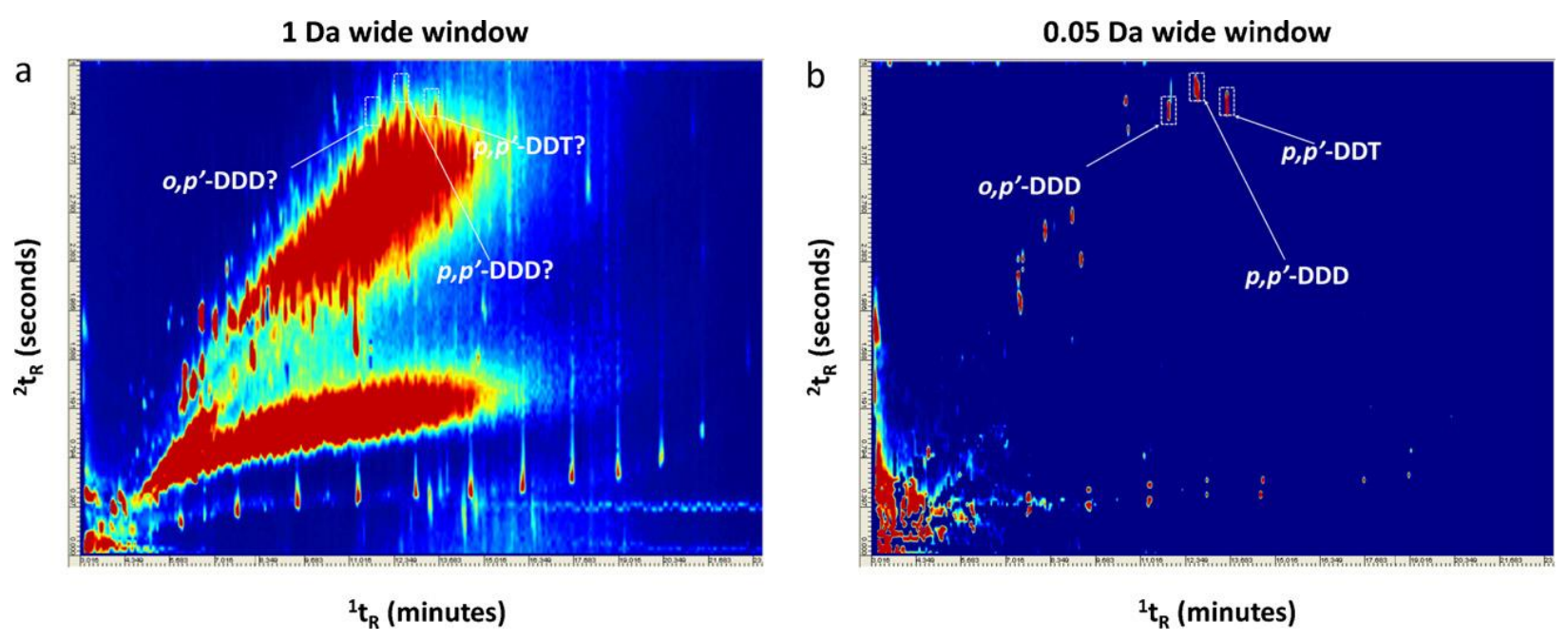

Figure 1.3 Comparison of o,p_-/p,p_-DDD and p,p_-DDT separation using two-dimensional extracted ion chromatogram (m/z 235.0083) obtained by SBSE-TD-GC $\times$ GC-HRTOFMS of river water. (a) 1.0 Da wide window and (b) 0.05 Da wide window. Reprinted from Ref. [25], Copyright (2011), with permission from Elsevier.

Quadrupole MS detector was also evaluated for pesticide analysis in drinking water by Purcaro et al. [26]. The extraction of 28 target pesticides was performed using direct immersion SPME. For this study, SLB-5ms coupled with the ionic liquid SLB-IL59 was the column combination of choice. The rapid scanning MS was operated in full-scan mode with a scan speed of $20000 \mathrm{amu} / \mathrm{s}$ and mass range of m/z 50-450. A minimum of 10 data points/peak were necessary for peak reconstruction and quantification purposes, and this was achieved for peaks with widths > $300 \mathrm{~ms}$. TOFMS is a more suitable detector for the narrow second dimension peaks generated; however, one of the advantages of using qMS was the selected ion monitoring operation option, resulting in enhanced sensitivity.

$\mathrm{GC} \times \mathrm{GC}-\mu \mathrm{ECD}$ and $\mathrm{GC} \times \mathrm{GC}-\mathrm{TOFMS}$ are used routinely for the analysis of PCBs, organochlorine pesticides and chlorobenzenes in water samples and passive samplers [27]. The 
method was initially developed and accredited for solid samples; details regarding the GC×GC separation are presented in this review in Section 1.2.3.2 [28]. Beside the target analysis, this method is also used to screen for the presence of other halogenated compounds. GC $\times$ GC-TOFMS is used as complementary analysis to confirm unusual concentration levels or patterns in the samples, as well as to identify and confirm the non-targeted contaminants when observed in the 2D chromatograms.

\subsubsection{Other compounds}

Benzothiazoles, benzotriazoles and benzosulfonamides, high-production chemicals, were reported for the first time in aqueous samples by using GC $\times \mathrm{GC}-\mathrm{TOF}-\mathrm{MS}$ [29]. As the authors indicated, it is environmentally significant to monitor these compounds as they are not fully removed in wastewater treatment plants and benzotriazoles are classified as emerging pollutants. Three different column set-ups were tested to optimize the $\mathrm{GC} \times \mathrm{GC}$ separation of the target compounds, with the authors concluding that the best separation was achieved when using TRB$5 \times$ TRB-50HT column combination. To emphasize the advantages of $\mathrm{GC} \times \mathrm{GC}$, GC-MS analysis was also performed and compared. The enhanced chromatographic resolution enabled the authors to separate the target analytes, identify minor compounds that would be missed with a 1D-GC technique, and to improve data accuracy (overestimation was eliminated). For the first time, two of the benzotriazoles that co-elute in LC analysis were resolved and reported for both QC and real water samples. In another multiresidue analysis study, the authors showed that a technique such as $\mathrm{GC} \times \mathrm{GC}$ was essential for the analysis of benzothiazole and benzotriazoles at low levels [30]. 
The excellent performance of GCXGC-TOFMS in the determination of emerging contaminants of concern in wastewater and soil samples was demonstrated by Prebihalo et al. [31]. To process the data and minimize this time consuming step, the authors used a targetdiscovery method based on GC×GC-TOFMS deconvolution combined with the reference method function within the ChromaTOF software (LECO Corp.). Using this approach with further confirmation by analyzing standards, halogenated benzotriazoles were identified at high concentrations in some influent and effluent water samples. These compounds were initially misidentified as isocyanates by library searching. The mass spectral data was similar between the standards analyzed and samples, but the first and second dimension retention times were very different than those of the standards confirming the misidentification. Similarly, halogenated benzotriazoles as well as their degradation product phenol were identified at lower levels in the soil samples. The authors stressed the importance of this targeted-discovery approach for resolving complex matrices, identifying emerging pollutants and their degradation products, as well as helping with the preliminary investigation of environmental fate.

Nonylphenols and their derivatives, used in the production of plastics and surfactants, are chemicals of concern for humans and wildlife due to their estrogenic effects. They were detected in many environmental matrices. The potential of GC×GC-TOFMS was demonstrated for the specific determination of 4-nonylphenol isomers in water and landfill leachate samples [32-33]. Quantitative data were presented to show significant differences among technical compositions highlighting the number of peaks detected and the corresponding \% peak areas. In a 2009 study [23], 153 to 204 total nonylphenol isomers were detected, depending of the technical mix source. The enhanced resolution of $\mathrm{GC} \times \mathrm{GC}$ allowed the separation and identification of all major 4nonylphenol isomers, in addition to the detection of four isomers not reported before (Figure 1.4). 
As the authors emphasized, using a technique such as $\mathrm{GC} \times \mathrm{GC}$ may be essential in the detection of minor constituents of the technical mixes. The environmental impact of the study might be significant as the "unidentified" isomers were found to be persistent, and their estrogenic effects are not known.

To meet the requirements of the European Union directives (LOQ $60 \mathrm{pg} / \mathrm{L}$ ), a twodimensional heart-cut GC-MS/MS method for the quantification of tributyltin (TBT) in surface waters was developed by Devos at al. [34]. Even though it is not a truly comprehensive GC application, this method was significant as it highlighted the importance of getting a better chromatographic separation from both matrix and/or reagent impurity interferences in order to meet the more stringent requirements for environmental testing. SBSE combined with in-situ derivatization was applied for sample preparation followed by GC-MS/MS and heart-cut GCMS/MS analyzes. GC-GC-MS/MS set-up consisted of a Deans switch, FID to monitor the effluent of the first column to select the heart-cut window, and an HP-5MS/DB-17MS column combination. TBT was clearly separated from interferences, and additional transitions could be used for confirmation. Linearity in the range of $0.05-4 \mathrm{ng} / \mathrm{L}$ was achieved with this set-up. 


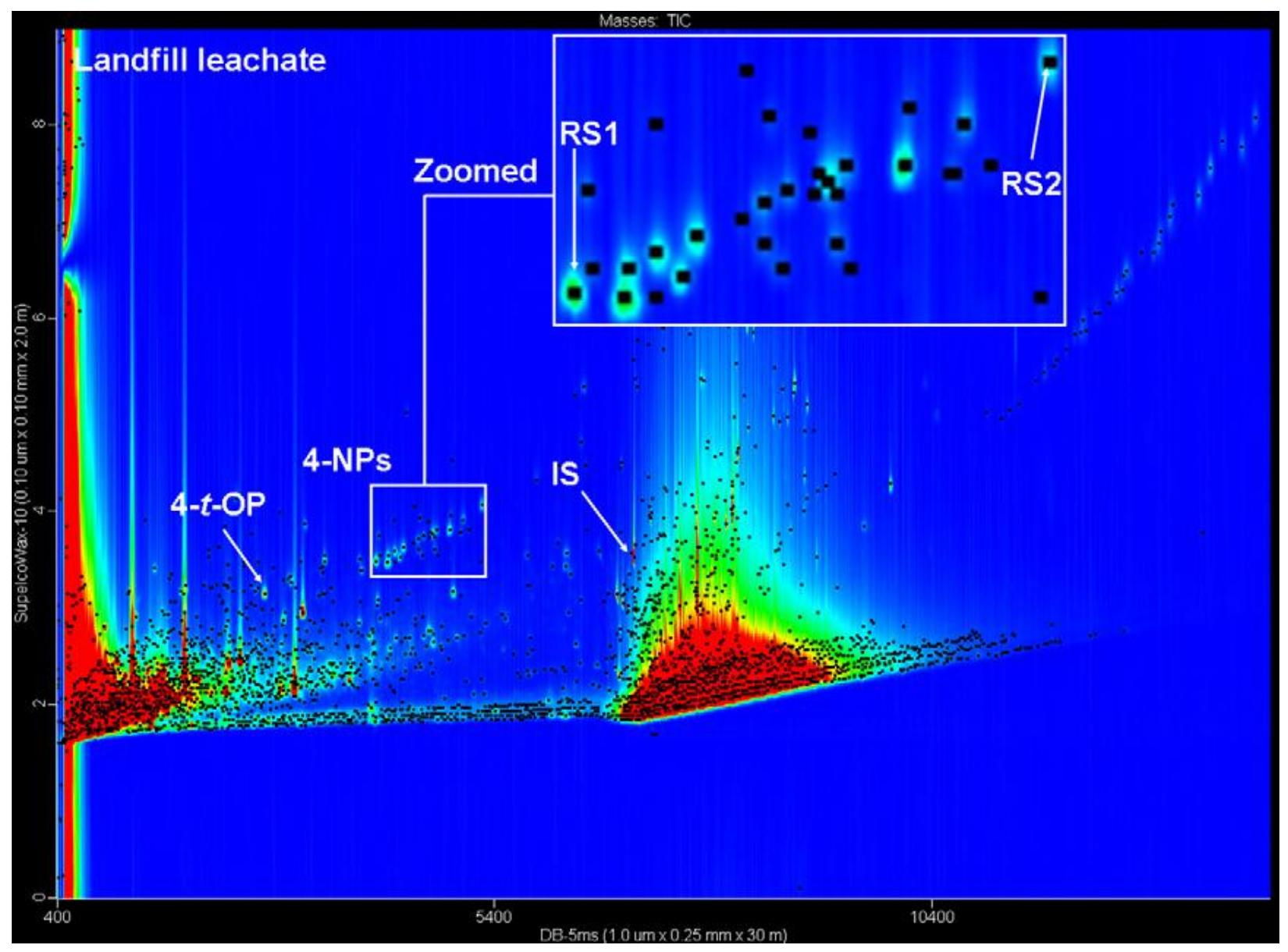

Figure $1.4{ }^{2} \mathrm{D}$ contour plot of landfill leachate analyzed by SDE-GC $\times$ GC/TOFMS. Reprinted from Ref. [33], Copyright (2012), with permission from Elsevier.

Lima Gomes et al. [35] used SPME-GC×GC-TOFMS with an Rtx-5MS x Rtx-200 column set to separate and quantify emerging contaminants in river water samples, including 11 steroids, caffeine and methylparaben. The analyses were performed without any derivatization prior to injection; no significant degradation in chromatographic or instrument performance was observed. This method permitted a complete separation of structurally similar analytes from each other and from interferences without co-elutions, and was further applied to the analysis of spiked water samples. 
Synthetic musks isolated by SPME from various water samples (river, wastewater and groundwater) were analyzed by GC×GC-TOFMS [36]. Fourteen target compounds were separated on SLB-5MS $\times$ BPX-50 column set and quantified by using the standard addition method. The method's LODs ranged from 0.05 to $2.95 \mathrm{ng} / \mathrm{L}$, while LOQs ranged from 0.1 to 9.84 ng/L with HSA (3-Phenylpropan-1-ol) being the poorest performer. Except for three target analytes that were not present in any of the samples, all the other targets were detected. Depending on the sample type, their concentrations ranged from not detected or very low levels in clean water samples to higher concentrations in wastewaters (e.g. galaxolide ranged from 0.09 to 1.13 $\mu \mathrm{g} / \mathrm{L}$ ). Using statistical tools, the authors used the data to assess the similarities between the different water sources. Finding these compounds in water sources used for drinking water was important, the authors concluding that the bioaccumulation factors should not be neglected even though these compounds were present at low concentrations.

Li et al. [37] developed a screening method for disinfection by-products (DBPs) in drinking water samples. The samples were extracted by SPE and analyzed by GC×GC-qMS. A rapid scanning GC×GC-qMS system and OECD QSAR Toolbox (http://www.qsartoolbox.org) were combined for identifying and prioritizing volatile and semi-volatile DBPs in drinking water. By using this approach, over 500 compounds were tentatively identified. A total of 170 DBPs representing 14 chemical classes were identified in different water samples, of which 58 were found to be actually or potentially genotoxic. Selected 48 compounds were further confirmed by analyzing standards. The authors identified several non-halogenated amides and halogencontaining DBPs that were not reported in a previous study and provided a list of all the 170 identified DBPs. This study illustrated the importance of non-targeted analysis for prioritizing which pollutants should be further monitored in drinking water. 
A non-targeted GC $\times$ GC-TOFMS method for the analysis of compounds with steroid structure was developed by Kopperi et al. [38]. This method combined with statistical data interpretation was used to assess the purification efficiencies and to identify possible transformation products of the parent compounds in the suspended solids, effluent water and sludge collected from 10 wastewater treatment plants. The data processing was initially done by an automated identification by ChromaTOF software, followed by retention indices assignment, alignment using metabolomics data analysis software, and mass spectral match. A chemometric model was applied to quantify tentatively identified four-ring steroids; concentrations could be predicted based on mass spectrum and detector response without prior knowledge of compound identity. The major classes of steroids detected in the study were androstanes, estranes, cholestanes and pregnanes. The authors found that most of the steroids were removed from the aqueous phase during the treatment. Other emerging micropollutants were identified in the water samples, ibuprofen, caffeine, carbamazepine and cotinine being the most abundant. The advantages of GC $\times$ GC-TOFMS analysis were pointed out, including high peak capacity and the capability to generate analytical profiles as a means for screening for variations between different samples and avoiding the time-consuming process of identification and quantitation of individual compounds.

Guo et al. [39] combined the data from GC $\times$ GC-TOFMS and gas chromatographyolfactometry mass spectrometry (GC-O/MS) along with retention indices to identify odorants in river water samples. The liquid-liquid extracts of blanks and samples were analyzed by both techniques. Four typical odorants were added to the $\mathrm{C}_{7}-\mathrm{C}_{30}$ alkane solution when calculating the retention indices to verify the correspondence between the olfactometry peaks and the corresponding compound information from GC $\times \mathrm{GC}$. Matching the peaks obtained by the two techniques allowed neglecting the compounds without odor; thus, fewer standards were required 
for further confirmations. GC-O/MS identified thirteen olfactometric peaks; only two were tentatively identified by the NIST library. When GC×GC was employed, over thirty potential odorants corresponding to the thirteen olfactometry peaks were tentatively identified by the NIST library. Even though an olfactometry peak was not detected due to solvent delay, GC×GC detected another septic odorant, dimethyl disulfide (a total of 14 odorants were confirmed). The advantages of combining the data obtained from the $\mathrm{GC} \times \mathrm{GC}$ system included the assignment of potential odorants to all 13 olfactometry peaks, as well as tentatively identifying other odorants present in the water samples.

\subsubsection{Multiresidue Methodologies}

With over 100,000 chemicals used daily, the need for multiresidue methods to prioritize the monitoring of potentially persistent, bioaccumulating and toxic contaminants is increasing. Recently, several water analysis studies implemented this approach. Due to their high mass discharge into the aquatic environment, pharmaceuticals and personal care products (PPCP) are considered emerging contaminants of concern and their monitoring is considered necessary. PPCPs include thousands of distinct chemicals continuously used in a broad range of human and veterinary therapeutic activities, as well as other consumer products. A variety of multiresidue analytical methodologies (LC/MS or GC/MS) including either priority or emerging pollutants as target analytes in aqueous matrices do not permit their simultaneous determination in one analytical run. Several GC $\times$ GC-TOFMS methods for multiresidue analysis of priority pollutants, pharmaceuticals and other emerging contaminants in aqueous matrices have been reported in the recent years [30,40]. Matamoros et al. [30] developed a method targeting 97 organic contaminants at trace level in river water collected from four different rivers. The analytes included 13 
pharmaceuticals, 18 plasticizers, 8 personal care products, 9 acid herbicides, 8 triazines, 10 organophosphorus compounds, 5 phenylureas, 12 organochlorine biocides, 9 polycyclic aromatic hydrocarbons (PAHs), and 5 benzothiazoles/benzotriazoles. The contour plots for both quality control and real river samples revealed many ${ }^{1} \mathrm{D}$ GC coelutions that were resolved by using GC $\times$ GC. This enhanced separation resulted in elimination of analyte overestimation and provided more accurate data.

Thirteen personal care products (PCPs), 15 PAHs and 27 pesticides [40] were separated by using the Rxi-5 x Rsi-17 and Rxi-5 x LC-50 column combinations, the former combination being the choice for quantitative analysis. The detection limits achieved for this method were lower than in a similar study reported in 2010 [30]. Based on analyte recoveries obtained from Mili-Q, river and waste waters, the authors discussed the SBSE lower recoveries for the more hydrophobic compounds, as well as the signal enhancement in TOFMS, concluding that both were observed due to the matrix complexity. Different approaches to compensate for this were suggested: surrogate addition from the beginning of the sample processing, standard addition or sample dilution. To further emphasize the advantage of using this technique beside the quantitation of target compounds, the authors used the features of ChromaTOF software (e.g. bubble plots) and proposed methods to fingerprint the water sample contaminants, compare the sources of contamination along the river and monitor variations over time. Non-target compounds such as 4-chlorophenol and loratadine were identified and were proposed to be added in the future for quantitation. Finally, the data obtained for non-target compounds helped to rapidly select chemical markers of water contamination.

The benefits of using $\mathrm{GC} \times \mathrm{GC}$ and structure-activity modeling for screening and preliminary risk assessment of organic contaminants in surface water, soil and sediment collected 
from a known contaminated industrial site were presented by Bastos and Haglund [41]. The enhanced resolution of $\mathrm{GC} \times \mathrm{GC}$ offered clear advantages over ${ }^{1} \mathrm{D}-\mathrm{GC}$ : less-purified extracts could be used, and less-abundant contaminants could be separated from bulk contaminants or matrix interferences. However, while GC×GC-TOFMS provided enhanced resolution, it also produced larger datasets that were more time consuming to evaluate due to the overwhelming number of peaks. By using different standard mixes, between 100 and 500 compounds were structurally identified and quantified or semi-quantified in each sample. The most abundant compounds found in sediment samples were phthalates and alkanes; in water samples they were phthalates and their degradation products, while in soil samples a variety of contaminants were found: PAHs, chlorinated compounds, phthalates, and several biotransformation products of PAHs and alkanes. Alkyl-substituted PAHs were more abundant in terms of presence and concentration in these samples than in the sediment samples. Based on the data obtained, the authors were able to predict the toxicity of the compounds found by using the ECOSAR software and provide an initial assessment of the potential environmental impact.

Wooding et al. [42] reported a simplified method for the detection and quantification of endocrine disrupting chemicals (EDC), emerging EDCs and antiretroviral drugs. Disposable PDMS samplers were developed in-house for the extraction of surface waters, followed by thermal desorption $\mathrm{GC} \times \mathrm{GC}-\mathrm{TOFMS}$ The criteria used for identification were mass spectral match $\geq 80 \%$ and retention time matching set to $1 \mathrm{~s}$ for ${ }^{1} \mathrm{D}$ and $0.1 \mathrm{~s}$ for ${ }^{2} \mathrm{D}$. In addition, retention indices were calculated for non-target compounds, and UHPLC-MS/MS was used as a complementary technique. The untargeted screening revealed the presence of over 3000 compounds owing to the increased resolving power and sensitivity of GC×GC-TOFMS. They included PPCPs, sunscreen ingredients, pesticides, hormones and fragrances. The untargeted 
screening results were used to select 12 compounds for target analysis, with the 10 analyzed by GC $\times \mathrm{GC}$ representing different classes of compounds (pesticides, plasticizers, fragrance, PPCPs). Sufficient separation was achieved between two chlorinated pesticides, chlorpyrifos and metolachlor, to enable quantification (see Figure 1.5). Surface water samples collected from urban areas showed a higher presence of pesticides and PCPs. Despite its current use, DDT was not detected. The authors suspected that the confirmed estrogenic activity in drinking water was due to a combined effect of more than one chemical, suggesting that the monitoring would require complementary detection techniques. The detection of efavirenz $(\mathrm{GC} \times \mathrm{GC})$ and nevirapine (UPLC), both antiretroviral drugs, was of concern.

Blum et al. [43] reported a screening approach to prioritize potentially persistent, bioaccumulating and toxic wastewater contaminants. Their strategy consisted of two stages: first, non-targeted analysis was used to detect the analytes, approximately 300 which were tentatively identified. In the next stage, selected environmentally relevant contaminants were analyzed by target approaches. By using this approach, 26 compounds from different classes (including biocides, fragrances, food additives, UV stabilizers, plasticizers, organophosphorus flame retardants, etc.) were further considered for targeted analysis and removal efficiency studies. Based on this study, the authors reported for the first time compounds found in the on-site sewage treatment facilities effluents that could potentially impact groundwater. 


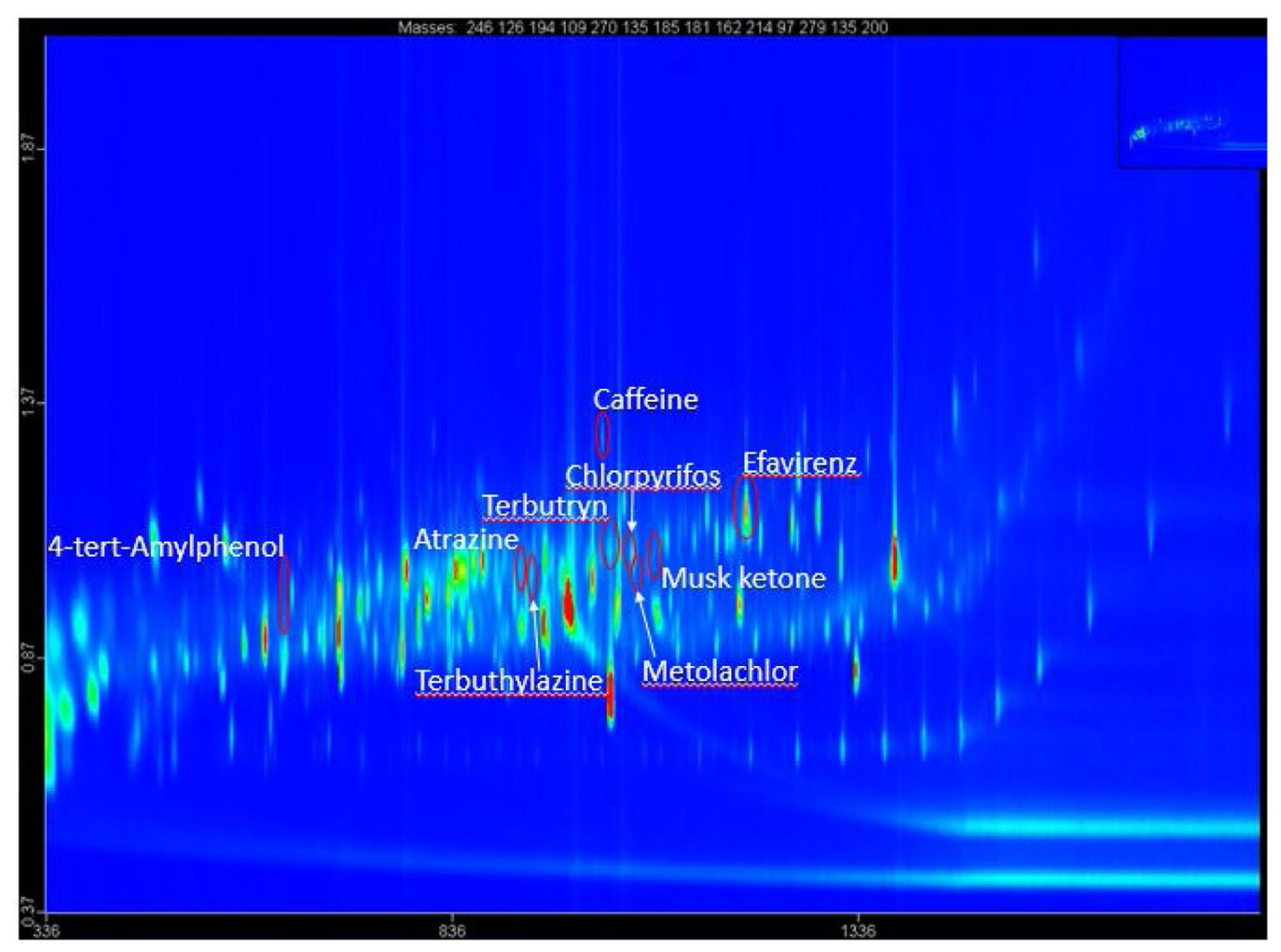

Figure 1.5 Contour plot of a reconstructed ion chromatogram (RIC) (GC×GC-TOFMS) of surface water collected at site 2 (Otter Bridge) in Rietvlei Nature Reserve. Target analytes detected are indicated. RIC: 246, 126, 194, 109, 270, 135, 181, 185, 162, 214, 97, 279 and 200 m/z. Reprinted from Ref. [42], Copyright (2017), with permission from Elsevier.

Wanda et al. [44] combined the resolving power and sensitivity of GC×GC-HRTOFMS to develop a multiresidue method for the analysis of different emerging micropollutants (carbamazepine, galaxolide, caffeine, tonalide, 4-nonylphenol, and bisphenol A) in various water matrices. The clear advantages of using the GC×GC-HRTOFMS system (mass accuracy $<1$ ppm), including structured chromatograms, increased sensitivity and peak capacity, were highlighted in this study when the detection and separation of 4-nonylphenol was possible. All the analytes were detected in the water samples tested, bisphenol A being identified as the most 
widely distributed emerging contaminant. Further, the authors statistically evaluated the temporal and spatial variations based on the data obtained, concluding that the identified targets posed ecotoxicological risks to aquatic life as well as communities.

\subsubsection{Biota}

Analysis of biota samples poses challenges for both sample preparation and instrumental analysis, as the contaminants tend to bioaccumulate and bio-magnify in adipose tissue. The matrix complexity requires powerful separation techniques to separate the target compounds from the matrix interferences. The number of papers showing the use of $\mathrm{GC} \times \mathrm{GC}$ for the analysis of biota samples has increased substantially in the last decade; a summary of the recent applications is presented in Appendix A, Table A.2.

PCBs, a mix of 209 congeners, tend to bioaccumulate and biomagnify in the environment and have toxic effects to wildlife and humans. Their accurate determination requires methods capable of separating them in complex matrices, as well as separating the more toxic congeners [45] from the more abundant ones with less toxic effects. Taking advantage of the increased peak capacity and resolving power of $\mathrm{GC} \times \mathrm{GC}$, enhanced separation of $\mathrm{PCB}$ congeners was achieved by using a non-polarxionic liquid column set. A total of 196 out of the 209 PCB congeners were resolved. All PCB congeners in Aroclor 1242 (except for PCB12 + PCB13) and in Aroclor 1260 were separated on this column set (SPB-Octyl×SLB-IL59) [46]. Megson et al. [47] separated 188 of the $209 \mathrm{PCB}$ congeners in the second dimension using Rtx-PCB $\times \mathrm{Rxi}-17$ column set. The group further identified 12 more congeners by manual data processing and suggested caution when quantitating homologues from different series that overlap. Aroclor analysis showed that all congeners present in Aroclors 1254 and 1260 were separated, 113 of the 115 congeners in Aroclor 
1248 (co-elution of PCB88 + PCB95), 96 of the 99 congeners in Aroclor 1242 (co-elution of PCB20 + PCB21 + PCB33) and 63 of the 66 congeners in Aroclor 1016 (co-elution of PCB20 + PCB21 + PCB33). The method was applied to extracted whiting and guillemot liver samples, the authors highlighting its potential for PCB fingerprinting in environmental forensic studies.

The feasibility of $\mathrm{GC} \times \mathrm{GC}$ for group type separation among different classes of POPs was assessed by different groups in an attempt to achieve a complete separation [48-49]. With the goal of finding the best column set for a fast screening method of eight POP classes, Bordajandi et al. tested different column sets [49]. Even though a complete separation was not achieved, some of the column sets tested presented particular benefits, improving within-class separation and isolating selected families from the rest of the POPs. The method was applied to polar bear fat samples where toxaphene bands were identified and completely isolated from the rest of POPs by using BP-10×BP-50 column set.

Kalachova et al. [50] developed and validated a GC×GC-TOF-MS method for analyzing 18 PCBs, 7 PBDEs, and 16 PAHs in fish samples. Separation of all target analytes (see Figure 1.6) was observed on a BPX $5 \times \mathrm{BPX} 50$ column set (e.g. critical groups of PAHs benz[a]anthracene/cyclopenta[cd]pyrene / chrysene, benzo[b]fluoranthene / benzo[j]fluoranthene / benzo[k] fluoranthene, and dibenz[ah]anthracene / indeno[1,2,3-cd]pyrene / benzo[ghi]perylene were resolved). By using large volume injection, the group was able to achieve limits of quantitation that met the European standards. 


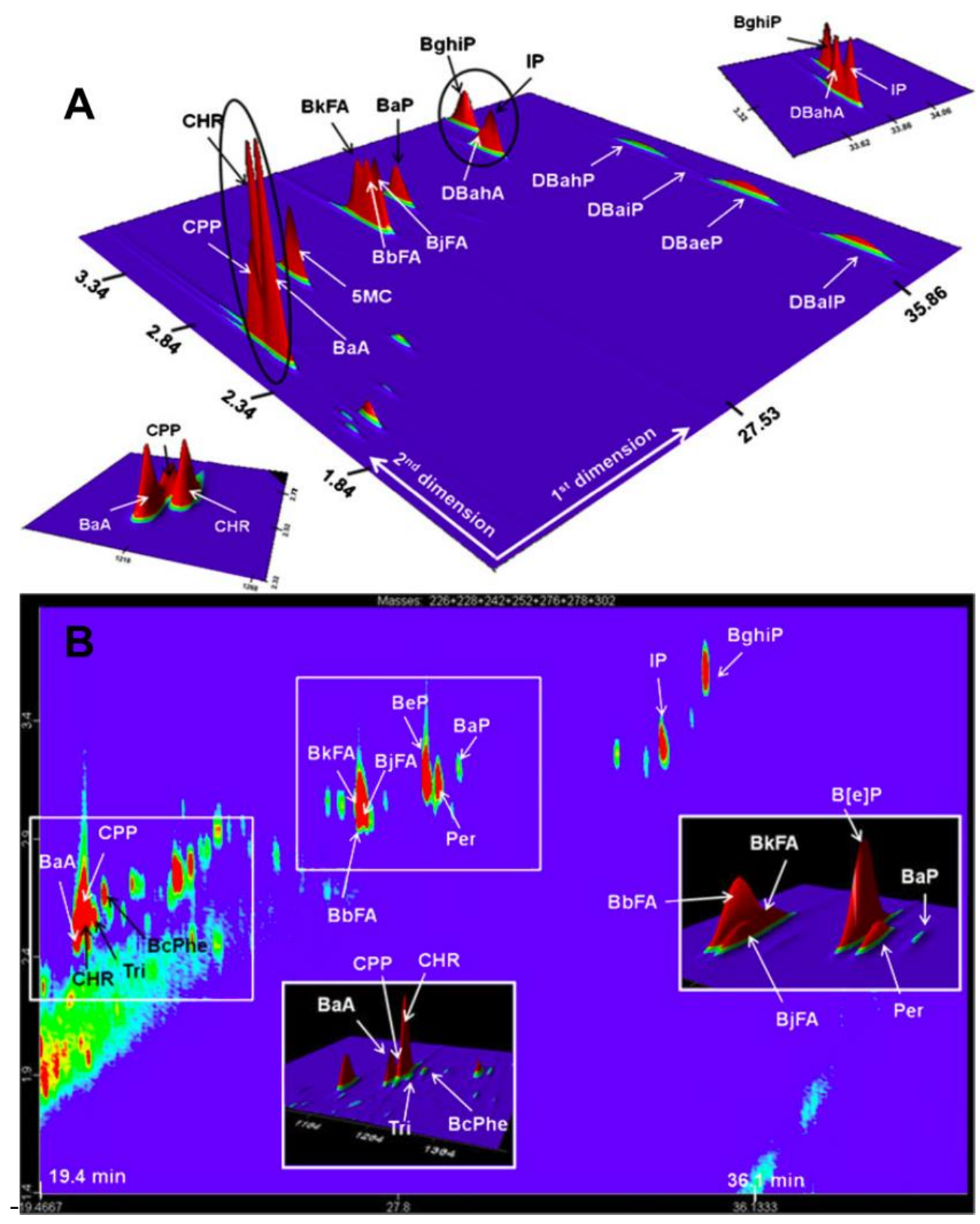

Figure 1.6 Example of a GC×GC-TOFMS chromatogram of PAHs separated on BPX5×BPX50 column system. Sum of $\mathrm{m} / \mathrm{z}$ 226, 228, 242, 252, 276, 278, and 302 is displayed. A). Spiked fish muscle tissue (concentration $100 \mathrm{ng} / \mathrm{mL}$, corresponding to $10 \mu \mathrm{g} / \mathrm{kg}$ fish muscle tissue); B). SRM 1974b Mussel Tissue. Adapted by permission from Springer Nature: Ref. [50], Copyright (2012). 
Standard methods for identifying the components of unresolved complex mixtures (UCM) in environmental samples were labor intensive, often resulting in poor chromatographic separation and identification of the target compounds. GC $\times$ GC-TOFMS was used for tentative identification of petroleum-derived hydrocarbons in UCM in mussel samples by Booth et al. [51]. The UCM contained thousands of largely unidentified branched alkyl homologues of known aromatic hydrocarbons, such as branched alkyl benzenes, branched alkyl tetralins, and branched alkyl indanes and indenes, which exhibit very similar MS fragmentation. The combination of 2D separation and data processing algorithms revealed over 3000 individual peaks, some of them being confirmed by this study as being branched alkyl tetralins and branched alkyl indanes.

In addition to monitoring environmental samples for known POPs, there has been a growing interest in the application of $\mathrm{GC} \times \mathrm{GC}$ for the determination of other halogenated contaminants in biota samples [52-58]. Hoh et al. analyzed POPs in fish oil samples using both targeted and nontargeted approaches using direct sample introduction (DSI-GC×GC-TOF-MS) [52-53]. This combined approach increased the scope of organic contaminants monitored: GPC maximized the number of detected compounds, silica SPE and acidification helped elucidating possible chemical composition or structures of the unknowns, DSI enabled large volume injections for dirtier extracts, and $\mathrm{GC} \times \mathrm{GC}$ provided improved separation, sensitivity and provided full scan mass spectra [52]. Multiple classes of POPs were identified in the dietary fish oil supplement (PCB, OCPs, BDEs and toxaphenes) along with other untargeted chemicals (e.g oxybenzone, a common sunscreen agent, and tentatively identified organohalogens). Interestingly, novel flame retardants were found and reported as laboratory contaminants. A follow-up quantitation method for multiple classes of organic contaminants proved the feasibility of DSI-GC $\times$ GC-TOF-MS to accurately quantitate the target compounds. The detection of POPs and other chemicals in fish oil 
further suggested that conventional treatment processes were ineffective at removal of the heavier organic contaminants [53]. Using the same GC $\times$ GC-TOF-MS system [43], this non-targeted approach was successful in separating and identifying halogenated compounds in dolphin [5455] and seabird samples [57]. For instance, 271 compounds were detected in dolphin samples belonging to 24 classes (e.g. PCBs, pesticides and related compounds, methoxy polychlorinated diphenyl ethers, PCDEs, PBBs, PBDEs and polychlorinated styrenes), 86 compounds being anthropogenic contaminants not routinely analyzed [52]. It should be noted that exclusive reliance on commercially available reference standards and mass spectral libraries for non-targeted analysis resulted in missing a significant fraction of existing compounds; thus, de novo interpretation approach was necessary. The results of the analysis were summarized for future reference in comprehensive PDF mass spectral libraries containing information for the identified and unknown compounds $[52,55,57]$.

Pena-Abaurrea et al. [56,59] reported chlorinated and organobrominated compounds identified in farmed and wild bluefin tuna. Improved separation obtained within and between different classes of organobromines, as well as their separation from other organohalogens and matrix components, enabled micropollutants profiling in the samples. As a consequence of the increased peak capacity, resolution and structured chromatograms, the identification of other brominated compounds was achieved, some of them reported for the first time in this study (e.g. tri- and tetra- brominated hexahydroxanthene derivates and one tetra-dimethoxy-BDE).

In order to maximize compound separation, eliminate matrix interferences and improve the selectivity, HRTOF-MS was used in combination with $\mathrm{GC} \times \mathrm{GC}$ for simultaneous analysis of different classes of compounds [60]. PCB and PCN quantitation, as well as screening for other non-targeted compounds in three fish species were presented by Xia et al. [60]. Complete 
separation was achieved for all the target compounds (18 PCBs, 16 PCNs) and matrices. The results were in good agreement when compared with those obtained by classical GC-HRMS analysis. Taking into account the multiple fractionation steps and analytical runs required for GCHRMS, GC $\times \mathrm{GC}$ was able to accurately quantitate the target compounds and identify other nontargeted compounds in a single analytical run, resulting in significant time savings. The authors indicated that the data files acquired were very large (5-10 GB), and the data processing times and quantitation software were the limiting factors for the method.

\subsubsection{Solid Samples: Soil, Sediment, Sludge}

Analytical chemists face many challenges when trying to separate and accurately quantitate selected target compounds in solid matrices due to matrix complexity. GC $\times \mathrm{GC}$ offers many advantages over conventional 1D-GC methods in the analysis of POPs and emerging contaminants in solids. Multiple applications of $\mathrm{GC} \times \mathrm{GC}$ demonstrated the benefits and its potential implementation as routine analytical method for environmental monitoring. Table A.3 (Appendix A) summarizes more recent applications of $\mathrm{GC} \times \mathrm{GC}$ for soil, sediment and sludge samples analysis.

\subsubsection{PAHs and PAH Derivatives}

A method for the determination of 16 target PAHs in sediments was developed by PenaAbaurrea et al. by using GC×GC-TOFMS [61]. Two column sets were selected for this study, DB-5 $\times \mathrm{BPX}-50$ and $\mathrm{HT}-8 \times \mathrm{BPX}-50$, and different temperature programs and modulation conditions were tested to optimize the separation, minimize peak broadening and avoid the wraparound. The DB-5×BPX-50 set with a 5 s modulation period achieved satisfactory separation of chrysene from benzo[a]anthracene (both m/z 228) and cyclopenta[cd]pyrene (m/z 226), between 
the three benzofluoranthene homologues, and between indeno[1,2,3-cd]pyrene and dibenzo[a,h]pyrene. The method developed allowed a satisfactory separation of difficult pairs/group of PAHs (e.g. benzo[a]anthracene/cyclopenta[cd]pyrene/chrysene) and of other PAH pairs usually indistinguishable even when using MS detection. The authors pointed out that due to improved resolution and identification power of GC $\times$ GC-TOF-MS, the identification of other PAHs separated from the target compounds was possible (e.g. the tentatively identified dibenzo[a,c]anthracene was separated from dibenzo[a,h]anthracene). Along with providing quantitation results, the method also provided $\mathrm{PAH}$ profiles which were very important for tracking down the contamination source.

Chlorinated and brominated PAHs were determined in soil samples by Ieda et al. [62] using GC×GC-HRTOFMS system. Two column sets were tested for the study, BPX-5×BPX-50 and BPX-5 $\times$ LC $-50 \mathrm{HT}$, the first one being selected because of its higher maximum operating temperature $\left(370^{\circ} \mathrm{C}\right)$ and the ordered $2 \mathrm{D}$ chromatograms without wrap-around obtained. The system was tested for linearity, repeatability and LODs by using $19 \mathrm{Cl}-\mathrm{PAH}$ and $11 \mathrm{Br}-\mathrm{PAH}$ standards. The LODs ranged from 0.08 to $0.44 \mathrm{pg}$ for Cl-PAHs and 0.26-3.2 pg for Br-PAHs. The 2D chromatogram of a Soxhlet extracted soil sample analyzed with this method showed the presence of hundreds of Cl-/Br-PAHs, PAHs, PCs, PCBs and PCDFs separated from the UCM, confirming all $19 \mathrm{Cl-PAHs}$ and $3 \mathrm{Br}-\mathrm{PAHs}$ present in the sample. To further identify other chlorinated PAHs present in the soil extract, mass chromatography with $0.05 \mathrm{Da}$ wide $\mathrm{m} / \mathrm{z}$ window was performed for initial identification, while mass chromatography with $0.02 \mathrm{Da}$ wide $\mathrm{m} / \mathrm{z}$ window was performed for Cl-PAHs in order to achieve greater selectivity and more detailed group analysis (Figure 1.7). Using this approach, the authors identified thirty Cl-/Br-PAHs (including 10 higher chlorinated PAHs with penta, hexa and hepta substitution) and ClBr-PAHs, 
with some of these compounds being reported in environmental samples for the first time (e.g. $\mathrm{C}_{14} \mathrm{H}_{3} \mathrm{Cl}_{7}$ and $\mathrm{C}_{16} \mathrm{H}_{3} \mathrm{Cl}_{7}, \mathrm{C}_{14} \mathrm{H}_{7} \mathrm{Cl}_{2} \mathrm{Br}$ and $\mathrm{C}_{16} \mathrm{H}_{8} \mathrm{ClBr}$ ). In addition, 35 other organohalogens were identified in the samples, including PCNs, PCDFs, PCBs, polychlorinated benzonaphthofurans, mixed chlorine and bromine furans, and halogenated organosulfur compound. The acquired full spectral data with exact mass measurements when using GC×GC-HRTOFMS allows post-target analysis at a later date, another great advantage of this technique.
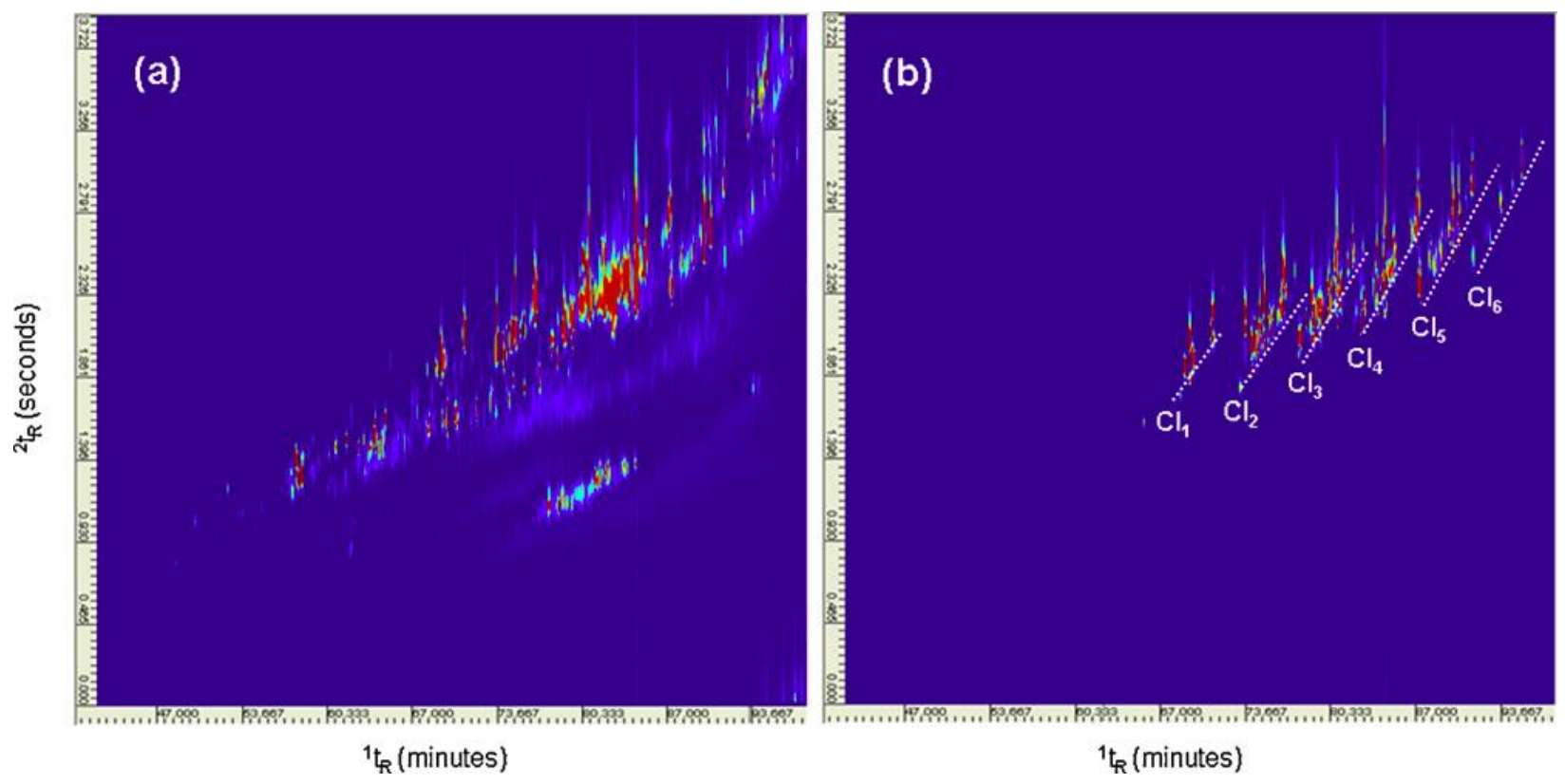

Figure 1.7 Comparison of group type separation using the 2D mass chromatograms obtained using the GC $\times$ GC-HRTOFMS of a soil extract (sum of selected ions for mono to hexa Cl-PAHs; $\mathrm{m} / \mathrm{z}$ 236.0392, 270.0003, 303.9654, 337.9239, 371.8834 and 405.8444). (a) 1.0 Da wide window and (b) 0.02 Da wide window. Reprinted from Ref. [62], Copyright (2011), with permission from Elsevier.

Fernando et al. [63] re-examined a composite soil sample collected after a fire at a plastic recycling facility by using FT-ICR, GC×GC-TOF-MS and GC×GC-HRTOF-MS. The cleaned- 
up ASE extract was analyzed by FT-ICR, then the processed data was further exported to Microsoft Excel where Kendrick mass defect plots using $-\mathrm{H} /+\mathrm{Cl}$ scale were constructed to identify the polychlorinated compounds and visualize the data [64]. Each cluster in the Kendrick plot was separated by $34 \mathrm{Da}$ increments and consisted of isotopic peaks separated by $1.997 \mathrm{Da}$ corresponding to the exact mass difference between ${ }^{35} \mathrm{Cl}$ and ${ }^{37} \mathrm{Cl}$ [64]. This approach led to the identification of halogenated PAHs, e.g. anthracene and/or phenathrene $\mathrm{Cl}_{1}$ to $\mathrm{Cl}_{9}$ substituted congeners, as well as mixed chloro/bromo PAHs (37 compounds detected). Following this tentative identification, the separation of isomers was assessed by using GC $\times$ GC-HRTOF-MS. Ordered structures were observed in the 2D space for structurally related compounds. $\mathrm{Cl}$ and mixed $\mathrm{Br} / \mathrm{Cl}$ congeners appeared as bands of peaks across the two-dimensional plane. Tentative structures were proposed based on exact mass measurements, isotope mass ratios and EI fragmentation patterns (Figure 1.8). The $\mathrm{GC} \times \mathrm{GC}$ chromatographic separation enabled the differentiation of isomeric structures with identical mass spectra. The determination of relative levels of halogenated PAHs was done by GC×GC-TOF-MS, as the GC×GC-HRTOF-MS system was limited by its slow acquisition rate (10 spectra/second) and narrow dynamic range resulting in poorly defined peak shapes. A total of 25 PAHs and halogenated PAHs were identified and quantified using authentic standards. For the halogenated PAHs where standards were not available, the concentration was estimated using the average response factors of structurally related authentic standards. Halogenated PAHs were detected for parent compounds ranging from $\mathrm{m} / \mathrm{z} 128\left(\mathrm{C}_{10} \mathrm{H}_{8}\right)$ to $\mathrm{m} / \mathrm{z} 276\left(\mathrm{C}_{22} \mathrm{H}_{12}\right)$. The highly chlorinated and high molecular weight compounds detected by FT-ICR were not detected by GC×GC-TOF-MS, the authors assuming that they did not elute from the columns used or were below the detection limits. Halogenated congeners of anthracene/phenathrene and fluoranthene/pyrene groups yielded the highest total 
concentrations, while the most abundant mixed halogenated PAH was a monochloromonobromo-fluoranthene/pyrene. This approach led to the identification of approximately 150 formulas corresponding to halogenated and mixed halogenated PAHs, some of them being reported for the first time in environmental samples.

a)

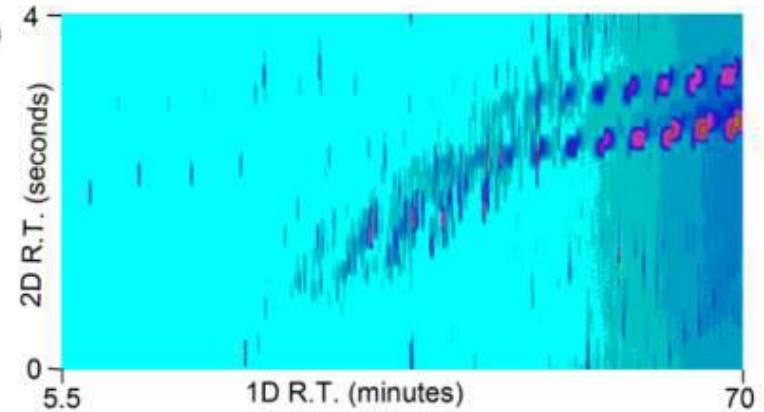

b)

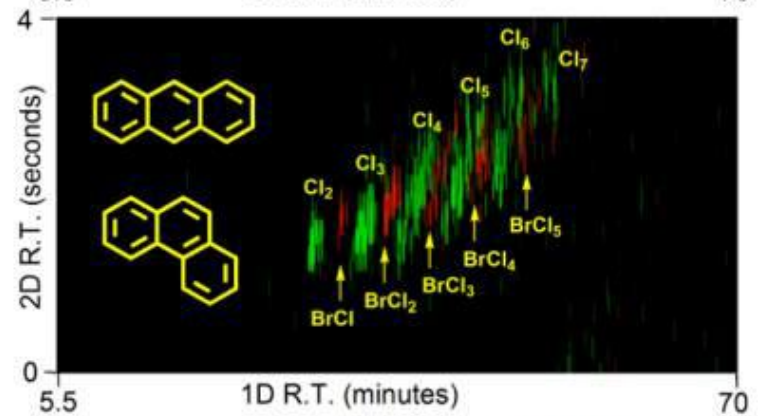

c)
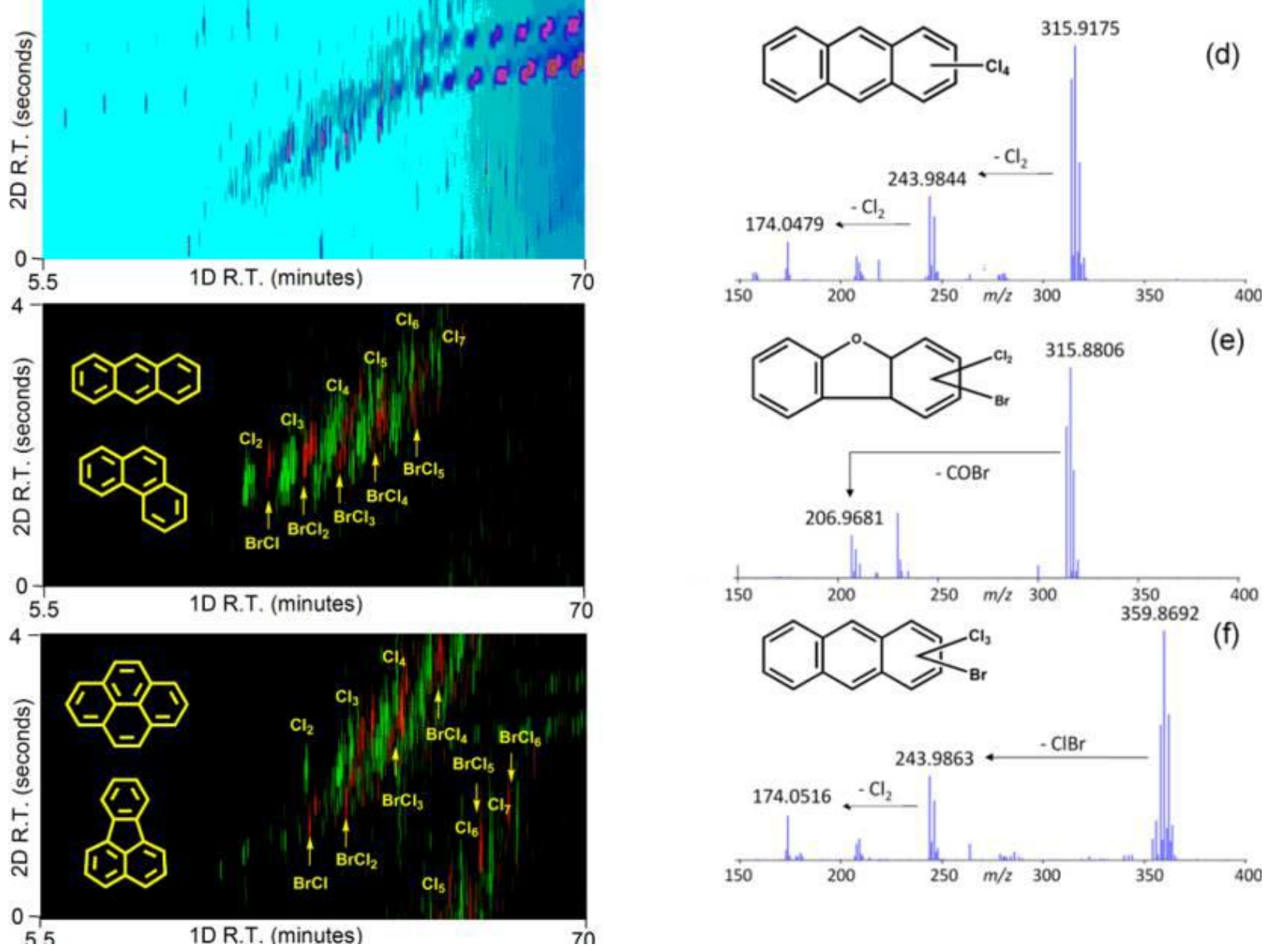

(e)
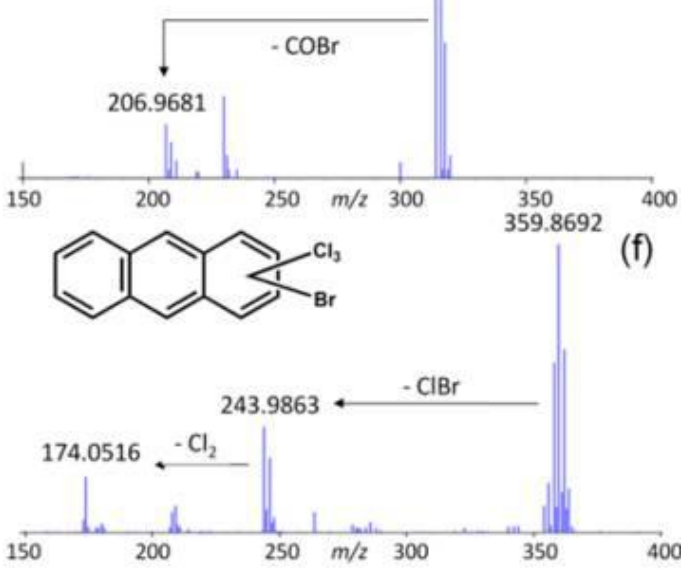

Figure 1.8 LEFT (a) Total ion and (b,c) Extracted ion chromatograms of the molecular ions of the (mixed) halogenated ANT/PHE and FLU/PYR isomers obtained from GC $\times$ GC-HRTOF analysis. RIGHT: HRTOF mass spectra with (d) $\mathrm{C}_{14} \mathrm{H}_{6} \mathrm{C}_{14} \bullet+$; (e) $\mathrm{C}_{12} \mathrm{H}_{5} \mathrm{OC}_{12} \mathrm{Br} \bullet+$; and (f) $\mathrm{C}_{14} \mathrm{H}_{6} \mathrm{Cl}_{3} \mathrm{Br} \bullet+$ molecular ions. The structures are tentatively proposed on the basis of exact mass measurements and EI dissociation behavior. Reprinted with permission from Ref. [63]. Copyright (2014) American Chemical Society. 
New software tools developed by Ubukata et al. [65] using non-standard Kendrick mass defect plots and accurate mass information were applied to non-targeted analysis of dust samples collected from an electronic waste facility. The dust extract was analyzed by GC×GC-HRTOFMS using both electron ionization (EI) and electron-capture negative-ion (ECNI) modes. Kendrick plots constructed using the accurate mass data with non-traditional mass scales, such as $-\mathrm{H} /+\mathrm{Cl}(34 / 33.96102),-\mathrm{H} /+\mathrm{Br}(78 / 77.91051), \mathrm{CF}_{2}(50 / 49.99681)$, and $+\mathrm{F} /-\mathrm{Cl}(16 / 15.97045)$ substitutions, were used to facilitate the identification of $\mathrm{Br}, \mathrm{Cl}$, and $\mathrm{F}$ containing compounds. EI and ECNI mass defect plots were very similar, ECNI producing more intense molecular ions in some instances. This approach, combined with NIST library database searches to confirm the identifications, led to the identification of tris-(1-chloro-2-propyl) phosphate, polychlorinated terphenyls, polychlorinated biphenyls, tetrabromobisphenol A, polybrominated diphenyl ethers, and hexabromobenzene.

Antle et al. [66] reported for the first time the use of spectral deconvolution of full scan quadrupole GC $\times$ GC-qMS data for the quantification of 16 EPA priority pollutant PAHs and polycyclic aromatic sulphur heterocycles (PASH) in coal tar contaminated soil and sand samples. PAH and dibenzothiophene standards were analyzed by GC×GC-qMS and GC-qMS to determine linear ranges $(10-3000,25-6000$ and $50-6000 \mathrm{ng} / \mathrm{mL}$ depending on the target compounds for $\mathrm{GC} \times \mathrm{GC}$ ) and to calculate LODs of the method (ranging from 5.5 to $35.8 \mathrm{ng} / \mathrm{g}$ for $\mathrm{GC} \times \mathrm{GC}$ ). LOQs ranged from 10-50 ng/mL. Due to a lack of standards, alkylated PAH homologues were quantified using each parent's average RF. Alkyl PASH concentrations were calculated using dibenzothiophene response factor. Spectral deconvolution algorithms were developed to process the GCxGC-qMS data. The deconvolution software automatically integrated the peak areas. For the homologues where multiple fragmentation patterns were needed to capture all isomers, the 
software integrated and summed peaks by pattern. PAH and PASH concentrations in the sediment samples were measured using both systems to assess the data quality differences, results showing good agreement between the two. The relative percent differences ranged from 0 to $217 \%$, while some homologues were detected only by $\mathrm{GC} \times \mathrm{GC}$ as it offered improved separation and sensitivity.

\subsubsection{Organohalogens and Pesticides}

Quantification of PCDD/Fs by GC $\times$ GC-HR-TOFMS in crude extracts of municipal waste incinerators fly ash and flue gas was reported for the first time by Hashimoto et al. [67]. The fly ash extracts were analyzed with and without cleanup (to simplify the method) on InertCap5MS/Sil $\times$ InertCap-17MS/Sil column set. Separation of the isomers with TEF factors was achieved in this study, in contrast to the classical one dimensional analysis (e.g. 2,3,4,6,7,8hexachlorinated dibenzofuran separated from co-eluting isomer $1,2,3,6,8,9-\mathrm{HxCDF}$ on a $5 \mathrm{MS}$ column). Instrument bundled software and in-house developed macros were used to transfer, process and quantify the results for the determination of chlorinated dioxins and furans that present TEF. Quantitation was based on relative calibration method, and the results obtained for the real samples with this system were comparable to those obtained from the classical GCHRMS analysis. The advantages of using GC $\times$ GC-HR-TOF-MS over ${ }^{1} \mathrm{D}-\mathrm{GC}$ or low resolution TOF were emphasized in this study as well: the target compounds were separated and accurately quantified in the extract without clean-up resulting in time savings; the high mass resolution contributed to reduction of interferences; other TEF congeners separated in ${ }^{2} \mathrm{D}$ plane could be quantitated. The disadvantage of the GC×GC-HR-TOF-MS system was the narrower dynamic range compared to GC-HRMS, which proved insufficient to quantitate environmental pollutants 
present at various concentrations. In addition, the amount of data collected was overwhelming. These disadvantages were addressed in a follow-up study [68]. The new system consisted of a GC $\times$ GC-MS/MS equipped with InertCap5MS/SN $\times$ BPX-50 column set and cryogenic modulator. The MS/MS used the neutral loss scan mode to comprehensively and selectively detect halogenated compounds in environmental samples. The standards of 211 halogenated compounds (16 PCDDs, 19 PCDFs, 62 PCBs, 39 PBDEs, 21 chlorinated PAHs, 11 brominated PAHs and 43 other POPs) were measured in MS/MS neutral loss mode to optimize the method. The mixed halogens were identified by overlapping neutral loss scan 2D chromatograms. The method was applied to environmental samples where a large number of peaks containing halogens were detected: some of the compounds were identified as PCBs, PCDDs and PCDFs, but many remained unidentified due to lack of sensitivity and/or library search mismatch. Some peaks overlapped in the 2D chromatograms for ${ }^{35} \mathrm{Cl},{ }^{79} \mathrm{Br}$ and ${ }^{19} \mathrm{~F}$ neutral loss scans, suggesting that mixed halogenated compounds were present in the samples but not identified. The authors concluded that the instrument still lacked in sensitivity compared to 1D-GC; however, the approach using the enhanced $\mathrm{GC} \times \mathrm{GC}$ resolving power and monitoring neutral loss scan with MS/MS was very effective to selectively detect halogens.

GC $\times$ GC-HRTOF-MS along with newly developed software for data processing were used by Hashimoto et al. [69] to selectively detect and identify trace levels of organohalogens in fly ash, soil, sediment, the atmosphere and human urine. As compared to other software developed by different groups/companies, this software could work standalone on Microsoft Windows and read netCDF formatted high resolution data. The software extracted sub-sets of GC $\times$ GC-HRTOFMS data containing only the mass spectra of organochlorine and organobromine isotopic patterns. It was also able to vary parameters such as mass accuracy, mass range, threshold of signal 
intensity and margin of error of the theoretical isotopic ratios of $\mathrm{Cl}$ and $\mathrm{Br}$, as well as to prescreen data by checking for mass defect. The mass resolution played an important role in the accuracy of the results, working as a mass filter. The mass defect filtering was very effective in removing the mass spectra of hydrocarbons when indoor air sample was analyzed, but not in removing the mass spectra of molecular sulfur in sediment and human urine samples. The software combined with NIST search reduced the number of peaks from over 3,000 to 73 for indoor air sample, 50 of them being identified.

Zushi et al. [70] developed an automatic peak sentinel tool (T-SEN) to accurately identify and quantify 17 PCDD/Fs and 24 PCBs in sediment certified reference material sample. The tool, consisting of an algorithm for peak finding and peak shape identification, allowed rapid screening, identification and quantitation of target compounds (user database) in large data sets acquired from GCxGC-HRTOF-MS in less than $20 \mathrm{~min}$. The method was also evaluated by GC $\times$ GC-qMS to compare the results obtained. The database was prepared for the target compounds as well other POPs and BDEs using both $\mathrm{GC} \times \mathrm{GC}$ systems and contained information on the ${ }^{1} \mathrm{D}$ and ${ }^{2} \mathrm{D}$ retention times, fragment ions and relative ratios of the target ions. In addition, an algorithm was developed to correct the retention times of the target compounds using an internal standard, so that interferences and/or overlap from other POPs could be eliminated. The authors pointed that using the ionic liquid stationary phase, SLB-IL59, in the second dimension, resulted in better separation of PCB congeners than BPX-50 (polar column). The method was applied to both standards and an ASE-certified reference sediment extract, all the targeted PCDD/Fs and PCBs being successfully identified. Similarly, most of the POPs and BDEs were identified with a few exceptions. The authors concluded that further software modification should be investigated. When the two systems were compared, GC×GC-HRTOF-MS resulted in accurate 
assignment for the PCDD/Fs and PCBs (except for PCB123/PCB107/PCB109 co-elution) while GC $\times$ GC-qMS incorrectly assigned peaks due to the contribution of matrix-derived ions present in all scan records, a disadvantage when using low mass resolution. The calculated concentrations of the target compounds in CRM sample by using GC×GC-qMS were much higher than the certified values for most of the compounds due to incorrect assignment. The concentrations of PCBs 77, 105 and 118 were lower than the certified values due to the mass errors in the target ions caused by interferences and/or incorrect assignment of internal standards. The differences between the values measured by GC $\times \mathrm{GC}-\mathrm{HRTOF}-\mathrm{MS}$ with $\mathrm{T}-\mathrm{SEN}$ and the certified values for the certified reference material ranged from 7.3 to $36.9 \%$ for compounds with concentrations above the limit of quantification. False positives/negatives were not observed, except when coelutions occurred. GC $\times$ GC-HRTOF-MS in combination with T-SEN proved to be a powerful approach for comprehensive analysis. In a follow-up study, Zushi et al. [71] developed data processing tools for non-target analysis using $\mathrm{GC} \times \mathrm{GC}-\mathrm{HRTOF}-\mathrm{MS}$. The method was then applied to a sediment core to identify the chlorinated compounds present. The data processing tools were used in two different modes: a qualitative non-target screening using mass defect filter followed by artificial neutral loss scan (MDF/artificial NLS), and a semi-quantitative target screening method using Entire Domain Combined Spectra Extraction and Integration Program (ComSpec) and two-dimensional peak sentinel (T-SEN). MDF and artificial NLS were developed for detecting halogenated compounds and were presented in previous studies discussed in this review [67,69]. Higher mass accuracy and resolution allowed the results of the NLS tool to be more accurate. Mass defect filtering separated heteroatoms-containing compounds from hydrocarbons. T-SEN software was developed to find peaks and identify their peak shape, resulting in rapid screening of target compounds (custom built database containing 170 
chemicals) for large data sets. The principle of ComSpec is similar to that of T-SEN, but works without the retention time information and extracts all the isomers from the entire chromatogram. Non-target screening was performed on sediment core. The results showed that when applied together, MDF and artificial NLS worked effectively to remove most of the interferences in the matrix-rich chromatogram (column bleed and terpenoid compounds were not removed by MDF or NLS only). Several peaks were assigned using the accurate mass database. In addition, compounds like DDT, DDD and DDE deleted by MDF were confirmed as being present in the sample by ComSpec (Figure 1.9 - [71]). Semi-quantitation performed with T-SEN and ComSpec had the advantage of being able to automatically quantitate individual compounds and groups of target analytes. The performance of this method was evaluated by comparing PCB congeners results with those obtained from GC-HRMS target analysis. 


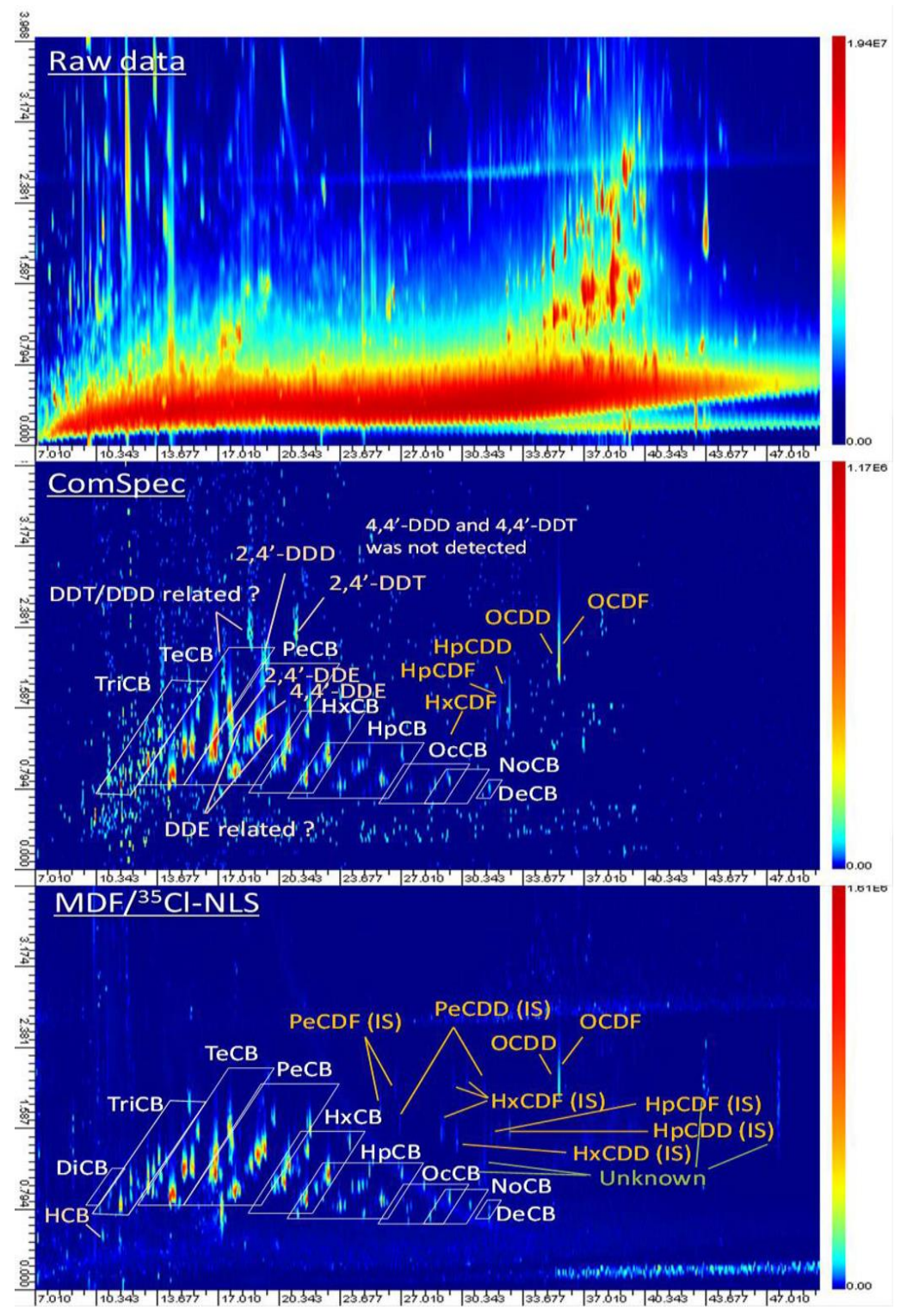

Figure 1.9 Comparison of the results obtained from different data processing methods. Upper: raw data. Middle: ComSpec (tolerance margin of 0.03 Da). Lower: consecutive use of MDF (mass defect $0.2 \mathrm{~m} / \mathrm{z}$ ) followed by ${ }^{35} \mathrm{Cl}-\mathrm{NLS}(\Delta \mathrm{m} / \mathrm{z} 34.969)$. Reprinted from Ref. [71], Copyright (2014), with permission from Elsevier.

In a recent study, Zushi et al. [72] developed an alignment algorithm for correcting retention time shifts in $\mathrm{GC} \times \mathrm{GC}-\mathrm{HRTOF}-\mathrm{MS}$ data to account for run-to-run variations due to 
variations in instrumental conditions. The RT shift correction algorithm included pixel-by-pixel correction and was able to handle high mass resolution data. The algorithm was successfully applied to a sediment sample analyzed on a damaged column.

By using GC×GC-TOFMS, de Vos et al. [73] established an alternative method to GCHRMS for the quantitation of PCDDs and PCDFs in different environmental matrices as well as for environmental forensic investigations [74]. The instrument was tuned on the $\mathrm{m} / \mathrm{z} 414$ ion to improve the signal intensity at the higher mass range. The target compounds were separated on three different column sets to assess the selectivity. The Rxi-5Sil MS $\times$ Rtx-200 column combination was optimized for the separation of all the priority pollutants and was the one selected for the quantitation of TCDD/Fs in different samples. The limits of detection of the method of 300 fg on column for spiked soil samples were determined using Rxi-XLB $\times$ Rtx-200 column set, which provided better separation of individual congeners, hence more confidence in calculating the individual contributions of the seventeen priority PCDD/Fs towards total toxic equivalency $\left(2,3,7,8-\mathrm{TCDF} / 2,3,7,8-{ }^{13} \mathrm{C}_{12}\right.$-TCDF and the $2,3,7,8-\mathrm{TCDD} / 2,3,7,8-{ }^{13} \mathrm{C}_{12}$-TCDD separation with the XLB phase has been achieved with no overlapping interference from the other $\mathrm{TCDD} / \mathrm{F}$ isomers present). The recoveries for the target compounds ranged from 69 to $110 \%$. The excellent separation achieved when using Rxi-XLB $\times$ Rtx-200 along with the validation results showed that the method presented in this study met the US EPA 1613 criteria for PCDD/Fs testing and was a more affordable alternative to GC-HRMS [64].

Rimanyi et al. [75] assessed the distribution of 2,3,7,8-PCDD/Fs in sediment samples collected from sites previously identified as POP hotspots. The extracts pre-screened for dioxinlike activity using bioassay tests (DR-Luc) were analyzed by GC×GC- $\mu \mathrm{ECD}$. The system was calibrated for 7 PCDDs and 10 PCDFs. The method's LOQs for PCDD/Fs varied between 0.13 
$\mathrm{pg} / \mathrm{g}$ for $2,3,7,8-\mathrm{TCDF}$ to $5.2 \mathrm{pg} / \mathrm{g}$ for OCDD, while the average LOQ value (based on $10 \mathrm{x}$ signal to noise ratio) for DR-Luc analyzes was $0.72 \mathrm{pg} T E Q / \mathrm{g}$. All sediment samples tested positive for dioxin-like activity. Further confirmatory $\mathrm{GC} \times \mathrm{GC}-\mu \mathrm{ECD}$ analysis revealed the presence of 11 $\mathrm{PCDD} / \mathrm{Fs}$ and $6 \mathrm{PCDD} / \mathrm{Fs}$ in sediment samples collected from the two locations. A direct comparison of the dl-potency calculated from GC $\times \mathrm{GC}-\mu \mathrm{ECD}$ measured compounds and the dlpotency acquired from the bioassay DR-Luc was not possible as other POPs (e.g. PCBs) were not targeted.

Standard methods for the analysis of halogenated contaminants in soil and sediment samples involve traditional $1 \mathrm{D}-\mathrm{GC}$ coupled with different detectors such as $\mu \mathrm{ECD}$, qMS and HRMS. GC $\times$ GC- $\mu \mathrm{ECD}$ proved to be a suitable technique for the analyzes of PCB and pesticides and other non-target compounds in soil/sediment samples [27-28,76-78]. In one of their earlier work, Muscalu et al. [76] developed a method to separate PCBs, organochlorine pesticides and chlorobenzenes. The results obtained on the column set selected, DB-1×Rtx-PCB, showed the improved separation of 118 target compounds ( 80 PCBs, 23 OCs and $15 \mathrm{CBs}$ ) when using this technique. Similar to previous studies [48,79-81], ordered structure was also observed in the 2D plane for structurally related compounds when using this column set. PCBs were separated as bands in the second dimension plane according to their degree of chlorination, as well as their planar structure, with mono-ortho and non-ortho PCBs eluting later in the second dimension due to the selectivity of Rtx-PCB stationary phase for compounds with planar configuration. Following up on this study, a routine accredited method was developed and validated [27-28]. Quality control and quality assurance procedures, implemented as per ISO 17025 requirements, showed that the method performed very well. By implementing this method for routine work, one GC $\times$ GC- $\mu$ ECD instrument replaced four different GC-ECD systems that were using seven 
column phases to analyze multiple fractions of each sample extract (Figure 1.10). Since its implementation in 2010, GC×GC- $\mu$ ECD method combined with GC×GC-TOFMS for further characterization proved to have many benefits, including identification of non-targeted compounds and re-directing the samples to validated methods for the identified compounds when available (e.g. BDEs, PCNs), tracking down the source of contamination based on Aroclor matching, identifying the source of DDT contamination when dicofol was detected, or identifying SCCPs as ubiquitous contaminant class in sediment samples. This method was also tested with a newly developed consumable-free single-stage thermal modulator (See Chapter 2 and Reference [77]). The results were very encouraging, as negligible shifts in retention times were observed for both within-day and day-to-day comparisons of the studied sediment samples, and the quantitative results were comparable. Overall, the routine analysis and quality control applications would benefit from the improved reproducibility by using this modulator, as the variances in cold/hot jet flows and temperatures were eliminated. 

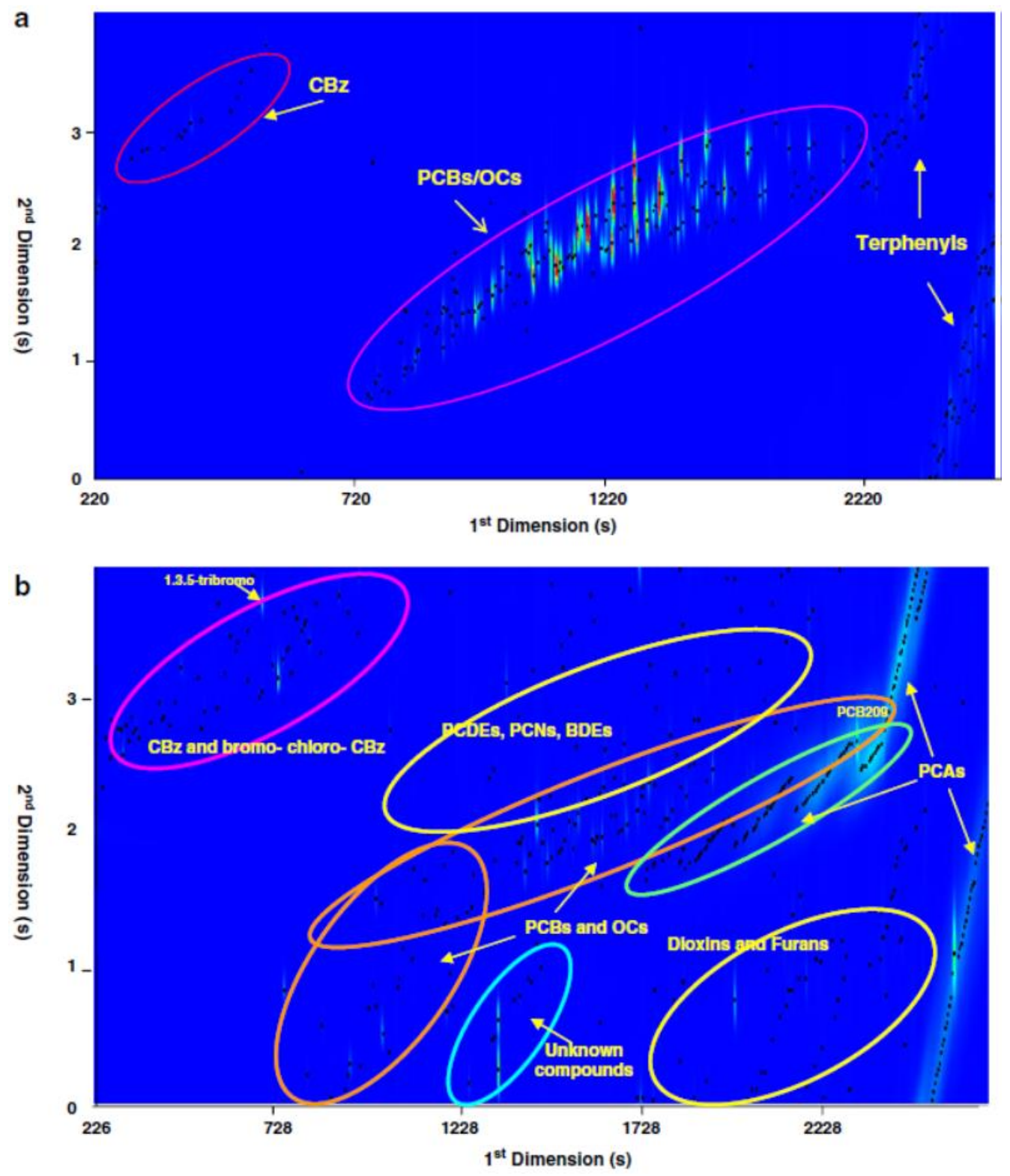

Figure 1.10 a. Sludge sample analyzed by $\mathrm{GC} \times \mathrm{GC}$ showing the presence of polychlorinated terphenyls along with the method's target compounds. b. Two-dimensional chromatogram of a sediment sample showing the presence of other contaminant classes in addition to the PCBs/OCs/CBz. Reprinted by permission from Springer Nature: Ref. [28], Copyright (2011).

In another study [78], seven pesticides were monitored in sediment samples by using $\mathrm{GC} \times \mathrm{GC}-\mu \mathrm{ECD}$ : propanil, fipronil, propiconazole, trifloxystrobin, permethrin, difenoconazole 
and azoxystrobin. Two column combinations were tested, DB-5×DB-17MS and HP-50+xDB1MS. The first combination achieved the best results and was further used for quantitation. The method's LODs were lower than those obtained for the ${ }^{1} \mathrm{D}$ GC method, results showing that coeluting matrix compounds known to bias high the ${ }^{1} \mathrm{D}-\mathrm{GC}$ results (e.g. trifloxystrobin) were separated by $\mathrm{GC} \times \mathrm{GC}$, hence improving the accuracy of the data.

Naudé et al. [82] used off-line heart-cut GC-TOF-MS and GC×GC-TOFMS to develop a method for enantiomeric analysis of o,p'-DDT and o,p'-DDD in contaminated soil and air. The enantiomer-specific determination is important for both health reasons and for forensic considerations to determine if the source of contamination is old or new. The authors proposed a solventless PDMS extraction for soil samples followed by thermal desorption GC-FID for separation. Similarly, a denuder device containing a micro quartz fiber filter/multichannel PDMS traps was used to sample air. An off-line heart-cut procedure was performed using a TD-GC-FID on a nonpolar column (Zebron ZB-1), with only the o,p' isomers of DDT and DDD fractions selectively collected on a multichannel open tubular PDMS trap. The collected fractions were injected in a GC-TOF-MS system using a chiral column in the first dimension (BGB-15) and an intermediate polarity column (Rtx-200) in the second dimension (not modulated, analyzed in 1D mode). The same GC-TOFMS system was used in 2D mode, to analyze the o,p'-DDT and o,p'DDD enantiomers without the heart-cut procedure. During conventional 1D chiral separation, o,p'-DDT enantiomers cannot be separated from p,p'-DDD peak, which is achievable by both heart-cut GC-TOF-MS and GC×GC-TOF-MS. The calculated enantiomeric fractions from both analyzes showed that the two systems produced results that did not differ significantly. The calculated enantiomeric fractions for o,p'-DDT and o,p'-DDD in soil samples gave an indication 
that both recent and historical sources contributed to the soil contamination. In addition, GC×GCTOF-MS demonstrated increased sensitivity compared to the heart-cut technique.

Fernandes et al. [83] compared the residue profiles of strawberries and soil samples collected from organic and integrated pest management farms. The samples were extracted using the QuEChERS procedure and cleaned-up by dSPE. Three different techniques were applied to cover a wider range of compounds: 27 target pesticides analyzed by LC-MS/MS (quantitative), 143 target pesticides analyzed by low pressure GC-MS/MS (quantitative), and over 600 screened pesticides by GC $\times$ GC-TOF-MS with an untargeted approach. Automatic compound detection was performed by ChromaTOF software using a custom-made pesticide library, similarity $>600$, and retention times within $20 \mathrm{~s}$ of the reference $t_{R}$ in the database. Qualitative screening of the pesticides by all three techniques was performed, showing that some pesticides were detected only by TOF or only by MS/MS, possibly due to the different sensitivity and selectivity of the two instruments. No pesticides were detected by any of the methods in the organic farm samples. Nine pesticides were detected by GC methods in samples collected from integrated pest management farms. The pesticides cyprodinil, fludioxonil and fluazifop-p-butyl found in soil samples were also detected in the corresponding strawberries, while pyrethroid fenpropathrin, detected in strawberries, gave the highest residue value. GC×GC-TOF-MS was used to identify additional pesticides (oxyfluorfen, tetraconazole) and provide a confirmation of those already found (iprodione, mepanipyrim, fludioxonil, fenhexamid, cyprodinil). All determined pesticide concentrations were below the European Union regulatory limits. The authors concluded that by using this approach, screening, identification, quantification and confirmation were possible, further emphasizing that pesticide monitoring should survey all pesticides in the sample, an untargeted approach made possible by GC×GC-TOF-MS. 
Zhu et al. [84] used GCxGC-NCI-MS to determine the three indicator toxaphene congeners (P26, P50 and P62) in soil samples. Toxaphene, a broad spectrum chlorinated pesticide consisting of several hundred congeners, is very difficult to analyze in environmental samples due to its complexity and the different response factors observed for each congener. The GC method was optimized using DB-XLB $\times \mathrm{BPX}-50$ column set to achieve the best separation of the individual congeners from the toxaphene technical mix. This method was applied to real soil samples known to be contaminated with toxaphene. All three indicator toxaphenes were detected, separated in the 2D space from the rest of the congeners present and quantitated in the soil samples. The use of $\mathrm{GC} \times \mathrm{GC}$ provided improved separation of the target compounds and made is possible to report toxaphene congeners as compared to a "total" toxaphene approach.

Organophosphorus pesticides and organophosphate esters were analyzed by GC×GCFPD (P-mode) in soil using a method developed by Liu et al. [85]. Different column sets were tested to achieve the best separation of the 37 target compounds, with HP-5×DB-1701 set found to provide better separation than SolGel Wax $\times \mathrm{HP}-5$ (upper temperature limit of $280{ }^{\circ} \mathrm{C}$ ) and $\mathrm{HP}$ $5 \times \mathrm{IL}-59$ (poor response for polar compounds). The $\mathrm{GC} \times \mathrm{GC}-\mathrm{FPD}(\mathrm{P})$ proved to be an accurate, sensitive and simple method for P-compound analysis in complicated environmental samples.

\section{Other Compounds}

Fatty acid methyl esters and bacterial fatty acid methyl esters were analyzed in environmental samples by GC×GC-MS/FID equipped with different modulators: flow modulator [86-87] and Everest model longitudinally modulated cryogenic system [88]. A number of column sets were evaluated for the isomeric separation of fatty acid methyl esters. Using a nonpolarxpolar ionic liquid column combination, excellent group-type separation of fatty acids with different carbon numbers and number of unsaturations was obtained, providing information that 
was complementary to GC-MS identification [87]. Zeng et al. [88] developed a method for profiling the phospholipid fatty acids in soil samples. Due to the better separation offered by GC $\times$ GC, clarification of fatty acids with complex structures (e.g. hydroxyl, cy-branched, epoxy) was possible. In addition, uncommon oxygenated FAMEs were found in high abundance and were further characterized by $\mathrm{GC} \times \mathrm{GC}-\mathrm{qTOF}-\mathrm{MS}$ (Figure1.11). A more recent study used GC $\times$ GC-MS for lipidomic investigations to highlight differences in the fatty acid profiles of fish samples from aquaculture and from the wild [89]. 

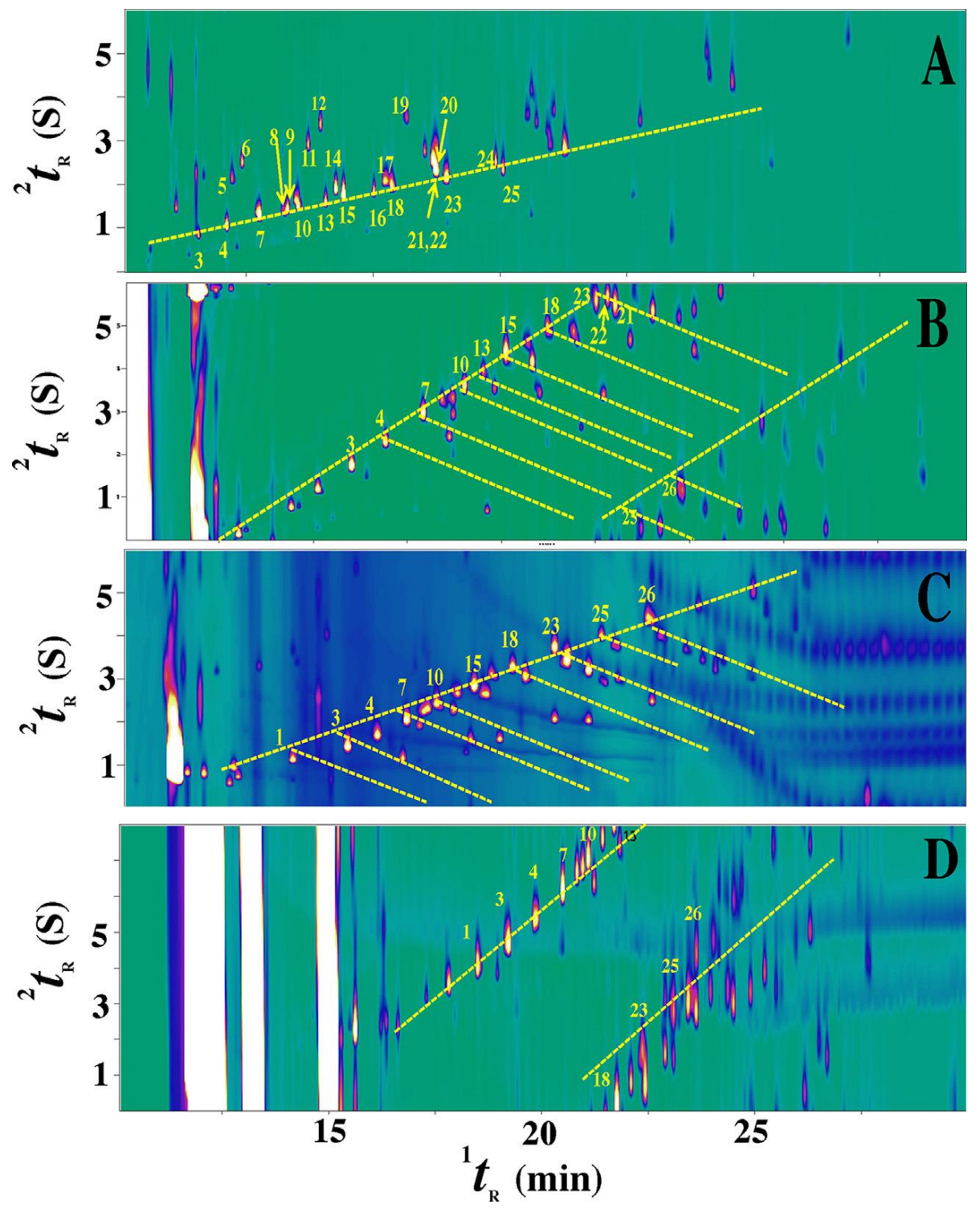

Figure1.11 GC $\times$ GC-FID contour plots of 100 ppm FAME and BAME mixtures on GC column sets (A) HP-5ms $\times$ HP-INNOWax; (B) HP-88×HP-INNOWax; (C) SolGelWAX×Rxi-5silMS; and (D) IL111×IL59. Reprinted from Ref. [79], Copyright (2013), with permission from Elsevier.

Jacobs et al. [90] used a newly developed miniaturized single-stage thermal modulator to evaluate the modulation of a range of compounds with different functionalities, and then applied it to the analysis of petroleum hydrocarbon spills to evaluate the ongoing remediation efforts. The 
modulator incorporated an adsorptive stationary phase coating and high speed resistive heating for comprehensive two-dimensional modulation. Compounds with different functional groups were used to determine if any solute discrimination occurred in the modulator. All compounds were modulated with the exception of n-octane (boiling point $125^{\circ} \mathrm{C}$ ); toluene with a lower boiling was focused by the modulator. The authors suggested that it was due to the properties of the stationary phase of the trap. Polar solutes were retained more strongly by the seconddimension column than aliphatic species, with greater peak broadening observed. The authors concluded that the performance of this consumable-free system was comparable to that of the other thermally modulated devices. The GC×GC-FID system, calibrated using Special Antarctic Blend diesel, was used to analyze petroleum hydrocarbons in contaminated soil sample extracts. Principal component analysis of GC $\times$ GC-FID chromatograms also proved to be an effective tool to categorize and fingerprint the samples in this study.

Analyzing chlorinated paraffins in environmental matrices is very challenging. GC $\times \mathrm{GC}$ already proved to be a powerful technique to separate and characterize $\mathrm{CP}$ mixtures in different studies [91-94]. Using a novel GC×GC-ECNI-HRTOF-MS method, short chain chlorinated paraffins (SCCPs) and medium chain chlorinated paraffins (MCCPs) were profiled and quantified in environmental samples by Xia et al. [92]. The method was optimized to achieve the best orthogonal separation of formula congener groups, to separate SCCPs from MCCPs, and to separate both SCCPs and MCCPs from the co-extracted matrix components. For a technical SCCP and MCCP mixture, over 2300 peaks could be distinguished in the GC $\times$ GC chromatogram using the optimized parameters. The 2D chromatograms contained ordered structures on diagonal lines, each structure formed by a group of CPs with the same number of carbon plus chlorine atoms. The quantification procedure was based on a method that compensated for different total 
response factors caused by samples and standards with different $\mathrm{Cl}$ contents. For each $\mathrm{CP}$ formula congener with 5-10 chlorine atoms, the highest most abundant $[\mathrm{M}-\mathrm{Cl}]^{-}$ion was used as a quantification ion and the next most abundant ion was used as a qualification ion. The method allowed $48 \mathrm{CP}$ formula congener groups (C10-17 and $\mathrm{Cl} 5-10$; di-/tri/tetra-Cl had low responses and were not included) to be analyzed highly selectively in one injection through accurate mass measurements of the $[\mathrm{M}-\mathrm{Cl}]^{-}$ions in full scan mode (Figure 1.12). The quantification procedure combined with the group analysis strategy were used to build calibration curves between the total response factor for a $\mathrm{CP}$ mixture and the calculated chlorine content for five CPs with different chlorine content (53.5-59.25\% for SCCPs and 44-57\% for MCCPs). The method detection limits were lower than or comparable to those achieved using HRGC-ECNI-HRMS. New compounds, with the formulas $\mathrm{C}_{9} \mathrm{H}_{14} \mathrm{Cl}_{6}$ and $\mathrm{C}_{9} \mathrm{H}_{13} \mathrm{Cl}_{7}$, were found in sediment and biological samples for the first time. Other POPs were investigated to assess if any of them would interfere with CPs. The resolution offered by $\mathrm{GC} \times \mathrm{GC}$ allowed the CPs to be separated clearly from the other classes of compounds in full scan mode. The method was shown to be a powerful tool for the analysis of CPs in environmental samples.

Muscalu et al. developed a quantitative method for the analysis of SCCPs in sediment samples by using GC $\times$ GC- $\mu$ ECD (See Chapter 3 and Reference [94]). Distinct ordered bands were observed in the $\mathrm{GC} \times \mathrm{GC}$ chromatograms pointing to group separation. Complete homologue separation was not possible with the DB-1×Rtx-PCB column combination used due to the complexity of the SCCP mixtures. Using the Classification function of the ChromaTOF software, classifications were created for each band and summary tables were generated to determine total area counts. As the ECD provided only retention time information and no mass spectral information, quantitation masses could not be selected for each class, and calibration curves could 
not be generated through the ChromaTOF software. The summary table was exported to Excel where linear calibration curves were created and then applied to quantify all of the standards. To further validate the proposed method for the analysis of SCCPs in soils and sediments, the laboratory participated in inter-laboratory studies for the analysis of standards and sediment samples, demonstrating that the method was suitable for this purpose. The authors also noted a few drawbacks of this procedure: SCCPs could not be fully separated from MCCPs, quantitation was not possible in this case, and data processing was very time consuming due the limitations in the software. The advantage of using GC $\times \mathrm{GC}-\mu \mathrm{ECD}$ for routine environmental analysis is its screening power: 2D chromatograms of samples analyzed routinely for other halogenated compounds such as PCBs could be sorted and data further processed when SCCPs were present. 


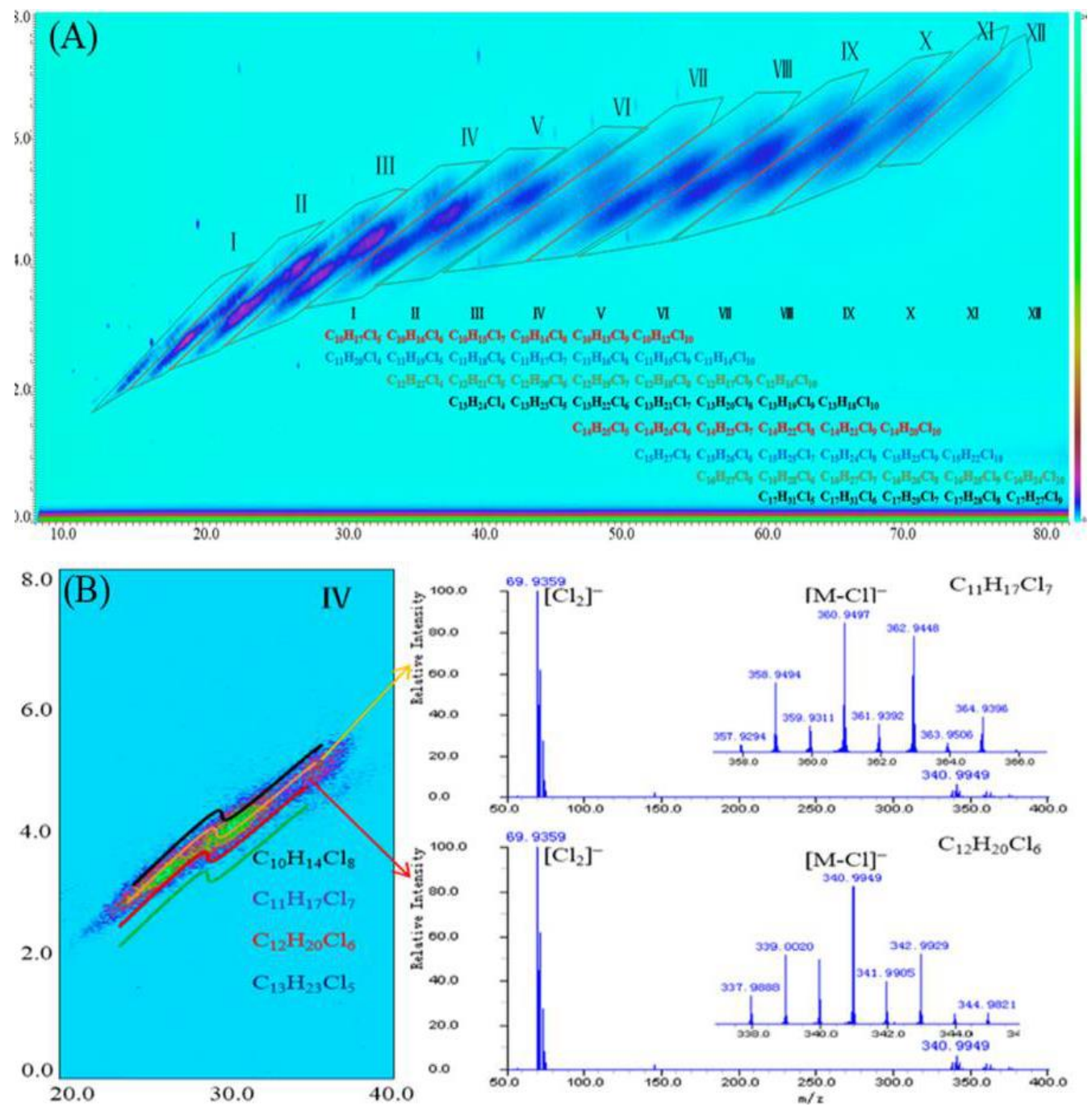

Figure 1.12 (A) GC $\times$ GC-ECNI-HRTOF-MS TIC chromatograms and GC $\times$ GC elution pattern for a mixture of a technical $51.5 \% \mathrm{Cl}$ SCCPs mixture and a technical $52 \% \mathrm{Cl}$ MCCPs mixture (lower right: molecular formulas of the $\mathrm{CP}$ congeners in each group). (B) Extracted ion chromatograms (left: group IV containing $\mathrm{CP}$ formula congeners of $\mathrm{C}_{10} \mathrm{H}_{14} \mathrm{Cl}_{8}, \mathrm{C}_{11} \mathrm{H}_{17} \mathrm{Cl}_{7}$, $\mathrm{C}_{12} \mathrm{H}_{20} \mathrm{Cl}_{6}$, and $\mathrm{C}_{13} \mathrm{H}_{23} \mathrm{Cl}_{5}$ ) and accurate mass spectra (right) of selected peaks for CPs analyzed using the GC $\times$ GC-ECNI-HRTOF-MS. Reprinted with permission from Ref. [92]. Copyright (2016) American Chemical Society. 


\subsubsection{Multiresidue Methodologies}

The powerful chromatographic resolution of $\mathrm{GC} \times \mathrm{GC}$ coupled with the automated mass spectral deconvolution of TOFMS allowed the separation of many constituents of previously unresolved complex mixtures of contaminants in sediment samples as presented by Skoczyńska et al [95]. The purpose of the study, as the authors stated, was not meant for routine analysis but to obtain a much more complete "chemical picture" and draw a "map" of different pollutant groups present in the sample. Using DB-5×007-65HT column set, over 400 compounds were tentatively identified from three different fractions. In fraction 1 (non-polar fraction), acyclic and cyclic alkanes, cyclo-S6 to -S8, PAHs and alkylated-PAHs, PCBs, chlorinated PAHs (e.g. monoand di-chlorinated naphthalenes and anthracenes or phenanthrenes) were tentatively identified. The second fraction was found to be more complex, containing a large group of aromatics such as monoaromatics, diaromatics, alkylated diaromatics, diaromatics with a heteroatom (dibenzothiophene, dibenzofuran) and their alkylated derivatives. The retention times in the second dimension depended on both the degree of aromaticity and the presence of heteroatoms with the following retention times order in the second dimension: $\mathrm{O}-\mathrm{C}-\mathrm{S}-\mathrm{N}$. Fraction 3 revealed the presence of oxygenated compounds (quinones and ketones), chlorinated and aminoanthraquinones. All fractions had many unidentified compounds as well. This type of analysis is important in environmental studies, as further elucidation of the toxicological properties of the samples might be provided.

Hilton et al. [96] developed a screening method for the identification of different classes of compounds that could be present in household dust, a potential major source of human exposure to environmental contaminants. Dust reference material (SRM-2585) extracted by ASE was cleaned up by gel permeation chromatography and analyzed on Rxi-5×BPX-50 column set 
installed in a GCXGC-TOFMS system. Automated identification of signature mass spectral patterns combined with location in the $\mathrm{GC} \times \mathrm{GC}$ chromatographic plane were used to identify compounds. Using the scripting feature in the LECO ChromaTOF software and user-written scripts, the resulting peak table was automatically filtered to identify analytes belonging to the following classes: PAHs, phthalates, halogen-containing compounds and nitro- compounds. The authors estimated that compounds at concentrations of $10-20 \mathrm{ng} / \mathrm{g}$ dust were classified by the scripts. The use of scripts allowed rapid identification of compounds of interests; however, further target analysis was required.

Pena- Abaurrea et al. [97] also applied the scripting procedure to prioritize data processing in sediment samples for the identification of potentially novel persistent and bioaccumulative halogenated chemicals. The script was based on the recognition of halogenated isotope cluster patterns allowing for the simultaneous detection of chlorinated, brominated, or mixed halogen substituted compounds in a single classification. The script applied a sequential evaluation of different mass variables to every peak identified: identification of the highest $\mathrm{m} / \mathrm{z}$ halogenated isotope cluster, evaluation of the relative abundances and evaluation of false positives corresponding to sulfur homologues. The script was validated by using different halogenated standards (120 standard containing $\mathrm{Cl}$ - and $\mathrm{Br}$ - compounds), then applied to real sediment samples (Figure1.13). Both manual review and halogen filter script were applied to interpret the data, the authors concluding that the estimated level of accurate identification of organohalogens by using the script ranged from 90 to $95 \%$ and was dependent on the relative S/N ratio (misidentification occurred when $\mathrm{S} / \mathrm{N}<10: 1$ and/or the matrix band interfered with the analyte peaks). Beside the known POPs, three decachlorinated dechlorane analogs, two undecachlorinated dechlorane species and a novel mixed chloro/bromocarbazole were identified 
in a number of sediments analyzed. The relative peak abundances for these compounds were in the same order of magnitude or slightly higher than levels observed for conventional POPs.

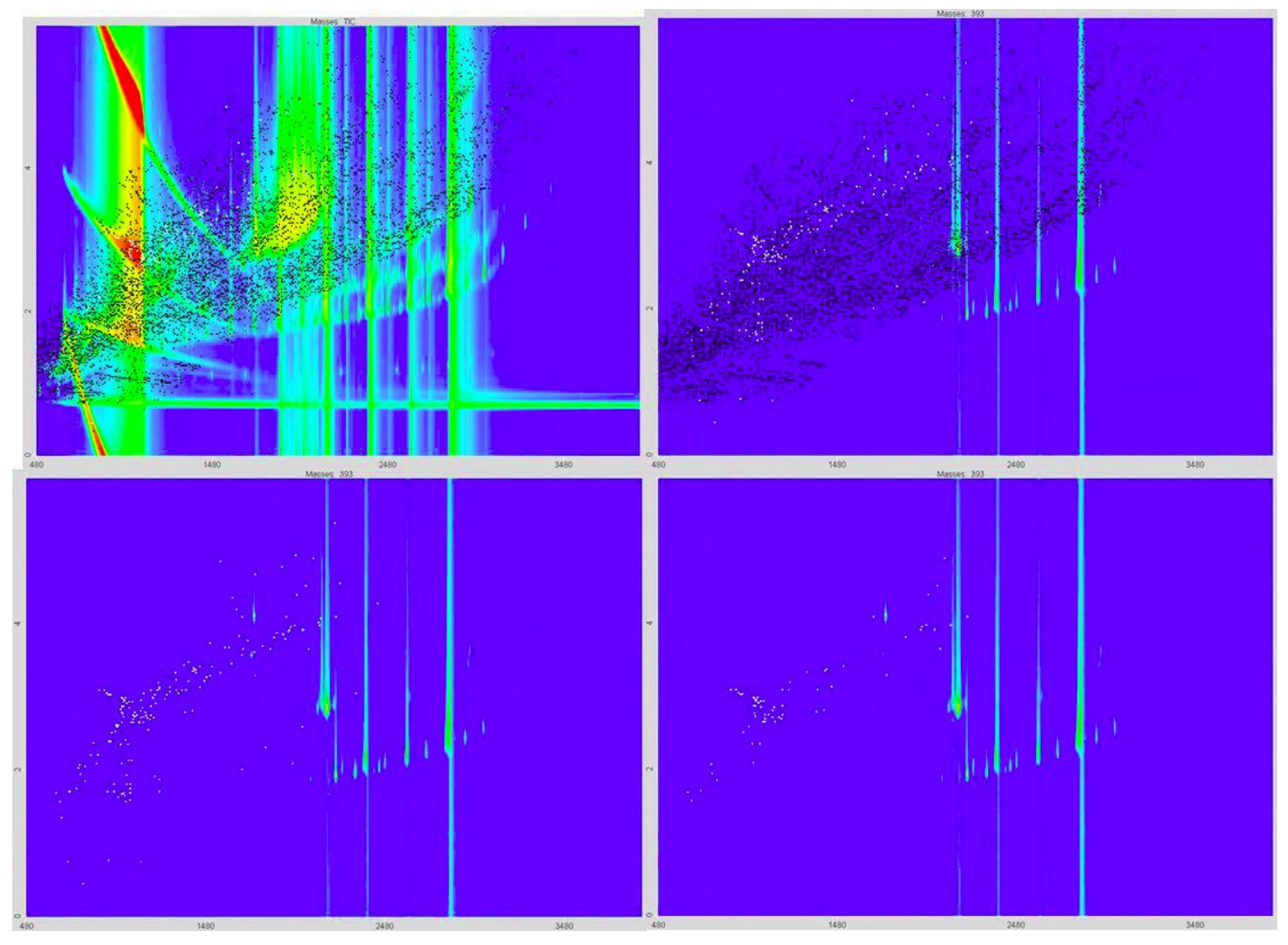

Figure1.13 Reconstructed contour plot for sample 31 (A) total ion mode, (B) extracted ion mode for $\mathrm{m} / \mathrm{z} 393$, (C) classified peaks in the extracted mode and (D) unknown classified peaks in the extracted mode after filtering well-known halogenated chemicals. Reprinted with permission from Ref. [97]. Copyright (2014) American Chemical Society.

As the authors stated, mixed chloro/bromocarbazoles were identified for the first time in environmental samples. This finding emphasized once more the importance of using $\mathrm{GC} \times \mathrm{GC}$ for identifying novel persistent halogenated contaminants and the enhanced benefits of using 
scripting classification to reduce the data processing time and minimize the human error during manual interpretation.

GC $\times$ GC-TOF-MS non-targeted screening and quantification strategies are increasing in popularity in the analysis of environmental samples. Zhang et al. [98] developed methods to analyze organic pollutants in soil samples collected from leaching basins. After method optimization, six main classes of compounds were detected: PAHs, phthalic acid esters, phenolic compounds, hydrocarbons, benzene derivatives and aliphatic acids. Based on the screening results, 50 compounds were quantitated and reported in the soil samples tested.

Veenaas et al. [99] developed a similar approach, using both non-targeted and quantitative methods for sludge samples analysis. Non-discriminating sample preparation methods were developed prior to GC $\times$ GC-HRTOF-MS instrumental analysis using PLE with in-line silica clean-up or off-line GPC clean-up. Taking both methods into account, more than 1500 compounds were found, over 190 were tentatively identified and 99 compounds were further quantitated (PCBs, PAHs, fragrances, $\mathrm{Cl} / \mathrm{Br}$-diphenyl ethers, other non-polar compounds). The non-targeted analysis revealed the presence of many classes of compounds primarily of anthropogenic origin (organophosphates, PPCPs, synthetic antioxidants, UV screens and stabilizers, pesticides and other chlorinated compounds, and process chemicals), some of them not previously reported (e.g. dichlorophenyl coumarins, dichlororflavones).

\subsubsection{Air and Aerosols}

The complexity of the atmospheric matrix is well known, as a large number of organic and inorganic compounds are present in the gas phase and particulate matter. Volatile organic 
compounds (VOCs) emitted from many anthropogenic and natural sources and having high reactivity play an important role in the formation of urban photochemical smog and tropospheric ozone. The exposure to air particulate matter can have negative effects on environment and humans, thus the importance of developing fast and reliable methods for monitoring of and identifying atmospheric pollutants. An early study published by Lewis et al. demonstrated the potential of $\mathrm{GC} \times \mathrm{GC}$ for air and aerosol analyzes by isolating and classifying more than 500 volatile organic compounds in urban air samples [100]. Past reviews summarized the advantages of using $\mathrm{GC} \times \mathrm{GC}$ in air analysis and its applications [4,101-103]. Table A.4 (Appendix A) illustrates more recent applications.

Following up on previous research [104-105], Fushimi et al. [106] reported a highly sensitive method for the simultaneous quantification of 29 target PAHs and PAH derivatives (oxygenated, nitrated, and methylated PAHs) in trace particulate samples by using TD-GC $\times \mathrm{GC}-$ MS/MS in selected reaction monitoring mode. The method could be applied to small sample amounts ( $\sim 20 \mu \mathrm{g}$ for atmospheric particles and $\sim 10 \mu \mathrm{g}$ for automobile exhaust particles), and the sensitivity achieved by TD-GC $\times$ GC-MS/MS was greater than that of TD-GC-HRMS and TDGC $\times \mathrm{GC}-\mathrm{qMS}$ by one or two orders of magnitude. Several compounds were excluded from the target compounds due to peak tailing and much smaller analytical sensitivities as compared to the other nitro-PAHs (6-nitrochrysene, dinitropyrenes, 6-nitrobenz[a]pyrene, and 3nitrobenzanthrone) or due to large variability in recoveries (fluorene, benzophenone, 2nitrofluorene). The method was applied to size-resolved diesel exhaust particles where major target compounds were successfully detected and quantified from accumulation-mode particles $(100-180 \mathrm{~nm})$ and from nanoparticles $(18-32 \mathrm{~nm})$. 
Manzano et al. [107] investigated the separation of complex PAH mixtures by using GC $\times$ GC-TOFMS. Four different column sets $($ Rtx-5MS $\times$ Rxi-17, Rxi-5MS $\times$ Rxi-17, LC-50×Rxi17 and LC-50×NSP-35) were tested to assess orthogonality and improve the separation of a standard solution containing 97 PAHs: parent PAHs, alkyl-PAHs, nitro-PAHs, oxy-PAHs, thioPAHs, high molecular weight PAHs ( $\mathrm{MW} \geq 300$ ), bromo-PAHs, and chloro-PAHs. The optimized methods were assessed for orthogonality by using the correlation between the retention times in both dimensions as an initial evaluation of the similarity between the column sets, then by using a procedure based on conditional entropy that considered the quantitative peak distribution in the entire 2D space. Chrysene and triphenylene were resolved when using LC-50 as first dimension column due to the separation mechanism depending on the molecular shape, while the use of the 2D space was maximized with the nanostationary phase column NSP-35. Atmospheric particulate matter, sediment and soil samples were analyzed to compare the four column combinations and assess the target compounds separation from the UCM. The UCM was distributed throughout the first dimension with Rtx-5MS $\times$ Rxi-17 and Rxi-5MS $\times$ Rxi-17, and distributed more in the second dimension with LC-50×Rxi-17 and LC-50×NSP-35 column sets, co-eluting with some early eluting PAHs (e.g. acenaphthene, acenaphthylene, 1-nitronaphthalene, 1,3- and 2,6- dimethylnaphthalenes). Soil and sediment samples analyzed on LC-50×NSP-35 indicated the presence of over 90 PAHs very well resolved from UCM. A follow-up study reported by Manzano et al. [108] quantified complex PAH mixtures in NIST SRMs: SRM 1650b diesel particulate matter (with clean-up) and SRM 1975 diesel particulate extract (with and without clean-up, Figure 1.14). Eighty-five PAHs (parent, alkyl-, nitro-, oxy-, thio-, bromo-, and chloro-PAHs) were quantified in those extracts. It was observed that naphthalene, 1-methyl and 2-methylnaphthalene showed weak interaction with the liquid crystal column and eluted with the 
solvent peak. Tailing was observed for polar-PAHs and more compounds were identified in the non-cleaned extract. In this study, the authors reported for the first time the presence of compounds not reported previously by NIST. 

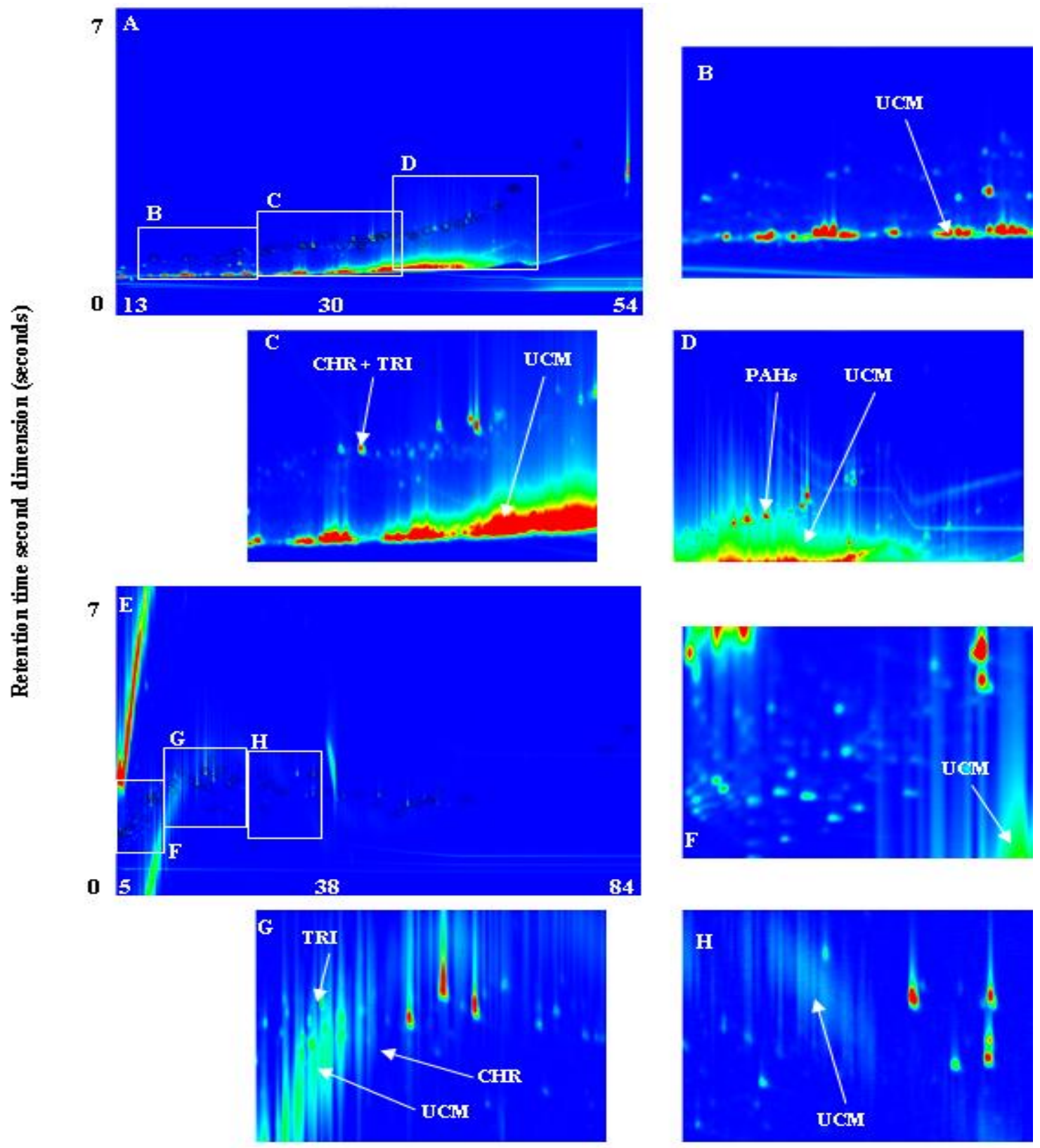

Retention time first dimension (min)

Figure 1.14 TIC for the analysis of NIST SRM1975: Diesel particulate extract, (A) using column combination Rtx-5MS $\times$ Rxi-17, (B), (C), (D) Portions of chromatogram shown in A. (E) using column combination LC-50×NSP-35, (F), (G), (H) Portions of chromatogram shown in E. Reprinted from Ref. [108], Copyright (2013), with permission from Elsevier. 
$\mathrm{GC} \times \mathrm{GC}-\mathrm{qMS}$ and $\mathrm{GC} \times \mathrm{GC}-\mathrm{FID}$ were used as complementary techniques for the analysis of VOCs in air samples [109]. Over 125 VOCs from multiple classes were analyzed by using the method presented: alkanes, alkenes, aromatics, oxygenated hydrocarbons, and halocarbons. The group stressed the importance of optimizing the qMS data acquisition rate and demonstrated that qMS could provide valuable qualitative information, while FID is more accurate for quantitation. In a recent study, a miniaturized portable GC $\times$ GC $-\mu$ PID instrument was used for on-site monitoring of occupational exposure to volatile organic compounds in a paint shop [110]. The method was validated and compared to traditional VOC monitoring techniques. The $2 \mathrm{D}$ analysis resulted in enhanced peak capacity and shortened analysis time (10-15 min) as compared to those achieved by portable GC instruments.

Thousands of non-halogenated and halogenated compounds of anthropogenic origin are potentially present in environmental samples and the extra separating power of $\mathrm{GC} \times \mathrm{GC}$ is needed to resolve them. The examples reported in this review illustrated the advantages of using $\mathrm{GC} \times \mathrm{GC}$ for environmental analysis. Along with monitoring target analytes, GC×GC coupled with TOFMS, HRMS, and/or other MS detectors is urgently required in environmental analysis to detect and identify compounds that might be environmentally relevant but are not routinely analyzed. As there is no single analytical technique or method that can separate and detect all the contaminants present in environmental samples, complementary techniques such as LC or other hyphenated techniques [111] might be employed to fully characterize them.

The increased interest in $\mathrm{GC} \times \mathrm{GC}$ in the recent years proved that this technique is more accessible for environmental laboratories, yet not fully implemented as a routine analysis method in most cases. The required training of the analysts, the more time-consuming data processing, and the size of the resultant data files requiring powerful computers, could be what keeps most 
of the decision makers to step back from using this technique routinely. However, the simplified sample preparation techniques along with the increased separation power, accuracy and investigative capabilities of $\mathrm{GC} \times \mathrm{GC}$ might help overcome this drawback.

\subsection{Sccp priority substances}

\subsection{Halogenated organic contaminants in environmental samples}

The analysis of persistent halogenated organic pollutants in environmental samples is a challenge due to the very large number of compounds with varying chemical and physical properties.

Chlorinated paraffins (CPs) are complex mixtures of chlorinated n-alkanes with varying chain lengths $\left(\mathrm{C}_{10}\right.$ to $\left.\mathrm{C}_{30}\right)$ and degree of chlorination (30\% to $70 \%$ by weight). First introduced in 1930s, CPs are produced by the chlorination of $\mathrm{C}_{10}-\mathrm{C}_{30} \mathrm{n}$-alkane mixtures with molecular chlorine under forcing conditions. Commercial $\mathrm{CPs}$ are classified into three categories: short chain $\left(\mathrm{C}_{10^{-}}\right.$ $\left.\mathrm{C}_{13}\right)$, medium chain $\left(\mathrm{C}_{14}-\mathrm{C}_{17}\right)$, and long chain $\left(\geq \mathrm{C}_{18}\right)$, and further sub-categorized based on their percent weight content of chlorine: 40-50\%, 50-60\%, and 60-70\%. SCCPs $\left(\mathrm{C}_{10-13}\right)$ and MCCPs $\left(\mathrm{C}_{14-17}\right)$ are viscous, colourless or yellowish dense oils. The average chlorine content by weight is $30-52 \%$ for $\mathrm{C}_{18-20}$ liquid products, $40-54 \%$ for $\mathrm{C}_{>20}$ liquid products, and $70-72 \%$ for $\mathrm{C}_{>20}$ solid products [112-113]. Environmentally significant physical-chemical properties such as the vapor pressure (similar to other chlorinated organics like PCBs and toxaphenes) and octanol-water partition coefficient ( $\log \mathrm{K}_{\mathrm{ow}}$ from 4.4 to $>10$, very hydrophobic compounds) make these compounds persistent and bioaccumulative. They are classified as toxic for living organisms [112,114-115]. 
A 2003 survey of the uses and releases of CPs in Canada revealed that nearly all usage of short chain chlorinated paraffins (SCCPs) was in metalworking applications, with minor uses as flame retardants in plastics and rubber. Medium chain chlorinated paraffins (MCCPs) were mostly used in plastics and as lubrication additives, while long chain chlorinated paraffins (LCCPs) were mostly used as lubrication additives, as well as components of metalworking fluids and paints. Discharges into the environment can occur during production, transportation, use, storage and disposal of CPs [116]. These compounds were detected in many environmental matrices (sediment, soil, biota, air and waste) and are classified as priority toxic substances under Canada's Environmental Protection Act [21,117-118].

Due to the large number of congeners present in CP mixtures, these compounds were described as "the most challenging group of substance to analyse and quantify" [133]. Multi-step analytical methods including sample preparation, instrumental analysis and extensive quantification procedures are usually required to analyze these compounds. Recent reviews have summarized the sample preparation procedures, as well as the instrumental and quantification techniques used in different laboratories. One-dimensional chromatography coupled with different detectors is the most common technique employed for the analysis of CPs, viz. GC-ECD and GC-MS (NCILRMS and qTOF, NCI-HRMS, MS/MS,). Due to the extremely large number of different isomers and homologues, the chromatographic separation of $\mathrm{CP}$ mixtures into individual congeners is not achievable; consequently, different quantification methods were developed to minimize any bias in quantifying CPs [119-120]. More recently, $\mathrm{GC} \times \mathrm{GC}$ was also employed for the analysis of CPs

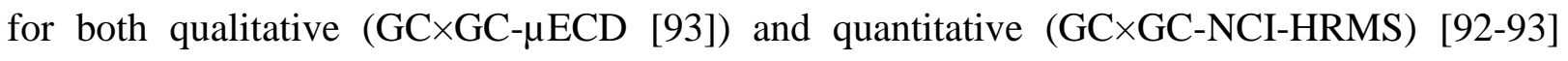
analyses. 
Mono-hydroxylated polychlorinated biphenyls (OH-PCB), a group of 837 possible congeners, are PCB metabolites produced by both biological and abiotic mechanisms [121-122]. Some of the formation pathways of OH-PCBs are through the oxidation of PCBs via cytochrome P450 (CYP) in biota [121,123-124], potential oxidation of PCBs in the gas phase by hydroxyl radicals $(\bullet \mathrm{OH})$ in natural environments $[121,125]$, transformation in waste water treatment plants [121] or degradation by microorganisms [126]. The empirical formula of the mono-hydroxylated-PCBs is $\mathrm{C}_{12} \mathrm{H}_{10-\mathrm{n}} \mathrm{Cl}_{\mathrm{n}} \mathrm{O}$, where $\mathrm{n}=1-10$ (Figure 1.15). Similar to their parent compounds, PCBs, the physical and chemical properties and the environmental behaviour of OH-PCBs is determined by the chlorination level and the pattern of chlorine and $-\mathrm{OH}$ substitution of the biphenyl: the higher the degree of chlorination, the higher the $\log \mathrm{K}_{\mathrm{ow}}$ value (from 4.5 to 9), the lower the Henry's constant and the lower the aqueous solubility. OH-PCBs are more water soluble than PCBs, thus, more bioavailable for the living organisms [121]. Hydroxylated PCBs have been studied and reported in humans [127-128] and different environmental matrices such as biota [129-130], sediments [131], sludges [132], air [133-134], water and precipitation. Toxicological effects of OH-PCBs were also studied for both humans and wildlife, showing that some OH-PCBs are more toxic than their parent compounds. Due to their similar structure with the thyroid hormones (thyroxine and triiodothyronine), these compounds showed binding affinity with the transport protein transthyretin (TTR) resulting in the disruption of the transport and metabolism of thyroid hormones and showing neurodevelopmental and behavioral effects [121]. 


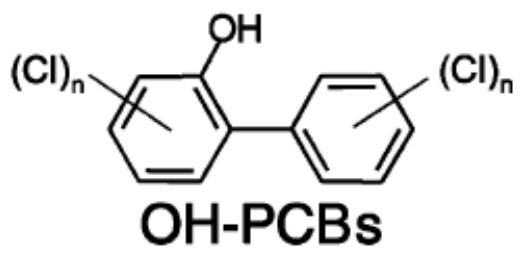

Figure 1.15 Structure of mono-hydroxylated PCBs (Reproduced from reference [135]).

The analysis of $\mathrm{OH}-\mathrm{PCB}$ s is very complex due to the high number of congeners present in this group, their physical-chemical properties, complex sample preparation and instrumental analysis methods, and the lack of availability of commercial standards (about 80 standards out of 837 possible congeners). Methods using gas chromatography coupled with different detectors such as ENCI-MS, MS/MS and HRMS were developed for the separation and quantitation of OH-PCB. When using GC-HRMS, a more sensitive analysis method and complex characterization of the OH-PCB isomers detected in the samples was possible $[125,136]$.

Polychlorinated diphenyl ethers (PCDE), a group of 209 possible isomers, have the empirical formula $\mathrm{C}_{12} \mathrm{H}_{10-n} \mathrm{Cl}_{n} \mathrm{O}$, where $\mathrm{n}=1-10$ (structural formula: Figure 1.16). These compounds showed properties very similar to those of polychlorinated biphenyls (PCBs), dibenzo-p-dioxins (PCDDs), dibenzofurans (PCDFs) and PCNs (polychlorinated naphthalenes), compound classes of significant environmental interest as they are ubiquitous in the environment, stable, can biomagnify and are known to be toxic to living organisms [137-138]. Their physical and chemical properties are not very well known; however, the calculated and predicted $\log \mathrm{K}_{\mathrm{ow}}$ values ranging from 4.45 to 8.16 , as well as aqueous solubility higher than that of PCBs, indicated that PCDEs are more bioavailable and have the potential for bioaccumulation [138]. PCDEs were 
identified as by-products of chlorinated phenols and chlorinated phenoxyacetic acids formulations [137-140], and have been found at wood waste sites, in fly ash and in municipal incinerator wastes [141-142]. PCDEs have been used as hydraulic fluids, electric insulators, flame retardants, lubricants and plasticizers, pesticides and synergistic pesticides [138-139]. These compounds have been reported in many environmental matrices such as biota and sediments; the studies have been summarized in several published reviews [137-140]. As their production has been much smaller than that of PCBs and production and sales decreased in the 1970s, it is believed that the widespread environmental occurrence of PCDEs is mainly due to their presence at high levels $(100-1000 \mathrm{mg} / \mathrm{kg})$ in the technical chlorophenols formulations [137].

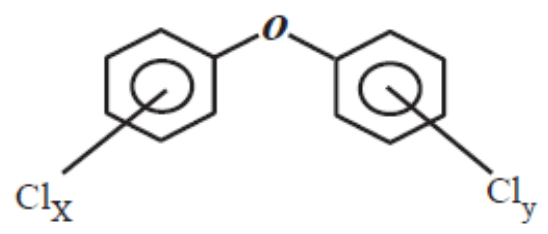

Figure 1.16 Chemical structure of PCDEs (Reproduced from reference [139]).

The analysis of PCDEs is very complex, as fractionation of the extracts is required prior to instrumental analysis to separate these compounds from other halogenated compound classes (e.g. PCDD and PCDFs) that are present in the samples at the same time. Analytical methods employing GC coupled with different detectors were developed for the analysis of PCDE; however, the lack of commercially available analytical standards makes the analytical procedures difficult. GC-ECD [143] was used in some studies, but GC coupled with both low resolution [144-146] and high resolution [147] MS operated in electron impact (EI) mode were the methods of choice. EI mass spectra of most PCDEs have an abundant molecular ion $\left(\mathrm{M}^{+}\right)$and a more 
abundant $\mathrm{M}^{++}-2 \mathrm{Cl}$ fragment that is mostly used in SIM analyses. Both external calibration, and, for more accuracy and precision, isotope dilution were used for quantification. The lack of commercially available labeled internal standards is the limiting factor for the development of isotope dilution methods [138].

\subsection{Scope of Thesis}

In the following chapters, the results of research on advances in the analysis of persistent organohalogen pollutants in environmental samples by using $\mathrm{GC} \times \mathrm{GC}$ are presented. The first research project's objective was to evaluate the performance of a new single-stage consumablefree thermal modulator developed at the University of Waterloo versus one of the industry's leading modulation system, the LECO quad-jet liquid-nitrogen $\left(\mathrm{LN}_{2}\right)$ modulator (LECO Corp., Benton Harbour, MI, USA). The chromatographic separations, as well as the performance data were directly compared to the routine accredited method used for the analysis of PCBs, OCs and CBs in solid sample. This accredited method was developed by me for the completion of the Masters of Applied Science and Management graduate program [28,76].

As most of the environmental laboratories perform targeted analysis for the routine testing of the "real-life" samples, many other environmentally significant persistent and/or emerging contaminants present in the samples are missed. The previously developed GC $\times$ GC method [28] has the potential to screen for other non-targeted halogenated contaminants at the same time with the target analysis. This method also reduces the number of sample preparation steps, improves data quality and eliminates fractionation of the extracts. Chapters 3,4 and 5 present the analysis of novel halogenated contaminants by $\mathrm{GC} \times \mathrm{GC}$ using both $\mu \mathrm{ECD}$ and TOFMS detectors. These compounds were initially identified in routinely analyzed environmental samples as non-target 
"unknown" compounds, and further identified as SCCPs (Chapter 3), OH-PCBs (Chapter 4) and PCDEs (Chapter 5). Analytical methods for qualitative and quantitative determinations were also developed. 


\section{Chapter 2}

\section{Evaluation of a Single-Stage Consumable-Free Modulator for GC $\times$ GC:}

\section{Analysis of Polychlorinated Biphenyls, Organochlorine Pesticides and}

\section{Chlorobenzenesii}

Analysis of persistent organic pollutants is a real challenge for environmental laboratories for many reasons, including a large number of compounds with varying chemical and physical properties present simultaneously in samples, concentration ranges varying from fg to $\mu \mathrm{g}$ levels for different compound classes, many isomers per contaminant class, etc. Very large amounts of numerous Stockholm priority organic pollutants (POPs) (approximately 1 million tons of PCBs and 2 million tons of DDT alone) [148] were manufactured during the last century and used in numerous industrial applications, as pesticides and flame retardants. Polychlorinated biphenyls $(\mathrm{PCBs})$, organochlorine pesticides (OCs) and chlorobenzenes (CBs) are routinely analyzed in a variety of sample matrices including fish, fatty food, and environmental samples typically using one dimensional GC. There is currently no single GC column phase that can separate all 209 PCBs or 210 polychlorinated dibenzo-p-dioxins/polychlorinated dibenzofurans (PCDD/Fs), and consequently at least 2 columns must be used for accurate quantification of these types of pollutants [149].

\footnotetext{
ii This chapter is based on the paper by A.M. Muscalu, M. Edwards, T. Górecki and E.J. Reiner, published as "Evaluation of a single-stage consumable-free modulator for comprehensive two-dimensional gas chromatography: Analysis of polychlorinated biphenyls, organochlorine pesticides and chlorobenzenes", J. Chromatogr. A 1391 (2015) 93-101
} 
First introduced in 1991, comprehensive two-dimensional gas chromatography (GC×GC) is a separation method offering increased peak capacity and selectivity relative to conventional onedimensional separation techniques such as traditional GC [1]. The technique requires that two GC columns of different selectivities be connected to each other through a modulating interface [10]. The modulator plays a crucial role in this method as it serves to sample primary column effluent, focus it into a narrow chromatographic band and reinject this band into the secondary column for further separation. In order for the technique to be truly comprehensive, any separation achieved in the primary column must be preserved in the second column, and complete transfer of effluent from the primary column to the secondary column must be accomplished [150-151]. Modulation must occur at precisely timed intervals throughout the analysis, typically between $\sim 2$ and $~ 10$ seconds. Considerable development in modulation technology has occurred over the last twenty years and has been summarized elsewhere [6]. Today, various $\mathrm{GC} \times \mathrm{GC}$ systems are commercially available from several vendors. These instruments operate using two primary techniques that can be classified as either flow modulation or thermal modulation [6]. Agilent offers a flow modulation device based upon their Capillary Flow Technology [15]. The high flow in the second dimension requires long secondary columns for adequate separation and makes the use of mass spectrometry detection with this technique difficult.

Thermal modulators operate on the principle of sampling, focusing and injecting primary column effluent into the second dimension column using alternatively activated hot and cold jets. The cold jets direct cooled nitrogen gas onto a segment of a column causing the analytes to be trapped in the stationary phase. A jet of hot air is then activated, remobilizing the trapped band into the second dimension column for further separation. LECO Corporation offers a popular dual-stage quad-jet system [152]. ZOEX Corporation offers a two-stage system using single cold 
and hot jets and a transfer line serving as a delay loop. Both manufactures also offer consumablefree systems; however, they showed inferior performance when compared to their cryogenic alternatives for volatile analytes.

$\mathrm{GC} \times \mathrm{GC}$ is gaining in popularity and in recent years the number of publications citing the use of this technique has grown exponentially [10]. Applications can be found in the fields of metabolomics [153-156], petroleum and petrochemicals [157-159], food, flavours and fragrances [160-162], and environmental analysis [28,61,163]. PCBs, OCs and CBs can be routinely analyzed using comprehensive two-dimensional gas chromatography for solid samples such as soil, sediment and sludge using the officially accredited $\mathrm{GC} \times \mathrm{GC}$ method developed at the Ontario Ministry of the Environment to reduce the number of sample preparation steps, improve data quality for these target classes of compounds and eliminate fractionation of the extracts, which is required with one-dimensional analysis [27-28]. This method has resulted in significant time and analysis costs savings. The $\mathrm{GC} \times \mathrm{GC}$ system yields excellent within- and between-class separations, and has replaced several conventional GC instruments. The GC×GC method developed has been shown previously to be both precise and accurate. The objective of this study was to evaluate the performance of a new single-stage consumable-free thermal modulator developed at the University of Waterloo (UW) versus the industry leading modulation system, the LECO (LECO Corp., Benton Harbour, MI, USA) quad-jet liquid-nitrogen ( $\left.\mathrm{LN}_{2}\right)$ modulator [152]. The chromatographic separations, as well as the performance data were directly compared to the routine accredited method used for the analysis of PCBs, OCs and CBs in solid samples [27-28]. 


\subsection{Experimental}

\subsubsection{Standards and samples}

PCB standards (BP-MS, BP-MS2, BP-MS3 - 82 compounds) were obtained from Wellington Laboratories (Guelph ON, Canada), while the chlorobenzenes (CB) and organochlorine pesticides (OC) standard mixtures, 15 and 23 compounds respectively, were acquired from Absolute Standards Inc. (Hamden, CT, USA). The multi-level calibration standard solutions containing $\mathrm{PCBc} / \mathrm{OC} / \mathrm{CB}$ were prepared in iso-octane to produce a calibration series with concentrations ranging from $1 \mathrm{ng} / \mathrm{mL}$ to $500 \mathrm{ng} / \mathrm{mL}$. 4,4'-Dibromo-octafluoro-biphenyl was added to each calibration solution at a concentration of $10 \mathrm{ng} / \mathrm{mL}$ as the internal standard for PCB congener quantification. Decachlorobiphenyl and 1,3,5-tribromobenzene (North Kingstown, RI, USA) were used as surrogates.

A portion of an air-dried soil/sediment/sludge sample was extracted using accelerated solvent extraction (ASE, Dionex ${ }^{\mathrm{TM}}$ ASE$^{\mathrm{TM}}$ 350, Thermo Scientific, Bannockburn IL, USA). The ASE conditions were as follows: one cycle extraction at $100{ }^{\circ} \mathrm{C}$, heat time $5 \mathrm{~min}$, , purge time 90 s, flush volume $60 \%$, extraction solvent $25 \%$ dichloromethane/75\% hexane (V/V). The resulting extract was then subjected to a single stage silica cartridge cleanup procedure prior to instrumental analysis. This simple and fast sample preparation method results in a significant reduction in sample handling and solvent requirements. No fractionation of extracts is required prior to instrumental analysis, which results in significant time- and analysis costs savings with definitive enhancement in data quality.

Sediment reference material SRM1944 (Standard Reference Material SRM1944 - New York/New Jersey Waterway Sediment) from National Institute of Standards and Technology (NIST - Gaithersburg, MD, USA) and another soil sample (CALA proficiency testing for PCB 
Total) were used to test the performance of the UW modulator. The "real" samples' test results obtained with the consumable-free modulator were compared to the performance data acquired with the industry standard $\mathrm{LN}_{2}$ modulator using the accredited routine method.

\subsubsection{GC×GC System}

The GC $\times$ GC $-\mu$ ECD system (LECO Corp., Benton Harbour, MI, USA) used for this study consisted of an Agilent 6890 gas chromatograph (Agilent Technologies, Santa Clara, CA, USA) equipped with a split/splitless injector and a $\mu \mathrm{ECD}$ detector. The following chromatographic column combination was used: $30 \mathrm{~m}, 0.25 \mathrm{~mm}$ i.d., $0.25 \mu \mathrm{m}$ film thickness DB-1 (100 \% dimethylpolysiloxane) from J\&W Scientific (Folsom, CA, USA) as the first dimension column, $1.6 \mathrm{~m}, 0.18 \mathrm{~mm}$ i.d., $0.18 \mu \mathrm{m}$ film thickness Rtx-PCB from Restek Corp. (Bellefonte, PA, U.S.A.) as the second dimension column, and a $0.3 \mathrm{~m}, 0.1 \mathrm{~mm}$ i.d. IP-Deact column (Restek Corp.) as the transfer line to the detector. The transfer line was used in the experiments only when the LECO modulator was used. One microliter samples were injected using the split/splitless injector operated in splitless mode at a temperature of $250{ }^{\circ} \mathrm{C}$ while using a $4 \mathrm{~mm}$ i.d. gooseneck liner (Restek Corp.). Helium gas with a purity of $99.999 \%$ was used as carrier gas at $1.2 \mathrm{~mL} / \mathrm{min}$ flow rate. The micro-ECD system was operated at $300{ }^{\circ} \mathrm{C}$ using data acquisition rate of $50 \mathrm{~Hz}$, with 5 $\%$ methane in argon as the detector make-up gas at a flow-rate of $150 \mathrm{~mL} / \mathrm{min}$. ChromaTof software (LECO Corp.) was used for data acquisition and processing for all analyses performed for this study.

\subsubsection{Modulation}

The LECO $\mathrm{LN}_{2}$ quad-jet dual-stage modulator initially installed on the $\mathrm{GC} \times \mathrm{GC}$ system was used to develop the routine method for the analysis of PCBs and other halogenated compounds. Details are presented elsewhere [28]. The UW modulator prototype was installed on the same 
system in order to obtain directly comparable data. This single-stage thermal modulator for GC $\times$ GC required no consumables for operation and was developed to trap, focus and re-inject the analytes into the second column using a specially prepared coated stainless steel capillary trap (Figure 2.1). Focusing of the analytes within this trap was assisted through compression of the trap between two ceramic cooling blocks. Injection of focused chromatographic bands into the secondary column was accomplished using a capacitive discharge power supply to resistively heat the trap, while rapid cooling was accomplished by compressing the steel capillary between two passively or actively cooled ceramic pads.

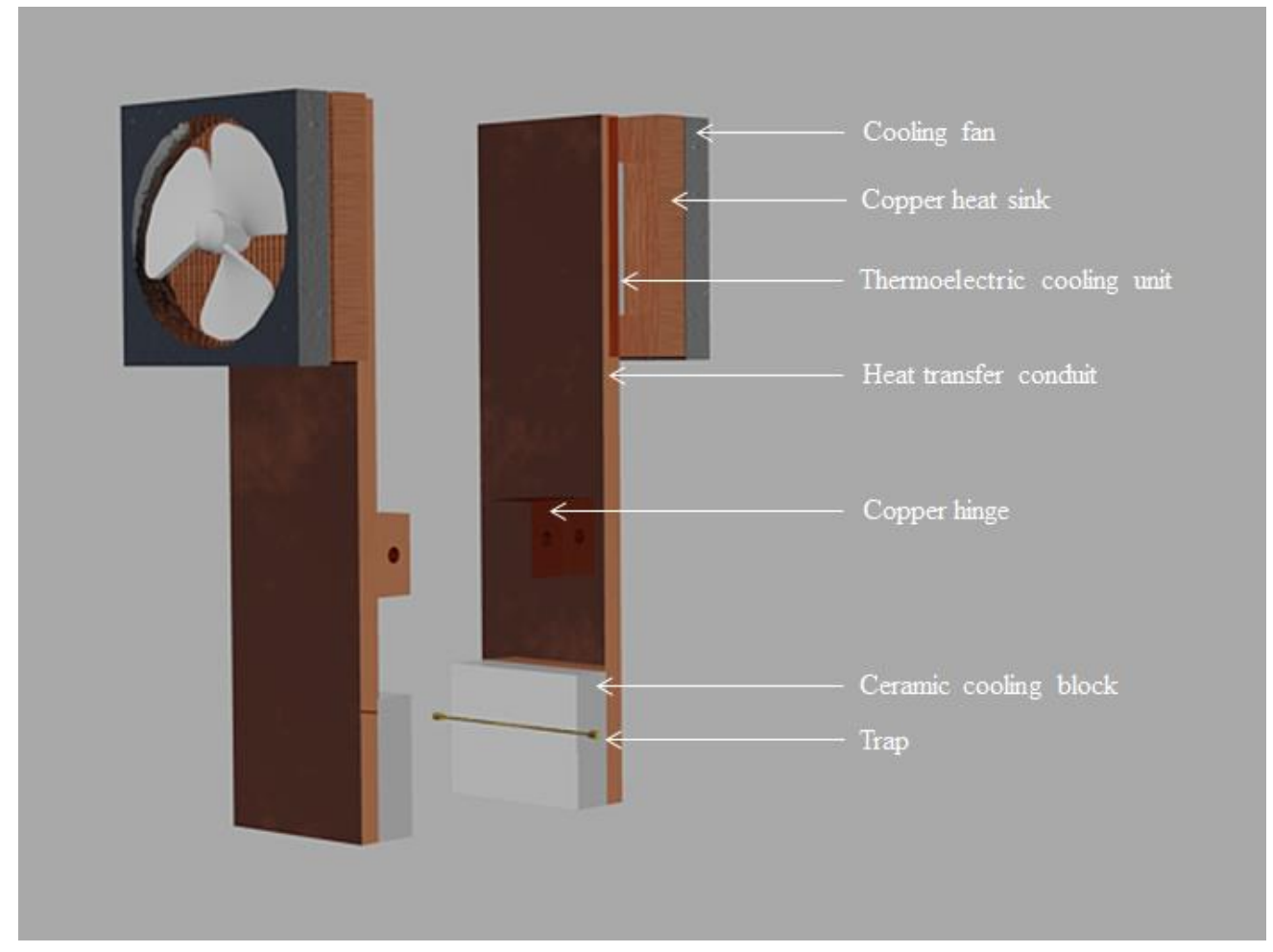

Figure 2.1 Single-stage, consumable-free thermal modulation system.

Once all the columns/parts were installed and leak-checked, the column flow was measured at the secondary column outlet and set to $1.2 \mathrm{~mL} / \mathrm{min}$. The outlet was then connected to the $\mu \mathrm{ECD}$. 
The timing of the modulation with the GC run was critical for data acquisition and to generate reproducible $\mathrm{GC} \times \mathrm{GC}$ chromatograms. For this purpose, the timing of the modulation was synchronized with the data acquisition clock of the FID electrometer, installed on the system but not used for the analysis purposes. The modulation period set for all the runs was $4 \mathrm{~s}$, similar to the quad-jet, dual-stage modulator. The secondary oven was not used for any experiments involving the UW modulator.

\subsection{Results and Discussion}

\subsubsection{GC×GC Separations}

The evaluation of the chromatographic separations was first carried out using the consumable-free modulator with passively cooled ceramic plates. When this type of cooling was employed, breakthrough was observed for some analytes as the ceramic plates were not cooling efficiently enough when the GC oven was heating during the run and cooling down in-between analytical runs (Figure 2.2A). Higher voltage was tested with the trap, but it had a deleterious effect on trap longevity making the system impractical for routine use (Figure 2.2B). To improve the modulator's performance, an active cooling system using thermoelectric coolers attached to the heat conduits to which the ceramic plates were mounted was installed, and the breakthrough was significantly reduced for most problem compounds (Figure 2.2C). This set-up was used for all performance data presented in this paper. Two exceptions were observed for the more volatile non-aromatic compounds: hexachloro-ethane (HCE) and hexachloro-butadiene (HCBD). Different experiments were performed to minimize the breakthrough of these compounds and the best results were obtained when the oven temperature was programmed to start at $60{ }^{\circ} \mathrm{C}$ instead of the $80{ }^{\circ} \mathrm{C}$ used with the validated method. The outcome of the routine $\mathrm{GC} \times \mathrm{GC}$ analysis for $\mathrm{PCBs} / \mathrm{OCs} / \mathrm{CBs}$ when using the LECO system is presented in Figure 2.2D showing that PCB 
congeners were separated according to their degree of chlorination, as well as their planar structure (more retained in the ${ }^{2} \mathrm{D}$ plane). Wrap-around was observed for both systems, but did not affect the separation of any of the target compounds. 

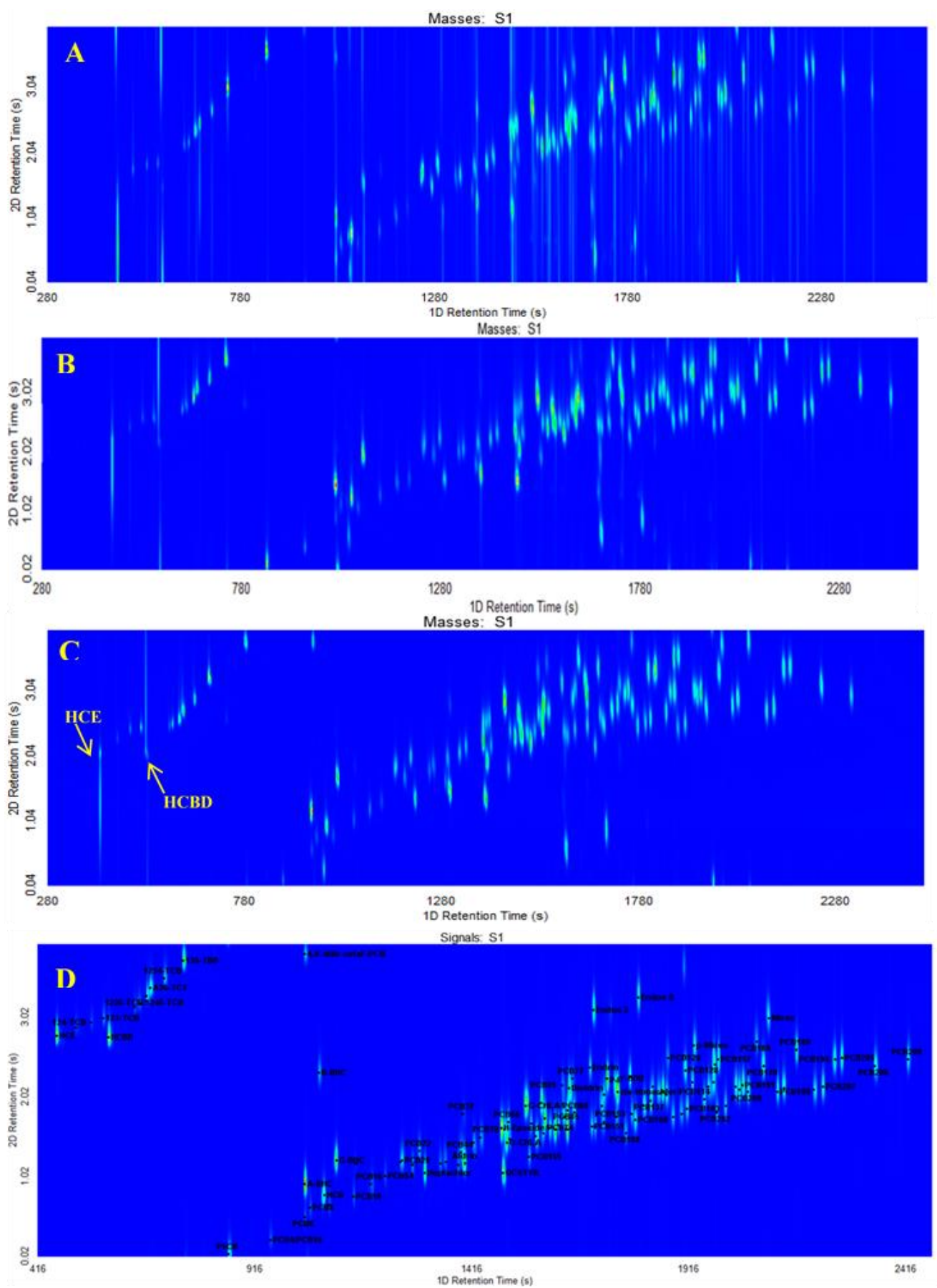

Figure 2.2 GC x GC- $\mu$ ECD contour plot of PCB/OC/CB standard mix (118 target compounds). Analysis was performed with: (A) consumable-free modulator with passive cooling for the ceramic plates, $22.4 \mathrm{~V}$; (B) consumable-free modulator with passive cooling for the ceramic plates at higher voltage, $24.4 \mathrm{~V}$; (C) consumable-free modulator with active cooling for the ceramic plates, $22.8 \mathrm{~V}$; (D) $\mathrm{LN}_{2}$ modulator 
The GC $\times$ GC- $\mu \mathrm{ECD}$ routine method using the cryogenic modulator and targeting 118 compounds showed a total of eight co-elutions: 3 within-class (PCB4/PCB10, PCB90/PCB101, 1,2,3,5-tetrachlorobenzene (1,2,3,5-TCB)/1,2,4,5- tetrachlorobenzene $(1,2,4,5-\mathrm{TCB})$ and 4 between-class (heptachlor-epoxide/PCB74, gamma-chlordane $(\gamma$-CHLA)/PCB60, cisnonachlor/PCB114, and methoxychlor (DMDT)/ PCB171). Figures 2.3A and B indicate that, without further optimization of the GC method when using the single stage thermal modulator, two of these co-elutions were resolved: heptachlor-epoxide/PCB74 and cis-nonachlor/PCB114, which was further confirmed by analyzing individual standards for each of the compounds. These separations were very important as PCB74 is one of the major congeners in Aroclor 1232, 1242 and 1248, and PCB114 is one of the twelve dioxin-like PCBs. On the other hand, two compounds that were separated with the quad-jet modulator were co-eluting with the UW modulator tested: PCB99 (large contributor in Aroclors) and alpha-chlordane (ubiquitous in environmental samples). To assess if this co-elution was due to the modulator's performance and not to the slight difference in the column set-up, the experiments with the quad-jet modulator were repeated with the $30 \mathrm{~cm}$ transfer line to the detector removed. The experiments showed that these compounds also co-eluted with the quad-jet modulator when the transfer line was not used, hence it was concluded that both systems performed similarly under comparable conditions. 

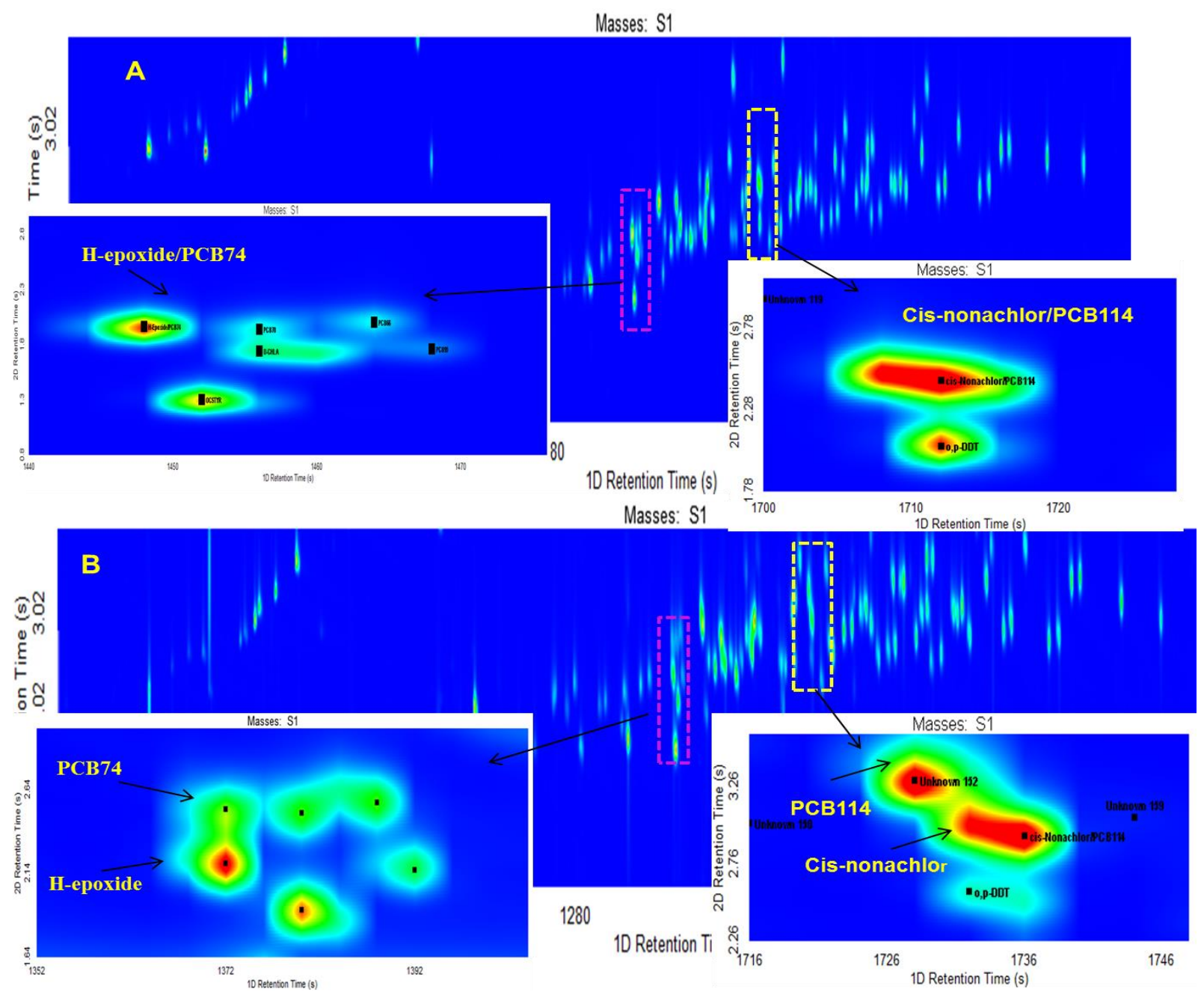

Figure 2.3 GC x GC- $\mu$ ECD contour plot of (A) co-elutions when using the quad-jet modulator and (B) resolving co-elutions when using the new consumable-free thermal modulator.

\subsubsection{Peak Characteristics}

The peak widths in GC $\times \mathrm{GC}$ are very narrow due to the modulation process: the first column eluent, trapped in small fractions by thermal focusing during the modulation cycle, is re-injected into the second dimension column resulting in very fast separation in the second dimension (peak width $\sim 50-\sim 600 \mathrm{~ms})$. To evaluate the modulation's efficiency, the peak widths obtained with the single-stage thermal modulator were compared with the peak widths data from the accredited 
method. Table 2.1 and Figure 2.4 present the peak attributes, such as peak widths and heights, for selected target compounds for ten replicates analyzed with each system (for the complete set of data for the target analytes see Appendix B, Tables B.1 and B.2) as obtained with both modulation types for a standard mix of $20 \mathrm{ng} / \mathrm{mL}$. The data showed that peak widths at half height ranged from 100 to $250 \mathrm{~ms}$ for the $\mathrm{LN}_{2}$ system and from 150 to $320 \mathrm{~ms}$ for the thermal modulator. When peak heights were compared, both systems produced similar results (Figure 2.4).

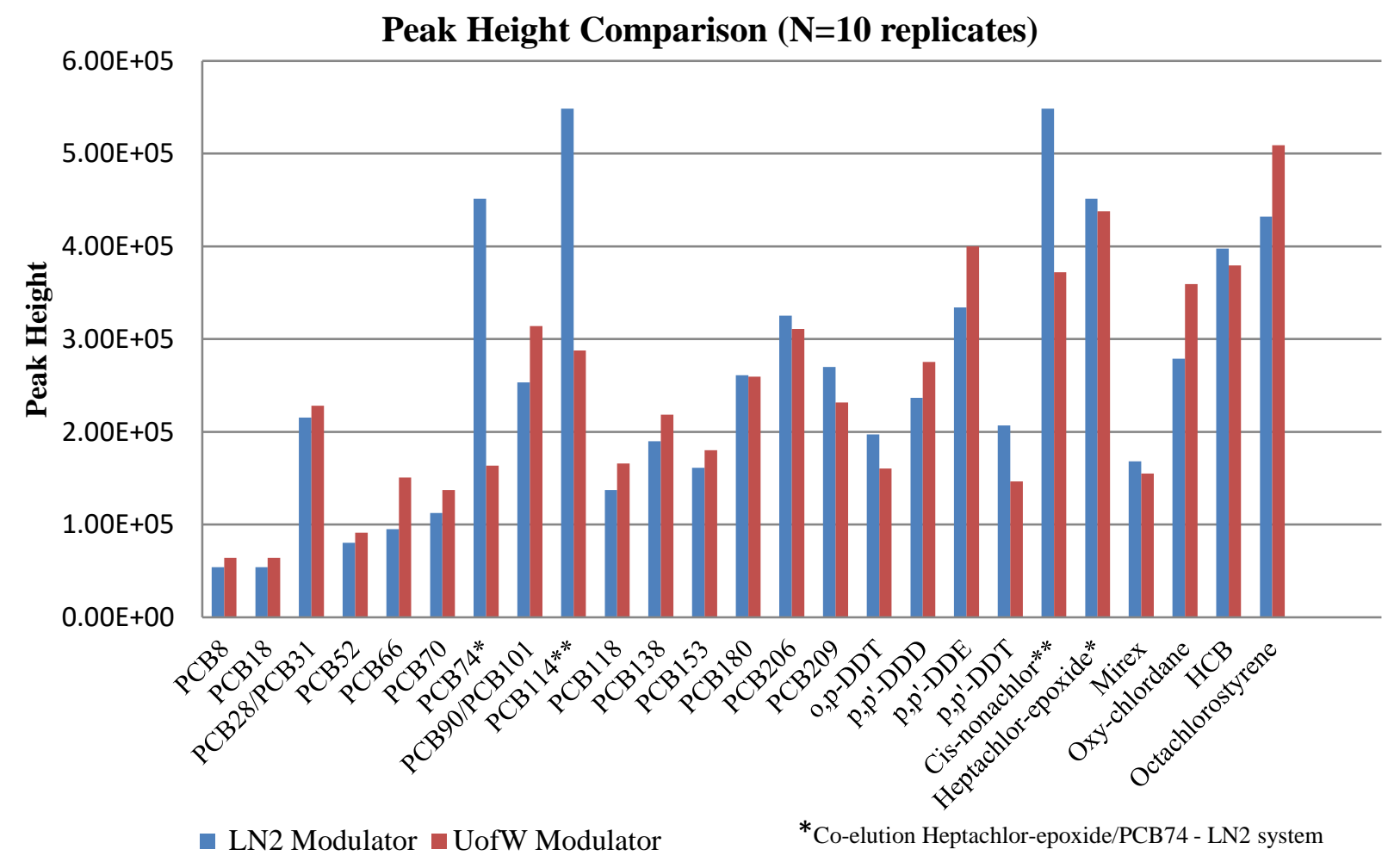

Figure 2.4 Peak height comparison for selected compounds analyzed with the $\mathrm{LN}_{2}$ modulator and the consumable-free thermal modulator. 
Table 2.1 Peak width attributes: Full Width at Half Height (s) as obtained with both modulation systems

\begin{tabular}{|l|c|c|}
\hline \multirow{2}{*}{ Name } & \multicolumn{2}{|c|}{ Full Width at Half Height (s; N=10) } \\
\cline { 2 - 3 } & LN2 Modulator & $\begin{array}{c}\text { Consumable-free } \\
\text { Modulator }\end{array}$ \\
\hline PCB8 & 0.169 & 0.215 \\
\hline PCB18 & 0.172 & 0.223 \\
\hline PCB28/PCB31 & 0.189 & 0.268 \\
\hline PCB52 & 0.182 & 0.246 \\
\hline PCB66 & 0.209 & 0.273 \\
\hline PCB70 & 0.182 & 0.279 \\
\hline PCB74* & 0.195 & 0.249 \\
\hline PCB90/PCB101 & 0.191 & 0.254 \\
\hline PCB114** & 0.205 & 0.322 \\
\hline PCB118 & 0.206 & 0.288 \\
\hline PCB138 & 0.203 & 0.276 \\
\hline PCB153 & 0.199 & 0.269 \\
\hline PCB180 & 0.205 & 0.276 \\
\hline PCB206 & 0.218 & 0.288 \\
\hline PCB209 & 0.210 & 0.288 \\
\hline o,p-DDT & 0.196 & 0.289 \\
\hline p,p'-DDD & 0.206 & 0.290 \\
\hline p,p'-DDE & 0.233 & 0.284 \\
\hline p,p'-DDT & 0.208 & 0.285 \\
\hline Cis-nonachlor** & 0.205 & 0.295 \\
\hline Heptachlor-epoxide* & 0.195 & 0.295 \\
\hline Mirex & 0.239 & 0.317 \\
\hline Oxy-chlordane & 0.213 & 0.241 \\
\hline HCB & 0.163 & 0.204 \\
\hline Octachlorostyrene & 0.172 & 0.220 \\
\hline & & \\
\hline
\end{tabular}

*Co-elution Heptachlor-epoxide/PCB74 - LN2 system

**Co-elution Cis-nonachlor/PCB114 - LN2 system

\subsubsection{Thermal Modulator Performance: Quantification, Repeatability and Reproducibility}

$\mathrm{GC} \times \mathrm{GC}$ calibration was performed using the $\mathrm{PCB} / \mathrm{OC} / \mathrm{CB}$ standard mixture at six different concentration levels ranging from 1 to $500 \mathrm{ng} / \mathrm{mL}$ to develop second order calibration curves used 
for quantification. External standard calibration method was used for the $\mathrm{OC}$ and $\mathrm{CB}$, and internal standard calibration procedure was used for PCB quantification. Retention reference compounds and the internal standard were used to check retention time stability between runs. An initial assessment of the modulator was performed by evaluating the instrument detection limits (IDL) for each of the compounds. Five replicates of the low level standards $(1 \mathrm{ng} / \mathrm{mL})$ were analysed for IDL calculations. When using the consumable-free modulator, the IDLs ranged from 0.1 to $0.4 \mathrm{ng} / \mathrm{mL}$, similar to the data obtained with the $\mathrm{LN}_{2}$ modulator. Beside the IDLs, the data presented in Table 2.2 for selected compounds (see Appendix B, Table B.3 for the complete list of targets) showed that the instrument precision at the low level $(1 \mathrm{ng} / \mathrm{mL})$ expressed as relative standard deviation (\% RSD) ranged from 1 to $14 \%$ for PCBs, 1 to $4 \%$ for CBs, and 1 to $9 \%$ for OCs (with DDTs and heptachlor poorer performers, at $17 \%$ and $31 \%$ respectively). 
Table 2.2 Instrument Detection Limits (ng/mL) for the GC $\times \mathrm{GC}-\mu \mathrm{ECD}$ systems with the two modulators

\begin{tabular}{|c|c|c|c|c|c|c|c|}
\hline \multirow{2}{*}{ Name } & \multirow{2}{*}{$\begin{array}{c}\text { Design } \\
(\mathrm{ng} / \mathrm{mL})\end{array}$} & \multicolumn{3}{|c|}{$\begin{array}{l}\mathrm{LN}_{2} \text { Commercial Modulator } \\
(\mathrm{N}=9)\end{array}$} & \multicolumn{3}{|c|}{$\begin{array}{c}\text { Consumable-free Modulator } \\
(\mathrm{N}=5)\end{array}$} \\
\hline & & Avg. & $\%$ RSD & IDL & Avg. & $\%$ RSD & IDL \\
\hline PCB8 & 1 & 0.95 & 5.77 & 0.16 & 0.81 & 11.33 & 0.28 \\
\hline PCB18 & 1 & 1.05 & 1.99 & 0.06 & 0.89 & 5.15 & 0.14 \\
\hline PCB28/РCB31 & 2 & 1.78 & 1.08 & 0.08 & 2.07 & 3.26 & 0.20 \\
\hline PCB52 & 1 & 0.94 & 12.50 & 0.35 & 1.04 & 6.56 & 0.20 \\
\hline PCB66 & 1 & 0.98 & 3.82 & 0.11 & 1.01 & 4.39 & 0.13 \\
\hline PCB70 & 1 & 0.98 & 7.17 & 0.21 & 0.96 & 5.37 & 0.16 \\
\hline PCB74* & $2(1)$ & 1.96 & 1.81 & 0.10 & 1.17 & 1.15 & 0.04 \\
\hline PCB90/PCB101 & 2 & 2.04 & 5.40 & 0.33 & 2.12 & 1.31 & 0.08 \\
\hline PCB114** & $2(1)$ & 1.89 & 3.26 & 0.17 & 0.12 & 0.35 & 0.35 \\
\hline PCB118 & 1 & 1.07 & 3.02 & 0.10 & 1.21 & 2.80 & 0.10 \\
\hline PCB138 & 1 & 1.12 & 2.36 & 0.08 & 0.93 & 4.30 & 0.12 \\
\hline PCB153 & 1 & 1.00 & 4.08 & 0.12 & 0.89 & 4.63 & 0.12 \\
\hline PCB180 & 1 & 1.07 & 3.67 & 0.12 & 0.94 & 2.76 & 0.08 \\
\hline PCB206 & 1 & 1.02 & 11.02 & 0.34 & 1.00 & 3.32 & 0.10 \\
\hline PCB209 & 1 & 1.05 & 2.06 & 0.07 & 1.13 & 1.45 & 0.05 \\
\hline $\mathrm{o}, \mathrm{p}-\mathrm{DDT}$ & 1 & 1.31 & 4.02 & 0.12 & 0.99 & 17.05 & 0.51 \\
\hline p,p'-DDD & 1 & 0.87 & 10.76 & 0.42 & 1.17 & 2.04 & 0.07 \\
\hline $\mathrm{p}, \mathrm{p}$-DDE & 1 & 1.02 & 4.17 & 0.11 & 1.23 & 2.42 & 0.09 \\
\hline p,p'-DDT & 1 & 1.40 & 2.43 & 0.07 & 1.17 & 8.74 & 0.31 \\
\hline Cis-nonachlor** & 1 & 1.89 & 3.26 & 0.17 & 1.02 & 12.09 & 0.37 \\
\hline Heptachlor-epoxide* & 1 & 1.96 & 1.81 & 0.10 & 1.19 & 2.40 & 0.09 \\
\hline Mirex & 1 & 0.82 & 3.50 & 0.15 & 1.08 & 8.39 & 0.27 \\
\hline Oxy-chlordane & 1 & 1.03 & 2.05 & 0.07 & 1.05 & 7.65 & 0.24 \\
\hline $\mathrm{HCB}$ & 1 & 0.83 & 3.24 & 0.08 & 1.11 & 1.76 & 0.06 \\
\hline Octachlorostyrene & 1 & 0.86 & 2.03 & 0.05 & 1.15 & 3.10 & 0.11 \\
\hline
\end{tabular}

*Co-elution Heptachlor-epoxide/PCB74 - LN2 system

**Co-elution Cis-nonachlor/PCB114 - LN2 system

To assess the within-day quantitation repeatability, ten replicates of the $20 \mathrm{ng} / \mathrm{mL}$ standard were analyzed and the data is presented in Table 2.3 (see Appendix B, Table B.4 for the complete list of targets). Experimental results showed \% RSDs ranging from 2 to $11 \%$ for PCBs (except 
PCB114-21\% and PCB85-13\%; poor performance, not baseline resolved), 4 to $8 \%$ for CBs and 3 to $16 \%$ for OCs (except endrin, $24 \%$ ), very similar to the data obtained with the $\mathrm{LN}_{2}$ modulator.

To further evaluate the instrument performance, between-run results of 5 replicates at 20 $\mathrm{ng} / \mathrm{mL}$ were evaluated and the data showed \% RSDs between 3 and $15 \%$ for PCBs, 3 to $13 \%$ for OCs (exceptions included endrin at $33 \%$, p,p'-DDT at $45 \%$ and heptachlor at $51 \%$ ), and 6 to $12 \%$ for CBs (see Appendix B, Table B.5 for all analytes).

Table 2.3 Repeatability - within-run standards at $20 \mathrm{ng} / \mathrm{mL}$ : PCBs with different chlorination level, OCs and CBs

\begin{tabular}{|c|c|c|c|c|c|c|}
\hline \multirow{2}{*}{\multicolumn{2}{|c|}{ Compound Name }} & \multirow{3}{*}{$\begin{array}{c}\text { Expected } \\
\text { amount } \\
(\mathrm{ng} / \mathrm{mL})\end{array}$} & \multicolumn{2}{|c|}{$\begin{array}{c}\mathrm{LN}_{2} \text { Modulator } \\
(\mathrm{N}=10)\end{array}$} & \multicolumn{2}{|c|}{$\begin{array}{l}\text { Consumable-free } \\
\text { Modulator }(\mathrm{N}=10)\end{array}$} \\
\hline & & & \multirow{2}{*}{ Average } & \multirow{2}{*}{$\begin{array}{c}\text { RSD } \\
(\%)\end{array}$} & \multirow{2}{*}{ Average } & \multirow{2}{*}{$\begin{array}{l}\text { RSD } \\
(\%)\end{array}$} \\
\hline EU Indicator PCBs & \# Chlorines & & & & & \\
\hline PCB28/PCB31 & $\begin{array}{l}2,4,4^{\prime}-/ 2,4 ', 5- \\
\text { Trichlorobiphenyl }\end{array}$ & $\begin{array}{c}40 \\
\text { (coelution) }\end{array}$ & 41.8 & 3.8 & 38.8 & 2.6 \\
\hline PCB52 & $\begin{array}{l}2,2 ', 5,5^{\prime}- \\
\text { Tetrachlorobiphenyl }\end{array}$ & 20 & 20.5 & 0.7 & 20.1 & 3.4 \\
\hline PCB90/PCB101 & $\begin{array}{l}2,2^{\prime}, 3,4^{\prime}, 5-/ 2,2^{\prime}, 4,5,5^{\prime}- \\
\text { Pentachlorobiphenyl }\end{array}$ & $\begin{array}{c}40 \\
\text { (coelution) }\end{array}$ & 43.2 & 1.2 & 38.2 & 2.6 \\
\hline PCB118 & $\begin{array}{l}\text { 2,3',4,4',5- } \\
\text { Pentachlorobiphenyl }\end{array}$ & 20 & 18.0 & 5.8 & 21.1 & 5.3 \\
\hline PCB138 & $\begin{array}{l}2,2^{\prime}, 3,4,44^{\prime}, 5^{\prime}- \\
\text { Hexachlorobiphenyl }\end{array}$ & 20 & 20.2 & 2.9 & 18.8 & 4.8 \\
\hline PCB 153 & $\begin{array}{l}2,2^{\prime}, 4,4^{\prime}, 5,5^{\prime}- \\
\text { Hexachlorobiphenyl }\end{array}$ & 20 & 22.8 & 7.8 & 18.8 & 12.9 \\
\hline PCB180 & $\begin{array}{l}\text { 2,2',3,4,4',5,5'- } \\
\text { Heptachlorobiphenyl }\end{array}$ & 20 & 21.7 & 4.6 & 19.0 & 6.9 \\
\hline Other PCBs & \# Chlorines & & & & & \\
\hline PCB8 & 2,4'-Dichlorobiphenyl & 20 & 21.2 & 2.7 & 20.3 & 3.1 \\
\hline PCB 18 & \begin{tabular}{|l|}
$2,2 ', 5-$ \\
Trichlorobiphenyl \\
\end{tabular} & 20 & 20.4 & 2.9 & 21.1 & 2.3 \\
\hline PCB206 & $\begin{array}{l}\text { 2,2',3,3',4,4',5,5',6- } \\
\text { Nonachlorobiphenyl }\end{array}$ & 20 & 21.8 & 7.9 & 18.8 & 3.1 \\
\hline РCB209* & $\begin{array}{l}2,2^{\prime}, 3,3^{\prime}, 4,44^{\prime}, 5,5^{\prime}, 6,6^{\prime}- \\
\text { Decachlorobiphenyl }\end{array}$ & 20 & 20.7 & 6.9 & 18.7 & 4.2 \\
\hline
\end{tabular}


Table 2.3 Repeatability - within-run standards at $20 \mathrm{ng} / \mathrm{mL}$ : PCBs with different chlorination level, OCs and CBs

\begin{tabular}{|c|c|c|c|c|c|c|}
\hline \multirow{2}{*}{\multicolumn{2}{|c|}{ Compound Name }} & \multirow{3}{*}{$\begin{array}{c}\text { Expected } \\
\text { amount } \\
(\mathrm{ng} / \mathrm{mL})\end{array}$} & \multicolumn{2}{|c|}{$\begin{array}{c}\mathbf{L N}_{2} \text { Modulator } \\
(\mathrm{N}=10)\end{array}$} & \multicolumn{2}{|c|}{$\begin{array}{c}\text { Consumable-free } \\
\text { Modulator }(\mathrm{N}=10)\end{array}$} \\
\hline & & & \multirow{2}{*}{ Average } & \multirow{2}{*}{$\begin{array}{l}\text { RSD } \\
(\%)\end{array}$} & \multirow{2}{*}{ Average } & \multirow{2}{*}{$\begin{array}{l}\text { RSD } \\
(\%)\end{array}$} \\
\hline EU Indicator PCBs & \# Chlorines & & & & & \\
\hline \multicolumn{7}{|c|}{ OCs } \\
\hline \multicolumn{2}{|c|}{$\mathrm{p}, \mathrm{p}^{\prime}-\mathrm{DDE}$} & 20 & 18.8 & 3.3 & 18.2 & 4.7 \\
\hline \multicolumn{2}{|c|}{ Mirex } & 20 & 18.1 & 7.7 & 18.5 & 2.3 \\
\hline \multicolumn{2}{|c|}{ Oxy-chlordane } & 20 & 19.1 & 7.9 & 19.7 & 6.5 \\
\hline \multicolumn{2}{|c|}{ Heptachlor-epoxide** } & 20 & N/A & N/A & 20.8 & 11.5 \\
\hline \multicolumn{7}{|c|}{ CBs } \\
\hline \multicolumn{2}{|c|}{$\mathrm{HCB}$} & 20 & 22.3 & 3.4 & 19.8 & 5.8 \\
\hline \multicolumn{2}{|c|}{ Octachlorostyrene } & 20 & 20.0 & 7.9 & 19.5 & 6.2 \\
\hline \multicolumn{2}{|c|}{ 1,3,5-tribromobenzene* } & 20 & 21.9 & 4.4 & 20.7 & 5.0 \\
\hline
\end{tabular}

*Surrogates

**Co-elutes with PCB74

SRM1944, a NIST sediment standard reference sample, was one of the reference materials used to determine the efficiency of the extraction, clean-up and instrumental procedures, and to assess the accuracy of the GC $\times$ GC- $\mu$ ECD method prior to accreditation. One of these SRM1944 extracts, previously analyzed with the $\mathrm{LN}_{2}$ system, was re-injected into the system using the new consumable-free modulator and the recoveries of the target analytes were determined. These analyses assessed the performance of the modulators when analyzing real samples where numerous interferences were present. The results presented in Figure 2.5A showed that the performance of both modulators was comparable. The error bars represent the standard deviations for the SRM1944 certified values. The target compounds were separated in the second dimension from the other classes of contaminants present, similar to the routine method's separation (Figure 2.5B). In addition to accurately quantifying the target compounds, the analyses performed with 
the UW modulator showed similar enhanced separations of the targets from matrix interferences and other classes of contaminants present in the sediment samples.

Figure $2.5 \mathrm{~A}$

Sediment Reference Material

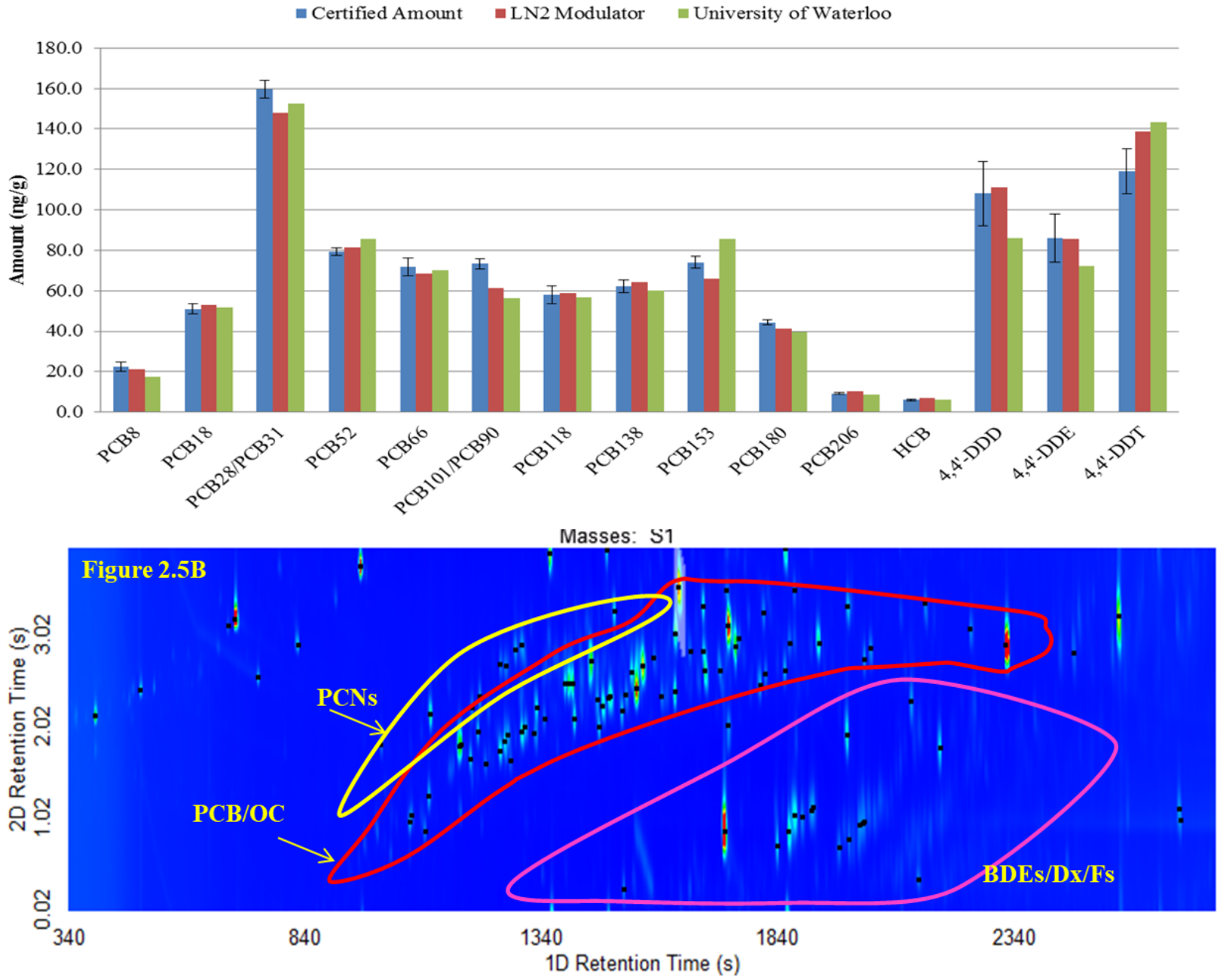

Figure 2.5 Sediment Reference Material (SRM1944) Comparison: (A) Certified values comparison as obtained from both systems; (B) Two-dimensional chromatograms showing the target compounds' separation from different interferences present in the sediment sample when using the consumable-free single stage modulator. (BDE - brominated diphenylether, Dx polychlorinated dioxins, Fs - polychlorinated furans) 
The performance of the UW modulator was also evaluated by analyzing a proficiency testing soil sample for PCBs known to have many interferences present, and the final result expressed as PCB total (sum of all the PCBc with concentrations above method's detection limits) was compared to the data obtained from the routine method as well as the design value. The quantitative results were very comparable between the tests: $77.9 \mu \mathrm{g} / \mathrm{g}$ for the consumable-free modulator, $85.4 \mu \mathrm{g} / \mathrm{g}$ for the routine method compared to the design value of $99.36 \mu \mathrm{g} / \mathrm{g}$.

\subsubsection{Robustness - between-run and between-trap system reproducibility}

To test the system's robustness and assess its suitability for routine analysis, we evaluated the ${ }^{1} \mathrm{D}$ and ${ }^{2} \mathrm{D}$ retention time shifts in-between analytical runs. The results were very encouraging as negligible shifts in retention times were observed for both within-day and between-day comparisons of the studied samples. For instance, the \% RSD of the within-run retention time shifts calculated for the routine method's five reference compounds (1,3,5-tribromobenzene, $p, p$ 'DDE, PCB52, PCB151 and PCB199), for ten replicates of the $20 \mathrm{ng} / \mathrm{mL}$ standard, ranged from 0.3 to $0.6 \%$ in the first dimension and from 0.8 to $2 \% \mathrm{~s}$ in the $2^{\text {nd }}$ dimension.

Beside the retention time shifts, it was very important to have reproducible chromatograms when the trap needed to be replaced. Changing the column set for the routine method (DB-1 x Rtx-PCB x transfer line) required very meticulous adjustments: it was critical to have a specific length of the ${ }^{2} \mathrm{D}$ column placed in the main oven upstream of the modulator and an exact length of the transfer line to the detector to obtain reproducible chromatograms. The consumable-free modulator was not using the secondary oven and the column lengths in the ${ }^{2} \mathrm{D}$ oven or transfer line were not variables in this case. For the tests, the trap used for the determination of the performance data presented in this paper was switched for a new trap and tested by injecting three replicates of the calibration standard at $20 \mathrm{ng} / \mathrm{mL}$. The retention times of the five reference 
compounds used to monitor the time shifts for the routine method (1,3,5-tribromobenzene, $\mathrm{p}, \mathrm{p}$ DDE, PCB52, PCB151 and PCB199) were checked for the old and the new trap and negligible shifts were observed. All the retention time shifts fell in-between the ranges observed for the within-run retention time \% RSDs presented above.

\subsection{Conclusions}

Comprehensive two-dimensional gas chromatography coupled with a micro electron capture detector has been used as a sensitive and selective routine method for the determination of PCBs, OCs, and CBs in sediments, soils and sludges in a single analytical run for the last five years in the MOECC laboratory. The method proved to be an excellent analytical tool in the assessment of the presence of other additional compounds and the initial identification and monitoring of new and emerging halogenated compounds present in sample extracts. The evaluation of the new consumable-free single stage thermal modulator was performed for the same target compounds as the accredited method and focused on the chromatographic separations and system's repeatability and reproducibility of the results. The performance of the consumable-free thermal modulation system proved to be comparable to the commercially available $\mathrm{LN}_{2}$ modulator. The experimental data obtained was accurate and precise for the standards and reference material tested. Also, without any further optimization of the GC method and without using the secondary oven, two co-elutions found in the $\mathrm{LN}_{2}$ method were resolved when the newly developed modulator was used. This very simple, consumable free and cost effective modulator was very easy to install and operate on any GC system making it a less expensive alternative for performing GC $\times \mathrm{GC}$ separations. Furthermore, its flexibility gives new opportunities of performing comprehensive two-dimensional GC with other detectors that are fast enough to handle the narrow peaks generated by GC×GC. 


\section{Chapter 3}

\section{The Quantification of Short Chain Chlorinated Paraffins in Sediment Samples Using Comprehensive Two-Dimensional Gas Chromatography with $\mu E C D$ Detection ${ }^{\mathrm{iii}}$}

First produced in the 1930s, chlorinated paraffins (CPs) are complex mixtures of chlorinated n-alkanes with different carbon chain lengths $\left(\mathrm{C}_{10}\right.$ to $\left.\mathrm{C}_{30}\right)$ and degree of chlorination (30\% to $70 \%$ by weight). They are produced by the chlorination of $\mathrm{C}_{10}-\mathrm{C}_{30}$ n-alkanes mixtures with molecular chlorine under forcing conditions [112-113]. Commercial CPs are classified into three categories: short chain $\left(\mathrm{C}_{10}-\mathrm{C}_{13}\right)$, medium chain $\left(\mathrm{C}_{14}-\mathrm{C}_{17}\right)$, and long chain $\left(\geq \mathrm{C}_{18}\right)$, and further sub-categorized based on their percent weight content of chlorine: 40-50 \%, 50-60\%, and 60-70 $\%$ [164]. Environmentally significant physical-chemical properties such as the vapor pressure (similar to other chlorinated organics like PCBs) and octanol-water partition coefficient $\left(\log \mathrm{K}_{\mathrm{ow}}\right.$ from 4.4 to >10) make these compounds persistent and bioaccumulative [112,114-115].

CP formulations are used for a wide range of applications. A 2003 survey of the uses and releases of CPs in Canada revealed that nearly all usage of short chain chlorinated paraffins (SCCPs) was in metalworking applications, with minor uses as flame retardants in plastics and rubber. Medium chain chlorinated paraffins (MCCPs) were mostly used in plastics and as lubrication additives, while long chain chlorinated paraffins (LCCPs) were mostly used as

\footnotetext{
iii This chapter is based on the paper by A.M. Muscalu, D. Morse, E.J. Reiner and T. Górecki published as "The quantification of short-chain chlorinated paraffins in sediment samples using comprehensive two-dimensional gas chromatography with $\mu$ ECD detection”, Anal. Bioanal. Chem. 409 (2016) 2065-2074.
} 
lubrication additives, as well as components of metalworking fluids and paints. Discharges into the environment can occur during production, transportation, use, storage and disposal of CPs. The releases are collected in sewer systems and often end up in the effluents of wastewater treatment plants. When released to the environment, CPs tend to partition primarily to sediment or soil [116-117]. Many studies detected and reported CPs in different environmental matrices: air [165], water [166-169], sediments and soil [170-173], biota [169,173-177], and waste [178]. The monitoring of SCCPs in environmental matrices is very important as they are chemicals proposed for listing under the Stockholm Convention [17], as well as classified as priority toxic substances under Canada's Environmental Protection Act [21]. Currently, Canada is in the process of implementing the prohibition of $\mathrm{C}_{10}-\mathrm{C}_{13}$ chlorinated alkanes, requiring reporting of any product manufactured in or imported into Canada that contains more that $0.5 \% \mathrm{CPs}$, while the Stockholm Convention proposer safer alternatives to SCCP use [179]. Risk management rules are also being developed for $\mathrm{C}_{14}-\mathrm{C}_{20}$ chlorinated alkanes [180-181].

The analysis of CPs is very difficult due to the very complex composition of the technical mixtures. Multi-step analytical methods including sample preparation (extraction and clean-up), instrumental analysis and extensive quantification procedures are usually required to analyze these compounds. Recent reviews have summarized the sample preparation procedures, as well as the instrumental and quantification techniques used in different laboratories. For the analysis of sediment samples, pressurized liquid extraction (PLE) and Soxhlet extraction were the preferred extraction techniques, Florisil, silica and alumina were the adsorbents used for extract clean-up, and GC-ECD and GC-MS (NCI-LRMS, NCI-HRMS and MS/MS) the instrumental techniques employed for analysis [119-120]. Due to the extremely large number of different isomers and homologues, the chromatographic separation of $\mathrm{CP}$ mixtures into individual 
congeners is not achievable; consequently, different quantification methods were developed to minimize any bias in quantifying CPs $[117,119-120,166,175]$.

Comprehensive two-dimensional gas chromatography is a separation method offering increased peak capacity and selectivity relative to conventional one dimensional separations [1]. $\mathrm{GC} \times \mathrm{GC}$ is gaining in popularity and the number of publications citing the use of this technique has increased significantly in recent years. $\mathrm{GC} \times \mathrm{GC}$ has proven to be a very powerful tool in the analysis of PCBs, OCs and CBs for solid samples such as soil and sediment. A GC×GC method developed in our laboratory reduced the number of sample preparation steps, improved data quality and eliminated fractionation of the extracts, all required with one dimensional analysis [27-28]. Korytar et al. [48,182] and later Xia et al [93] reported the great potential of using $\mathrm{GC} \times \mathrm{GC}$ for the analysis of $\mathrm{CPs}$. GC $\times \mathrm{GC}$ allowed easier group-type identification when multiple halogenated contaminants were tested. The improved separation of CP mixtures allowed partial characterization via resolution according to the number of chlorine substituents, group and subgroup separation, and ordered structures. Nevertheless, the available information is still limited as complete separation of $\mathrm{CP}$ standards is not possible. To take advantage of the separation power of $\mathrm{GC} \times \mathrm{GC}$, one of the objectives of this study was to develop a quantification method using GC $\times$ GC- $\mu$ ECD. In addition, the separation of SCCPs together with other targeted PCBs/OCs/CBs (118 target compounds) was assessed in order to quantify them all in a single GC $\times \mathrm{GC}$ run. Interlaboratory comparison samples were used to validate the procedure [183-184]. 


\subsection{Experimental}

\subsubsection{Standards and samples}

Chlorinated paraffin mixtures with different chain lengths and chlorination levels were purchased from Dr. Ehrenstorfer (Augsburg, Germany) as follows: three SCCP standard mixtures $\left(\mathrm{C}_{10-13}, 51,55.5\right.$ and $63 \%$ chlorine, $100 \mu \mathrm{g} / \mathrm{mL}$, in cyclohexane), three MCCP standard mixtures $\left(\mathrm{C}_{14-17}, 42,52\right.$ and $57 \%$ chlorine, $100 \mu \mathrm{g} / \mathrm{mL}$, in cyclohexane) and two LCCP standard mixtures $\left(\mathrm{C}_{18-20}, 36\right.$ and $49 \%$ chlorine, $100 \mu \mathrm{g} / \mathrm{mL}$, in cyclohexane). In addition, chloroparaffin mixtures $\left(\mathrm{C}_{10}, \mathrm{C}_{11}, \mathrm{C}_{12}\right.$ and $\mathrm{C}_{13}$ of different chlorination levels) were purchased from the same source. Chloroparaffin single analyte standards were acquired from Chiron (Trondheim, Norway) at concentrations of 100 or $1000 \mu \mathrm{g} / \mathrm{mL}$ in iso-octane (see Appendix C, Tables C.1 and C.2 for a complete list of standards).

Five levels of calibration standard solutions were prepared in iso-octane for all three SCCP standard mixtures to produce a calibration series with concentrations ranging from 100 to 5000 ng/mL total CP. All calibration solutions contained 4,4'-dibromo-octafluoro-biphenyl (5000 $\mu \mathrm{g} / \mathrm{mL}$ in methanol, Hewlett Packard) at a concentration of $10 \mathrm{ng} / \mathrm{mL}$ as an injection monitoring standard. Decachlorobiphenyl and 1,3,5-tribromobenzene $(1000 \mu \mathrm{g} / \mathrm{mL}$ in toluene and 100 $\mu \mathrm{g} / \mathrm{mL}$ in hexane, respectively; Canadian Life Science, Peterborough, Ontario, Canada) were used as recovery surrogates. In addition, a spiking solution of SCCPs containing $55.5 \%$ chlorine was prepared in acetone with a final concentration of $10 \mu \mathrm{g} / \mathrm{mL}$.

A portion of air-dried soil/sediment sample was extracted using accelerated solvent extraction (ASE, Dionex ${ }^{\mathrm{TM}}$ ASETM 350, Thermo Scientific, Bannockburn IL, USA). The ASE conditions were as follows: one cycle extraction at $100{ }^{\circ} \mathrm{C}$, heat time $5 \mathrm{~min}$., purge time $90 \mathrm{sec}$, flush volume $60 \%$, extraction solvent $25 \%$ dichloromethane / $75 \%$ hexane (v/v). The resulting 
extract was subjected to a single stage silica cartridge cleanup procedure and copper treatment to remove the sulfur interferences prior to instrumental analysis. No fractionation of extracts was required resulting in significant time and analysis costs savings. Quasimeme Interlaboratory Study [183-184] samples (Phase I and Phase III) were used to validate the method. The Phase I samples were used to check the feasibility of the calibration and quantitation procedures when processing "unknown" SCCPs standards. Phase III of the study included both standard and sediment samples (a cleaned-up extract and a raw extract). They were used for validating the GC×GC-ECD quantitation procedure.

\subsubsection{GC×GC System}

The GC $\times$ GC $-\mu$ ECD system (LECO Corp., St Joseph, MI, USA) used for this study consisted of an Agilent 6890 gas chromatograph (Agilent Technologies, Santa Clara, CA, USA) equipped with a split/splitless injector and a $\mu \mathrm{ECD}$ detector. The following chromatographic column combination was used: $30 \mathrm{~m}, 0.25 \mathrm{~mm}$ i.d., $0.25 \mu \mathrm{m}$ film thickness DB-1 (100\% dimethylpolysiloxane) from J\&W Scientific (Folsom, CA, USA) as the first dimension column, $1.6 \mathrm{~m}, 0.18 \mathrm{~mm}$ i.d., $0.18 \mu \mathrm{m}$ film thickness Rtx-PCB from Restek Corp. (Bellefonte, PA, U.S.A.) as the second dimension column, and a $0.3 \mathrm{~m}, 0.1 \mathrm{~mm}$ i.d. IP-Deact column (Restek Corp.) as the transfer line to the detector. The temperature programming for the primary oven started at $80^{\circ} \mathrm{C}$ (hold for $2 \mathrm{~min}$ ) to $160^{\circ} \mathrm{C}$ at $10^{\circ} \mathrm{C} / \mathrm{min}$, then to $280^{\circ} \mathrm{C}$ at $4{ }^{\circ} \mathrm{C} / \mathrm{min}$ (hold for $7 \mathrm{~min}$ ). The secondary oven had a $35^{\circ} \mathrm{C}$ temperature offset to the first dimension oven. The modulation period was 4 sec. with a modulator temperature offset of $30{ }^{\circ} \mathrm{C}$ to the first dimension oven. One microliter samples were injected in splitless mode using the auto-injector. The inlet temperature was 250 ${ }^{\circ} \mathrm{C}$, and a $4 \mathrm{~mm}$ i.d. gooseneck liner was used (Restek Corp.). Helium gas (Praxair, Mississauga, 
ON, Canada) with a purity of $99.999 \%$ was used as carrier gas at $1.2 \mathrm{~mL} / \mathrm{min}$ flow rate. The micro-ECD system was operated at $300{ }^{\circ} \mathrm{C}$ using data acquisition rate of $50 \mathrm{~Hz}$, with $5 \%$ methane in argon as the detector make-up gas at a flow-rate of $150 \mathrm{~mL} / \mathrm{min}$ [27]. ChromaTOF software (LECO Corp.) was used for data acquisition and processing for all analyzes performed for this study.

\subsection{Results and Discussion}

\subsubsection{Short Chain Chlorinated Paraffins Separation}

The proposed short chain chlorinated paraffins method is based on a GC $\times \mathrm{GC}-\mu \mathrm{ECD}$ method routinely used for the analysis of polychlorinated biphenyls (PCBs), organochlorine pesticides (OCs) and chlorobenzenes (CBs) in soil/sediment/sludge samples in which chlorinated paraffins were frequently detected. The SCCP peaks were located in a sparsely populated area of the $\mathrm{PCB} / \mathrm{OC} / \mathrm{CB} \mathrm{GC} \times \mathrm{GC}$ chromatogram; therefore, the same column combination was selected for this study [27]. The Rtx-PCB column used in the second dimension is coated with a shape selective stationary phase that separates the compounds based on planarity, with the more planar compounds being more retained in the 2D plane. To elute all the SCCP bands, the hold time for the last GC temperature ramp was increased by two minutes as compared to the routine $\mathrm{PCB} / \mathrm{OC} / \mathrm{CB}$ method.

Technical SCCP mixtures containing $51.5 \%, 55.5 \%$ and $63 \%$ chlorine were analyzed with

this column set-up and their ${ }^{1} \mathrm{D}$ and ${ }^{2} \mathrm{D}$ overlaid chromatograms are shown in Figure 3.1. The reconstructed ${ }^{1} \mathrm{D}$ chromatograms showed that little separation would be achieved if only $1 \mathrm{D}$ GC was used (Figure 3.1A). As expected, GC×GC separated the SCCPs into distinct structured bands with increased resolution compared to that of 1D GC, chromatograms as represented in Figure 
3.1 (B to D) for different levels of chlorination. The complete homologue separation was not possible with the DB-1 - Rtx-PCB column combination due to the complexity of SCCP mixtures. To further characterize and confirm the composition of the suspected chloroparaffin GC $\times \mathrm{GC}$ bands [48,93,120,182,32-34], mixtures of $\mathrm{C}_{10}, \mathrm{C}_{11}, \mathrm{C}_{12}$ and $\mathrm{C}_{13}$ chloroparaffins at different chlorination levels, as well as single chloroparaffin components were analyzed. The analyses confirmed that further sub-ordering of the bands occurred, with each band containing a constant number of carbon-plus-chlorine atoms $(\mathrm{C}+\mathrm{Cl})\left(\right.$ e.g. $(\mathrm{C}+\mathrm{Cl})_{15}$ contained $\left.\mathrm{C}_{11} \mathrm{Cl}_{4}+\mathrm{C}_{12} \mathrm{Cl}_{3}+\mathrm{C}_{13} \mathrm{Cl}_{2}\right)$. The positions of the individual CP congeners were overlaid with the SCCP mixes chromatograms (see Appendix C, Figure C.1 for the representation of the overlaid retention times) and further confirmed the constant $(\mathrm{C}+\mathrm{Cl})$ value in the bands. In addition, it was observed that not all the individual isomers available on the market were part of the technical mixes. Even though full characterization of the bands was not possible with this instrumental set-up, it did not impact the quantitation procedures. 


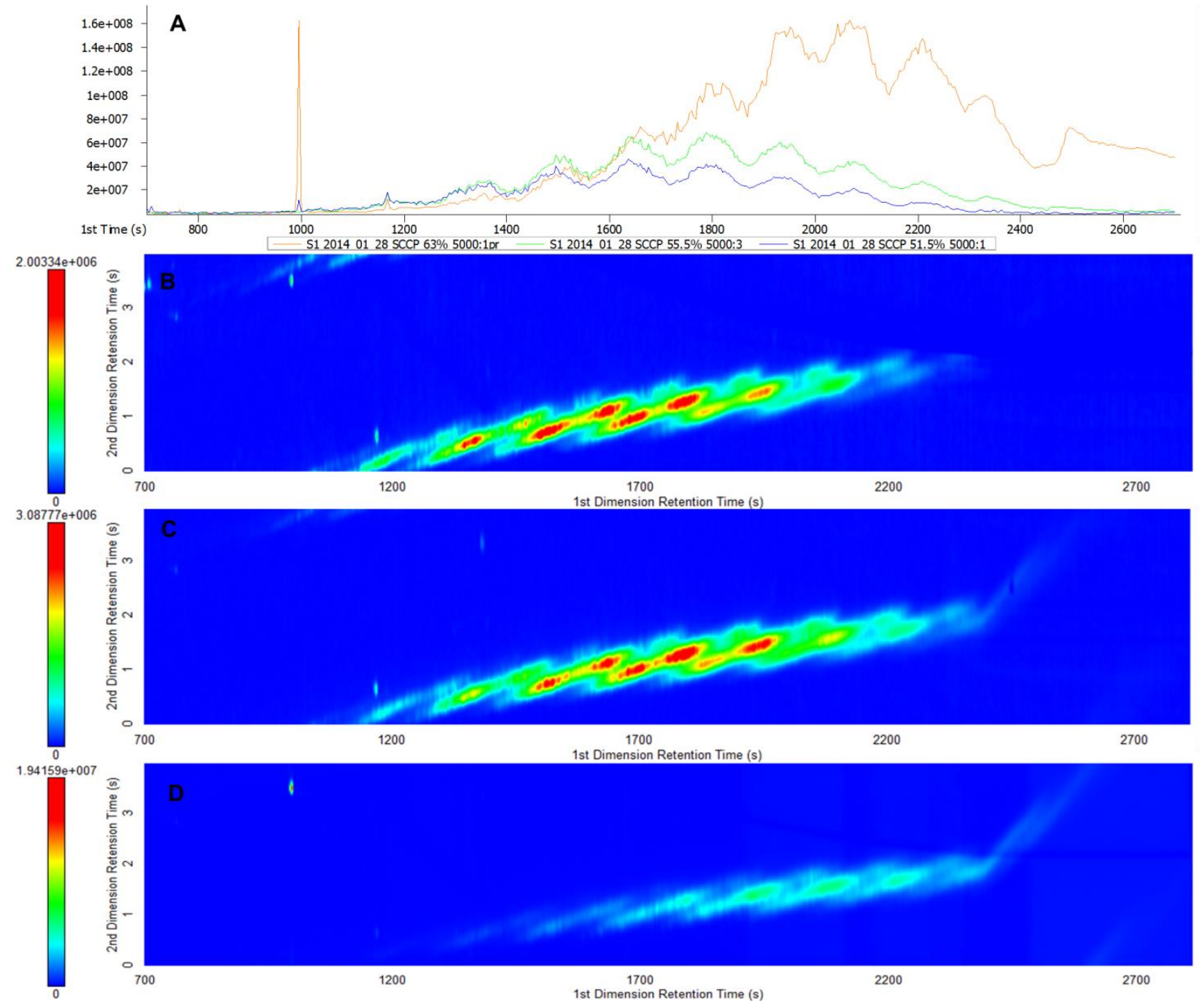

Figure 3.1 GC $\times$ GC- $\mu$ ECD chromatograms of SCCP by using DB1 x Rtx-PCB column set: A) Overlaid ${ }^{1} \mathrm{D}$ chromatograms of SCCPs $51.5 \%$ (blue line), $55.5 \%$ (green line) and $63 \% \mathrm{Cl}$ (orange line); B) $\mathrm{GC} \times \mathrm{GC}$ chromatogram of $\mathrm{SCCPs} 51.5 \% \mathrm{Cl}, \mathrm{C}$ ) $\mathrm{GC} \times \mathrm{GC}$ chromatogram of SCCPs $55.5 \% \mathrm{Cl}$, and C) GC×GC chromatogram of SCCPs $63 \% \mathrm{Cl}$.

\subsubsection{Quantitation Procedure}

Historically, PCBs have been quantified in environmental samples using an external standard approach with technical (Aroclor) formulations as standards. A number of the most intense peaks were selected for quantitation and the PCBs reported as PCB total. To achieve the optimal 
quantification with minimal error (bias), it is critical to match the PCB pattern in the sample with the technical standard mixture used for quantification by using the standard that best resembles the homologue pattern in the sample. The SCCP quantitation method developed in this research was based on a similar approach. Multilevel calibration curves were determined for each technical mix, and the SCCPs in the samples were quantified using the standard that represented the best match.

\subsubsection{Creating Classifications}

To take advantage of the structured nature of the SCCP chromatograms, the Classification feature of the ChromaTOF software was used to create classes of compounds based on their retention time characteristics. For data handling purposes, each class was named according to its corresponding $(\mathrm{C}+\mathrm{Cl})$ number. The classes ranged from $(\mathrm{C}+\mathrm{Cl})_{14}$ to $(\mathrm{C}+\mathrm{Cl})_{23}$, depending on the chlorine content of the technical mix. Figure 3.2 presents the classifications created for SCCP $55.5 \% \mathrm{Cl}$ mixture. The $(\mathrm{C}+\mathrm{Cl})_{22}$ band was wrapped-around (i.e. retention times of some components were longer than the modulation period), hence two separate classes were created denoted in Figure 3.2 as $(\mathrm{C}+\mathrm{Cl})_{22}-1$ and $(\mathrm{C}+\mathrm{Cl})_{22}-2$. The $(\mathrm{C}+\mathrm{Cl})_{23}$ class does not contain any peaks in Figure 3.2 as it was specific for the higher chlorinated standard, SCCP $63 \%$ chlorine. A new data processing method that included the classification was then created and used to reprocess the acquired samples. Once the samples were re-processed, the peaks were assigned to each corresponding class. The peak table could be filtered to display only the identified compounds within the classification regions to ease the process of peak review in each chromatogram. In addition to the peak table, a class summary table was generated by the software 
as a way to compare the amount of each class to the total mixture. The data produced through the summary displayed the total area count for each class (Table 3.1), as well as a total area count of the entire mixture. This information was used for setting up the quantitation procedure. As the ECD provided only retention time information and no mass spectral information, quantitation masses could not be selected for each class and calibration curves could not be generated through the ChromaTOF software. Thus, Excel was used as an alternative method to generate the multilevel calibration curves and perform the quantitation for each sample.

\begin{tabular}{|c|c|c|c|}
\hline \multirow{2}{*}{ Class } & \multicolumn{3}{|c|}{ Area } \\
\hline & SCCP $51.5 \%$ & SCCP $55.5 \%$ & SCCP $63 \%$ \\
\hline$(\mathrm{C}+\mathrm{Cl})_{14}$ & 0 & $1.76 \times 10^{6}$ & $7.71 \times 10^{3}$ \\
\hline$(\mathrm{C}+\mathrm{Cl})_{15}$ & $1.37 \times 10^{7}$ & $1.50 \times 10^{7}$ & $1.23 \times 10^{5}$ \\
\hline$(\mathrm{C}+\mathrm{Cl})_{16}$ & $3.61 \times 10^{7}$ & $4.91 \times 10^{7}$ & $5.89 \times 10^{5}$ \\
\hline$(\mathrm{C}+\mathrm{Cl})_{17}$ & $5.09 \times 10^{7}$ & $8.58 \times 10^{7}$ & $1.66 \times 10^{6}$ \\
\hline$(\mathrm{C}+\mathrm{Cl})_{18}$ & $5.58 \times 10^{7}$ & $1.06 \times 10^{8}$ & $3.90 \times 10^{6}$ \\
\hline$(\mathrm{C}+\mathrm{Cl})_{19}$ & $4.01 \times 10^{7}$ & $9.29 \times 10^{7}$ & $4.10 \times 10^{6}$ \\
\hline$(\mathrm{C}+\mathrm{Cl})_{20}$ & $2.19 \times 10^{7}$ & $6.41 \times 10^{7}$ & $3.16 \times 10^{6}$ \\
\hline$(\mathrm{C}+\mathrm{Cl})_{21}$ & $5.31 \times 10^{6}$ & $3.76 \times 10^{7}$ & $2.20 \times 10^{6}$ \\
\hline$(\mathrm{C}+\mathrm{Cl})_{22}$ & 0 & $5.23 \times 10^{6}$ & $6.42 \times 10^{5}$ \\
\hline$(\mathrm{C}+\mathrm{Cl})_{23}$ & 0 & 0 & $1.05 \times 10^{5}$ \\
\hline Total & $2.24 \times 10^{8}$ & $4.57 \times 10^{8}$ & $1.65 \times 10^{7}$ \\
\hline
\end{tabular}




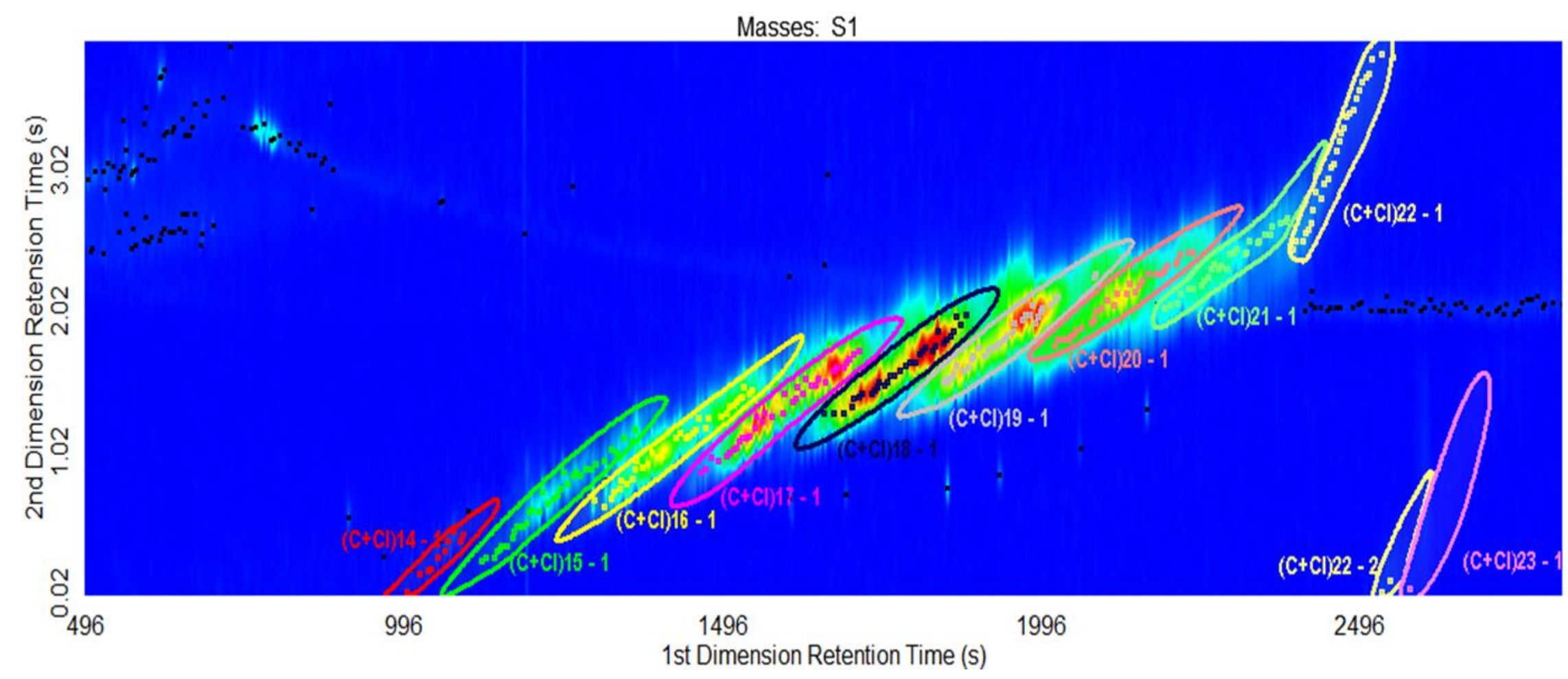

Figure 3.2 The classifications created for SCCP 55.5\% Cl mixture using the ChromaTOF software. 


\subsubsection{Calibration Procedure and Instrument/Method Performance}

Quantifying SCCPs is a challenging task as they are very complex mixtures that cannot be separated into individual components. To determine which standards were the most suitable for establishing the calibration curves, all the technical mixes, as well as the individual component standards were assessed. The first approach was to use the single chain $\mathrm{C}_{10}, \mathrm{C}_{11}, \mathrm{C}_{12}$ and $\mathrm{C}_{13}$ mixes with different chlorination levels as quantitation standards; however, since they could not be chromatographically resolved based on carbon chain lengths and/or chlorination level, they were unsuitable as quantitation standards. Also, additional peaks were observed in their chromatograms as compared to those of SCCPs technical mixes. The individual component standards that did not overlap the retention times of the technical mixes were assessed as possible internal standards. The response factors of isomers with the same carbon chain lengths and chlorine numbers were very different depending on the position of chlorine substitution (Table 3.2). Consequently, internal standard quantitation was not employed in order not to introduce bias in the calculations. The technical formulations of $\mathrm{C}_{10-13}$ with $51.5 \%, 55.5 \%$ and $63 \%$ chlorine were selected as the standards of choice instead. To build the five-level calibration curves used for quantification, SCCP standards ranging from 100 to $5000 \mathrm{ng} / \mathrm{mL}$ were analyzed in triplicate. The data files were processed using the data processing method that included the classifications. Each chromatogram was then thoroughly reviewed to make sure that all the peaks were included and properly integrated. Through the classifications summary view, the total area count of each band, as well as the total area of all the bands were generated for each replicate; this table was exported to Excel where linear calibration curves were created (see Appendix C, Table C.3). A linear calibration function was then applied to quantify all of the standards (see Table 3.3 for SCCPs $55.5 \% \mathrm{Cl}$ 
data). When quantifying the $100 \mathrm{ng} / \mathrm{mL}$ standards, it was observed that the calculated concentrations were around $36 \%$ of the target value. This bias could be explained after reviewing the low level standard chromatograms where it was noticed that some of the bands or peaks in the bands were not detected. Consequently, another calibration standard was introduced (5000 ng/mL) and the multi-level calibration range was modified to $200-5000 \mathrm{ng} / \mathrm{mL}$. To monitor the run-torun reproducibility as well as the contribution from matrix effects (if any), 4,4'-dibromooctafluoro-biphenyl at a concentration of $10 \mathrm{ng} / \mathrm{mL}$ was used as an injection monitoring standard.

\begin{tabular}{|l|c|c|c|}
\hline \multicolumn{1}{|c|}{ Table 3.2 Response factors for different CP isomers } \\
\hline \multicolumn{1}{|c|}{ Name } & ${ }^{1}$ D RT (s) & ${ }^{2}$ D RT (s) & Response Factors \\
\hline 1,1,1,3-Tetrachlorododecane & 1224 & 0.42 & $1.305 \times 10^{5}$ \\
1,2,11,12-Tetrachlorododecane & 1528 & 1.54 & $4.708 \times 10^{4}$ \\
\hline 1,1,1,3-Tetrachlorodecane & 964 & 3.98 & $1.297 \times 10^{5}$ \\
1,2,9,10-Tetrachlorodecane & 1252 & 1.24 & $4.563 \times 10^{4}$ \\
\hline 1,1,1,3-Tetrachloroundecane & 1092 & 0.22 & $1.087 \times 10^{5}$ \\
1,2,10,11-Tetrachloroundecane & 1388 & 1.42 & $5.157 \times 10^{4}$ \\
\hline
\end{tabular}

Following the set-up of the quantitation procedure and calibration functions, the method performance was tested by analyzing six fortified sediment samples (spikes). The sediment used for the spiked samples was previously analyzed and proven to be SCCP free. Three of the spike replicates were prepared by adding $0.5 \mathrm{~mL}$ of the spiking solution (SCCP $55.5 \% \mathrm{Cl}$ in acetone at $10 \mu \mathrm{g} / \mathrm{mL}$ ) to $5 \mathrm{~g}$ sediment sample to reach a final concentration of $1 \mu \mathrm{g} / \mathrm{mL}$. The other three spike replicates were prepared by adding $0.1 \mathrm{~mL}$ of the spiking solution (SCCP $55.5 \% \mathrm{Cl}$ in acetone at $10 \mu \mathrm{g} / \mathrm{mL}$ ) to $5 \mathrm{~g}$ sediment sample to reach a final concentration of $0.2 \mu \mathrm{g} / \mathrm{mL}$. The average 
recoveries for spikes were $128 \%$ for the $1 \mu \mathrm{g} / \mathrm{mL}$ and $136 \%$ for the $0.2 \mu \mathrm{g} / \mathrm{mL}$, while the $\%$ RSD were $4 \%$ and $2.5 \%$, respectively (Table 3.3).

Table 3.3 SCCP $55.5 \%$ chlorine - multilevel calibration standards and spike recoveries

\begin{tabular}{|c|c|c|c|c|c|}
\hline $\begin{array}{c}\text { Expected } \\
\text { Concentration } \\
(\mathrm{ng} / \mathrm{mL})\end{array}$ & Total Area & $\begin{array}{l}\text { Calculated } \\
\text { Concentration } \\
(\mathrm{ng} / \mathrm{mL})\end{array}$ & $\begin{array}{l}\text { Average } \\
(\mathrm{ng} / \mathrm{mL})\end{array}$ & $\begin{array}{c}\% \\
\text { Recoveries }\end{array}$ & $\begin{array}{c}\text { \% Rel. Std. } \\
\text { Dev. }\end{array}$ \\
\hline \multicolumn{6}{|c|}{ Multilevel Calibration (ng/mL) } \\
\hline \multirow[t]{3}{*}{100} & $1.02 \times 10^{8}$ & 35.7 & \multirow{3}{*}{36} & \multirow{3}{*}{35.9} & \multirow{3}{*}{3.3} \\
\hline & $1.01 \times 10^{8}$ & 34.8 & & & \\
\hline & $1.05 \times 10^{8}$ & 37.1 & & & \\
\hline \multirow[t]{3}{*}{200} & $4.57 \times 10^{8}$ & 213.5 & \multirow{3}{*}{214} & \multirow{3}{*}{107.2} & \multirow{3}{*}{4.3} \\
\hline & $4.41 \times 10^{8}$ & 205.5 & & & \\
\hline & $4.79 \times 10^{8}$ & 224.1 & & & \\
\hline \multirow[t]{3}{*}{500} & $1.13 \times 10^{9}$ & 548.6 & \multirow{3}{*}{538} & \multirow{3}{*}{107.5} & \multirow{3}{*}{1.8} \\
\hline & $1.09 \times 10^{9}$ & 531.1 & & & \\
\hline & $1.09 \times 10^{9}$ & 532.7 & & & \\
\hline \multirow[t]{3}{*}{1000} & $2.08 \times 10^{9}$ & 1026.9 & \multirow{3}{*}{1040} & \multirow{3}{*}{104.0} & \multirow{3}{*}{1.4} \\
\hline & $2.14 \times 10^{9}$ & 1055.1 & & & \\
\hline & $2.10 \times 10^{9}$ & 1037.1 & & & \\
\hline \multirow[t]{3}{*}{2000} & $4.02 \times 10^{9}$ & 1996.9 & \multirow{3}{*}{19705} & \multirow{3}{*}{98.6} & \multirow{3}{*}{2.3} \\
\hline & $3.99 \times 10^{9}$ & 1981.4 & & & \\
\hline & $3.90 \times 10^{9}$ & 1939.4 & & & \\
\hline \multicolumn{6}{|c|}{ Spikes@ 1000 ng/mL } \\
\hline Spk1 & $1.15 \times 10^{7}$ & 1251.4 & \multirow{3}{*}{1281} & \multirow{3}{*}{128.1} & \multirow{3}{*}{4.1} \\
\hline Spk2 & $1.23 \times 10^{7}$ & 1341.3 & & & \\
\hline Spk1 & $1.15 \times 10^{7}$ & 1250.8 & & & \\
\hline \multicolumn{6}{|c|}{ Spikes@200 ng/mL } \\
\hline Spk4 & $2.36 \times 10^{6}$ & 268.8 & \multirow{3}{*}{273} & \multirow{3}{*}{136.8} & \multirow{3}{*}{2.2} \\
\hline Spk5 & $2.36 \times 10^{6}$ & 271.9 & & & \\
\hline Spk6 & $2.47 \times 10^{6}$ & 280.3 & & & \\
\hline
\end{tabular}

\subsubsection{Interlaboratory study and sediment sample analyzes}

In order to verify the feasibility of the developed quantification approach before proceeding with the analysis of real samples, Quasimeme inter-laboratory study samples (Phase I and Phase 
III) were tested. The first phase was designed to compare results between different laboratories by analyzing the total SCCP concentrations and the concentration of three individual CPs in three samples. As the method developed was intended for the total SCCPs only, the individual CPs could not be quantified [183]. The two analyzed samples were run in duplicate: one of the ampules (sample 1) best resembled the SCCP $51.5 \% \mathrm{Cl}$ mix and showed an average accuracy of $93 \%$, while the second ampule (sample 2) was identified as a SCCP $55.5 \%$ standard and showed an average accuracy of $89 \%$ (Figure 3.3). It is very important to find the best match to the standard as bias in the calculated results can be significant if the SCCP profile is misinterpreted. 


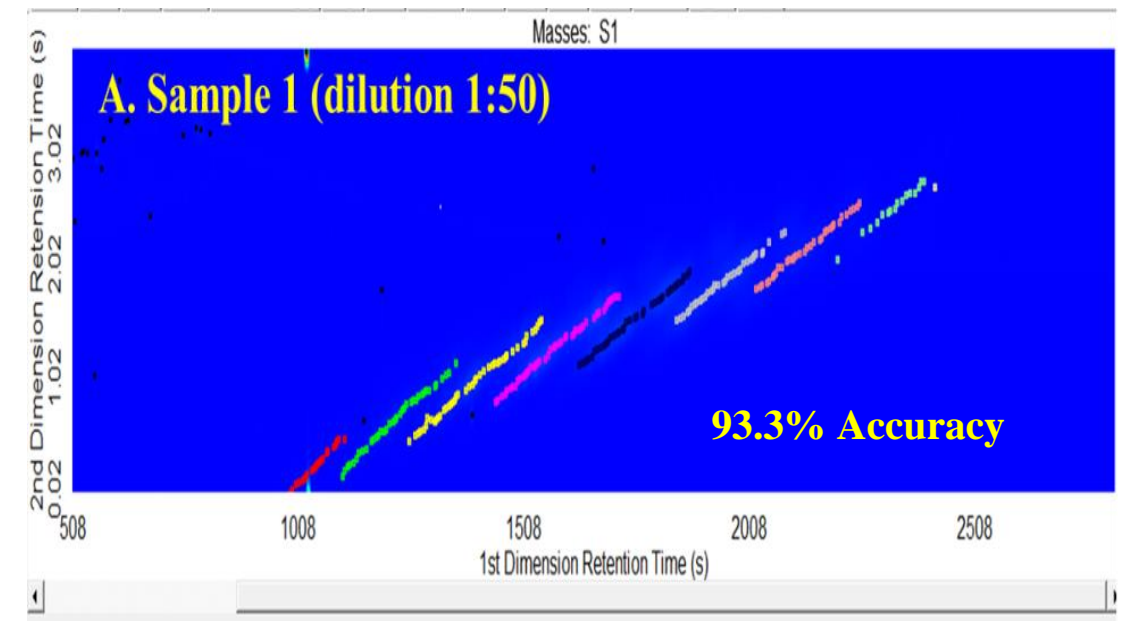

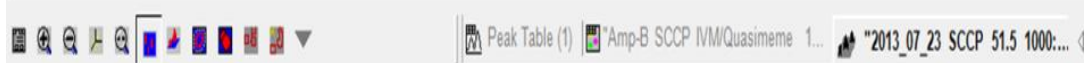

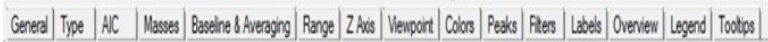

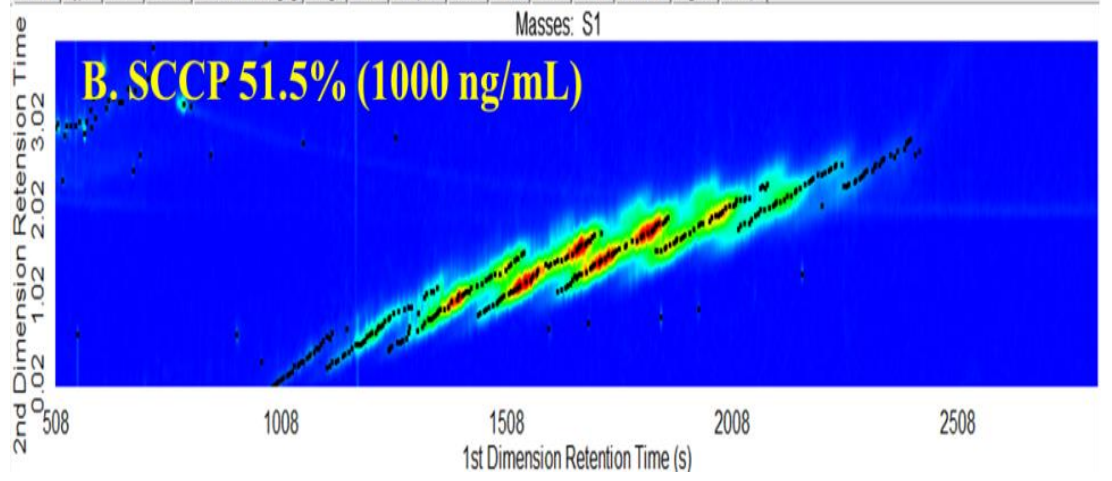

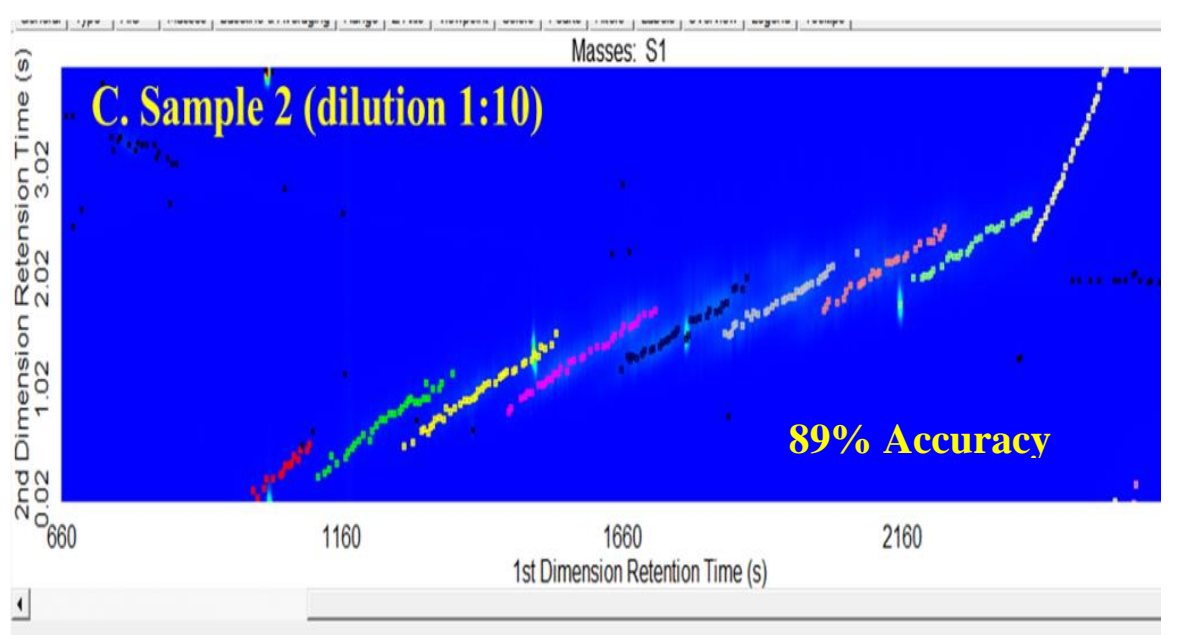

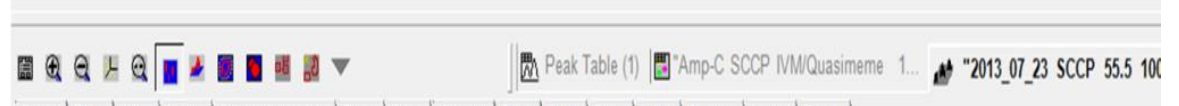

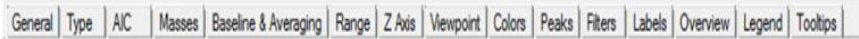

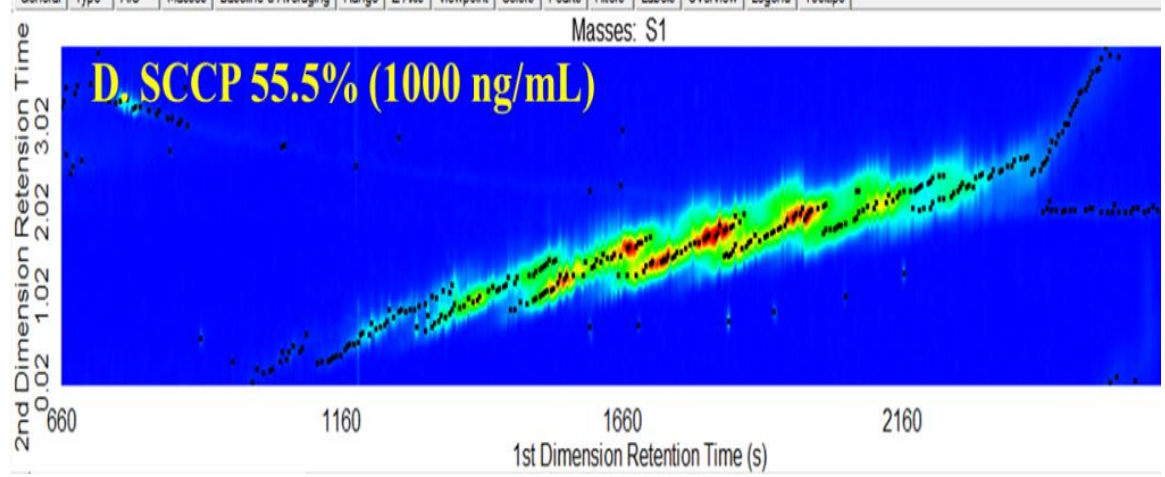

Figure 3.3 Quasiememe Phase I - GC×GC- $\mu$ ECD chromatograms: A) sample 1, B) SCCP $51.5 \%$ Cl standard mix at $1000 \mathrm{ng} / \mathrm{mL}, \mathrm{C}$ ) sample 2 and D) SCCP $55.5 \%$ standard mix at $1000 \mathrm{ng} / \mathrm{mL}$. 
The third phase of the Quasimeme study [184] was designed to compare results for SCCPs in sediment samples. The aim of participating in this study was to validate the method for testing real samples. Three ampules were received: one containing an SCCP standard to be used for quantification purposes (sample 1), a second ampule containing a cleaned sediment extract (sample 2), and a third ampule containing a raw sediment extract (sample 3). The raw sediment extract along with a blank sample were cleaned-up using $1 \mathrm{~g}$ silica cartridges followed by copper treatment to remove sulfur interferences [28], as presented in the experimental section. The SCCP standard solution (sample 1) was further diluted to five solutions that were used to build a multilevel calibration ranging from 240 to $4730 \mathrm{ng} / \mathrm{mL}$. The blank and the remaining samples, sample 2 and 3, were analyzed in triplicate and quantified by using both the in-house standards and sample 1. When quantified against the in-house standard, both samples 2 and 3 were identified as SCCP 63 $\% \mathrm{Cl}$; thus, the multilevel calibration curve and the linear calibration equation based on SCCP 63 $\% \mathrm{Cl}$ mix were used (Figure 3.4). The results showed that the method performed very well regardless of the standards used (in-house or ampule 1). The z-scores for both samples were less than 2 . When the sample 1 standard was used for quantification, the average recoveries were $76 \%$ for sample 1 and $77 \%$ for sample 2 . The results were similar when the in-house standard was used, $75 \%$ for sample 1 and $77 \%$ for sample 2.

Following validation, the quantification technique was applied to samples previously analyzed using the routine $\mathrm{OC} / \mathrm{PCB} / \mathrm{CB}$ GC $\times \mathrm{GC}$ method [27-28], in which SCPPs were detected in the chromatograms. An example of a $4 \mathrm{~g}$ extracted sediment ( $1 \mathrm{~mL}$ final volume) is presented in Appendix C, Examples C.1 and c.2. The pattern was identified as SCCP $55.5 \%$, and the concentration of the total SCCP in the sediment sample was $480 \mathrm{ng} / \mathrm{g}$. 

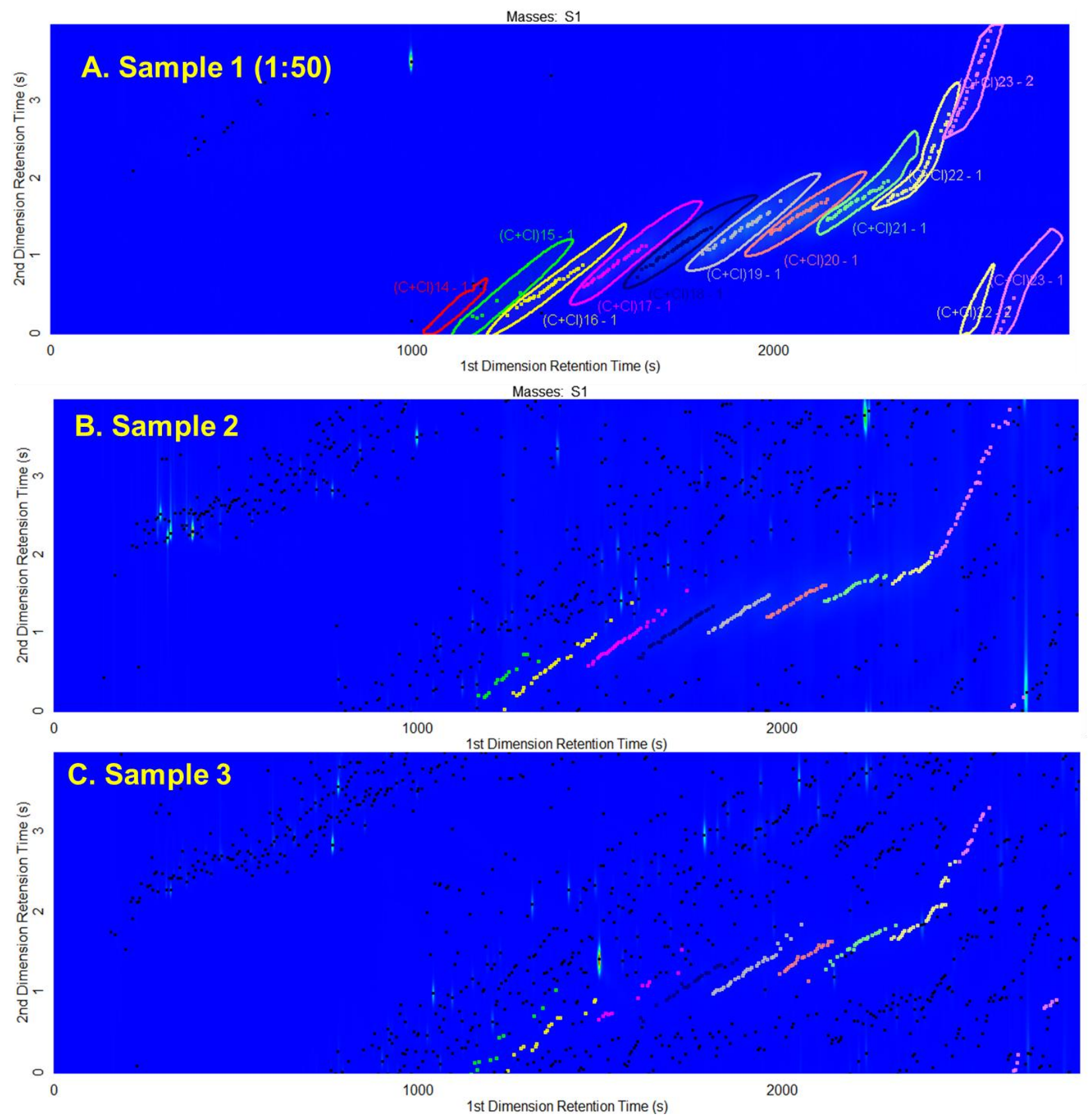

Figure 3.4 Quasiememe Phase III - GC×GC- $\mu$ ECD chromatograms: A) sample 1, B) Sample 2 and C) sample 3. 


\subsubsection{Analytical Challenges}

As one of the aims of this method was to quantify the SCCPs along with other contaminants, the separation of SCCPs from the other target analytes (PCB/OC/CB; 118 target compounds) was assessed. The preliminary analyses indicated that by using the DB-1 x Rtx-PCB column combination, good between-class separation was achieved with the exception of a few higher chlorinated PCBs (PCB206, PCB 207, PCB208 and PCB209) that interfered with SCCPs (Figure 3.5A). However, unless the SCCPs were present in the samples at very high levels, the PCBs could be distinguished in the bands as more intense peaks and subtracted from the total SCCP calculations. If the above mentioned PCBs were confirmed in the samples, the contribution of the SCCPs to the co-eluting PCB concentration was insignificant.

SCCP could not be properly differentiated from the other technical mixes when present at the same time in the sample. The overlapping retention times of short and medium chain CPs represented in Figure 3.5B showed their elution pattern when using this column combination. The MCCPs could be easily identified and their presence confirmed in the samples as they were less retained in the second dimension plane. Similarly, LCCPs retention times overlapped those of MCCP.

Another drawback of this method was that the data processing was very time consuming. Each chromatogram had to be thoroughly reviewed to make sure that each peak/slice was properly integrated. As a result, more than 250 slices were integrated for each standard. The calibration curves could not be set up directly through the ChromaTOF software; thus, more data handling was required as the files had to be exported to Excel. 


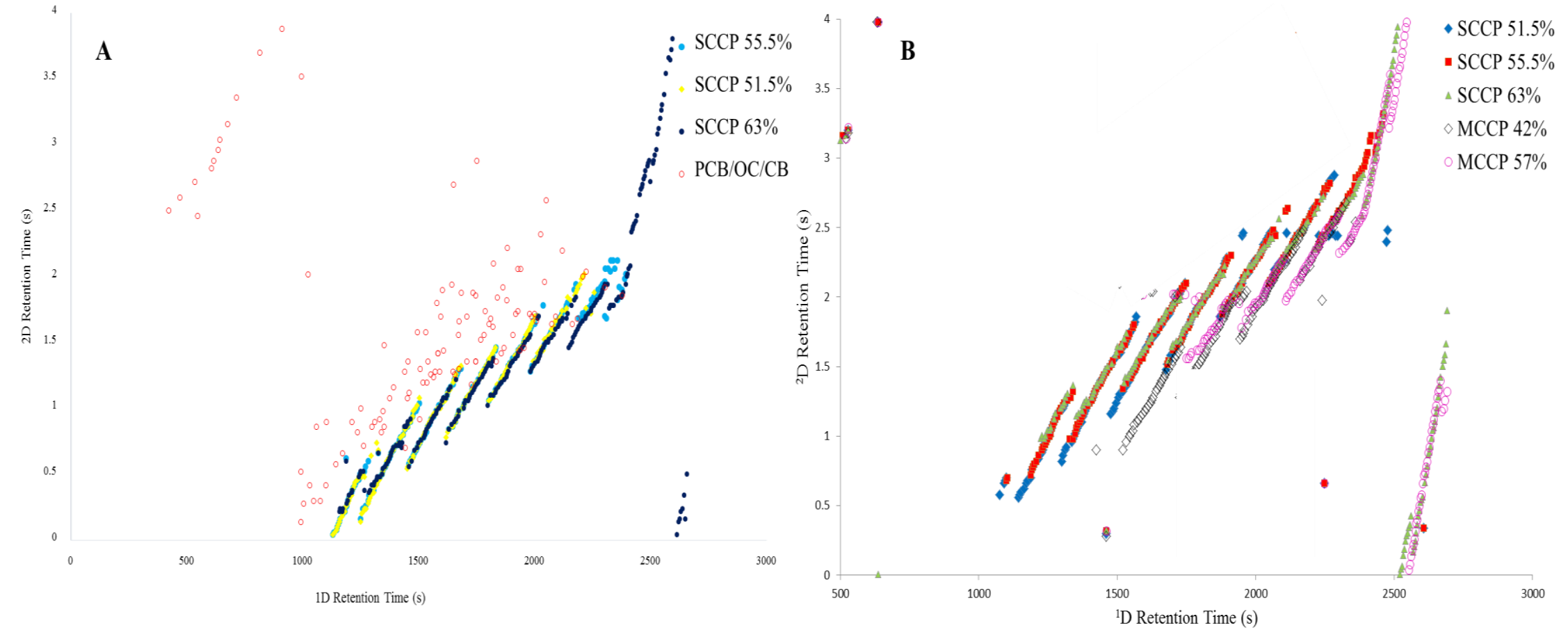

Figure 3.5 Overlaid retention times of A) $\mathrm{PCB} / \mathrm{OC} / \mathrm{CB}$ and SCCP at different chlorination levels, and B) SCCPS and MCCPs at different chlorination levels. 


\subsection{Conclusions}

The GC $\times$ GC- $\mu$ ECD method developed for the separation and quantitation of the SCCPs complex mixture was successfully applied to sediment samples. SCCPs were separated in distinct ordered bands when using a technique such as $\mathrm{GC} \times \mathrm{GC}$ by pointing to group separation. By using the ChromaTOF software and its Classification function combined with Excel to generate summary tables and calibration curves for different technical mixes, novel quantification procedures were developed. The method's performance was initially demonstrated on fortified sediment samples and further validated by participating in inter-laboratory studies for the analysis of standards and sediment samples. The results showed recoveries between $75 \%$ to $95 \%$ and zscore values $<2$, demonstrating that the method is suitable for the analysis of SCCPs in soil/sediment samples.

As the analysis of environmental samples is very complex, this method proved to be efficient when multiple classes of compounds are present at the same time. The GC $\times \mathrm{GC}$ chromatograms of samples analyzed routinely for other halogenated compounds such as PCBs [28] can now be sorted and data further processed when SCCPs are found. However, further research is still needed as the CP technical mixes are very complex. 


\section{Chapter 4}

\section{Non-targeted Analysis of Hydroxylated Polychlorinated Biphenyls in Storm Sewer Water Using Comprehensive Two-Dimensional Gas Chromatography with Micro-electron Capture Detection and Time-of-flight Mass Spectrometry $^{\text {iv }}$}

Polychlorinated biphenyls (PCBs) are persistent pollutants used historically for a variety of industrial and commercial purposes. They tend to bioaccumulate and biomagnify in the environment and have toxic effects on wildlife and humans. PCBs were manufactured on the industrial scale by chlorination of biphenyl. The resultant mixtures containing up to 209 possible congeners were sold in the USA by Monsanto under the trade name Aroclor. PCBs were primarily used as dielectric fluids in capacitors or transformers or added to other products such as paints, plastics, adhesives, paper and inks [185]. Even though production of PCBs was banned by most countries in the late 1970 s, they are still present in many historically-produced electrical devices or unintentionally produced by modern industry as by-products of different manufacturing processes, such as pigment production [186] or polymer sealant manufacturing [187]. PCBs do not easily degrade and as such have been widely distributed in the environment; however, under some conditions, they can be metabolized to other classes of persistent, toxic contaminants.

Mono-hydroxylated polychlorinated biphenyls (OH-PCBs), a group of 837 possible congeners, are PCB metabolites produced by both biological and abiotic mechanisms [121-122].

\footnotetext{
iv This chapter is based on the manuscript by A.M. Muscalu, D. Morse, E.J. Reiner and T. Górecki, to be submitted for publication as "Non-targeted Analysis of Hydroxylated Polychlorinated Biphenyls in Storm Sewer Water Using Comprehensive Two-Dimensional Gas Chromatography with Micro-electron Capture Detection and Time-of-flight Mass Spectrometry".
} 
Some of the formation pathways of OH-PCBs include the oxidation of PCBs via cytochrome P450 (CYP) in biota [121123-124,187-188], potential oxidation of PCBs in the gas phase by hydroxyl radicals $(\bullet \mathrm{OH})$ in natural environments [121,125], transformation in waste water treatment plants [121] or degradation by microorganisms [125,190], photochemical formation [208]. Marek et al. reported the presence of OH-PCBs in the original Aroclor formulations, emphasizing that $\mathrm{OH}-$ PCB contamination exists in sediments any place that PCB contamination from Aroclor is present [131]. A toxicokinetics study by Buckman et al. [191] also suggested that fish may accumulate a significant amount of OH-PCB metabolites from sources other than biotransformation from parent PCB congeners and/or are produced over a longer period of time than assessed in the study at low temperatures. Hydroxylated PCBs have been studied and reported in humans [127-129,192-196] and different environmental matrices such as biota [197-207], sediments [131], sludges [132], air [133-134], water and precipitation [125].

Toxicological effects of OH-PCBs were also studied for both humans and wildlife, multiple reports showing that some OH-PCBs are more toxic than their parent compounds [121-122,209212]. Using the bacterial bioluminescent assay Microtox ${ }^{\circledR}$, Bhalla et al. [211] also showed that the toxicity of OH-PCBs generally decreased with the increase of the chlorination level. Due to their structural similarity to the thyroid hormones (thyroxine and triiodothyronine), these compounds showed binding affinity with the transport protein transthyretin (TTR) resulting in the disruption of the transport and metabolism of thyroid hormones and showing neurodevelopmental and behavioral effects [121-122,194,213-217]. Hydroxylated PCBs were capable of other multiple adverse effects such as gap junction inhibition which may play a role in tumor promotion, AhRmediated activity, and (anti)estrogenicity [209,218-221]. In addition, in female polar bears, $\mathrm{OH}-$ 
PCBs may affect the circulating levels of steroids, thus may interfere with the steroid homeostasis [222].

The analysis of OH-PCBs is very complex due to their physico-chemical properties, sample preparation required, and instrumental analysis methods. In addition, the availability of commercial standards is limited with only about 80 standards out of 837 possible congeners being available. The chromatographic separation and quantitation have been described in many studies. For instance, Ueno et al. developed an isotope dilution gas chromatography coupled with high resolution mass spectrometry (GC-HRMS) method to provide full characterization of OH-PCBs [125]. Marek et al. used GC-tandem mass spectrometry (GC-MS/MS) for congener-specific analysis [131], while Eguchi et al. developed a comprehensive and highly sensitive analysis method for complex biological tissues (liver and brain) by using GC/ECNI-MS and/or GC/EIHRMS [136]. Kania-Korwel et al. reported the enantioselective separation of OH-PCBs by using GC with different chiral cyclodextrin phases and demonstrating for the first time that chiral PCBs are enantioselectively metabolized to OH-PCBs by cytochrome P450 enzymes [188].

This study was focused on the semi-quantitative analysis of hydroxylated PCBs in storm sewer water samples by using comprehensive two-dimensional gas chromatography $(\mathrm{GC} \times \mathrm{GC})$. For this purpose, we wanted to confirm that the unknown peaks observed in specific areas of the two-dimensional chromatograms were hydroxylated PCBs, and also conduct an initial assessment of their possible concentration in the samples. When assessing their levels, we had to consider that the extraction and clean-up methods were not optimized for these types of compounds and no sample was available for re-extraction. In addition, we wanted to emphasize the importance of using a technique such as $\mathrm{GC} \times \mathrm{GC}-\mu \mathrm{ECD}$ for routine analysis to further identify non-targeted halogenated contaminants present in environmental samples. The enhanced selectivity of GC×GC 
enables the less selective micro electron capture detector ( $\mu \mathrm{ECD}$ ) to be used for routine target compound analysis and also for assessing the presence of non-target contaminants. This routine, targeted PCB analysis method has capabilities of non-target analysis; if $\mathrm{GC} \times \mathrm{GC}$ was not employed for water samples analysis, we would not have suspected the presence of OH-PCBs and other contaminants such as polychlorinated naphthalenes (PCNs) at the collection site.

\subsection{Materials and Methods}

\subsubsection{Standards}

For this initial study, a total of ten hydroxylated PCB standards were purchased from AccuStandard (CT, USA) and Wellington Laboratories Inc. (ON, Canada). To obtain mass spectral confirmation and assess the chromatographic separation, the standards were selected based on different chlorination levels ranging from di- to hepta-chlorinated OH-PCBs: 4-hydroxy-4'-

chlorobiphenyl, 2-hydroxy-2',3'-dichlorobiphenyl, 4-hydroxy-2,2',5'-trichlorobiphenyl, 4hydroxy-2',3',4',5'-tetrachlorobiphenyl, 3-hydroxy-2,3',4',5,5'-pentachlorobiphenyl, 3-hydroxy2,3',4,4',5-pentachlorobiphenyl, 4-hydroxy-2,2',3,4',5,5'-hexachlorobiphenyl, 4'-hydroxy2,2',3,3',4,5,5'-heptachlorobiphenyl, 3'-hydroxy-2,2',3,4,4',5,5'-heptachlorobiphenyl, 4-hydroxy2,2',3,4',5,5',6-heptachlorobiphenyl. Also, when selecting the standards, the molecule's planarity was considered in order to assess the chromatographic separation and instrument response, as well as the frequency of detection as reported in different published papers. Methylated versions of each individual OH-PCB standard were prepared in-house by derivatization reaction with diazomethane. Mixed solutions of OH-PCB standards were prepared both in iso-octane and acetone and were used for multi-level calibration of the instruments and as a spiking solution, respectively. The multilevel level calibration standard solutions ranged from 10 to $500 \mathrm{ng} / \mathrm{mL}$ for 
GC $\times$ GC- $\mu \mathrm{ECD}$ and from 10 to $5000 \mathrm{ng} / \mathrm{mL}$ for GC $\times \mathrm{GC}-\mathrm{TOFMS}$, while the spiking solution was diluted to a final concentration of 1 to $5 \mu \mathrm{g} / \mathrm{mL}$. Polychlorinated diphenyl ether (PCDE) standards purchased from Wellington Laboratories were analyzed to obtain retention time and mass spectral information.

\subsubsection{Sample Collection and Preparation}

Water samples were collected on February 26, 2013, from three storm sewer catch basins in an urbanized area of southwestern Ontario. The sewers were a maximum distance of $165 \mathrm{~m}$ from one another. Water samples were collected directly into $2 \times 1 \mathrm{~L}$ amber glass bottles. The samples were extracted using an automated solid-phase extraction unit (SPE-DEX® 4790, Horizon Technology Inc., NH, USA) and $47 \mathrm{~mm}$ Atlantic $^{\mathrm{TM}}$ HLB-M SPE disks (Horizon Technology Inc., NH, USA). A volume of $800 \mathrm{~mL}$ of a water sample was passed through the preconditioned disks (methanol, acetone and water), then extracted with $25 \%$ dichloromethane in hexane. The resulting extract was dried using sodium sulphate and then subjected to a single stage silica cartridge cleanup procedure $(1 \mathrm{~g}$, Agilent, ON, Canada; eluted with $15 \mathrm{~mL}$ of $25 \%$ dichloromethane in hexane) prior to instrumental analysis. This method could include copper treatment to remove extraneous background interferences. The extracts were then evaporated to 1 $\mathrm{mL}$ final volume in iso-octane using a Zymark Turbovap LV evaporating system (Zymark Corp., MA, USA).

As hydroxylated-PCBs were not part of the target compound list for the routine analyses, the extraction and clean-up procedures were not optimized for them at the time of analysis. After detecting the $\mathrm{OH}-\mathrm{PCBs}$, recovery tests were performed to assess the method's efficiency for these compounds. The extraction and clean-up procedures were evaluated independently. Water blanks 
(3 replicates, $800 \mathrm{~mL}$, deionized water) were spiked with $200 \mu \mathrm{L}$ of the $\mathrm{OH}-\mathrm{PCB}$ spiking solution to a final concentration of 200 to $1000 \mathrm{ng} / \mathrm{mL}$. The spiked samples were passed through the preconditioned SPE extraction disks and eluted with $10 \mathrm{~mL}$ acetone and $15 \mathrm{~mL}$ of $25 \%$ dichloromethane in hexane. Sodium sulphate treatment was applied to all the samples and the final extracts were concentrated to $1 \mathrm{~mL}$ final volume (iso-octane final solvent). Similarly, $1 \mathrm{~mL}$ of isooctane was spiked with the same amount of spiking solution, passed through the pre-conditioned clean-up cartridges and eluted with $15 \mathrm{~mL}$ of $25 \%$ dichloromethane in hexane. The extracts were then evaporated to $1 \mathrm{~mL}$ final volume in iso-octane. All the recovery tests extracts were analyzed by $\mathrm{GC} \times \mathrm{GC}-\mu \mathrm{ECD}$, while the 'real-life' samples were analyzed by both $\mathrm{GC} \times \mathrm{GC}-\mu \mathrm{ECD}$ and GC $\times$ GC-TOFMS. For further confirmation, derivatization using diazomethane was performed for all the standards and water samples analyzed by GC $\times$ GC-TOFMS. The data presented in this paper discusses the findings for three water samples collected from neighbouring sewers; however, the findings are similar for other samples that we have analyzed from other locations that presented positive identification of OH-PCBs. A further study employing appropriate sample preparation and quantitation procedures will follow our initial findings.

\subsubsection{Instrumental Analysis}

The two GC $\times$ GC systems used for this study (LECO Corp., St Joseph, MI, USA) consisted of an Agilent 6890 gas chromatograph (Agilent Technologies, Santa Clara, CA, USA) equipped with a split/splitless injector, a cryogenic free modulator (LECO), and a $\mu$ ECD or a TOFMS detector (Pegasus IV, LECO Corp., St Joseph, MI, USA), respectively. The following chromatographic column combination was used: $30 \mathrm{~m}, 0.25 \mathrm{~mm}$ i.d., $0.25 \mu \mathrm{m}$ film thickness DB1 (100 \% dimethylpolysiloxane) from J\&W Scientific (Folsom, CA, USA) as the first dimension 
column, followed by $1.6 \mathrm{~m}, 0.18 \mathrm{~mm}$ i.d., $0.18 \mu \mathrm{m}$ film thickness Rtx-PCB from Restek Corp. (Bellefonte, PA, U.S.A.) as the second dimension column. For the $\mu$ ECD system, a $0.3 \mathrm{~m}, 0.1 \mathrm{~mm}$ i.d. IP-Deact (Restek Corp.) column was used as the transfer line to the detector. The temperature programming for the primary oven started at $80{ }^{\circ} \mathrm{C}$ (held for $2 \mathrm{~min}$ ) to $160^{\circ} \mathrm{C}$ at $10^{\circ} \mathrm{C} / \mathrm{min}$, then to $280{ }^{\circ} \mathrm{C}$ at $4{ }^{\circ} \mathrm{C} / \mathrm{min}$ (held for $7 \mathrm{~min}$ ). The secondary oven had a $30^{\circ} \mathrm{C}$ temperature offset to the first dimension oven. The modulation period was 4 sec. (hot jet and cold jet) with a modulator temperature offset of $35^{\circ} \mathrm{C}$ to the first dimension oven. The injection volumes (splitless mode) of the final extract were $1 \mu \mathrm{L}$ for $\mathrm{GC} \times \mathrm{GC}-\mu \mathrm{ECD}$ and $2 \mu \mathrm{L}$ for $\mathrm{GC} \times \mathrm{GC}$-TOFMS, before and after sample derivatization. The inlet temperature was $250^{\circ} \mathrm{C}$, and a $4 \mathrm{~mm}$ i.d. gooseneck liner was used (Restek Corp.). Helium gas (Praxair, Mississauga, ON, Canada) with a purity of $99.999 \%$ was used as carrier gas at $1.2 \mathrm{~mL} / \mathrm{min}$ flow rate. The micro-ECD system was operated at $300{ }^{\circ} \mathrm{C}$ using data acquisition rate of $50 \mathrm{~Hz}$, with $5 \%$ methane in argon as the detector make-up gas at a flow rate of $150 \mathrm{~mL} / \mathrm{min}$. ChromaTOF software (LECO Corp.) was used for data acquisition and processing for all analyzes performed for this study. The details of the chromatographic separations of the targeted compounds (PCBs, organochlorine pesticides and chlorobenzenes) were reported in a previous paper [28].

\subsection{Results and Discussion}

\subsubsection{Discovery of OH-PCB in sewer water samples}

The sewer water samples were analyzed for PCBs by using a GC $\times$ GC- $\mu$ ECD congenerspecific method that targeted eighty PCBs [223]. For the water samples discussed in this study, the sum of all identified PCB congeners (PCB total) was reported as $6300 \mathrm{ng} / \mathrm{L}$ for sample \#1, $7600 \mathrm{ng} / \mathrm{L}$ for sample \#2 and $900 \mathrm{ng} / \mathrm{L}$ for sample \#3. 
The PCB congener profiles of the water samples were compared to Aroclor profiles as reported by Frame et al. [224]. The samples had PCB congener profiles similar to a mixed Aroclor profile consisting of a high proportion of Aroclor 1242 followed by Aroclor 1254, and for samples $\# 1$ and \#3, a minor contribution of Aroclor 1260 (Figure 4. 1). The PCB congener profile observed for sample \#3 (Figure 4. 1C) was a very close match to an unaltered PCB technical mixture of 50 $\%$ A1242 + $45 \%$ A1254 + $5 \%$ A1260; however, the congener profiles for sample \#1 (Figure 4. 1A) and sample \#2 (Figure 4. 1B) demonstrated greater divergence from a technical Aroclor profile mixture relative to sample \#3. For samples \#1 and \#2, several congeners were reported in proportions both above and below those expected if the congener profiles in the samples reflected an Aroclor profile consisting of A1242, A1254g and A1260. When compared to a technical Aroclor mix consisting of $55 \%$ Aroclor 1242, $45 \%$ Aroclor 1254g and $5 \%$ Aroclor 1260 for sample \#1 (Figure 4. 1A) and $80 \%$ Aroclor 1242 and $20 \%$ Aroclor $1254 \mathrm{~g}$ for sample \#2 (Figure 4. 1B), their closest match, the PCB congener profile for these two samples showed that several PCB congeners were reported in proportions above those expected. The PCBs in apparent anomalous proportions included PCB16, PCB19, PCB28/PCB31 (co-eluting PCBs), PCB49, PCB70, PCB74, PCB84, PCB90/PCB101 (co-eluting PCBs) and PCB99. Reductive dechlorination was initially considered as a potential explanation for the divergence of the sample profiles from the mixed Aroclor profiles; however, many of the previously noted PCB congeners are not known PCB dechlorination daughter products [225], and for those that are dechlorination products, a proportional loss of a parent PCB congener could not be determined. 

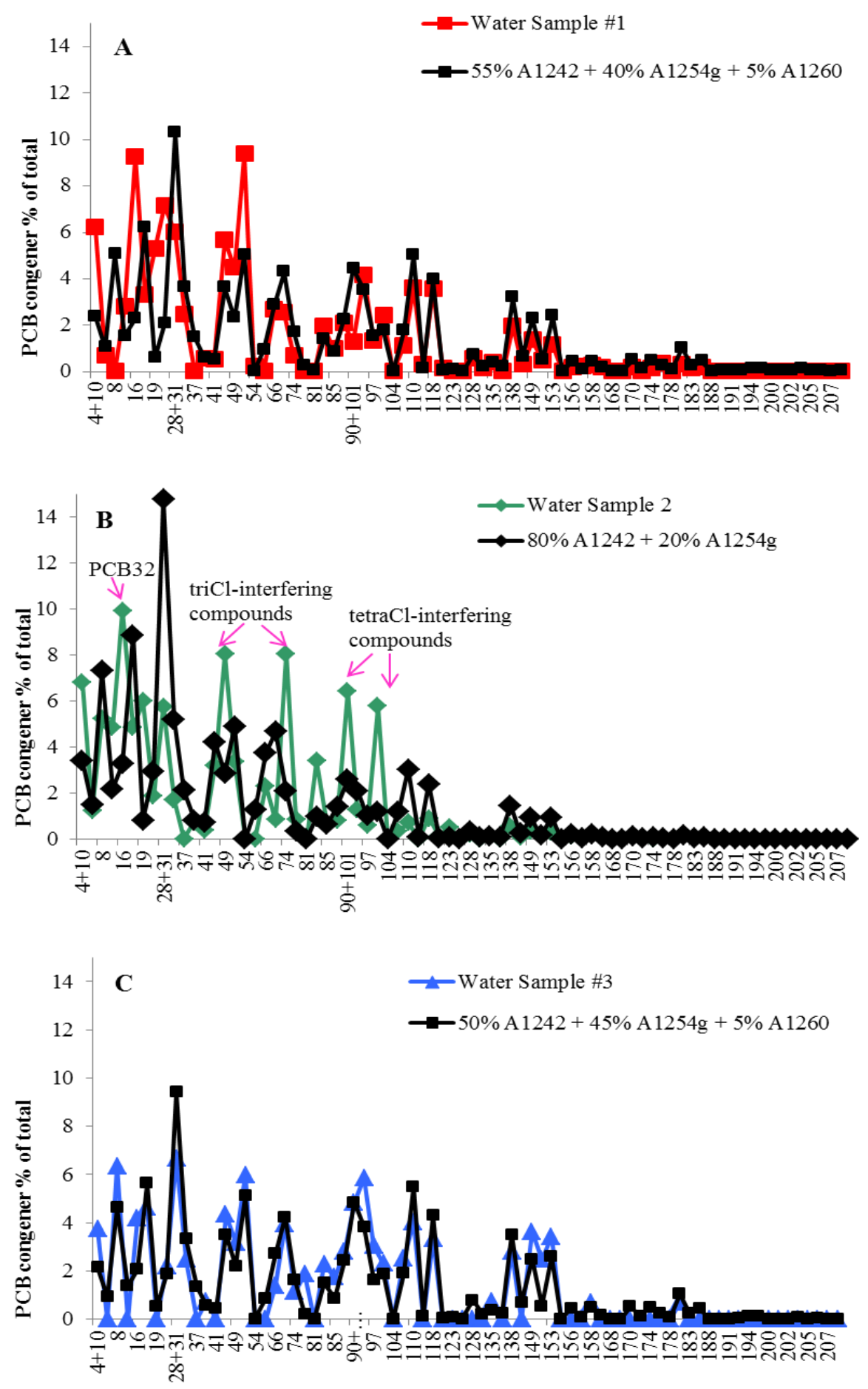

Figure 4. 1 Aroclor profile for the three water samples: A. Sample 1, B. Sample 2, C. Sample 3 (Aroclor plots courtesy of Tanya Long, MECP) 
A closer look at the $\mathrm{GC} \times \mathrm{GC}-\mu \mathrm{ECD}$ chromatogram revealed the presence of other classes of halogenated contaminants that eluted very close to PCBs' retention times (Figure 4.2A). MicroECD, a very sensitive but not specific detector, could not provide further information for characterizing the unknown compounds. To verify the reported results for PCBs and potentially identify the interfering compounds, the water samples were further analyzed by GC×GC-TOFMS. The GC $\times$ GC-TOFMS analyses revealed that the sample contained high concentrations of polychlorinated naphthalenes (PCNs) as shown in Figure 4.2B, but anomalous proportions of only two PCBs: PCB 8 and PCB 19, could be explained by the interference from tri-chlorinated naphthalenes. Most of the remaining interfering peaks did not return any matches when compared to the NIST libraries, but the chromatographic separation patterns were very similar to those of PCBs. The mass spectra of the most intense still unidentified peaks were manually interpreted and given an initial assignment as either PCDEs or OH-PCBs, as they share the same molecular formula $\mathrm{C}_{12} \mathrm{H}_{(10-\mathrm{x})} \mathrm{OCl}_{\mathrm{x}}$ (Figure 4.2C and D). When investigating the PCB congeners showing higher than expected values, interfering ions were observed in the spectra. For instance, Figure 4.2D shows additional ions in the spectrum of the PCB49 peak from the sample as compared to the mass spectra of pure PCB49 standard and trichlorinated-OH-PCB standard (4-hydroxy-2,2',5'trichlorobiphenyl); trichloro-diphenyl ether was not available at the time of analysis. 

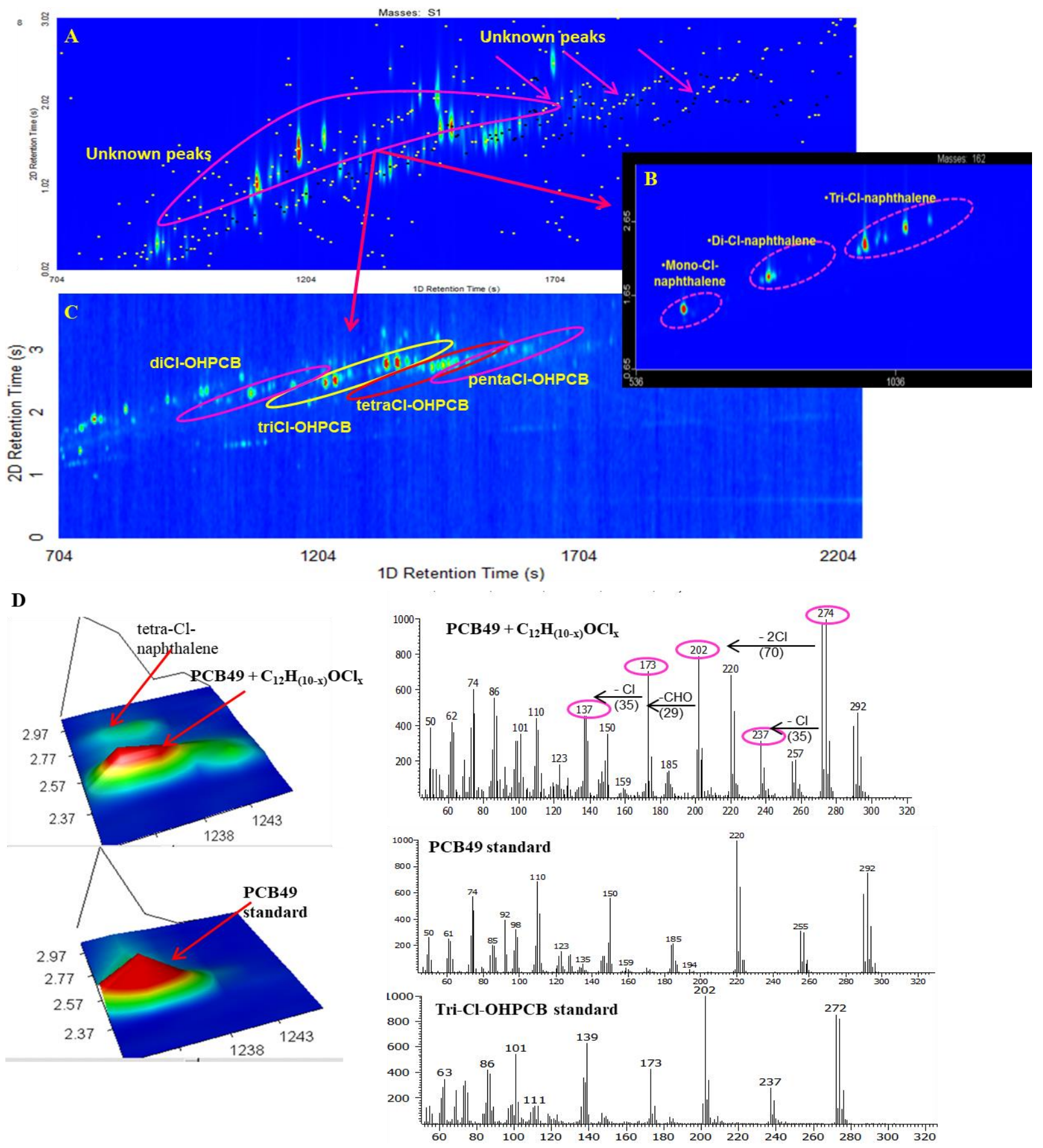

Figure 4.2 2D chromatograms and mass spectra for water sample \#2: A. GC $\times$ GC- $\mu$ ECD chromatogram; B. GC $\times$ GC-TOFMS chromatogram showing the PCNs identification; $\mathrm{C}$. GC $\times$ GC-TOFMS chromatogram showing the hydroxylated PCB identification, and D. PCB49 and $\mathrm{C}_{12} \mathrm{H}_{(10-\mathrm{x})} \mathrm{OCl}_{\mathrm{x}}$ interfering peak 
To further confirm which class of compounds was present in the samples, OH-PCB and PCDE standards were analyzed by GC×GC-TOFMS, and their mass spectra and retention times were compared to those acquired from the water samples. As expected for PCDEs, the loss of two chlorines from the molecular ion was responsible for the most intense peak, a pattern not observed for most of the compounds of interest. When the mass spectra of the unknown peaks were compared to those of $\mathrm{OH}-\mathrm{PCB}$, they showed closer similarities. In addition, one of the compounds matched both the retention times and the mass spectrum of the OH-PCB18 standard. To confirm that $\mathrm{OH}-\mathrm{PCBs}$ were indeed the compounds present in the water samples, additional derivatization with diazomethane was completed for the samples as well as for all the OH-PCB standards. The indication of complete derivatization reaction was that the yellow color still persisted after one hour. Figure 4.3A and B show the selected ion chromatograms for the tri-chlorinated and tetrachlorinated compounds in the water sample \#2, before and after derivatization. Similarly, the diand penta-chlorinated suspected $\mathrm{OH}-\mathrm{PCB}$ peaks were converted to their derivatives. All the identified peaks having the molecular formula $\mathrm{C}_{12} \mathrm{H}_{(10-\mathrm{x})} \mathrm{OCl}_{\mathrm{x}}$ were not found in the treated sample as they were derivatized to their methoxylated form (MeO-PCB). These findings confirmed that $\mathrm{OH}-\mathrm{PCB}$ were indeed the unknown compounds present in the samples. Despite the fact the $\mathrm{GC} \times \mathrm{GC}-\mu \mathrm{ECD}$ chromatograms revealed the presence of other unknown bands of compounds that eluted very close to the hexa- to octa- PCB retention times (Figure 4.2A - bands marked as "unknown peaks"), OH-PCBs with chlorination levels higher than penta- could not be confirmed by TOFMS. Based on previous studies that reported hexa- to octa-chlorinated OH-PCBs in surface water [125], we presumed that these bands, detected by $\mu \mathrm{ECD}$, were similar compounds with concentration levels lower than the TOFMS detection limits. 


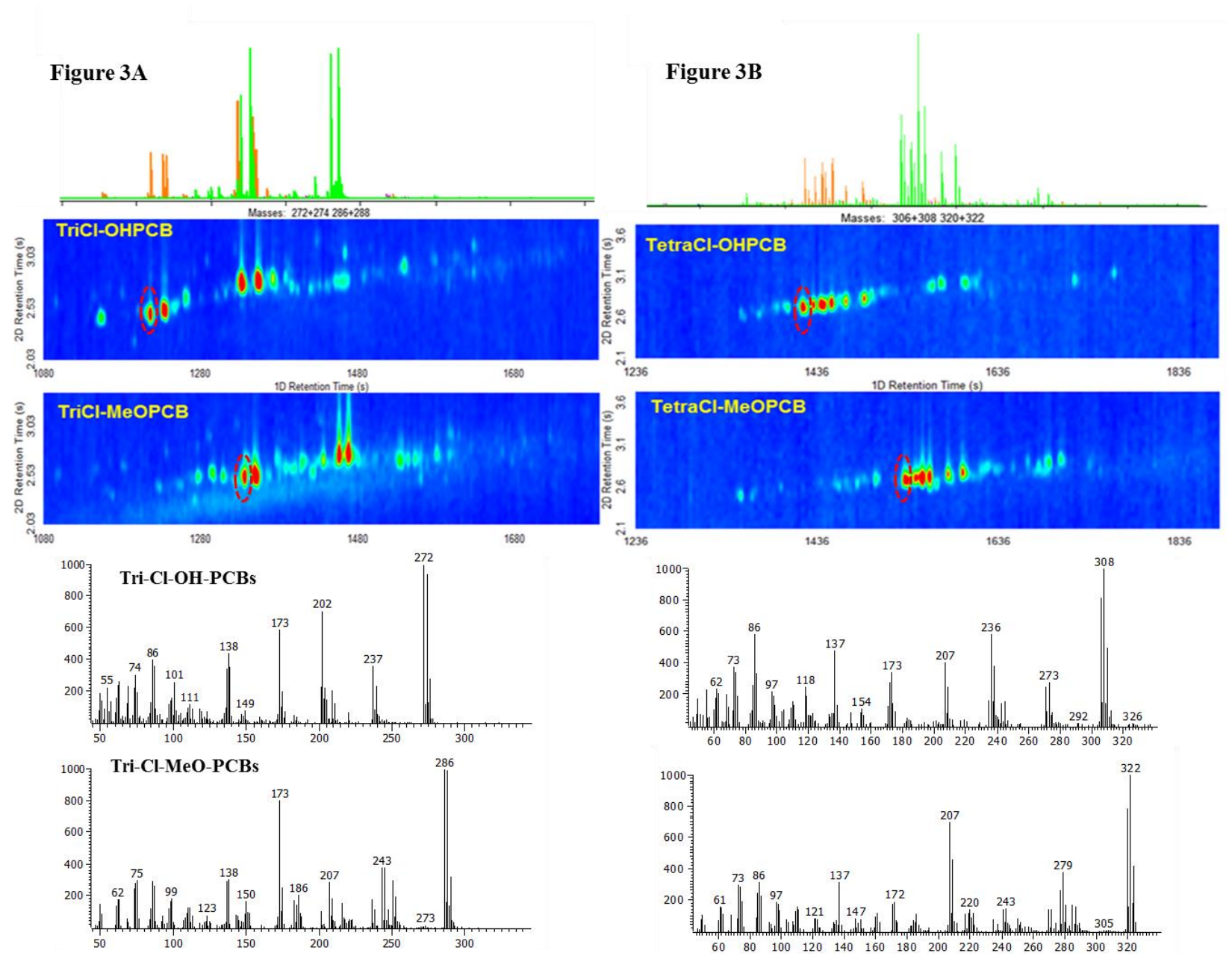

Figure 4.3. Selected ion chromatograms for: A. tri-chlorinated, and B. tetra-chlorinated compounds in the water sample \#2, before and after derivatization.

The TOFMS analyses confirmed the analytical interferences in the original GC $\times$ GC $-\mu \mathrm{ECD}$ method used, which explained the difficulties in matching the sample congener profiles with a mixed Aroclor profile. The samples were found to contain high concentrations of hydroxylated PCBs, which were interfering with some of the targeted PCB congeners, in particular congeners 49, 74, 84, 90/101 and 99. Despite the analytical interference of OH-PCBs and PCNs in the reporting of PCB concentrations, the PCB congener profiles were still consistent with a high 
proportion of Aroclor 1242, followed by Aroclor 1254. The presence of free-phase product and the very high concentrations of a mixture of other chlorinated compounds at the sampling location may have played a role in variable local fate and transport processes. OH-PCBs were not found in sample \#3 unlike the other two samples collected on-site, potentially due to the lack of free-phase product at this particular sampling location.

In addition to OH-PCBs, PCNs were also detected by GC×GC-TOFMS (Figure 4.2B) and interfered with some lower chlorinated PCBs (e.g. PCB28/PCB31). Finding PCNs in these samples helped track down the suspected PCN contamination source in the area.

\subsubsection{Semi-quantitative analysis of OH-PCB}

Both $\mathrm{GC} \times \mathrm{GC}$ systems ( $\mu \mathrm{ECD}$ and TOFMS) were multilevel calibrated using the ten purchased OH-PCB standards with different chlorination levels. As the number of available standards for this initial study was limited, a semi-quantitative approach was employed. The GC $\times$ GC-TOFMS system was used to semi-quantify the OH-PCB detected, while the GC $\times$ GC$\mu \mathrm{ECD}$ system was used for the recovery tests and the initial identification.

The GC $\times$ GC-TOFMS 2D chromatograms were rigorously inspected and all the peaks suspected to be OH-PCBs were checked before and after derivatization. OH-PCBs were identified in samples by comparing their mass spectra / fragmentation patterns with those of their corresponding chlorination level calibration standards. Each confirmed OH-PCB peak was then semi-quantified against its chlorination level equivalent, and the data were reported as the sum of di-, tri- and tetra-OH-PCBs (Table 4.1). Table 4.1 also shows the number of congeners identified for each homologue group, from dichloro- to pentachloro-OH-PCBs. OH-PCB with higher chlorination were not detected in any of the samples by GC×GC-TOFMS. Individual congener 
identification was not possible at this point of the study, except for OH-PCB18 which had matching retention times $\left({ }^{1} \mathrm{D}\right.$ and $\left.{ }^{2} \mathrm{D}\right)$ and mass spectrum. The results ranging from 33 to $50 \mathrm{ng} / \mathrm{L}$ for di-chlorinated, 150 to $1115 \mathrm{ng} / \mathrm{L}$ for tri-chlorinated, 150 to $882 \mathrm{ng} / \mathrm{L}$ for tetra-chlorinated and 73 to $89 \mathrm{ng} / \mathrm{L}$ for penta-chlorinated-OH-PCBs showed that the OH-PCB levels found in sample \#2 were several orders-of-magnitude higher than previously reported OH-PCB concentrations found in water samples from another study [125]. When compared to the total PCB concentration data, the ratio of OH-PCBs to total PCBs concentration was $7 \%$ for sample \#1 and $28 \%$ for sample \#2. Although it has been suggested that some OH-PCB presence should be expected in PCBcontaminated sediment due to $\mathrm{OH}-\mathrm{PCB}$ contamination in the originating Aroclor [132], what represents OH-PCB “Aroclor background" contamination relative to active or ongoing formation is unknown. Total $\mathrm{PCB} / \mathrm{OH}-\mathrm{PCB}$ ratios are likely to differ between sediment and water, as $\mathrm{OH}-$ PCBs are more water soluble than their parent PCB [121], which may explain higher OH-PCB to PCB ratios in water relative to sediment. Based on these findings, it is important to assess the presence of $\mathrm{OH}-\mathrm{PCB}$ when testing samples from known $\mathrm{PCB}$ contaminated sites, as $\mathrm{OH}-\mathrm{PCBs}$ might be more readily bio-available, more mobile and could have a large contribution towards the overall toxicity.

Table 4.1 Semi-quantitation of OH-PCBs for the storm sewer overflow water samples

\begin{tabular}{|l|c|c|c|c|c|}
\hline \multirow{2}{*}{ Sum of OH-PCB } & \multicolumn{2}{|c|}{ Sample \#1 } & \multicolumn{2}{c|}{ Sample \#2 } & Sample \#3 \\
\cline { 2 - 6 } & $\begin{array}{c}\text { \# of } \\
\text { Peaks }\end{array}$ & $\begin{array}{c}\text { Concentration } \\
(\mathrm{ng} / \mathrm{L})\end{array}$ & $\begin{array}{c}\text { \# of } \\
\text { Peaks }\end{array}$ & $\begin{array}{c}\text { Concentration } \\
(\mathrm{ng} / \mathrm{L})\end{array}$ & ND \\
\hline Dichloro-OH-PCB & 6 & 33 & 6 & 50 & ND \\
\hline Trichloro-OH-PCB & 13 & 150 & 20 & 1115 & ND \\
\hline Tetrachloro-OH-PCB & 16 & 155 & 20 & 882 & ND \\
\hline Pentachloro-OH-PCB & 10 & 89 & 15 & 73 & ND \\
\hline Total OH-PCB & 45 & 427 & 61 & 2119 & $\begin{array}{c}\text { Not } \\
\text { detected }\end{array}$ \\
\hline PCB total & \multicolumn{2}{|c|}{$6300 \mathrm{ng} / \mathrm{L}$} & \multicolumn{2}{|c|}{$7600 \mathrm{ng} / \mathrm{L}$} & $900 \mathrm{ng} / \mathrm{L}$ \\
\hline
\end{tabular}




\subsubsection{Recovery tests for OH-PCB}

The sample preparation procedures employed for the water samples were developed for the analysis of PCB congeners, OCs and chlorobenzenes. They were not optimized for OH-PCBs as they were not expected to be detected in any of the samples. Since the entire collected volume was extracted for all three water samples $(800 \mathrm{~mL}$ each) and more samples were not available for re-extraction with a proper procedure, recovery studies were performed to assess the sample preparation efficiency as employed at the time. The extraction and clean-up procedures were evaluated independently for the recoveries of the ten OH-PCBs with different chlorination levels and $-\mathrm{OH}$ positions by passing water blanks spiked with $200 \mu \mathrm{L}$ of the $\mathrm{OH}-\mathrm{PCB}$ spiking solution (final concentration of $200-1000 \mathrm{ng} / \mathrm{L}$ ) through the SPE extraction disks and clean-up cartridges ( $1 \mathrm{~g}$ silica), see Section 4.2.2. All the data for the recovery tests were obtained from GC $\times \mathrm{GC}$ $\mu E C D$ analyses. The results showed that approximately $45 \%$ of the di- to tetra-chlorinated $\mathrm{OH}-$ PCBs, and only $2 \%$ of the penta-chlorinated congeners, were recovered by using the HLB-M extraction procedure. Hexa- and hepta-chlorinated congeners were not recovered from the HLBM disk (Table 4.2). Similarly, the clean-up procedure using $1 \mathrm{~g}$ silica cartridges (see Section 4.2.2) resulted in recoveries shown in Table 4.2: $40 \%$ of the dichloro- congener were recovered; trichloro- and tetrachlo- congeners were retained on the cartridge, while the recoveries of pentachloro- congeners varied. Due to the recovery variations observed for the two different pentachloro- congeners during these tests, we suspected that the retention of the OH-PCBs on HLB disks and on silica cartridges depended on the molecule configuration (chlorine and $\mathrm{OH}$ - position). The presence of OH-PCBs in the final "real-sample" water extracts was believed to be due to a combination of factors: their presence at high levels in the samples and their retention on HLB disks and elution from the silica cartridges could be congener-specific. 
Table 4.2 Recovery tests for OH-PCBs for the extraction and clean-up procedures

\begin{tabular}{|l|c|c|c|}
\hline \multicolumn{1}{|c|}{ OH-PCB $(\mathbf{p g} / \boldsymbol{\mu L})$} & $\begin{array}{c}\text { Extraction } \\
\% \text { Rec. }\end{array}$ & $\begin{array}{c}\text { 1 g Silica } \\
\text { Clean-up } \\
\% \text { Rec. }\end{array}$ & $\begin{array}{c}\text { 5 g Florisil } \\
\text { Clean-up } \\
\% \text { Rec. }\end{array}$ \\
\hline 2-OH-2',3'-dichlorobiphenyl & 45.9 & 39.8 & 21.4 \\
\hline 4-OH-2,2',5'-trichlorobiphenyl & 47.4 & 1.9 & 1.0 \\
\hline 4-OH-2',3',4',5'-tetrachlorobiphenyl & 44.8 & 1.9 & 1.1 \\
\hline 3-OH-2',3',4',5,5'-pentachlorobiphenyl & 2.1 & 83.8 & 1.1 \\
\hline 3-OH-2,3',4,4',5-pentachlorobiphenyl & ND & 24.4 & ND \\
\hline 4-OH- 2,2',3,4',5,5'-Hexachlorobiphenyl & ND & 25.6 & ND \\
\hline $\begin{array}{l}\text { 4'-OH-2,2',3,3',4,5,5'-heptachlorobiphenyl / } \\
\text { 3'-OH-2,2',3,4,4',5,5'-heptachlorobiphenyl }\end{array}$ & ND & 63.6 & 2.3 \\
\hline
\end{tabular}

Finding the $\mathrm{OH}-\mathrm{PCB}$ in these water extracts when using $1 \mathrm{~g}$ silica cartridges also helped improve the clean-up procedure for the target compounds for the routine GC $\times \mathrm{GC}-\mu \mathrm{ECD}$ method. Silica cartridges $(5 \mathrm{~g})$ and Florisil cartridges ( $1 \mathrm{~g}$ and $5 \mathrm{~g}$ ) were tested as alternatives to remove these interfering compounds. When $5 \mathrm{~g}$ silica cartridges were used instead of the $1 \mathrm{~g}$ ones, the recoveries for some of the targeted organochlorine pesticides were very low $(<50 \%)$ and a more polar solvent mix (50\% DCM in hexane) was required to elute them, also along with more background interferences. Based on the recovery tests for the target compounds as well as $\mathrm{OH}$ PCBs, the $5 \mathrm{~g}$ Florisil cartridges were selected (see Table 4.2) as an alternative clean-up procedure. Recoveries for $\mathrm{PCBc} / \mathrm{OC} / \mathrm{CB}$ s ranged from $80 \%$ to $110 \%$, while only di-chlorinated OH-PCBs partially broke through $(21.4 \%)$. It is recommended that $5 \mathrm{~g}$ Florisil cartridges be used in future for the clean-up of extracts of water samples collected from suspected PCB contaminated sites, or when interfering peaks eluting close to PCBs are observed in the $2 \mathrm{D}$ GC $\times$ GC- $\mu$ ECD chromatograms. 


\subsection{Conclusions}

Often overlooked, metabolites of "historical" persistent organic pollutants such as PCBs should be included in either targeted or screening routine methods, as their toxic potential can exceed that of their parent compounds. By using a technique such as GC $\times$ GC- $\mu \mathrm{ECD}$ for routine analysis, a targeted method with capabilities for non-targeted analysis, the identification of $\mathrm{OH}-$ PCBs and other non-targeted halogenated contaminants such as PCNs present in the environmental samples became possible. Dichloro- to pentachloro- OH-PCB were detected in storm sewer water along with PCBs, at concentrations well in excess of those previously reported. This is of high environmental significance, as these toxic compounds in environmental matrices can easily be transported and might become bioavailable for uptake to different organisms. More studies are needed to assess the presence of OH-PCBs at known PCB contaminated sites and their distribution in different environmental matrices. The commercially available OH-PCB standards were purchased, and new targeted/non-targeted GC $\times \mathrm{GC}$ as well as GC-MS/MS methods are being developed. It is important to emphasize the importance of expanding the list of target contaminants in routine analysis of environmental samples and the need for more studies to assess the continuous exposure of the organisms/environment to these metabolites, not only to the legacy compounds. 


\section{Chapter 5}

\section{The Analysis of Polychlorinated Diphenyl Ethers in Sediments by Comprehensive Two-Dimensional Gas Chromatography with Micro-electron Capture Detection and Time-of-flight Mass Spectrometry ${ }^{v}$}

Polychlorinated diphenyl ethers (PCDE) are a group of halogenated organic contaminants consisting of 209 isomers with structure and properties very similar to those of polychlorinated biphenyls (PCBs), dibenzo-p-dioxins (PCDDs), dibenzofurans (PCDFs) and PCNs (polychlorinated naphthalenes). These classes of compounds are ubiquitous in the environment, stable, can biomagnify and are known to be toxic to living organisms [137-138]. Their physical and chemical properties are not very well known; vapour pressures, water solubility and noctanol/water partition coefficients $\left(\mathrm{k}_{\mathrm{ow}}\right)$ were determined by using chromatographic methods. The calculated and predicted $\log \mathrm{k}_{\mathrm{ow}}$ values range from 4.45 to 8.16 , and their water solubilities $(-\log \mathrm{S}$; mol/L) range between 4.21 and 12.95 [226]. Having similar properties to PCBs and being more water soluble than them, PCDEs are more bioavailable and have potential for bioaccumulation.

PCDEs were identified as by-products of chlorinated phenols and chlorinated phenoxyacetic acids formulations [138,139-140,227], at wood waste sites, in fly ash and in municipal incinerator wastes [141-142,228-229]. Chlorophenols were extensively used as herbicides and in wood preserving formulations, and their presence is ubiquitous in the environment. Other PCDE derivatives such as the herbicide nitrofen (nitro-PCDE) and the antimicrobial agent Triclosan

\footnotetext{
${ }^{\mathrm{v}}$ This chapter is based on the manuscript by A.M. Muscalu, T. Górecki, E.J. Reiner and D. Morse, "The Analysis of Polychlorinated Diphenyl Ethers in Sediments by Comprehensive Two-Dimensional Gas Chromatography with Micro-electron Capture Detection and Time-of-flight Mass Spectrometry", awaiting internal approval to release the data
} 
(hydroxylated-PCDE) were used and could be potential sources of PCDEs [139]. Similar to PCBs, PCDEs were used in different applications: as hydraulic fluids, electric insulators, flame retardants, lubricants and plasticizers, pesticides and synergistic pesticides [137-138]. As their production has been much smaller than that of PCBs. and both production and sales decreased in the 1970s, it is believed that the widespread environmental occurrence of PCDEs is mainly due to their presence at high levels $(100-1000 \mathrm{mg} / \mathrm{kg}$ [227]) in the technical chlorophenols formulations. These compounds have been reported in many environmental matrices, including biota samples [144146,230-231], water [232-233] and sediments [230-232,234].

Summarized in several published reviews, the toxicological studies on PCDEs showed that they can induce adverse reactions in living organisms; however, their ecotoxicological effects are unknown [137-140,227]. PCDEs can affect the oxidative status and metal homeostasis of the liver in mice [235], can have immunosuppressive effects in rats [227], and can result in chloracne and liver damage [138]. The lower chlorinated PCDEs exerted acute toxicity in fish, while the tetra- to deca-chlorinated CDEs increased the levels of cytochrome P-450 or increased monooxygenase activity in rats, similarly to PCBs [227]. The acute toxicity has been found to be dependent on the number and position of chlorine atoms in the molecule. There is not much data on carcinogenicity of these compounds. Even though PCDEs are not believed to be as highly toxic as other halogenated organics according to limited toxicology studies, they might be environmentally significant as they are possible precursors to toxic PCDDs and PCDFs [137-138,227,236-237]. Similarly to PCBs, the biological conversion of PCDEs to toxic metabolites must also be taken into account when assessing their overall toxicological effects.

The analysis of PCDEs is very complex, as fractionation of the extracts is required prior to instrumental analysis to separate these compounds from other halogenated compound classes (e.g. 
PCDD and PCDFs) that are present in the samples at the same time. There are no validated methods for the analysis of PCDEs; however, approaches similar to those used for PCB, PCDD and PCDF analyses are applied. Analytical methods employing GC coupled with different detectors were developed for the analysis of PCDE. Due to the lack of commercially available analytical standards, identification and quantitation of PCDEs are difficult. GC-ECD [238] was used in some studies, but GC coupled with both low resolution [144-146,239] and high resolution [142,147] MS operated in electron impact (EI) mode were the methods of choice. EI mass spectra of most PCDEs have an abundant molecular ion $\left(\mathrm{M}^{++}\right)$and a more abundant $\mathrm{M}^{++}-2 \mathrm{Cl}$ fragment that is mostly used in SIM analyses. Both external calibration and, for more accuracy and precision, isotope dilution, were used for quantification. The lack of commercially available labeled internal standards was the limiting factor for developing the isotope dilution methods [138].

Comprehensive two-dimensional gas chromatography is known to be one of the most powerful techniques for the separation of complex environmental samples, especially for the analysis of compound groups that contain congeners or homologues like polychlorinated biphenyl congeners (PCBs). The enhanced selectivity of $\mathrm{GC} \times \mathrm{GC}$ enables the less selective micro electron capture detector $(\mu \mathrm{ECD})$ to be an ideal tool for routine target compound analysis, and also allows the assessment of the presence of non-target contaminants. Coupled with different detectors ( $\mu \mathrm{ECD}$, TOFMS, HRTOMF), GC $\times \mathrm{GC}$ was used to assess the separation of different classes of halogenated contaminants and successfully applied for the identification and quantitation of POPs in different applications [2,48,240-241].

This study was focused on developing a quantitative method for the analysis of polychlorinated diphenyl ethers in sediment core samples by using comprehensive twodimensional gas chromatography. For this purpose, recovery tests and the preliminary assessments 
of the presence of PCDEs in samples were initially performed by $\mathrm{GC} \times \mathrm{GC}-\mu \mathrm{ECD}$, followed by GC $\times$ GC-TOFMS for confirmation and quantitation. In addition, we wanted to emphasize the importance of using a technique such as $\mathrm{GC} \times \mathrm{GC}-\mu \mathrm{ECD}$ for routine analysis to further identify non-targeted halogenated contaminants present in environmental samples.

\subsection{Materials and Methods}

\subsubsection{Standards}

For this study, a total of eight native PCDE standards and seven isotopically-labelled standards were purchased from Wellington Laboratories Inc. (ON, Canada). To obtain mass spectral confirmation and assess the chromatographic separation, the standards were selected based on different chlorination levels ranging from mono- to deca-chlorinated PCDEs: 4chlorodiphenyl ether (PCDE 3), 4,4'-dichlorodiphenyl ether (PCDE 15), 2,4,4'-trichlorodiphenyl ether (PCDE 28), 2,4,4',5-tetrachlorodiphenyl ether (PCDE 74), 3,3',4,4'-tetrachlorodiphenyl ether (PCDE 77), 2,2',4,4',5-pentachlorodiphenyl ether (PCDE 99), 2,2',3,4,4',5,5'heptachlorodiphenyl ether (PCDE 180) and decachlorodiphenyl ether (PCDE 209). Hexachloroand nonachlorodiphenyl ether standards were not available for purchase at the time of analysis. The following ${ }^{13} \mathrm{C}$-PCDE standards were acquired for quantitation and retention times reference purposes: 4-chloro $\left[{ }^{13} \mathrm{C}_{12}\right]$ diphenyl ether (MCDE 3), 3,4-dichloro $\left[{ }^{13} \mathrm{C}_{12}\right]$ diphenyl ether (MCDE 12), 3,4,4'-trichloro $\left[{ }^{13} \mathrm{C}_{12}\right]$ diphenyl ether (MCDE 37), 2,3,4,5-tetrachloro $\left[{ }^{13} \mathrm{C}_{12}\right]$ diphenyl ether (MCDE 61), 2,2',3,4,5-pentachloro $\left[{ }^{13} \mathrm{C}_{12}\right]$ diphenyl ether (MCDE 86), 2,2',3,4,5,5'hexachloro $\left[{ }^{13} \mathrm{C}_{12}\right]$ diphenyl ether (MCDE 141) and 2,2',3,4,4',5,5'-heptachloro $\left[{ }^{13} \mathrm{C}_{12}\right]$ diphenyl ether (MCDE 180). 
Analyte identification and the development of multi-level calibration curves were carried out using both GC $\times$ GC- $\mu$ ECD and GC $\times$ GC-TOFMS. This included the determination of the linearity of the instrument response, as well as evaluation of different calibration procedures (external versus internal standard calibrations for GC×GC-TOFMS, where the labelled PCDEs were used as internal standards.). For instrument calibration, five-level standard solutions ranging from 5 to $500 \mathrm{ng} / \mathrm{mL}$ for $\mathrm{GC} \times \mathrm{GC}-\mu \mathrm{ECD}$ and from 10 to $1000 \mathrm{ng} / \mathrm{mL}$ for the TOFMS were prepared in iso-

octane. An MCDE standard mixture solution was prepared in iso-octane and was added to all multi-level calibration standards and samples prior to $\mathrm{GC} \times \mathrm{GC}-\mathrm{TOFMS}$ at a final concentration of $125 \mathrm{ng} / \mathrm{mL}$. In addition, a spiking solution of PCDE standards containing all eight congeners was prepared in acetone with a final concentration of $5 \mu \mathrm{g} / \mathrm{mL}$ (resulting in a final concentration of 25

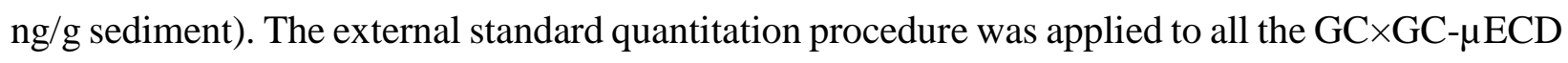
data. The ${ }^{13} \mathrm{C}-\mathrm{PCDE}$ standards were used as internal standards for both the quantitation and semiquantitation procedures applied to the quality control samples and samples analyzed by GC×GCTOFMS.

In-house prepared diazomethane was used to derivatize the sediment sample extracts to further confirm the identity of PCDEs.

\subsubsection{Sample Collection and Sample Preparation}

Eleven core sediment samples were collected in 2017 from Whitby Harbour, ON, Canada, and further divided into 37 samples based on the core depths. These samples were collected from an area previously known to be contaminated with PCDEs [230,242]. The details about the core samples and their location are not presented here as it is beyond the scope of this research. 
The whole sample was air-dried, crushed and sieved ( $2 \mathrm{~mm}$ sieve). Further, a portion of the sediment sample (5 g) was extracted using accelerated solvent extraction (ASE, Dionex ${ }^{\mathrm{TM}}$ ASE $^{\mathrm{TM}}$ 350, Thermo Scientific, Bannockburn IL, USA). The ASE conditions were as follows: one cycle extraction at $100^{\circ} \mathrm{C}$, heat time $5 \mathrm{~min}$., purge time $90 \mathrm{~s}$, flush volume $60 \%$, extraction solvent 25 $\%$ dichloromethane $/ 75 \%$ hexane $(\mathrm{v} / \mathrm{v})$. The resulting extract was subjected to a single stage silica cartridge cleanup procedure and copper treatment to remove sulfur interferences prior to instrumental analysis [27-28]. No fractionation of extracts was required, which resulted in significant time and analysis costs savings.

\subsubsection{Instrumental Analysis}

The two GC×GC systems (LECO Corp., St Joseph, MI, USA) used for this study consisted of an Agilent 6890 gas chromatograph (Agilent Technologies, Santa Clara, CA, USA) equipped with a split/splitless injector, a cryogen-free modulator (LECO), and a $\mu$ ECD or a TOFMS detector, respectively. The following chromatographic column combination was used with GC $\times$ GC- $\mu$ ECD: 30 m, 0.25 mm i.d., $0.25 \mu \mathrm{m}$ film thickness DB-1 (100 \% dimethylpolysiloxane) from J\&W Scientific (Folsom, CA, USA) as the first dimension column, $1.6 \mathrm{~m}, 0.18 \mathrm{~mm}$ i.d., 0.18 $\mu \mathrm{m}$ film thickness Rtx-PCB from Restek Corp. (Bellefonte, PA, U.S.A.) as the second dimension column, and a $0.3 \mathrm{~m}, 0.1 \mathrm{~mm}$ i.d. IP-Deact column (Restek Corp.) as the transfer line to the detector. The GC×GC-TOFMS system used a $60 \mathrm{~m}, 0.25 \mathrm{~mm}$ i.d., $0.25 \mu \mathrm{m}$ film thickness Rxi1MS (100\% dimethylpolysiloxane) from Restek Corp. (Bellefonte, PA, U.S.A.) as the first dimension column, followed by a $1.3 \mathrm{~m}, 0.25 \mathrm{~mm}$ i.d., $0.25 \mu \mathrm{m}$ film thickness Rtx-PCB (Restek Corp.) as the second dimension column. The temperature program for the primary oven started at $80{ }^{\circ} \mathrm{C}$ (hold for $2 \mathrm{~min}$ ) to $160{ }^{\circ} \mathrm{C}$ at $10{ }^{\circ} \mathrm{C} / \mathrm{min}$, then to $280{ }^{\circ} \mathrm{C}$ at $4{ }^{\circ} \mathrm{C} / \mathrm{min}$ (hold for $7 \mathrm{~min}$ ). The 
secondary oven had a $35^{\circ} \mathrm{C}$ positive temperature offset to the first dimension oven. The modulation period was $4 \mathrm{~s}$, with a modulator temperature offset of $30^{\circ} \mathrm{C}$ to the first dimension oven. The injection volumes (splitless mode) of the final extract were $1 \mu \mathrm{L}$ for $\mathrm{GC} \times \mathrm{GC}-\mu \mathrm{ECD}$ and $2 \mu \mathrm{L}$ for GC $\times$ GC-TOFMS. The inlet temperature was $250{ }^{\circ} \mathrm{C}$, and a $4 \mathrm{~mm}$ i.d. gooseneck liner was used (Restek Corp.). Helium gas (Praxair, Mississauga, ON, Canada) with a purity of $99.999 \%$ was used as carrier gas at $1.2 \mathrm{~mL} / \mathrm{min}$ flow rate. The micro-ECD system was operated at $300{ }^{\circ} \mathrm{C}$ using data acquisition rate of $50 \mathrm{~Hz}$, with $5 \%$ methane in argon as the detector make-up gas at a flow rate of $150 \mathrm{~mL} / \mathrm{min}$. The transfer line to TOFMS was operated at $300{ }^{\circ} \mathrm{C}$ and the ion source temperature was set to $280^{\circ} \mathrm{C}$. ChromaTOF software (LECO Corp.) was used for data acquisition and processing for all analyses performed in this study.

\subsection{Results and Discussion}

\subsubsection{Chromatographic separations}

The PCDE standards were initially analyzed by GC $\times \mathrm{GC}-\mu \mathrm{ECD}$ to evaluate both the withinand between-class separation, as well as to assess the quantitation when using this technique. It was observed that PCDE retention times were very close to those of PCBs (Figure 5.1 A and B), as well as OH-PCBs (presented in Chapter 4). Since the GC $\times \mathrm{GC}-\mu \mathrm{ECD}$ is used routinely for the analysis of PCBs, OCs and chlorobenzenes in solid samples [27-28], this was an important finding as the analysts need to be aware of what can be the possible "interferences" observed in the chromatograms and carefully assess the patterns before reporting the data. In addition, as PCDEs and OH-PCBs share the same molecular formula $\left(\mathrm{C}_{12} \mathrm{H}_{(10-\mathrm{x})} \mathrm{OCl}_{\mathrm{x}}\right)$ and fragmentation patterns, and their retention times are very similar in the 2D chromatograms, extra attention needs to be paid as to not misidentify these compounds in either ECD or MS analyses. PCDEs were less retained in 
the 2D plane, thus separated from other classes of halogenated contaminants such as PCDDs, PCDFs, PCNs and BDEs. Being chromatographically separated from the PCDFs is important for MS analysis, as the most intense fragmentation ion $\mathrm{M}^{\cdot+}-70\left(\mathrm{M}^{\cdot+}-2 \mathrm{Cl}\right)$ for PCDEs is the same as the molecular ion of PCDFs [138], and is not recommended to be used for quantitation.

As initial screening, the sediment samples were analyzed by $\mathrm{GC} \times \mathrm{GC}-\mu \mathrm{ECD}$ to confirm that other compound classes beside PCBs, OCs and CBs were indeed present in the samples. A closer look at the GC $\times \mathrm{GC}-\mu \mathrm{ECD}$ chromatogram revealed the presence of other classes of halogenated contaminants that eluted very close to PCBs' retention times (Figure 5.1B). Micro-ECD, a very sensitive but not specific detector, could not provide further information for characterizing the unknown compounds; thus, the sediment sample extracts were further analyzed by GC $\times \mathrm{GC}$ TOFMS. Figure 5.1 C shows the 2D separation of the PCDEs in the two-dimensional plane for one of the three samples collected from the most contaminated sediment core. Similar to PCBs, they are separated based on the number of chlorines in the molecule, resulting in an ordered chromatogram which is very useful for data interpretation. 

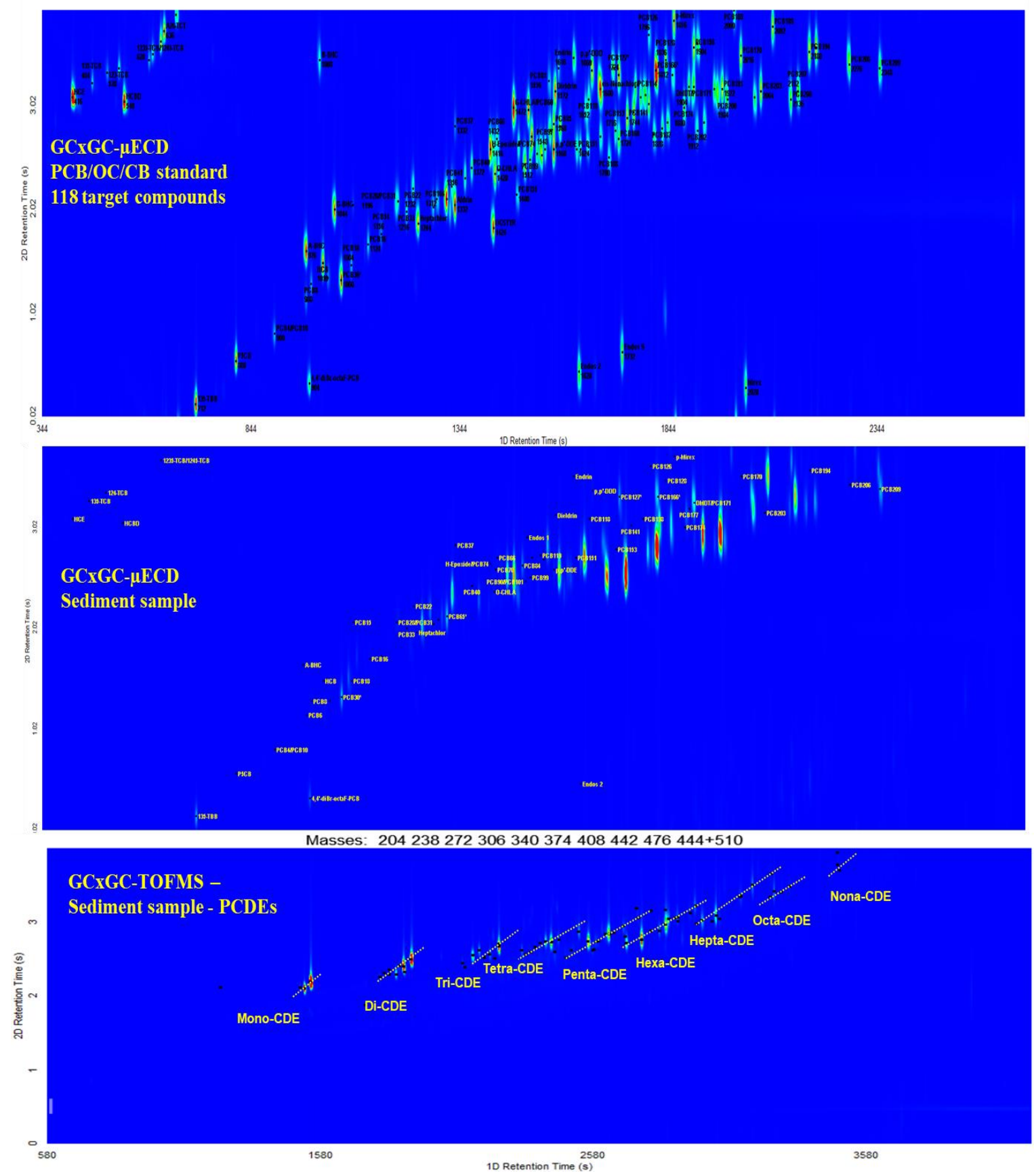

Figure 5.1 Two-dimensional chromatograms representing the separation of $\mathrm{A})$. PCB/OC/CB standard (118 target compounds [32]), B). PCDEs detected by GC $\times$ GC- $\mu$ ECD showing retention times similar to PCBs (PCBs - labelled in yellow, "unknowns" - not labelled) and C). The $\mathrm{GC} \times \mathrm{GC}-\mathrm{TOFMS}$ analysis of the sediment sample confirming the presence of PCDEs (selected ion monitoring). 


\subsubsection{Recovery tests for PCDEs}

PCDEs are very similar compounds to polychlorinated biphenyls (PCBs), and the same sample preparation procedure was tested (see Section 5.2 and references [27-28]). Thus, prior to processing the "real-life" sediment samples, recovery tests were performed separately for each step to evaluate extraction and clean-up efficiencies, as well as evaporation losses during the two evaporation steps. The sediment blank used for the spiked samples was previously analyzed and proven to be PCDE free. Two fortified sediment samples (50 ng/g for each PCDE congener) were passed through the extraction procedure, evaporated to $1 \mathrm{~mL}$ final volume, then analyzed by GC $\times$ GC- $\mu$ ECD. Similarly, two replicates of $1 \mathrm{~mL}$ iso-octane were spiked with PCDEs $(50 \mathrm{ng} / \mathrm{mL})$, then passed through $1 \mathrm{~g}$ silica cartridges, evaporated to $1 \mathrm{~mL}$ final volume and analyzed by $\mathrm{GC} \times \mathrm{GC}-\mu \mathrm{ECD}$. In addition, two replicates of fortified blank material per batch of sediment samples (two batches in total) went through the same sample preparation procedure along with the samples to monitor the recoveries for both native and labelled PCDEs. The recoveries for the extraction tests ranged from 97 to $111 \%$, while those for the clean-up tests were between 89 and $102 \%$ (Figure 5.2). The quantification procedure was based on external standard calibration for $\mu \mathrm{ECD}$ analyses. 


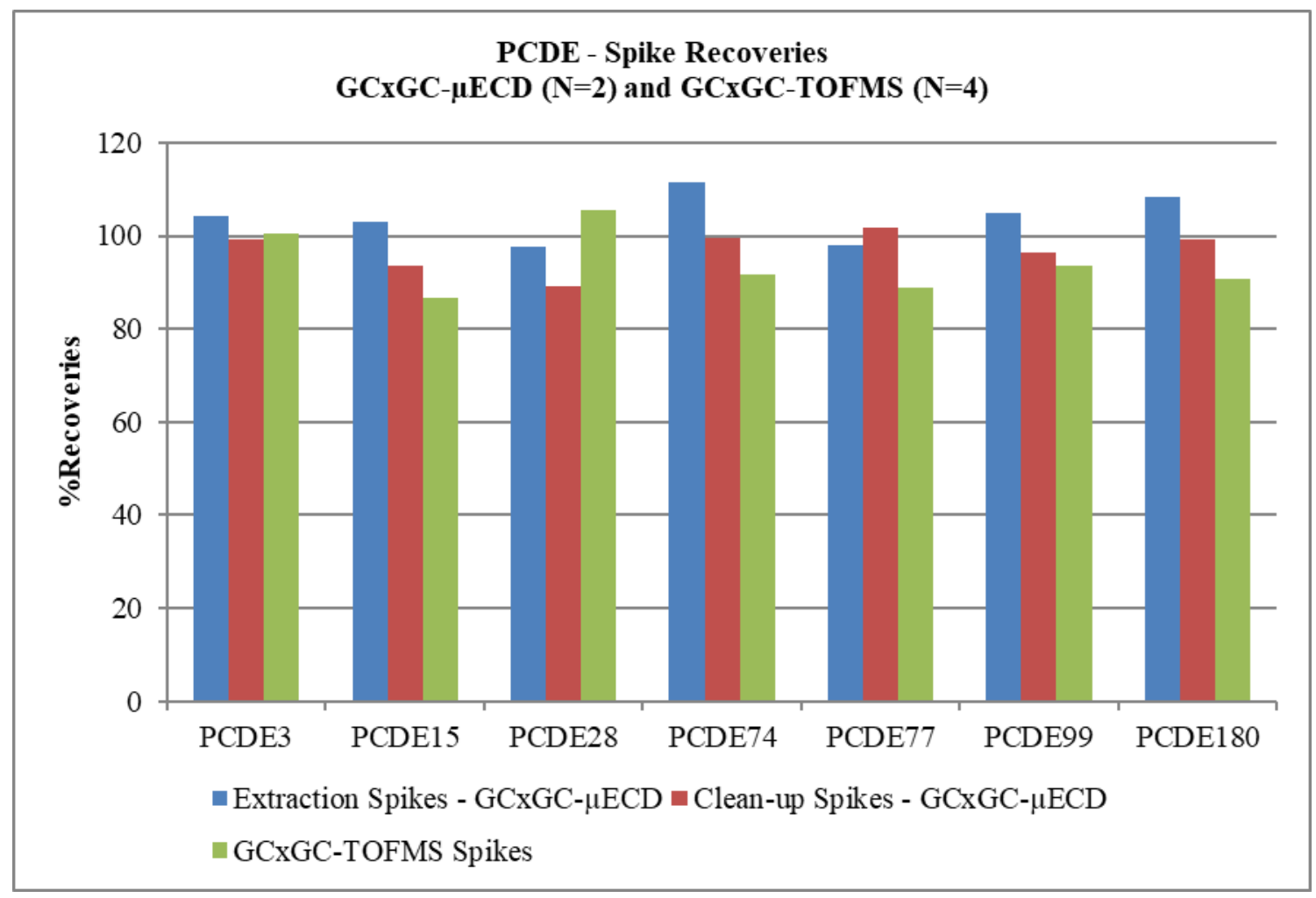

Figure 5.2 PCDE recovery tests for sample preparation procedure

The recoveries obtained for MCDE internal standards for the four spike replicates analyzed along with the samples ranged from 60 to $90 \%$, while for the native PCDEs were between 85 to $106 \%$ with internal standard correction (Figure 5.2). In addition, for some of the sediment samples, significant signal enhancements of up to $200 \%$ were observed for MCDE internal standards. Based on these results, to increase data accuracy, the internal standard calibration procedure was applied for all samples analyzed by TOFMS.

\subsubsection{Quantitative and semi-quantitative analysis of PCDEs in sediments samples}

Both GC $\times$ GC systems ( $\mu$ ECD and TOFMS) were multilevel-calibrated using the eight purchased PCDE standards with different chlorination levels. The GC $\times \mathrm{GC}-\mu \mathrm{ECD}$ system was 
external standard calibrated and was used to assess the recovery tests and the initial identification. As the number of available standards for this study was limited, both quantitative and semiquantitative approaches using internal standard $\left({ }^{13} \mathrm{C}\right.$-labelled PCDEs) calibration procedure were employed for GC×GC-TOFMS analyses. Multi-level calibrations were set-up for the PCDE standards having different degrees of chlorination, except for hexa- and octachlorinated congeners, which were not available at the time of analysis. The molecular ion and the fragment ion originating from the loss of $\mathrm{COCl}\left(\mathrm{M}^{++}-63\right)$ were used to quantitate mono-CDEs; the molecular ion and the fragment originating from the loss of two chlorines $\left(\mathrm{M}^{++}-70\right)$ were used for quantitating the rest of PCDEs. When the two tetra-chlorinated congeners, PCDE 74 and PCDE 77 were analyzed, it was observed that the most intense ion for the non-ortho congener (PCDE 77) was the molecular ion followed by the fragment ion originating from the loss of $\mathrm{COCl}$. Thus, for PCDE 77 and all the tetrachlorinated isomers identified in the sample that exhibited similar pattern, the molecular ion and $\mathrm{M}^{+}-63$ fragment were used for quantitation (Figure 5.3). 
PCDE-74 (2,4,4',5-TetraCl)

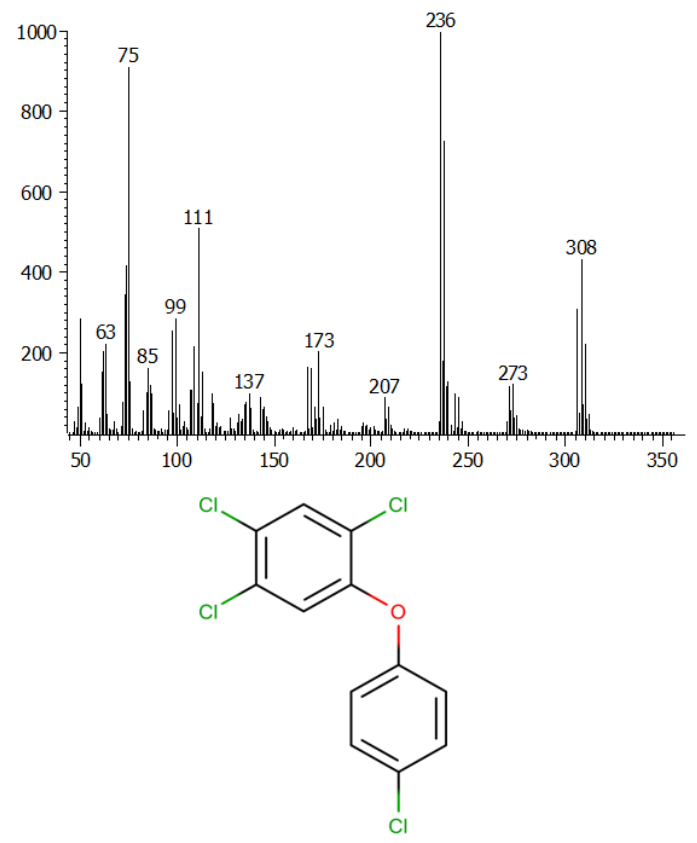

PCDE-77 (3,3',4,4'-TetraCl)

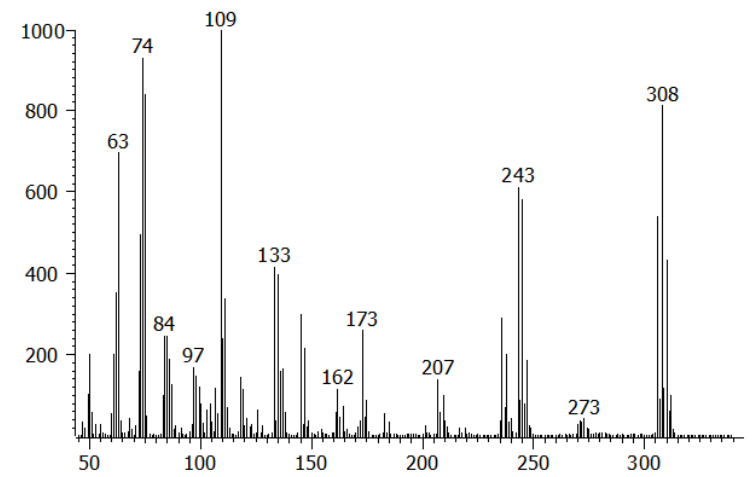

Figure 5.3 Mass spectra of two tetrachlorodiphenyl ethers: PCDE 74 and PCDE 77

The GCxGC-TOFMS 2D chromatograms were rigorously inspected and all the peaks suspected to be PCDEs were examined. Matching the retention times and mass spectra, seven of the eight targeted PCDEs ranging from mono- to nonachlorodiphenyl ethers were identified in the sediment samples and were quantitated directly. The decachlorinated congener was not detected in any of the 37 sediment extracts analyzed. To be confirmed as a PCDE, they had to have the correct molecular elemental composition, as well as exhibit the fragment ions originating from the loss of $\mathrm{Cl}_{2}$ and $\mathrm{COCl}$ for the molecular ion. As a result, a total of 68 PCDE peaks were identified in the most contaminated samples and further manually assigned and semi-quantified against the PCDE standard with the same chlorination level. Due to the lack of commercially available 
standards at the time of the analysis, the hexachlorinated PCDEs were semi-quantified against their pentachlorinated equivalent, and the octa- and nona-chlorinated PCDEs were semi-quantified against the heptachlorinated PCDE calibration curve. This may have resulted in a slight bias for these two congener groups. Also, along with the samples, quality control samples (matrix blanks and spikes) were analyzed to assess the analyte recoveries and instrument performance. Table 5.1 shows the data obtained for one sediment core sample divided into 3 sub-samples that presented high-levels of PCDEs. Similar to a previous study for samples collected from the same area [230], finding PCDEs at these levels was not surprising. However, one of the most important findings of the current study was the detection of mono- to tri-chlorinated congeners not previously reported. It was found that not only were they present at high levels, but their summed concentration accounted for more than $30 \%$ of the total PCDE value reported. This is environmentally significant, as these lower chlorinated PCDEs are more water soluble than the higher chlorinated ones and could be more bioavailable for the uptake by aquatic organisms. 
Table 5.1 Levels of PCDEs in a sediment sample analyzed by GC×GC-TOFMS

\begin{tabular}{|c|c|c|c|c|}
\hline \multirow[b]{2}{*}{ Total PCDE Calculations } & \multicolumn{3}{|c|}{ Amount \% } & \multirow[b]{2}{*}{$\begin{array}{c}\text { \# Peaks } \\
\text { Detected }\end{array}$} \\
\hline & $\begin{array}{c}\text { Sample } \\
\text { 6A }\end{array}$ & $\begin{array}{c}\text { Sample } \\
6 \mathrm{~B}\end{array}$ & $\begin{array}{c}\text { Sample } \\
\text { 6C }\end{array}$ & \\
\hline Total mono-Cl-PCDE & 16 & 19.2 & 10.1 & 3 \\
\hline Total di-Cl-PCDE & 23 & 18.8 & 26.8 & 8 \\
\hline Total tri-Cl-PCDE & 8.2 & 7.8 & 12.8 & 9 \\
\hline Total tetra-Cl-PCDE & 6.7 & 10.8 & 7.8 & 10 \\
\hline Total penta-Cl-PCDE & 10.9 & 10.7 & 17.6 & 13 \\
\hline Total hexa-Cl-PCDE & 19.0 & 20.9 & 18.7 & 11 \\
\hline Total hepta-Cl-PCDE & 13.0 & 10.2 & 6.1 & 8 \\
\hline Total octa-Cl-PCDE & 2.2 & 1.5 & ND & 5 \\
\hline Total nona-Cl-PCDE & 0.1 & ND & ND & 1 \\
\hline \multicolumn{4}{|c|}{ Amount ng/g } & $\begin{array}{l}\text { Total \# } \\
\text { Peaks }\end{array}$ \\
\hline Total PCDE (ng/g) & 8018 & 175 & 36 & 68 \\
\hline
\end{tabular}

Significant signal enhancements were observed for the ${ }^{13} \mathrm{C}$-labelled PCDEs in some of the sediment samples analyzed. When quantitating the PCDEs, the internal standard quantitation procedure was critical in these cases. Diluting the samples (1:10 dilution) was one way to minimize these enhancements; however, the low level PCDEs detected in the samples would be over-diluted and impossible to quantitate.

With the exception of the standards acquired for this study, individual congener identification was not possible at the time. The retention times for all the congeners detected (68 PCDE congeners), examples of mass spectra and the concentration levels for PCDEs in all 37 samples analyzed are presented in Appendix D. The PCDE congeners' distribution for the three samples collected from one of the most contaminated sediment cores is presented in Figure 5.4 (see also Table 5.1 for PCDE total values in the three samples). 

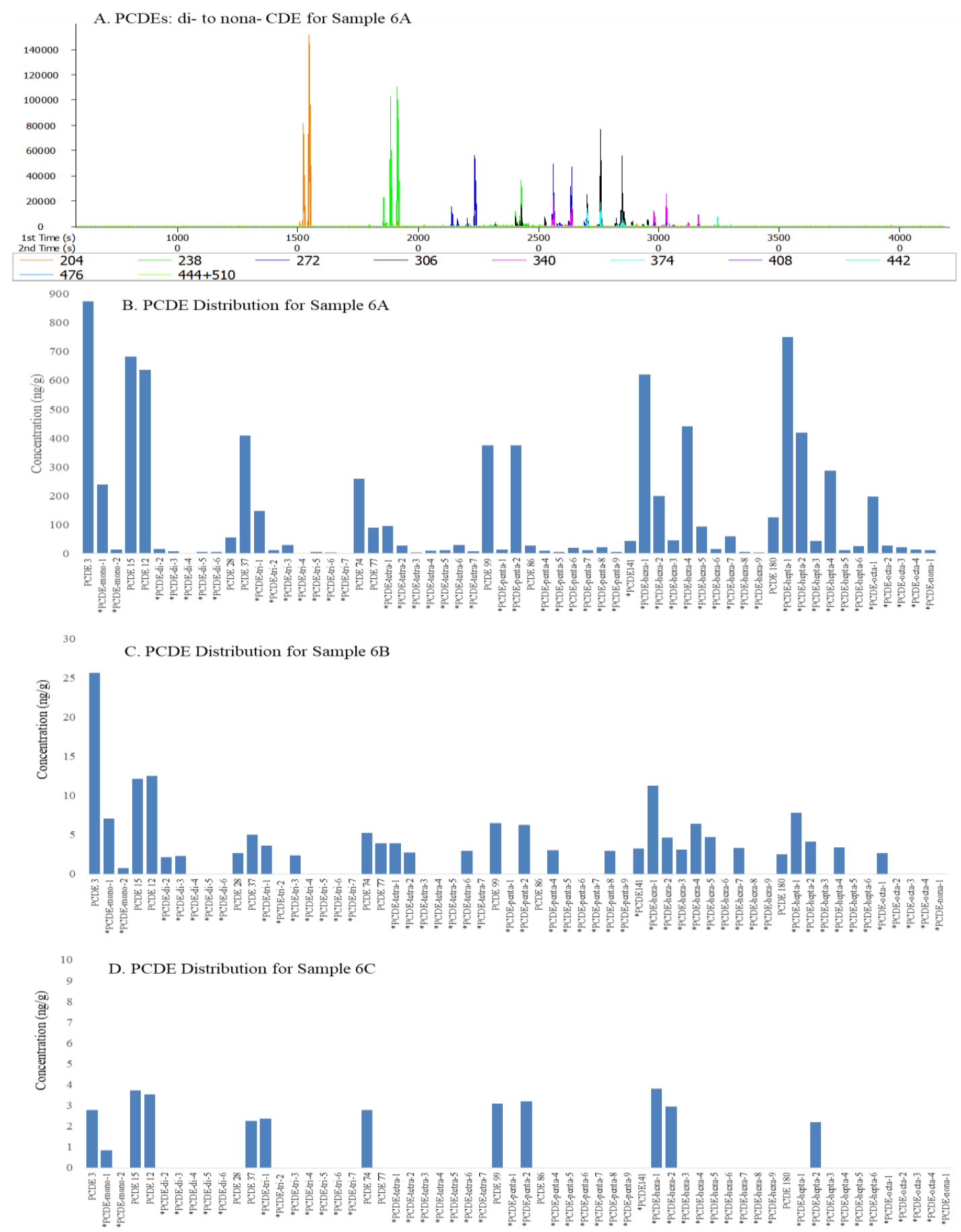

Figure 5.4 Sediment samples analyzed by GC×GC-TOFMS: A). Reconstructed 1D chromatogram for sample 6A for selected ions; B). to D). PCDE congeners' distribution in the three samples from one of the most contaminated sediment cores. 
To further confirm which class of compounds was present in the samples, PCDEs and / or OH-PCBs, derivatization was performed for each sediment core (one sample extract per sediment core). The indication of complete derivatization reaction was that the yellow color still persisted after one hour. The GC $\times$ GC-TOFMS analyses showed that no methoxylated compounds (MeOPCB) were formed during the derivatization step. These findings confirmed that PCDEs were indeed the unknown compounds present in the samples. It should be emphasized that even though not necessary for the PCDEs analysis, the derivatization is a very important step to confirm their

presence and not get them confused with OH-PCBs. Often overlooked, these two classes of compounds have the same elemental composition and similar mass spectral fragmentation patterns and cannot be distinguished from one another without additional confirmation tests. In addition, their retention times were very similar in the $2 \mathrm{D}$ chromatograms.

\subsection{Conclusion}

As the number of chemicals present in the environment is continuously growing, the use of $\mathrm{GC} \times \mathrm{GC}$ offers in-depth characterization of different components in complex mixtures and more information on the composition of different groups of xenobiotics. Its enhanced chromatographic resolution enabled improved data accuracy, provided fingerprinting information and identification compounds such as PCDEs that would normally be missed with the use of a one-dimensional target analysis. In this study, a method has been developed to screen for PCDEs by using GC $\times \mathrm{GC}-\mu \mathrm{ECD}$, then quantify them by GCxGC-TOFMS in environmental samples. This study reported mono- to trichlorinated PCDE congeners at high concentrations and as major contributors to the total amount of PCDE in the samples, not previously reported for samples collected from similar areas. In addition, this study emphasizes the importance of expanding the list of target contaminants in 
routine analysis of environmental samples and the need for more studies to assess the continuous exposure of the organisms/environment to these compounds or mixtures of compounds. Using a method such as $\mathrm{GC} \times \mathrm{GC}-\mu \mathrm{ECD}$ for routine analysis has the potential for initial screening of other halogenated contaminants present in the environmental samples. Also, this method can be used for triage: when other "unknown" peaks are observed in GC $\times \mathrm{GC}-\mu \mathrm{ECD}$ chromatograms, initial identification can be performed, then the samples can be re-directed to target validated mass spectrometry methods for more accurate analysis. This can be cost effective for an environmental laboratory, as the samples will not undergo expensive tests unless positive results are reported by ECD, a less costly test to analyze target compounds and assess non-target analytes. 


\section{Chapter 6 Final Conclusions}

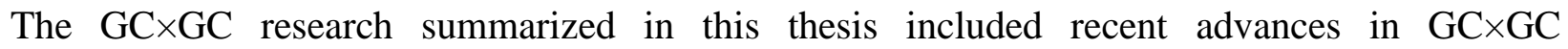
instrumentation, the development of $\mathrm{GC} \times \mathrm{GC}$ methods and their application for the analysis of environmental samples. The instrumentation and analytical methods presented proved highly valuable in the analysis of environmental samples.

The research presented in this thesis can be summarized as follows:

1. The performance of a new consumable-free single stage thermal modulator for $\mathrm{GC} \times \mathrm{GC}$ developed at the University of Waterloo was evaluated by comparing its performance to that of a commercially available $\mathrm{LN}_{2}$ modulator. The experimental data obtained was accurate and precise for the standards and reference material tested. Also, without any further optimization of the GC method and without using the secondary oven, two co-elutions found in the $\mathrm{LN}_{2}$ method were resolved when the newly developed modulator was used.

2. SCCPs, very complex mixture, were separated in distinct ordered bands by $\mathrm{GC} \times \mathrm{GC}-\mu \mathrm{ECD}$ pointing to group separation. Quantification procedures were developed by using ChromaTOF software and its Classification function combined with Excel to generate summary tables and calibration curves for different technical mixes. The method's performance was initially demonstrated on fortified sediment samples and further validated by participating in an inter-laboratory studies for the analysis of standards and sediment samples. The results showed recoveries between $75 \%$ to $95 \%$ and z-score values < 2 , demonstrating that the method is suitable for the analysis of SCCPs in soil/sediment samples. 
3. Using GC $\times$ GC- $\mu \mathrm{ECD}$ and GC $\times$ GC-TOFMS dichloro- to pentachloro- OH-PCB were detected and semi-quantitated in storm sewer water along with PCBs, at concentrations well in excess of those previously reported. "Unknown" peaks observed in the GCxGC- $\mu \mathrm{ECD}$ two dimensional chromatograms were further investigated by GC×GC coupled with time-of-flight mass spectrometry (TOFMS) and tentatively identified as OH-PCBs. Mass spectral data and further derivatization confirmed the presence of dichloro- to pentachloro-mono-OHPCBs. The discovery of OH-PCBs in the storm sewer water samples is of environmental significance since these compounds might have similar or higher toxicity than their parent compounds, yet they are not routinely monitored. In addition, other POPs such as PCNs were identified in the samples when using this technique.

4. The enhanced chromatographic resolution of $\mathrm{GC} \times \mathrm{GC}$ helped with improving data accuracy, providing fingerprinting information and identifying compounds such as PCDEs that would normally be missed with the use of a one-dimensional target analysis. Using a method such as $\mathrm{GC} \times \mathrm{GC}-\mu \mathrm{ECD}$ for routine analysis of targeted pollutants facilitated the initial screening of other halogenated contaminants present in the environmental samples and resulted in the detection of PCDEs in sediment samples. Following up the initial identification by GCxGC- $\mu$ ECD and confirmation by GCxGC-TOFMS of the observed "unknown" peaks, quantitative and semiquantitative approaches were applied for the analysis of polychlorinated diphenyl ethers. As a result, mono- to nona-chlorinated diphenyl ethers were detected and 
quantified in core sediment samples. Quantitation procedure by using GC×GCTOFMS was developed for PCDEs.

The following conclusions can be drawn from the results presented in this study:

1. The newly developed consumable free and cost-effective modulator provided accurate and precise results when compared to the commercially available $\mathrm{LN}_{2}$ modulator. This device was very easy to install and operate on any GC system making it a less expensive alternative for performing $\mathrm{GC} \times \mathrm{GC}$ separations. Furthermore, its flexibility gives new opportunities of performing comprehensive two-dimensional GC with other detectors that are fast enough to handle the narrow peaks generated by GCxGC.

2. The high separation power of $\mathrm{GC} \times \mathrm{GC}$ combined with the possibility of generating structured chromatograms made the identification of SCCPs in environmental samples possible. The novel approach for the quantification procedure developed with this method allowed the accurate quantitation for different technical mixes of SCCPs as well as "real" environmental samples. This is a valuable application as it generates not only qualitative data for the identification of different SCCPs mixtures by using a simple detector as ECD but also quantitative results. In addition, other classes of POPs can be simultaneousely identified and quantified within the same run.

3. By using a technique such as $\mathrm{GC} \times \mathrm{GC}-\mu \mathrm{ECD}$ for routine analysis, a targeted method with capabilities for non-targeted analysis, the discovery, identification and semiquantitation of $\mathrm{OH}-\mathrm{PCBs}$ and other non-targeted halogenated contaminants present 
in the environmental samples became possible. Often overlooked, metabolites of "historical" persistent organic pollutants such as PCBs should be included in either targeted or screening routine methods, as their toxic potential can exceed that of their parent compounds. This finding is of high environmental significance, as these toxic compounds in environmental matrices can easily be transported and might become bioavailable for uptake to different organisms. More studies are needed to assess the presence of OH-PCBs at known PCB contaminated sites and their distribution in different environmental matrices. In addition, the current findings emphasize that using a technique such as $\mathrm{GC} \times \mathrm{GC}-\mu \mathrm{ECD}$ for the routine testing of environmental samples is an excellent screening approach for detecting nontargeted contaminants.

4. This study reported for the first time mono- to trichlorinated PCDE congeners at high concentrations and as major contributors to the total amount of PCDE in the samples for the first time. This is environmentally significant as these lower chlorinated PCDEs are more water soluble, thus, more bioavailable for the uptake by the aquatic organisms.

The use of GC×GC offers in-depth characterization of different components in complex environmental mixtures and more information on the composition of different groups of xenobiotics. The analysis of environmental samples is very complex; the methods presented here proved to be efficient when multiple classes of compounds are present at the same time. The $\mathrm{GC} \times \mathrm{GC}$ chromatograms of samples analyzed routinely for targeted compounds such as PCBs can now be further sorted, and data further processed when SCCPs, OH-PCBs, PCDEs or other halogenated non-targeted compounds are found. Previously saved data can be qualitatively and 
quantitatively interpreted, and historical trends can be determined offering several advantages to conventional approaches.

These studies also emphasize the importance of expanding the list of target contaminants in routine analysis of environmental samples and the need for more studies to assess the continuous exposure of the organisms/environment to these compounds or mixtures of compounds. The recent advances and publications in the field of $\mathrm{GC} \times \mathrm{GC}$ have shown that the technique has gained popularity in the environmental field. 


\section{References}

[1] Z. Liu, J.B. Phillips, Comprehensive two-dimensional gas chromatography using an on-column thermal modulator interface, J. Chromatogr. Sci. 29 (1991) 227-231.

[2] A.M. Muscalu, T. Górecki, Comprehensive two-dimensional gas chromatography in environmental analysis, Trends Anal. Chem. 106 (2018) 225-245

[3] S. E. Prebihalo, K. L. Berrier, C. E. Freye, H. D. Bahaghighat, N. R. Moore, D. K. Pinkerton,

R. E. Synovec, Multidimensional gas chromatography: advances in instrumentation, chemometrics, and applications, Anal. Chem. (2018) 90, 505-532.

[4] M Adahchour, J. Beens, U.A.Th. Brinkman, Recent developments in the application of comprehensive two-dimensional gas chromatography, J. Chromatogr. A 1186 (2008) 67-108.

[5] P.Q. Tranchida, F.A. Franchina, P. Dugo, L. Mondello, Comprehensive two-dimensional gas chromatography-mass spectrometry: recent evolution and current trends, Mass Spectrom. Rev. 35 (2016) 524-534.

[6] M. Edwards, A. Mostafa, T. Górecki, Modulation in comprehensive two-dimensional gas chromatography: 20 years of innovation, Anal. Bioanal. Chem. 401 (2011) 2335.

[7] P. Q. Tranchida, Comprehensive two-dimensional gas chromatography: a perspective on processes of modulation, J. Chromatogr. A 1536 (2018) 2-5.

[8] L. Mondello, P.Q. Tranchida, P. Dugo, G. Dugo, Comprehensive two- dimensional gas chromatography-mass spectrometry: a review, Mass Spectrom. Rev. 27 (2008) 101-124.

[9] P. Q. Tranchida, Current state of comprehensive two-dimensional gas chromatography-mass spectrometry with focus on processes of ionization, Trends Anal. Chem. 105 (2018) 360-366.

[10] A. Mostafa, M. Edwards, T. Górecki, Optimization aspects of comprehensive twodimensional gas chromatography, J. Chromatogr. A, 1255 (2012) 38- 55.

[11] J. Dallüge, J. Beens, U.A. Brinkman, Comprehensive two-dimensional gas chromatography: a powerful and versatile analytical tool, J. Chromatogr. A 1000 (2003) 69-108. 
[12] D. Bahaghighat, E. Freye, E. Synovec, Recent advances in modulator technology for comprehensive two dimensional gas chromatography, Trends Anal. Chem. 113 (2019) 379-391.

[13] A. Mostafa, T. Gorecki, Development and design of a single-stage cryogenic modulator for comprehensive two-dimensional gas chromatography, Anal. Chem. 88 (2016) 5414-5423.

[14] J.F. Griffith, W.L. Winniford, K. Sun, Rob E.J.C. Luong, A reversed-flow differential flow modulator for comprehensive two-dimensional gas chromatography, J. Chromatogr. A 1226 (2012) 116- 123.

[15] R.L. Firor, Comprehensive flow modulated two-dimensional gas chromatography system, Agilent Application Brief 2014 (2007) 4.

[16] FLUX flow modulator. https://www.leco.com/flux-gcxgc-flow-modulator, retrieved on April 9,2019

[17] Stockholm Convention Secretariat (2001) UNEP. Available at http://chm.pops.int. Accessed on February 15, 2018.

[18] P. Lepom, B. Brown, G. Hanke, R. Loos, P. Quevauviller, J. Wollgast, Needs for reliable analytical methods for monitoring chemical pollutants in surface water under the European Water Framework Directive, J. Chromatogr. A 1216 (2009) 302-315.

[19] E. Engel, J. Ratel, P. Blinet, S. Chin, G. Rose, P.J. Marriott, Benchmarking of candidate detectors for multiresidue analysis of pesticides by comprehensive two-dimensional gas chromatography, J. Chromatogr. A 1311 (2013) 140-148.

[20] Directive 2000/60/EC of the European Parliament and of the Council of 23 October 2000 establishing a framework for Community action in the field of water policy. Available at https://www.eea.europa.eu/policy-documents/directive-2000-60-ec-of, accessed February 16, 2018.

[21] Canadian Environmental Protection Act, 1999. Available at http://lawslois.justice.gc.ca/eng/acts/c-15.31/. Accessed on February 16, 2018.

[22] M. Tobiszewski, P. Bigus, J. Namieśnik, Determination of parent and methylated polycyclic aromatic hydrocarbons in water samples by dispersive liquid-liquid microextraction - two 
dimensional gas chromatography - time-of-flight mass spectrometry, Anal. Methods. 6 (2014) 6678-6687.

[23] A.M. Sulej-Suchomska, Ż Polkowska, T. Chmiel, T.M. Dymerski, Z.J. Kokot, J Namieśnik, Solid phase microextraction-comprehensive two-dimensional gas chromatography-time-of-flight mass spectrometry: a new tool for determining PAHs in airport runoff water samples, Anal. Methods 8 (2016) 4509-4520.

[24] V.P. Beškoski, S. Miletić, M. Ilić, G. Gojgić-Cvijović, P. Papić, N. Marić, T. ŠolevićKnudsen, B.S. Jovančićević, T. Nakano, M.M. Vrvić, Biodegradation of isoprenoids, steranes, terpanes, and phenanthrenes during in situ bioremediation of petroleum-contaminated groundwater, Clean Soil Air Water, 45(2) (2017), n/a, 1600023.

[25] N. Ochiai, T. Ieda, K. Sasamoto, Y. Takazawa, S. Hashimoto, A. Fushimi, K. Tanabe, Stir bar sorptive extraction and comprehensive two-dimensional gas chromatography coupled to highresolution time-of-flight mass spectrometry for ultra-trace analysis of organochlorine pesticides in river water (Report), J. Chromatogr. A 1218 (2011) 6851-6860.

[26] G. Purcaro, P. Quinto Tranchida, L. Conte, A. Obiedzińska, P. Dugo, G. Dugo, L. Mondello, Performance evaluation of a rapid-scanning quadrupole mass spectrometer in the comprehensive two-dimensional gas chromatography analysis of pesticides in water, J. Sep. Sci. 34 (2011) 24112417.

[27] Ontario Ministry of the Environment and Climate Change. Method E3487 (2010).

[28] A.M. Muscalu, E.J. Reiner, S. Liss, T. Chen, G. Ladwig, D. Morse, A routine accredited method for the analysis of polychlorinated biphenyls, organochlorine pesticides, chlorobenzenes and screening of other halogenated organics in soil, sediment and sludge by GCxGC- $\mu$ ECD, Anal. Bioanal. Chem. 401 (2011) 2403-2413.

[29] E. Jover, V. Matamoros, J.M. Bayona, Characterization of benzothiazoles, benzotriazoles and benzosulfonamides in aqueous matrixes by solid-phase extraction followed by comprehensive two-dimensional gas chromatography coupled to time-of-flight mass spectrometry, J. Chromatogr. A 1216 (2009) 4013-4019. 
[30] V. Matamoros, E. Jover, J. Bayona, Part-per-trillion determination of pharmaceuticals, pesticides, and related organic contaminants in river water by solid-phase extraction followed by comprehensive two-dimensional gas chromatography time-of-flight mass spectrometry, Anal. Chem. 82 (2010) 699-706.

[31] S. Prebihalo, A. Brockman, J. Cochran, F.L. Dorman, Determination of emerging contaminants in wastewater utilizing comprehensive two-dimensional gas chromatography coupled with time-of-flight mass spectrometry, J. Chromatogr. A 1419 (2015) 109-115.

[32] R.P. Eganhouse, J. Pontolillo, R.B. Gaines, G.S. Frysinger, F.L.P. Gabriel, H-P.E. Kohler, W. Giger, L.B. Barber, Isomer-specific determination of 4-nonylphenols using comprehensive twodimensional gas chromatography/time-of-flight mass spectrometry, Environ.Sci.Technol. 43 (2009) 9306.

[33] C. Zhang, R.P. Eganhouse, J. Pontolillo, I.M. Cozzarelli, Y. Wang, Determination of nonylphenol isomers in landfill leachate and municipal wastewater using steam distillation extraction coupled with comprehensive two-dimensional gas chromatography/time-of-flight mass spectrometry, J. Chromatogr. A 1230 (2012) 110-116.

[34] C. Devos, F. David, P. Sandra, A new validated analytical method for the determination of tributyltin in water samples at the quantification level set by the European Union, J. Chromatogr. A 1261 (2012) 151-157.

[35] P.C.F. Lima Gomes, B.B. Barnes, Á.J. Santos-Neto, F.M. Lancas, N.H. Snow, Determination of steroids, caffeine and methylparaben in water using solid phase microextraction-comprehensive two dimensional gas chromatography-time of flight mass spectrometry, J. Chromatogr. A. 1299 (2013) 126-130.

[36] D Relić, A Popović, D Đorđević, J Čáslavský, Occurrence of synthetic musk compounds in surface, underground, waste and processed water samples in Belgrade, Serbia, Environ. Earth Sci. 76 (2017) 122.

[37] C. Li, D. Wang, N. Li, Q. Luo, X. Xu, Z. Wang, Identifying unknown by-products in drinking water using comprehensive two-dimensional gas chromatography-quadrupole mass spectrometry and in silico toxicity assessment, Chemosphere 163 (2016) 535-543. 
[38] M. Kopperi, J. Parshintsev, J. Ruiz-Jiménez, M.L. Riekkola, Nontargeted evaluation of the fate of steroids during wastewater treatment by comprehensive two-dimensional gas chromatography-time-of-flight mass spectrometry, Environ. Sci. Pollut. Res. Int. 23 (2016) 1700817017.

[39] Q. Guo, J. Yu, K. Yang, X. Wen, H. Zhang, Z. Yu, H. Li, D. Zhang, M. Yang, Identification of complex septic odorants in Huangpu River source water by combining the data from gas chromatography-olfactometry and comprehensive two-dimensional gas chromatography using retention indices, Sci. Total Environ. 556 (2016) 36-44.

[40] M. Gómez, S. Herrera, D. Solé, E. García-Calvo, A.R. Fernández-Alba, Automatic searching and evaluation of priority and emerging contaminants in wastewater and river water by stir bar sorptive extraction followed by comprehensive two-dimensional gas chromatography-time-offlight mass spectrometry, Anal. Chem. 83 (2011) 2638-2647.

[41] P. Bastos, P. Haglund, The use of comprehensive two- dimensional gas chromatography and structure-activity modeling for screening and preliminary risk assessment of organic contaminants in soil, sediment, and surface water, J Soils Sediments 12 (2012) 1079-1088.

[42] M. Wooding, E.R. Rohwer, Y. Naudé, Determination of endocrine disrupting chemicals and antiretroviral compounds in surface water: a disposable sorptive sampler with comprehensive gas chromatography - time-of-flight mass spectrometry and large volume injection with ultra-high performance liquid chromatography-tandem mass spectrometry, J. Chromatogr. A 1496 (2017) 122-132.

[43] K.M. Blum, P.L. Andersson, G. Renman, L. Ahrens, M. Gros, K. Wiberg, P. Haglund, Nontarget screening and prioritization of potentially persistent, bioaccumulating and toxic domestic wastewater contaminants and their removal in on-site and large-scale sewage treatment plants, Sci. Total Environ. 575 (2017) 265-275.

[44] E.M.M. Wanda, H. Nyoni, B.B. Mamba, T.A.M. Msagati, Occurrence of emerging micropollutants in water systems in Gauteng, Mpumalanga, and North West Provinces, South Africa, Int. J. Environ. Res. Public Health 14 (2017) 79. 
[45] M. Van Den Berg, L.S. Birnbaum, M. Denison, M. De Vito, W Farland, M. Feeley, H. Fiedler, H. Hakansson, A. Hanberg, L. Haws, M. Rose, S. Safe, D. Schrenk, C. Tohyama, A. Tritscher, J. Tuomisto, M. Tysklind, N. Walker, R.E. Peterson, The 2005 World Health Organization reevaluation of human and mammalian toxic equivalency factors for dioxins and dioxin-like compounds, Toxicol. Sci. 93 (2006) 223-241.

[46] M. Zapadlo, J. Krupčík, T. Kovalczuk, P. Májek, I. Špánik, D. Armstrong, P.Sandra, Enhanced comprehensive two-dimensional gas chromatographic resolution of polychlorinated biphenyls on a non-polar polysiloxane and an ionic liquid column series, J. Chromatogr. A 1218 (2011) 746-751.

[47] D. Megson, R. Kalin, P. Worsfold, C. Gauchotte-Lindsay, D. Patterson, M. Lohan, S. Comber, T.A. Brown, G. O'Sullivan, Fingerprinting polychlorinated biphenyls in environmental samples using comprehensive two-dimensional gas chromatography with time-of-flight mass spectrometry, J. Chromatogr. A 1318 (2013) 276-283.

[48] P. Korytár, P.E.G. Leonards, J. de Boer, U.A.T. Brinkman, Group separation of organohalogenated compounds by means of comprehensive two-dimensional gas chromatography, J. Chromatogr. A 1086 (2005) 29-44.

[49] L.R. Bordajandi, J.J. Ramos, J. Sanz, M.J. González, L. Ramos, Comprehensive twodimensional gas chromatography in the screening of persistent organohalogenated pollutants in environmental samples, J. Chromatogr. A 1186 (2008) 312-324.

[50] K. Kalachova, J. Pulkrabova, T. Cajka, L Drabova, J Hajslova, Implementation of comprehensive two-dimensional gas chromatography-time-of-flight mass spectrometry for the simultaneous determination of halogenated contaminants and polycyclic aromatic hydrocarbons in fish, Anal. Bioanal. Chem. 403 (2012) 2813-2824.

[51] A.M. Booth, A.G. Scarlett, C.A. Lewis, S.T. Belt, S.J. Rowland, Unresolved complex mixtures (UCMs) of aromatic hydrocarbons: branched alkyl indanes and branched alkyl tetralins are present in UCMs and accumulated by and toxic to, the mussel Mytilus edulis, Environ. Sci. Technol. 42 (2008) 8122-8126. 
[52] E. Hoh, S.J. Lehotay, K. Mastovska, H.L. Ngo, W. Vetter, K. Pangallo, C.M. Reddy, Capabilities of direct sample introduction-comprehensive two-dimensional gas chromatographytime-of-flight mass spectrometry to analyze organic chemicals of interest in fish oils, Environ. Sci. Technol. 43 (2009) 3240-3247.

[53] E. Hoh, S.J. Lehotay, K.C. Pangallo, K. Mastovska, H.L. Ngo, C.M. Reddy, W. Vetter, Simultaneous quantitation of multiple classes of organohalogen compounds in fish oils with direct sample introduction comprehensive two-dimensional gas chromatography and time-of-flight mass spectrometry, J. Agric. Food Chem. 57 (2009) 2653-2660.

[54] E. Hoh, N.G. Dodder, S.J. Lehotay, K.C. Pangallo, C.M. Reddy, K.A. Maruya, Nontargeted comprehensive two-dimensional gas chromatography/time-of-flight mass spectrometry method and software for inventorying persistent and bioaccumulative contaminants in marine environments, Environ. Sci. Technol. 46 (2012) 8001-8008.

[55] N.J. Shaul, N.G. Dodder, L.I. Aluwihare, S.A. Mackintosh, K.A. Maruya, S.J. Chivers, K. Danil, D.W. Weller, E. Hoh, Nontargeted biomonitoring of halogenated organic compounds in two ecotypes of bottlenose dolphins (Tursiops truncatus) from the Southern California Bight, Environ. Sci. Technol. 49 (2015) 1328-1338.

[56] M. Pena-Abaurrea, A. Covaci, L. Ramos, Comprehensive two-dimensional gas chromatography-time-of-flight mass spectrometry for the identification of organobrominated compounds in bluefin tuna, J. Chromatogr. A 1218 (2011) 6995-7002.

[57] C.J. Millow, S.A. Mackintosh, R.L. Lewison, N.G. Dodder, E. Hoh, Identifying bioaccumulative halogenated organic compounds using a nontargeted analytical approach: seabirds as sentinels, PLoS ONE. 10 (5) (2015), e0127205.

[58] P. López, M. Tienstra, A. Lommen, H.G.J. Mol, Validation of an automated screening method for persistent organic contaminants in fats and oils by GC $\times$ GC-ToFMS, Food Chem. 211 (2016) 645-653.

[59] M Pena-Abaurrea, L. Weijs, L. Ramos, N. Borghesi, S. Corsolini, H. Neels, R. Blust, A. Covaci, Anthropogenic and naturally-produced organobrominated compounds in bluefin tuna from the Mediterranean Sea, Chemosphere 76 (2009) 1477-1482. 
[60] D Xia, L Gao, M Zheng, S Wang, G Liu, Simultaneous analysis of polychlorinated biphenyls and polychlorinated naphthalenes by isotope dilution comprehensive two-dimensional gas chromatography high-resolution time-of-flight mass spectrometry, Anal. Chim. Acta. 937 (2016) 160-167.

[61] M. Pena-Abaurrea, F. Ye, J. Blasco, L. Ramos, Evaluation of comprehensive twodimensional gas chromatography-time-of-flight-mass spectrometry for the analysis of polycyclic aromatic hydrocarbons in sediments, J. Chromatogr. A 1256 (2012) 222.

[62] T. Ieda, N. Ochiai, T. Miyawaki, T. Ohura, Y. Horii, Environmental analysis of chlorinated and brominated polycyclic aromatic hydrocarbons by comprehensive two-dimensional gas chromatography coupled to high-resolution time-of-flight mass spectrometry, J. Chromatogr. A 1218 (2011) 3224-3232.

[63] S. Fernando, K.J. Jobst, V.Y. Taguchi, P.A. Helm, E.J. Reiner, B.E. McCarry, Identification of the halogenated compounds resulting from the 1997 Plastimet Inc. fire in Hamilton, Ontario, using comprehensive two- dimensional gas chromatography and (ultra)high resolution mass spectrometry, Environ. Sci. Technol. 48 (2014) 10656-10663.

[64] K. Jobst, L. Shen, E. Reiner, V. Taguchi, P. Helm, R. McCrindle, S. Backus, The use of mass defect plots for the identification of (novel) halogenated contaminants in the environment, Anal. Bioanal. Chem. 405 (2013) 3289-3297.

[65] M. Ubukata, K.J. Jobst, E.J. Reiner, S.E. Reichenbach, Q. Tao, J. Hang, Z. Wu, A. J. Dane, R.B. Cody, Non-targeted analysis of electronics waste by comprehensive two-dimensional gas chromatography combined with high-resolution mass spectrometry: using accurate mass information and mass defect analysis to explore the data, J. Chromatogr. A 1395 (2015) 152-159. [66] P.M. Antle, C.D. Zeigler, Y. Gankin, A. Robbat, New spectral deconvolution algorithms for the analysis of polycyclic aromatic hydrocarbons and sulfur heterocycles by comprehensive twodimensional gas chromatography-quadrupole mass spectrometry, Anal. Chem. 85 (2013) 1036910376.

[67] S. Hashimoto, T. Yoshikatsu, F. Akihiro, I. Hiroyasu, T. Kiyoshi, S. Yasuyuki, U. Masa-aki, K. Akihiko, T. Kazuo, O. Hideyuki, A. Katsunori, Quantification of polychlorinated dibenzo-p- 
dioxins and dibenzofurans by direct injection of sample extract into the comprehensive multidimensional gas chromatograph/high-resolution time-of-flight mass spectrometer, J. Chromatogr. A 1178 (2008) 187-198.

[68] S. Hashimoto, Y. Takazawa, A. Fushimi, K. Tanabe, Y. Shibata, T. Ieda, N. Ochiai, H. Kanda, T. Ohura, Q. Tao, S.E. Reichenbach, Global and selective detection of organohalogens in environmental samples by comprehensive two-dimensional gas chromatography-tandem mass spectrometry and high-resolution time-of-flight mass spectrometry, J. Chromatogr. A 1218 (2011) 3799-3810.

[69] S. Hashimoto, Y. Zushi, A. Fushimi, Y. Takazawa, K. Tanabe, Y. Shibata, Selective extraction of halogenated compounds from data measured by comprehensive multidimensional gas chromatography/high resolution time-of-flight mass spectrometry for non-target analysis of environmental and biological samples, J. Chromatogr. A 1282 (2013) 183-189.

[70] Y. Zushi, S. Hashimoto, A. Fushimi, Y. Takazawa, K. Tanabe, Y. Shibata, Rapid automatic identification and quantification of compounds in complex matrices using comprehensive twodimensional gas chromatography coupled to high resolution time-of-flight mass spectrometry with a peak sentinel tool, Anal. Chim. Acta. 778 (2013) 54-62.

[71] Y. Zushi, S. Hashimoto, M. Tamada, S. Masunaga, Y. Kanai, K. Tanabe, Retrospective analysis by data processing tools for comprehensive two-dimensional gas chromatography coupled to high resolution time-of-flight mass spectrometry: a challenge for matrix-rich sediment core sample from Tokyo Bay, J. Chromatogr. A 1338 (2014) 117-126.

[72] Y. Zushi, J. Gros, Q. Tao, S.E. Reichenbach, S. Hashimoto, J.S. Arey, Pixel-by-pixel correction of retention time shifts in chromatograms from comprehensive two- dimensional gas chromatography coupled to high resolution time-of-flight mass spectrometry, J. Chromatogr. A 1508 (2017) 121-129.

[73] J. de Vos, P. Gorst-Allman, E. Rohwer, Establishing an alternative method for the quantitative analysis of polychlorinated dibenzo-p-dioxins and polychlorinated dibenzofurans by comprehensive two dimensional gas chromatography-time-of-flight mass spectrometry for developing countries, J. Chromatogr. A 1218 (2011) 3282-3290. 
[74] J. de Vos, R. Dixon, G. Vermeulen, P. Gorst-Allman, J. Cochran, E. Rohwer, J.F. Focant, Comprehensive two-dimensional gas chromatography time of flight mass spectrometry (GC $\times \mathrm{GC}$ TOFMS) for environmental forensic investigations in developing countries, Chemosphere 82 (2011) 1230-1239.

[75] C. Rimayi, L. Chimuka, D. Odusanya, J. de Boer, J. Weiss, Distribution of 2,3,7,8-substituted polychlorinated dibenzo-p-dioxin and polychlorinated dibenzofurans in the Jukskei and Klip/Vaal catchment areas in South Africa, Chemosphere 145 (2016) 314-321.

[76] A.M. Muscalu, E.J. Reiner, S. Liss, T. Chen, Determination of polychlorinated biphenyls, organochlorine pesticides, chlorobenzenes in sludge and sediment samples by GC $\times \mathrm{GC}-\mu \mathrm{ECD}$, Int. J. Environ. Anal. Chem. 90 (2010) 1-13.

[77] A.M. Muscalu, M. Edwards, T. Górecki, E.J. Reiner, Evaluation of a single-stage consumable-free modulator for comprehensive two-dimensional gas chromatography: Analysis of polychlorinated biphenyls, organochlorine pesticides and chlorobenzenes, J. Chromatogr. A 1391 (2015) 93-101.

[78] J.M. Da Silva, C.A. Zini, E.B. Caramao, Evaluation of comprehensive two-dimensional gas chromatography with micro-electron capture detection for the analysis of seven pesticides in sediment samples, J. Chromatogr. A 1218 (2011) 3166-3172.

[79] P. Korytár, P.E.G. Leonards, J. de Boer, U.A.Th. Brinkman, High-resolution separation of polychlorinated biphenyls by comprehensive two-dimensional gas chromatography, J. Chromatogr. A 958 (2002) 203-218.

[80] M. Harju, C. Danielsson, P. Haglund, Comprehensive two-dimensonal gas chromatography of 209 polychlorinated biphenyls, J. Chromatogr. A 1019 (2003) 111-126.

[81] P. Korytár, P. Haglund, J. de Boer, U.A.Th. Brinkman, Comprehensive two-dimensional gas chromatography for the analysis of organohalogenated micro-contaminants, TrAC Trends Anal. Chem. 25 (2006) 373-396.

[82] Y. Naudé, E.R. Rohwer, Two multidimensional chromatographic methods for enantiomeric analysis of o, $\mathrm{p}^{\prime}$-DDT and o, $\mathrm{p}^{\prime}$-DDD in contaminated soil and air in a malaria area of South Africa, Anal. Chim. Acta 730 (2012) 120-126. 
[83] V. Fernandes, S. Lehotay, L. Geis-Asteggiante, H. Kwon, H.G.J. Mol, H. van Der Kamp, N. Mateus, V.F. Domingues, C. Delerue-Matos, Analysis of pesticide residues in strawberries and soils by GC-MS/MS, LC-MS/MS and two-dimensional GC-time-of-flight MS comparing organic and integrated pest management farming, Food Addit. Contam. Part A 31 (2014) 262-270.

[84] S. Zhu, L. Gao, M. Zheng, H. Liu, B. Zhang, L. Liu, Y. Wang, Determining indicator toxaphene congeners in soil using comprehensive two- dimensional gas chromatography-tandem mass spectrometry, Talanta 118 (2014) 210-216.

[85] X. Liu, D. Li, J. Li, G. Rose, P.J. Marriott, Organophosphorus pesticide and ester analysis by using comprehensive two-dimensional gas chromatography with flame photometric detection, J. Hazard. Mater. 263 (2013) 761-767.

[86] Q. Gu, F. David, F. Lynen, K. Rumpel, G. Xu, P. De Vos, P. Sandra, Analysis of bacterial fatty acids by flow modulated comprehensive two-dimensional gas chromatography with parallel flame ionization detector/mass spectrometry, J. Chromatogr. A 1217 (2010) 4448.

[87] Q. Gu, F David, F Lynen, P Vanormelingen, W Vyverman, K Rumpel, G. Xu, P.Sandra, Evaluation of ionic liquid stationary phases for one dimensional gas chromatography-mass spectrometry and comprehensive two dimensional gas chromatographic analyzes of fatty acids in marine biota, J. Chromatogr. A 1218 (2011) 3056-3063.

[88] A. Zeng, S. Chin, A. Patti, P. Marriott, Profiling of soil fatty acids using comprehensive twodimensional gas chromatography with mass spectrometry detection, J. Chromatogr. A 1317 (2013) $239-245$.

[89] R Costa, A. Albergamo, M. Piparo, G. Zaccone, G. Capillo, A. Manganaro, P. Dugo, L. Mondello, Multidimensional gas chromatographic techniques applied to the analysis of lipids from wild-caught and farmed marine species, Eur. J. Lipid Sci. Technol. 119 (2017) 1600043.

[90] M.R. Jacobs, M. Edwards, T. Górecki, P.N. Nesterenko, R.A. Shellie, Evaluation of a miniaturised single-stage thermal modulator for comprehensive two-dimensional gas chromatography of petroleum contaminated soils, J. Chromatogr. A 1463 (2016) 162-168.

[91] P. Korytar, J. Parera, P.E.G. Leonards, F.J. Santos, J. De Boer, U.A.Th. Brinkman, Characterization of polychlorinated n-alkanes using comprehensive two-dimensional gas 
chromatography-electron-capture negative ionisation time-of-flight mass spectrometry.(Report), J. Chromatogr. A 1086 (2005) 71-82.

[92] D. Xia, L. Gao, M. Zheng, Q. Tian, H. Huang, L. Qiao, A novel method for profiling and quantifying short- and medium-chain chlorinated paraffins in environmental samples using comprehensive two-dimensional gas chromatography-electron capture negative ionization highresolution time-of-flight mass spectrometry, Environ. Sci. Technol. 50 (2016) 7601-7609.

[93] D. Xia, L. Gao, S. Zhu, M. Zheng, Separation and screening of short-chain chlorinated paraffins in environmental samples using comprehensive two-dimensional gas chromatography with micro electron capture detection, Anal. Bioanal. Chem. 406 (2014) 7561-7570.

[94] A.M. Muscalu, D. Morse, E.J. Reiner, T. Górecki, The quantification of short-chain chlorinated paraffins in sediment samples using comprehensive two-dimensional gas chromatography with $\mu$ ECD detection, Anal. Bioanal. Chem. 409 (2016) 2065-2074.

[95] E Skoczyńska, P. Korytár, J de Boer, Maximizing chromatographic information from environmental extracts by GCxGC-ToF-MS, Environ. Sci. Technol. 42 (2008) 6611-6618.

[96] D.C. Hilton, R.S. Jones, A. Sjödin, A method for rapid, non-targeted screening for environmental contaminants in household dust, J. Chromatogr. A 1217 (2010) 6851-6856.

[97] M. Pena-Abaurrea, K.J. Jobst, R. Ruffolo, L. Shen, R. McCrindle, P.A. Helm, E.J. Reiner, Identification of potential novel bioaccumulative and persistent chemicals in sediments from Ontario (Canada) using scripting approaches with GC×GC-TOF MS analysis, Environ. Sci. Technol. 48 (2014) 9591.

[98] N. Zhan, F. Guo, Q. Tian, Z.P. Yang, Z. Rao, Screening and quantification of organic pollutants in soil using comprehensive two- dimensional gas chromatography with time-of-flight mass spectrometry, Anal. Lett. 51 (2018) 955-970.

[99] C. Veenaas, P. Haglund, Methodology for non-target screening of sewage sludge using comprehensive two-dimensional gas chromatography coupled to high-resolution mass spectrometry, Anal. Bioanal. Chem. 409 (2017) 4867-4883. 
[100] A.C. Lewis, N. Carslaw, P.J. Marriott, R.M. Kinghorn, P. Morrison, A.L. Lee, K.D. Bartle, M.J. Pilling, A larger pool of ozone-forming carbon compounds in urban atmospheres, Nature. 405 (2000) 778-781.

[101] O. Panić, T. Górecki, Comprehensive two-dimensional gas chromatography (GC×GC) in environmental analysis and monitoring, Anal. Bioanal. Chem. 386 (2006) 1013-1023.

[102] J.F. Hamilton, Using comprehensive two-dimensional gas chromatography to study the atmosphere, J. Chromatogr. Sci. 48 (2010) 274-282.

[103] C. Arsene, D. Vione, N. Grinberg, R.I. Olariu, GC $\times$ GC-MS hyphenated techniques for the analysis of volatile organic compounds in air, J. Liq. Chromatogr. Rel. Technol. 34 (2011) 10771111.

[104] N. Ochiai, T. Ieda, K. Sasamoto, A. Fushimi, S. Hasegawa, K. Tanabe, S. Kobayashi, Comprehensive two-dimensional gas chromatography coupled to high-resolution time-of-flight mass spectrometry and simultaneous nitrogen phosphorous and mass spectrometric detection for characterization of nanoparticles in roadside atmosphere, J. Chromatogr. A 1150 (2007) 13-20.

[105] A. Fushimi, K. Saitoh, Y. Fujitani, S. Hasegawa, K. Takahashi, K. Tanabe, S. Kobayashi, Organic-rich nanoparticles (diameter: $10-30 \mathrm{~nm}$ ) in diesel exhaust: fuel and oil contribution based on chemical composition, Atmos. Environ. 45 (2011) 6326-6336.

[106] A. Fushimi, S. Hashimoto, T. Ieda, N. Ochiai, Y. Takazawa, Y. Fujitani, K. Tanabe, Thermal desorption - comprehensive two-dimensional gas chromatography coupled with tandem mass spectrometry for determination of trace polycyclic aromatic hydrocarbons and their derivatives, J. Chromatogr. A 1252 (2012) 164-170.

[107] C. Manzano, E. Hoh, S.L.M. Simonich, Improved separation of complex polycyclic aromatic hydrocarbon mixtures using novel column combinations in GC×GC/ToF-MS, Environ. Sci. Technol. 46 (2012) 7677-7684.

[108] C. Manzano, E. Hoh, S.L.M. Simonich, Quantification of complex polycyclic aromatic hydrocarbon mixtures in standard reference materials using comprehensive two-dimensional gas chromatography with time-of-flight mass spectrometry, J. Chromatogr. A 1307 (2013) 172-179. 
[109] Y. Wang, X. Xu, L. Yin, H. Cheng, T. Mao, K. Zhang, W. Lin, Z. Meng, J.A. Palasota, Coupling of comprehensive two-dimensional gas chromatography with quadrupole mass spectrometry: application to the identification of atmospheric volatile organic compounds, J. Chromatogr. A 1361 (2014) 229-239.

[110] J. Lee, S.K. Sayler, M. Zhou, H. Zhu, R.J. Richardson, R. Neitzel, K. Kurabayashi, X. Fan, On-site monitoring of occupational exposure to volatile organic compounds by a portable comprehensive 2-dimensional gas chromatography device, Anal. Methods 10 (2018) 237-244.

[111] C. Lipok, J. Hippler, O.J. Schmitz, A four dimensional separation method based on continuous heart-cutting gas chromatography with ion mobility and high resolution mass spectrometry, J. Chromatogr. A 1536 (2018) 50-57.

[112] D. Muir, Chlorinated paraffins. In: The Handbook of Environmental Chemistry, vol. 3; Paasivirta J., Ed.; Springer-Verlag: Berlin, Heidelberg (2000). pp. 203-236.

[113] G.T. Tomy, Analysis of chlorinated paraffins in environmental matrices: the ultimate challenge for the analytical chemist. In: The Handbook of Environmental Chemistry, vol. 10. Boer J, editors Springer, Berlin, Heidelberg, (2010), pp. 83-106.

[114] K.G. Drouillard, G.T. Tomy, D.C.G. Mui, K.J. Friesen, Volatility of chlorinated n-alkanes (C10 - C12): vapor pressures and Henry's law constants, Environ. Toxicol. Chem. 17(7) (1998) 1252-1260.

[115] K.G. Drouillard, T. Hiebert, P. Tran, G.T. Tomy, D.C.G. Muir, K.J. Friesen, Estimating the aqueous solubilities of individual chlorinated $n$-alkanes $(\mathrm{C} 10-\mathrm{C} 12)$ from measurements of chlorinated alkane mixtures, Environ. Toxicol. Chem. 17(7) (1998) 1261-1267.

[116] Environment Canada 2008; Chlorinated paraffins. Accessed 14 Sep 2015. http://www.ec.gc.ca/lcpe-cepa/default.asp?lang=En\&n=D7D84872-1\&offset=11

[117] H. Fiedler, Short-chain chlorinated paraffins: production, use and international regulations. In: The Handbook of Environmental Chemistry, vol. 10. Boer J. editors; Springer-Verlag, Berlin, (2000) 1-40.

[118] Environment and Climate Change Canada, Priority Substances Assessment Program, Retrieved from https://ec.gc.ca/ese-ees/default.asp?lang=En\&n=3E5A065C-1 
[119] Z. Zencak, M. Oehme, Recent developments in the analysis of chlorinated paraffins, Trends Anal. Chem. 25(4) (2006) 310-317.

[120] L.M. Van Mourik, P.E. Leonards, C. Gaus, J. De Boer, Recent developments in capabilities for analysing chlorinated paraffins in environmental matrices: a review, Chemosphere. (2015) 136259.

[121] R. Tehrani, B. Van Aken, Hydroxylated polychlorinated biphenyls in the environment: sources, fate, and toxicities, Environ. Sci. Pollut. R.Environ. Sci. Pollut. R. 21(10) (2014) 1-12 [122] F. A. Grimm, D. Hu, I. Kania-Korwel, H. Lehmler., G. Ludewig, K.C. Hornbuckle, M.W. Duffel, A. Bergman, L.W. Robertson, Metabolism and metabolites of polychlorinated biphenyls, Crit. Rev. Toxicol. 45(3) (2015) 245-272.

[123] A. Buckman, C. S. Wong, E.A. Chow, S.B. Brown, K.R Solomon, A.T Fisk, Biotranformation of polychlorinated biphenyls (PCBs) and biotranformation of hydroxylated PCBs in fish, Aquat. Toxicol. 78 (2006) 176-185.

[124] I. Kania-Korwel, H. Lehmler, Chiral polychlorinated biphenyls: absorption, metabolism and excretion - a review, Environ. Sci. Pollut. R. 23(3) (2016) 2042-2057.

[125] D. Ueno, C. Darling, M. Alee, L. Campbell, G. Pacepavicius, C. Teixeira, D. Muir, Detection of hydroxylated polychlorinated biphenyls (OH-PCBs) in the abiotic environment: surface water and precipitation from Ontario, Canada, Environ. Sci. Technol. 41(6) (2007) 1841-1848.

[126] Muir D, Detection of hydroxylated polychlorinated biphenyls (OH-PCBs) in the abiotic environment: surface water and precipitation from Ontario, Canada, Environ. Sci. Technol. 41(6) (2007) 1841-1848.

[127] R.F. Marek, P.S. Thorne, K. Wang, J. DeWall, K.C. Hornbuckle, PCB and OH-PCB in serum from children and mothers in urban and rural communities, Environ. Sci. Technol. 47 (2013) 3353-3361.

[128] W.X. Koh, K.C. Hornbuckle, P.S. Thorne, Human serum from urban and rural adolescents and their mothers shows exposure to polychlorinated biphenyls not found in commercial mixtures, Environ. Sci. Technol., 49(13) (2015) 8105. 
[129] K. Nomiyama, Y. Uchiyama, S. Horiuchi, A. Eguchi, H. Mizukawa, S.H. Hirata, R. Shinohara, S. Tanabe, Organohalogen compounds and their metabolites in the blood of Japanese Amberjack (Seriola quinqueradiata) and Scalloped Hammerhead Shark (Sphyrna lewini) from Japanese coastal waters, Chemosphere 85 (2011) 315-321

[130] S. Tartu, S. Bourgeon, J. Aars, M. Andersen, A. Polder, G.W. Thiemann, J.M. Welker, H. Routti, Sea ice-associated decline in body condition leads to increased concentrations of lipophilic pollutants in polar bears (Ursus Maritimus) from Svalbard, Norway, Sci. Total Environ. 576 (2017) 409-419.

[131] R.F. Marek, A. Martinez, K.C. Hornbuckle, Discovery of hydroxylated polychlorinated biphenyls (OH-PCBs) in sediment from a Lake Michigan waterway and original commercial Aroclors, Environ. Sci. Technol. 47(15) (2013) 8204.

[132] J. Sun, L. Zhu, L. Pan, Y. Song, Y. Zhang, L. Qu, Y. Zhan, Detection of methoxylated and hydroxylated polychlorinated biphenyls in sewage sludge in China with evidence for their microbial transformation, Scientific Reports (2016) DOI: 10.1038, http://www.nature.com/articles/srep29782. Retrieved on June 22, 2018.

[133] A.M. Awad., A. Martinez., R.F. Marek, K.C. Hornbuckle, Occurrence and distribution of two hydroxylated polychlorinated biphenyl congeners in Chicago air, Environ. Sci. Technol. Letters 3(2) (2016) 47-51.

[134] R.F. Marek, P.S. Thorne, N.J. Herkert, A.M. Awad, K.C. Hornbuckle, Airborne PCBs and OH-PCBs inside and outside urban and rural U.S. schools, Environ. Sci. Technol. 51 (2017) $7853-7860$.

[135] A. Agüera, A.R. Fernández-Alba, L. Piedra, M. Mézcua, M. José Gómez, Evaluation of triclosan and biphenylol in marine sediments and urban wastewaters by pressurized liquid extraction and solid phase extraction followed by gas chromatography mass spectrometry and liquid chromatography mass spectrometry, Anal. Chim. Acta 480 (2003) 193-205.

[136] A. Eguchi, K. Nomiyama, M. Ochiai, H. Mizukawa, Y. Nagano, K. Nakagawa, K. Tanaka, H. Miyagawa, S. Tanabe, Simultaneous detection of multiple hydroxylated polychlorinated 
biphenyls from a complex tissue matrix using gas chromatography/isotope dilution mass spectrometry, Talanta 118 (2014) 253-261.

[137] M. Becker, T. Phillips, S. Safe, Polychlorinated diphenyl ethers - a review, Toxicol. Environ. Chem. 33 (1991) 189-200.

[138] J. Koistinen, Polychlorinated diphenyl ethers. In: Paasivirta J, editor. The Handbook of Environmental Chemistry, vol. 3. Berlin, Germany' Springer-Verlag; 2000. p. 157- 201. Part K.

[139] J.L. Domingo, Polychlorinated diphenyl ethers (PCDEs): environmental levels, toxicity and human exposure a review of the published literature, Environ. Int. 32 (2006) $121-127$.

[140] J. Koistinen, PCDEs: sources and environmental fate, Toxicol. Environ. Chem. 66:1-4 (1998) 27-35.

[141] J. Paasivirta, J. Tarhnen, J. Soikkeli, Occurrence and fate of polychlorinated diphenyl ethers (PCDE, PCA, PCV, PCPA and PCBA) in environment, Chemosphere Vol.15(9-12) (1986) 14291433.

[142] J-S Yang, S-L Lin, T-C Lin, Y-L Wu, L-C Wang, G-P Chang-Chien, Emissions of polychlorinated diphenyl ethers from a municipal solid waste incinerator during the start-up operation, J. Hazard. Mater. 299 (2015) 206-214.

[143] J. Paasivirta, J. Koistinen, Chlorinated ethers. In: Kiceniuk JW, Ray S (eds) Analysis of Contaminants in Edible Aquatic Resources (1994) Pan 4, pp 411-427.

[144] C.J. Stafford, Halogenated diphenyl ethers identified in avian tissues and eggs by GC/MS, Chemosphere 12(12) (1983) 1487-1495.

[145] A.J. Niimi, C.D. Metcalfe, S.Y. Huestis, Chlorinated diphenyl ethers in Great Lakes fish and their environmental implications, Environ. Toxicol. Chem. 13(7) (1994) 1133-1138.

[146] J. Koistinen, J. Koivusaari, I. Nuuja, J. Paasivirta, PCDEs, PCBs, PCDDs and PCDFs in black guillemots and white tailed eagles from the Baltic Sea, Chemosphere 30(9) (1995) 16711684. 
[147] S. Rayne, Development of a Multiple-class high-resolution gas chromatographic relative retention time model for halogenated environmental contaminants, Anal. Chem., 75(2003) 10491057.

[148] R. Weber, A. Watson, M. Forter, F. Oliaei, Persistent organic pollutants and landfills - a review of past experiences and future challenges, Waste. Manag. Res. 29 (2011) 107-121.

[149] E.J. Reiner, A.R. Boden, T. Chen, K. MacPherson, A.M. Muscalu, Advances in the analysis of persistent halogenated organic compounds, LCGC Europe. 2 (2010) 60-70.

[150] J.C. Giddings, Two-Dimensional separations: concept and promise, Anal. Chem. 56 (1984) 1256.

[151] J.C. Giddings, Concepts and comparisons in multidimensional separation, J. High. Res. Chromatogr. \& Chromatogr. Comm. 10 (1987) 319-323.

[152] LECO Corporation, Pegasus 4D GC×GC-TOFMS Brochure 2014 (2014) 8.

[153] S. Ly-Verdu, A. Schaefer, M. Kahle, T. Groeger, S. Neschen, J. Arteaga-Salas, M. Ueffing, M.H. Angelis, R. Zimmermann, The impact of blood on liver metabolite profiling - a combined metabolomic and proteomic approach, Biomed. Chromatogr. 28 (2014) 231-240.

[154] S.M. Rocha, M. Caldeira, J. Carrola, M. Santos, N. Cruz, I.F. Duarte, Exploring the human urine metabolomic potentialities by comprehensive two-dimensional gas chromatography coupled to time of flight mass spectrometry, J. Chromatogr. A 1252 (2012) 155-163.

[155] J. Jeong, X. Shi, X. Zhang, S. Kim, C. Shen, Model-based peak alignment of metabolomic profiling from comprehensive two-dimensional gas chromatography mass spectrometry, BMC Bioinf. (2012) 13-27.

[156] K.D. Nizio, J.J. Harynuk, Analysis of alkyl phosphates in petroleum samples by comprehensive two-dimensional gas chromatography with nitrogen phosphorus detection and post-column Deans switching, J. Chromatogr. A. 1252 (2012) 171-176.

[157] S. Li, J. Cao, S. Hu, D. Zhang, R. Fan, Analysis of terpanes in biodegraded oils from China using comprehensive two-dimensional gas chromatography with time-of-flight mass spectrometry, Fuel. 133 (2014) 153-162. 
[158] R.C. Silva, R.S.F. Silva, de Castro, V.R. Eustaquio, K.E. Peters, D.A. Azevedo, Extended diamondoid assessment in crude oil using comprehensive two-dimensional gas chromatography coupled to time-of-flight mass spectrometry, Fuel. 112 (2013) 125-133.

[159] L. Zhang, Z. Zeng, C. Zhao, H. Kong, X. Lu, G. Xu, A comparative study of volatile components in green, oolong and black teas by using comprehensive two-dimensional gas chromatography-time-of-flight mass spectrometry and multivariate data analysis, J. Chromatogr. A 1313 (2013) 245-252.

[160] J. Kiefl, P. Schieberle, Evaluation of process parameters governing the aroma generation in three hazelnut cultivars (Corylus avellana L.) by correlating quantitative key odorant profiling with sensory evaluation, J. Agric. Food Chem. 61 (2013) 5236-5244.

[161] J. Filippi, E. Belhassen, N. Baldovini, H. Brevard, U.J. Meierhenrich, Qualitative and quantitative analysis of vetiver essential oils by comprehensive two-dimensional gas chromatography and comprehensive two-dimensional gas chromatography/mass spectrometry, J. Chromatogr. A 1288 (2013) 127-148.

[162] D. Nabi, J. Gros, P. Dimitriou-Christidis, J.S. Arey, Mapping environmental partitioning properties of nonpolar complex mixtures by use of GC×GC, Environ. Sci. Technol. 48 (2014) 6814-6826.

[163] C.M. Rochman, C. Manzano, B.T. Hentschel, S.L.M. Simonich, E. Hoh, Polystyrene plastic: a source and sink for polycyclic aromatic hydrocarbons in the marine environment, Environ. Sci. Technol. 47 (2013) 13976-13984.

[164] D. Muir, Environmental levels and fate. In: The Handbook of Environmental Chemistry, vol. 10; Boer J. Ed.; Springer, Berlin, Heidelberg; 2010 pp. 107-133.

[165] G.S. Stern, G.T. Tomy, An overview of environmental levels and distribution of polychlorinated paraffins. Organohalogen Compd. 47 (2000)135-138.

[166] S. Geiß, M. Schneider, G. Donnevert, J.W. Einax, N. Lettmann, A. Rey, H. Lepper, B. Korner, T. Prey, B. Hilger, M. Engelke, M-P. Strub, H. Adrien, G. Sawal, D. Loffler, T. Schillings, I. Hussy, P. Steinbichl, S. Scharf, A Ruderisch, J.E. Olmos, F.J. Santos, A. Bartolome, J. Caixach, 
Validation interlaboratory trial for ISO 12010: water quality - determination of short-chain polychlorinated alkanes (SCCP) in water, Accred. Qual. Assur. 17(1) (2012) 15-25.

[167] S. Geiß, N. Lettman, A. Rey, H. Lepper, B. Korner, S. Mais S, T. Prey, B. Hilger, M. Engelke, S. Lebertz, C. Chatellier, G. Sawal, D. Loffler, T. Schillings, Preliminary interlaboratory trial for ISO/DIS 12010: determination of short chain polychlorinated alkanes (SCCP) in water, Clean-Soil Air Water 39(6) (2011) 537-42.

[168] C.R. Nicholls, C.R. Allchin, R.J. Law, Levels of short and medium chain length polychlorinated n- alkanes in environmental samples from selected industrial areas in England and Wales, Environ. Pollut. 114(3) (2001) 415.

[169] L. Zeng, H. Li, T. Wang, Y. Gao, K. Xiao, Y. Du, G. Jiang, Behavior, fate, and mass loading of short chain chlorinated paraffins in an advanced municipal sewage treatment plant, Environ. Sci. Technol. 47(2) (2013) 732.

[170] G.T. Tomy, G.A. Stern, W.L. Lockhart, D. Muir, Occurrence of C10-C13 polychlorinated n-alkanes in Canadian midlatitude and arctic lake sediments. Environ. Sci. Technol. 33(17) (1999) 2858-63.

[171] C.H. Marvin, S. Painte, G.T. Tomy, G.A. Stern, E. Braekevelt, D.C.G. Muir, Spatial and temporal trends in short-chain chlorinated paraffins in Lake Ontario sediments, Environ. Sci. Technol. 37(20) (2003) 4561.

[172] G.T. Tomy, D.C.G Muir, G.A. Stern, J.B. Westmore. Levels of C10-C13 polychloro-nalkanes in marine mammals from the Arctic and the St. Lawrence River Estuary, Environ. Sci. Technol. 34(9) (2000) 1615-1619.

[173] G.T. Tomy, G.A. Stern, D. Muir, A. Fisk, C.D. Cymbalisty, J. Westmore, Quantifying C10C13 polychloroalkanes in environmental samples by high-resolution gas chromatography electron capture negative ion high resolution mass spectrometry, Anal.Chem. 69(14) (1997) 2762-2771.

[174] L. Zeng, R. Chen, Z. Zhao, T. Wang, Y. Gao, A. Li, L. Sun, Spatial distributions and deposition chronology of short chain chlorinated paraffins in marine sediments across the Chinese Bohai and Yellow Seas, Environ. Sci. Technol. 47(20) (2013) 11449-11456. 
[175] M. Reth, Z. Zencak, M. Oehme, First study of congener group patterns and concentrations of short- and medium-chain chlorinated paraffins in fish from the North and Baltic Sea, Chemosphere. 58(7) (2005) 847-54.

[176] L. Saborido Basconcillo, S.M. Backus, D.J. McGoldrick, D. Zaruk, E. Sverko, D.C.G. Muir, Current status of short- and medium chain polychlorinated n-alkanes in top predatory fish across Canada, Chemosphere 127 (2015) 93-100.

[177] M. Nilsson, M. Waldebäck, G. Liljegren, H. Kylin, K.E. Markides, Pressurized-fluid extraction (PFE) of chlorinated paraffins from the biodegradable fraction of source-separated household waste, Fresenius J. Anal. Chem. 370(7) (2001) 913-918.

[178] M. Nilsson, S. Bengtsson, H. Kylin, Identification and determination of chlorinated paraffins using multivariate evaluation of gas chromatographic data, Environ. Pollut. 163(Complete) (2012) $142-148$.

[179] Stockholm Convention on Persistent Organic Pollutants http://chm.pops.int/Implementation/Alternatives/Overview/tabid/5834/Default.aspx

[180] E. Sverko, G.T. Tomy, C.H. Marvin, D.C.G Muir, Improving the quality of environmental measurements on short chain chlorinated paraffins to support global regulatory efforts, Environ. Sci. Technol. 46 (2012) 4697-4698.

[181] International Chlorinated Alkanes Industry Association; Newsletters http://www.eurochlor.org/chlorinated-alkanes-(casg)/international-cooperation.aspx

[182] P. Korytár, J. Parera, P.E.G. Leonards, F.J. Santos, J. de Boer, U.A.T. Brinkman, Characterization of polychlorinated n-alkanes using comprehensive two-dimensional gas chromatography-electron-capture negative ionisation time-of-flight mass spectrometry, J. Chromatogr. A 1086(1) (2005) 71-82.

[183] I. van der Veen, W. Cofino, S. Crum, J. De Boer, Interlaboratory study on the analysis of chlorinated paraffins in environmental matrices, phase 1. IVM Institute for Environmental Studies, Amsterdam, (2012) QUASIMEME Report W-12/11. 
[184] L. van Mourik, I. van der Veen, S. Crum, J. De Boer, Interlaboratory study on the analysis of chlorinated paraffins in environmental matrices, phase 3. IVM Institute for Environmental Studies, Amsterdam, (2014) QUASIMEME Report R-15/10.

[185] G.W. Johnson, J.F. Quensen III, J.F. Chiarenzelli, C.M. Coreen Hamilton. Polychlorinated biphenyls. In: R.D. Morrison, B.L. Murphy (Ed.), Environmental forensics: contaminant specific guide, Academic Press, Amsterdam, (2010) 187-226.

[186] L.A. Rodenburg, J. Guo, S. Du, G. Cavallo, Evidence for unique and ubiquitous environmental sources of 3,3'-dichlorobiphenyl (PCB 11), Environ. Sci. Technol. 44(8) (2009) 2816-2821, 10.1021/es901155h

[187] N.J. Herkert, J.C. Jahnke, K.C. Hornbuckle, Emissions of tetrachlorobiphenyls (PCBs 47, 51, and 68) from polymer resin on kitchen cabinets as a non-aroclor source to residential air. Environ. Sci. Technol. 52(9) (2018). 5154-5160.

[188] I. Kania-Korwel, W.M. Duffel, H.J Lehmler, Gas chromatographic analysis with chiral cyclodextrin phases reveals the enantioselective formation of hydroxylated polychlorinated biphenyls by rat liver microsomes, Environ. Sci. Technol. 45 (2011) 9590-9596.

[189] M.O. James, K.M. Kleino, Seasonal influences on PCB retention and biotransformation in fish, Environ. Sci. Pollut. R. International 21(10) (2014) 6324-33.

[190] J. Sun, L. Pan, L. Zhu, Formation of hydroxylated and methoxylated polychlorinated biphenyls by bacillus subtilis: new insights into microbial metabolism, Sci. Total Environ. 613614 (2018) 54-61.

[191] A.H. Buckman, S.B. Brown, P.F. Hoekstra, K.R. Solomon, A.T. Fisk, Toxicokinetics of three polychlorinated biphenyl technical mixtures in rainbow trout (Oncorhynchus mykiss), Environ. Toxicol. Chem. 23(7) (2004) 1725-1736.

[192] A.C. Dirtu, V.L.B. Jaspers, R. Cernat, H. Neels, A. Covaci, Distribution of PCBs, their hydroxylated metabolites, and other phenolic contaminants in human serum from two European countries, Environ. Sci. Technol. 44 (2010) 2876-2883. 
[193] B. Gomara, M. Athanasiadou, J.E. Quintanilla-Lopez, M.J. Gonzalez, A. Bergman, Polychlorinated biphenyls and their hydroxylated metabolites in placenta from Madrid mothers, Environ Sci Pollut Res. 19 (2012) 139-147.

[194] N. Quinete, T. Schettgen, J. Bertram, T. Kraus, Occurrence and distribution of PCB metabolites in blood and their potential health effects in humans: a review. Environ. Sci. Pollut. R. 21(20) (2014) 11951-11972.

[195] W.X. Koh, K.C. Hornbuckle, R.F. Marek, K. Wang, P.S. Thorne, Hydroxylated polychlorinated biphenyls in human sera from adolescents and their mothers living in two U.S. Midwestern communities, Chemosphere 147 (2016) 389-395.

[196] W.X. Koh, K.C. Hornbuckle, K. Wan, P.S. Thorne, Serum polychlorinated biphenyls and their hydroxylated metabolites are associated with demographic and behavioral factors in children and mothers, Environ. Int. 94(Complete) (2016) 538-545.

[197] L.M. Campbell, D.C.G. Muir, D.M. Whittle, S. Backus, R.J. Norstrom, A.T. Fisk, Hydroxylated PCBs and other chlorinated phenolic compounds in lake trout (Salvelinus namaycush) blood plasma from the Great Lakes region, Environ. Sci. Technol. 37 (2003) 17201725.

[198] E.A.M. Gilroy, D.C.G. Muir, M.E. McMaster, C. Darling, L.M. Campbell, S.R. deSolla, J.L. Parrot, S.B. Brown, J.P. Sherry, Polychlorinated biphenyls and their hydroxylated metabolites in wild fish from Wheatley Harbour area of concern, Ontario, Canada, Environ. Toxicol. Chem. 31 (12) (2012). 2788-2797.

[199] K.M. Gabrielsen, G.D. Villanger, E. Lie, M. Karimi, C. Lydersen, K.M. Kovacs, B.M. Jenssen, Levels and patterns of hydroxylated polychlorinated biphenyls (OH-PCBs) and their associations with thyroid hormones in hooded seal (Cystophora cristata) mother-pup pairs, Aquat. Toxicol. 105 (2011) 482- 491.

[200] J. Bytingsvik, E. Lie, J. Aars, A.E. Derocher, O. Wiig, B.M. Jenssen, PCBs and OH- PCBs in polar bear mother-cub pairs: a comparative study based on plasma levels in 1998 and 2008 (report), Sci. Total Environ. 417418 (2012) 117. 
[201] M. Ochiai, K. Nomiyama, T. Isobe, H. Mizukawa, T.K. Yamada, Y. Tajima, T. Matsuishi, M. Amano, S. Tanabe, Accumulation of hydroxylated polychlorinated biphenyls (OH-PCBs) and implications for PCBs metabolic capacities in three porpoise species, Chemosphere 92 (2013) 803-810.

[202] K. Nomiyama, S. Hirakawa, A. Eguchi, C. Kanbara, D. Imaeda, J. Yoo, T. Kunisue, Kim, H. Iwata, S. Tanabe, Toxicological assessment of polychlorinated biphenyls and their metabolites in the liver of baikal seal (pusa sibirica), Environ. Sci. Technol. 48(22) (2014) 13530-13539.

[203] L. Weij, S.D. Shaw, M.L. Berger, H. Neels, R. Blust, A. Covaci, Methoxylated PBDEs (MeO-PBDEs), hydroxylated PBDEs (HO-PBDEs) and hydroxylated PCBs (HO- PCBs) in the liver of harbor seals from the northwest Atlantic, Sci. Total Environ. 493 (2014) 606-614.

[204] C. Louis, A. Covaci, M. Stas, D.E. Crocker, G. Malarvannan, A.C. Dirtu, C. Debier, Bioaccumulation of hydroxylated polychlorinated biphenyls and pentachlorophenol in the serum of northern elephant seal pups (mirounga angustirostris), Environ. Res. 136(Complete) (2015) 441-448.

[205] H. Routti, M.S. Andersen, E. Fuglei, A. Polder, N.G. Yocco, Concentrations and patterns of hydroxylated polybrominated diphenyl ethers and polychlorinated biphenyls in arctic foxes (vulpes lagopus) from svalbard, Environ. Pollut. 216 (2016) 264-272.

[206] M.W. van den Dungen, D.E. Kok, A. Polder, Hoogenboom Ron L A P, S.P.J. van Leeuwen, W.T. Steegenga, A.J. Murk, Accumulation of persistent organic pollutants in consumers of eel from polluted rivers compared to marketable eel, Environ. Pollut. 219(Complete) (2016) 80-88. [207] È.A.M. Gilroy, D.C.G. Muir, M.E. McMaster, C. Darling, L.M. Campbell, M. Alaee, J.P. Sherry, Halogenated phenolic compounds in wild fish from Canadian areas of concern, Environ. Toxicol. Chem. 36(9) (2017) 2266-2273.

[208] C. Li, J. Liu, N. Wu, X. Pan, J. Feng, G. Al-Basher, A.A. Allam, R. Qu, Z. Wang, Photochemical formation of hydroxylated polychlorinated biphenyls (OH-PCBs) from decachlorobiphenyl (PCB-209) on solids/air interface, J. Haz. Mat. 378 (2019) 120758. 
[209] R. Kamata, F. Shiraishi, D. Nakajama, H. Takigami, H. Shiraishi, Mono-hydroxylated polychlorinated biphenyls are potent aryl hydrocarbon receptor ligands in recombinant yeast cells, Toxicology in Vitro 23 (2009) 736-743.

[210] A. Dreiem, S. Rykken, H.J. Lehmler, L.W. Robertson, F. Fonnum, Hydroxylated polychlorinated biphenyls increase reactive oxygen species formation and induce cell death in cultured cerebellar granule cells, Toxicol Appl Pharmacol 240 (2009) 306-313.

[211] R. Bhalla, R. Tehrani, B. Aken, Toxicity of hydroxylated polychlorinated biphenyls (HOPCBs) using the bioluminescent assay Microtox ${ }^{\circledR}$, Ecotoxicology 25(7) (2016) 1438-1444.

[212] S. Subramanian, J. Schnoor, B. Van Aken, Effects of polychlorinated biphenyls (PCBs) and their hydroxylated metabolite (OH-PCBs) on Arabidopsis Thaliana, Environ. Sci. Technol. 51(12) (2017) 7263.

[213] R.J. Letcher, E. Klasson-Wehler, A. Bergman, Methyl sulfone and hydroxylated metabolites of polychlorinated biphenyls. In: New types of persistent halogenated compounds; Paasivirta, J., Ed.; Springer-Verlag: Berlin-Heidelberg, 2000; Vol. 3, pp 315-359.

[214] M. Montano, A.C. Gutelb, A.T.J. Murk, Persistent Toxic burdens of halogenated phenolic compounds in humans and wildlife, Environ. Sci. Technol. 47 (2013) 6071-6081.

[215] S. Berghuis, A. Bos, P. Sauer, E. Roze, Developmental neurotoxicity of persistent organic pollutants: an update on childhood outcome, Arch. Toxicol. 89(5) (2015) 687- 709.

[216] S. Mizukami-Murata, K. Fujita, T. Nakano, Effect of lower chlorinated hydroxylatedpolychlorobiphenyls on development of PC12 cells, Environ. Sci. Pollut. R. 25 (2018) 1643416445.

[217] J. Zheng, C. He, S. Chen, X. Yan, M. Guo, M. Wang, Y.J. Yu Y.J, Yang, B. Mai, Disruption of thyroid hormone (TH) levels and TH-regulated gene expression by polybrominated diphenyl ethers (PBDEs), polychlorinated biphenyls (PCBs), and hydroxylated PCBs in e-waste recycling workers, Environ. Int. 102 (2017) 138-144.

[218] M. Machala, L. Blaha, H.J. Lehmler, M. Pliskova, Z. Majkova, P. Kapplova, I. Sovadinova, J. Vondracek, T. Malmberg, L.W. Robertson, Toxicity of hydroxylated and quinoid PCB metabolites: inhibition of gap junctional intercellular communication and activation of aryl 
hydrocarbon and estrogen receptors in hepatic and mammary cells, Chem. Res. Toxicol. 17(3) (2004) 340-347.

[219] K. Dhakal, S.G. Gadupudi, H.J. Lehmler, G. Ludewig, W.M. Duffel, W.L. Robertson. Sources and toxicities of phenolic polychlorinated biphenyls (OH-PCBs), Environ. Sci. Pollut. R. International 25 (2018) 16277-16290.

[220] K. Pěnčíková, L. Svrzkova, S. Strapakova, J. Neca, I. Bartonkova, Z. Dvorak, M. Hyzdalova, L.P. Pivnicka, H.J. Lehmler, X. Li, J. Vondracek, M. Machala, In-vitro profiling of toxic effects of prominent environmental lower-chlorinated PCB congeners linked with endocrine disruption and tumor promotion, Environ. Pollut. 237 (2018) 473-486.

[221] M. Kawano, J. Hasegawa, T. Enomoto, H. Onishi, Y. Nishio, M. Matsuda, T. Wakimoto, Hydroxylated polychlorinated biphenyls (OH-PCBs): recent advances in wildlife contamination study, Environ. Sci. (Tokyo, Jpn.) 12 (6) (2005) 315-324.

[222] L. Gustavson, T.M. Ciesielski, J. Bytingsvik, B. Styrishave, M. Hansen, E. Lie, J. Aars, B.M. Jenssen, Hydroxylated polychlorinated biphenyls decrease circulating steroids in female polar bears (ursus maritimus), Environ. Res. 138 (2015) 191-201.

[223] Method E3488, The determination of polychlorinated biphenyl congeners (PCBc), organochlorine pesticides (OC) and chlorobenzenes $(\mathrm{CB})$ in water by two-dimensional gas chromatography micro-electron capture detection (GCxGC- $\mu \mathrm{ECD})$, Ministry of the Environment, Conservation and Parks, Toronto, Ontario, Canada.

[224] G.M. Frame, J.W. Cochran, S.S. Bowad, Complete PCB congener distributions for 17 aroclor mixtures determined by 3 HRGC systems optimized for comprehensive, quantitative, congener-specific analysis, J. High Resol. Chromatogr 19 (1996) 657-668.

[225] D.L. Bedard, J.F. Quensen III, Microbial Reductive Dechlorination of polychlorinated biphenyls. In: Microbial transformation and degradation of toxic organic chemicals, L. Y. Young and C.E. Cerniglia, Eds. (1995) 127-216, Wiley-Liss, Inc., New York. 


\section{Appendix A}

\section{Comprehensive Two-Dimensional Gas Chromatography in Environmental Analysis}

Recent applications of GC×GC: Tables A.1 to A.4

Table A.1. - Recent applications of GCXGC for the analysis of aqueous samples

\begin{tabular}{|c|c|c|c|c|c|}
\hline Application & Target analytes & Sample Preparation & Instrumentation & $\begin{array}{l}\text { Comments / Method } \\
\text { Performance }\end{array}$ & Reference \\
\hline $\begin{array}{l}\text { Water: } \\
\text { Baltic Sea, local } \\
\text { rivers and lakes, } \\
\text { drinking water }\end{array}$ & $\begin{array}{l}29 \text { compounds: } \\
\text { parent PAHs, oxy-PAHs, nitro- } \\
\text { PAHs, methyl-PAHs }\end{array}$ & Extraction: DLLME & $\begin{array}{l}\text { GC } \times \text { GC-TOFMS } \\
{ }^{1} \text { D: ZB-5 } 30 \mathrm{~m} \times 0.25 \mathrm{~mm} \times 0.25 \mu \mathrm{m} \\
{ }^{2} \mathrm{D}: \mathrm{ZB}-501.6 \mathrm{~m} \times 0.1 \mathrm{~mm} \times 0.25 \mu \mathrm{m} \\
\text { Modulator: cryogenic using liquid nitrogen } \\
\text { Modulation: } 6 \mathrm{~s} \text {, hot pulse } 1.2 \mathrm{~s} \text {, cold pulse } 1.8 \mathrm{~s} \\
\text { Total run time: } 30 \mathrm{~min}\end{array}$ & $\begin{array}{l}\text { LODs: }<30 \mathrm{ng} / \mathrm{L} \text { for the lighter } \\
\text { PAHs and } 0.9 \mathrm{ng} / \mathrm{L} \\
\text { (naphthalene) }-500 \mathrm{ng} / \mathrm{L}(\mathrm{PAHs} \\
>278 \mathrm{u})\end{array}$ & {$[22]$} \\
\hline Storm water & PAHs: 16 compounds & & $\begin{array}{l}\text { GC } \times \text { GC-TOF-MS } \\
{ }^{1} \text { D: ZB-5MS } 30 \mathrm{~m} \text { x } 0.25 \mathrm{~mm} \times 0.25 \mu \mathrm{m} \\
{ }^{2} \mathrm{D}: \text { BPX-50 } 2 \mathrm{~m} \text { x } 0.1 \mathrm{~mm} \times 0.1 \mu \mathrm{m} \\
\text { Modulation : } 5 \mathrm{~s}\end{array}$ & $\begin{array}{l}\text { Quantitative: } \\
\text { MDL } 0.22 \text { to } 2.2 \mathrm{ng} / \mathrm{L} \\
\text { MQL } 0.67 \text { to } 6.5 \mathrm{ng} / \mathrm{L}\end{array}$ & {$[23]$} \\
\hline Groundwater & PAH, hydrocarbons & & $\begin{array}{l}\text { GC } \times \text { GC-MS } \\
{ }^{1} \mathrm{D}: \text { Rtx-5SilMS } 30 \mathrm{~m} \text { x } 0.25 \mathrm{~mm} \text { x } 0.25 \mu \mathrm{m}\end{array}$ & Qualitative data & [24] \\
\hline
\end{tabular}




\begin{tabular}{|c|c|c|c|c|c|}
\hline Application & Target analytes & Sample Preparation & Instrumentation & $\begin{array}{l}\text { Comments / Method } \\
\text { Performance }\end{array}$ & Reference \\
\hline & & & ${ }^{2} \mathrm{D}:$ Rxi-17Sil MS $1 \mathrm{~m}$ x $0.25 \mathrm{~mm}$ x $0.25 \mu \mathrm{m}$ & & \\
\hline River water & 23 OCP & $\begin{array}{l}\text { SBSE - thermal } \\
\text { desorption }\end{array}$ & $\begin{array}{l}\text { GC } \times \text { GC-HR-TOF-MS } \\
\text { 'D: DB5-MS } 10 \mathrm{~m} \text { x } 0.18 \mathrm{~mm} \text { x } 0.18 \mu \mathrm{m} \\
{ }^{2} \mathrm{D}: \mathrm{BPX}-501 \mathrm{~m} \text { x } 0.1 \mathrm{~mm} \times 0.1 \mu \mathrm{m} \\
\text { Modulation: } 4 \mathrm{~s}\end{array}$ & & [25] \\
\hline Drinking Water & 28 Pesticides & SPME & $\begin{array}{l}\text { GC } \times \text { GC-qMS } \\
\text { 'D: SLB-MS } 30 \mathrm{~m} \text { x } 0.25 \mathrm{~mm} \times 1.0 \mu \mathrm{m} \\
1 \mathrm{~m} \times 0.25 \mathrm{~mm} \text { uncoated column (double loop) } \\
{ }^{2} \mathrm{D}: \text { SLB-IL59 } 1 \mathrm{~m} \text { x } 0.1 \mathrm{~mm} \times 0.08 \mu \mathrm{m} \\
\text { Modulation: } 6 \mathrm{~s} \\
\text { MS Parameters: scan speed } 20000 \mathrm{amu} / \mathrm{s} \text {, mass range } \\
\mathrm{m} / \mathrm{z} 50-450 \\
\text { Splitless injection } \\
\text { Software: ChromSquare }\end{array}$ & LOQ: $0.03-0.084 \mathrm{mg} / \mathrm{L}$ & [26] \\
\hline $\begin{array}{l}\text { Water: } \\
\text { wastewater, } \\
\text { river water, raw } \\
\text { Sewage }\end{array}$ & $\begin{array}{l}12 \text { compounds: } \\
\text { benzothiazoles, benzotriazoles, } \\
\text { benzosulfonamides }\end{array}$ & Extraction: SPE & $\begin{array}{l}\text { GC } \times \text { GC-TOFMS } \\
\text { 'D: TRB5-MS } 30 \mathrm{~m} \times 0.25 \mathrm{~mm} \times 0.25 \mu \mathrm{m} \\
{ }^{2} \mathrm{D} \text { : TRB50-HT } 1 \text { or } 2 \mathrm{~m} \times 0.1 \mathrm{~mm} \times 0.1 \mu \mathrm{m} \\
\text { Other columns tested: } \\
{ }^{1} \mathrm{D} \text { : TRB1701-MS } 30 \mathrm{~m} \times 0.25 \mathrm{~mm} \times 0.25 \mu \mathrm{m}\end{array}$ & $\begin{array}{l}\text { LODs: } \\
5-56 \mathrm{ng} / \mathrm{L} \text { in river water } \\
10-112 \mathrm{ng} / \mathrm{L} \text { in wastewater }\end{array}$ & [29] \\
\hline
\end{tabular}




\begin{tabular}{|c|c|c|c|c|c|}
\hline Application & Target analytes & Sample Preparation & Instrumentation & $\begin{array}{l}\text { Comments / Method } \\
\text { Performance }\end{array}$ & Reference \\
\hline & & & $\begin{array}{l}{ }^{1} \mathrm{D}: \text { ZB-WAX } 20 \mathrm{~m} \times 0.25 \mathrm{~mm} \times 0.25 \mu \mathrm{m} \\
{ }^{2} \mathrm{D}: \text { TRB-5 } 1 \text { or } 2 \mathrm{~m} \times 0.1 \mathrm{~mm} \times 0.1 \mu \mathrm{m}\end{array}$ & & \\
\hline $\begin{array}{l}\text { River Water } \\
\text { Pharmaceuticals } \\
\text { and Personal } \\
\text { Care Products }\end{array}$ & $\begin{array}{l}97 \text { target compounds: } \\
13 \text { pharmaceuticals, } 18 \\
\text { plasticizers, } 8 \text { personal care } \\
\text { products, } 9 \text { acid herbicides, } 8 \\
\text { triazines, } 10 \text { organophosphorous } \\
\text { compounds, } 5 \text { phenylureas, } 12 \\
\text { organochlorine biocides, } 9 \\
\text { PAHs, } 5 \text { benzothiazoles and } \\
\text { benzotriazoles }\end{array}$ & SPE, derivatization & $\begin{array}{l}\text { GC } \times \text { GC-TOFMS } \\
{ }^{1} \mathrm{D} \text { : TRB5-MS } 30 \mathrm{~m} \times 0.25 \mathrm{~mm} \times 0.25 \mu \mathrm{m} \\
{ }^{2} \mathrm{D} \text { : TRB50-HT } 12 \mathrm{~m} \times 0.1 \mathrm{~mm} \times 0.1 \mu \mathrm{m} \\
\text { Other columns tested: } \\
{ }^{1} \mathrm{D} \text { : TRB1701-MS } 30 \mathrm{~m} \times 0.25 \mathrm{~mm} \times 0.25 \mu \mathrm{m} \\
{ }^{2} \mathrm{D} \text { : TRB5-MS } 2 \mathrm{~m} \times 0.1 \mathrm{~mm} \times 0.1 \mu \mathrm{m} \\
\text { Modulation: } 5 \mathrm{~s}\end{array}$ & $\begin{array}{l}\text { \% RSD: } 1 \% \text { to } 20 \% \\
\text { LODs: } 0.5 \text { to } 110 \mathrm{ng} / \mathrm{L} \\
\text { LOQs: } 2 \text { to } 185 \mathrm{ng} / \mathrm{L}\end{array}$ & {$[30]$} \\
\hline $\begin{array}{l}\text { Wastewater, } \\
\text { soil }\end{array}$ & $\begin{array}{l}\text { Pesticides and organohalogens, } \\
\text { halogenated benzotriazoles }\end{array}$ & $\begin{array}{l}\text { Water: LLE } \\
\text { Soil: ultrasonic } \\
\text { extraction }\end{array}$ & $\begin{array}{l}\text { GC } \times \text { GC-TOFMS } \\
{ }^{1} \text { D: Rxi-5 Sil MS 60m x } 0.25 \mathrm{~mm} \times 0.32 \mu \mathrm{m} \\
{ }^{2} \text { D: Rtx-200 } 1.3 \mathrm{~m} \text { x } 0.25 \mathrm{~mm} \text { x } 0.25 \mu \mathrm{m} \\
\text { Modulation: } 2.5 \mathrm{sec}\end{array}$ & $\begin{array}{l}\text { Targeted-discovery method: } \\
\text { GC×GC-TOFMS combined with } \\
\text { the reference method } \\
\text { (ChromaTOF software) }\end{array}$ & {$[31]$} \\
\hline $\begin{array}{l}\text { Water: } \\
\text { wastewater, } \\
\text { groundwater }\end{array}$ & Nonylphenol isomers & & $\begin{array}{l}\text { GC } \times \text { GC-TOFMS } \\
{ }^{1} \mathrm{D}: \text { DB5-MS } 30 \mathrm{~m} \times 0.25 \mathrm{~mm} \times 1.0 \mu \mathrm{m} \\
{ }^{2} \mathrm{D} \text { : Supelcowax } 102 \mathrm{~m} \times 0.1 \mathrm{~mm} \times 0.1 \mu \mathrm{m} \\
\text { Split injection }\end{array}$ & $\begin{array}{l}\text { Reproducibility ( \% RSD) } \leq 15.3 \\
\%\end{array}$ & {$[32]$} \\
\hline $\begin{array}{l}\text { Landfill } \\
\text { leachate, }\end{array}$ & Nonylphenol isomers & $\begin{array}{l}\text { Steam distillation } \\
\text { extraction }\end{array}$ & $\begin{array}{l}\text { GC } \times \text { GC-TOFMS } \\
{ }^{1} \mathrm{D}: \text { DB5-MS } 30 \mathrm{~m} \times 0.25 \mathrm{~mm} \times 1.0 \mu \mathrm{m}\end{array}$ & MDL: 0.16-15.06 ng/L & {$[33]$} \\
\hline
\end{tabular}




\begin{tabular}{|c|c|c|c|c|c|}
\hline Application & Target analytes & Sample Preparation & Instrumentation & $\begin{array}{l}\text { Comments / Method } \\
\text { Performance }\end{array}$ & Reference \\
\hline $\begin{array}{l}\text { municipal } \\
\text { wastewater }\end{array}$ & & & $\begin{array}{l}{ }^{2} \mathrm{D}: \text { Supelcowax } 102 \mathrm{~m} \times 0.1 \mathrm{~mm} \text { x } 0.1 \mu \mathrm{m} \\
\text { Split injection }\end{array}$ & & \\
\hline Surface water & Tributyltin (TBT) & $\begin{array}{l}\text { SBSE combined with } \\
\text { in-situ derivatization }\end{array}$ & $\begin{array}{l}\text { GC-GC-MS/MS } \\
{ }^{1} \mathrm{D}: \mathrm{HP}-5 \mathrm{MS} 30 \mathrm{~m} \times 0.25 \mathrm{~mm} \times 0.25 \mu \mathrm{m} \\
\text { Deans switch - heart-cut windows } \\
{ }^{2} \mathrm{D}: \mathrm{DB}-17 \mathrm{MS} 30 \mathrm{~m} \times 0.25 \mathrm{~mm} \times 0.25 \mu \mathrm{m}\end{array}$ & & {$[34]$} \\
\hline Water & $\begin{array}{l}\text { steroids, caffeine and } \\
\text { methylparaben }\end{array}$ & & $\begin{array}{l}\text { GC } \times \text { GC-TOF-MS } \\
{ }^{1} \text { D: Rtx-5 } 15 \mathrm{~m} \times 0.25 \mathrm{~mm} \times 0.25 \mu \mathrm{m} \\
{ }^{2} \text { D: Rxi-17 } 1.5 \mathrm{~m} \times 0.25 \mathrm{~mm} \times 0.25 \mu \mathrm{m} \\
\text { Modulation : } 5 \mathrm{~s}\end{array}$ & $\begin{array}{l}\text { LODs and LOQs from } 0.02 \text { to } \\
100 \mu \mathrm{g} / \mathrm{L} \\
\text { Linearity: } 0.6-1200 \mu \mathrm{g} / \mathrm{L}\end{array}$ & {$[35]$} \\
\hline $\begin{array}{l}\text { Waste and } \\
\text { processed water }\end{array}$ & 14 musks & & $\begin{array}{l}\text { GC } \times \text { GC-HR-TOF-MS } \\
{ }^{1} \text { D: SLB-5MS } 30 \mathrm{~m} \times 0.25 \mathrm{~mm} \times 0.25 \mu \mathrm{m} \\
{ }^{2} \mathrm{D}: \text { BPX-50 } 1.29 \mathrm{~m} \times 0.1 \mathrm{~mm} \times 0.1 \mu \mathrm{m} \\
\text { Modulation: } 4 \mathrm{~s}\end{array}$ & $\begin{array}{l}\text { Quantitative: } \\
\text { LODs: } 0.05 \text { to } 2.95 \mathrm{ng} / \mathrm{L} \text { LOQs: } \\
0.1 \text { to } 9.84 \mathrm{ng} / \mathrm{L}\end{array}$ & [36] \\
\hline Drinking water & $\begin{array}{l}\text { Disinfection by-products: } 170 \\
\text { DBPs identified } \\
58 \text { toxic or potentially toxic } \\
\text { DBPs }\end{array}$ & SPE & $\begin{array}{l}\text { GC } \times \text { GC-qMS } \\
{ }^{1} \mathrm{D} \text { : DB-5MS } 30 \mathrm{~m} \times 0.25 \mathrm{~mm} \times 0.25 \mu \mathrm{m} \\
{ }^{2} \mathrm{D} \text { : BPX-50 } 2 \mathrm{~m} \times 0.1 \mathrm{~mm} \times 0.1 \mu \mathrm{m} \\
\text { Modulator: ZX-2 LN2 cooled-loop thermal modulator }\end{array}$ & Screening & [37] \\
\hline $\begin{array}{l}\text { Wastewater, } \\
\text { suspended } \\
\text { solids, sludge }\end{array}$ & Steroids & $\begin{array}{l}\text { SPE and } \\
\text { ultrasonication } \\
\text { Florisil clean-up } \\
\text { derivatization }\end{array}$ & $\begin{array}{l}\text { GC } \times \text { GC-TOF-MS } \\
{ }^{1} \mathrm{D}: \text { BGB-5MS } 30 \mathrm{~m} \times 0.25 \mathrm{~mm} \times 0.25 \mu \mathrm{m} \\
{ }^{2} \mathrm{D}: \mathrm{DB}-17 \mathrm{MS} 1 \mathrm{~m} \times 0.1 \mathrm{~mm} \times 0.1 \mu \mathrm{m}\end{array}$ & & {$[38]$} \\
\hline
\end{tabular}




\begin{tabular}{|c|c|c|c|c|c|}
\hline Application & Target analytes & Sample Preparation & Instrumentation & $\begin{array}{l}\text { Comments / Method } \\
\text { Performance }\end{array}$ & Reference \\
\hline & & & Modulation : $3 \mathrm{~s}$ & & \\
\hline River water & Odorants & LLE & $\begin{array}{l}\text { GC } \times \text { GC-TOF-MS } \\
{ }^{1} \text { D: Rxi-5silv } 30 \mathrm{~m} \times 0.25 \mathrm{~mm} \times 0.25 \mu \mathrm{m} \\
{ }^{2} \mathrm{D}: \text { Rxi-17 } 1.79 \mathrm{~m} \times 0.1 \mathrm{~mm} \times 0.1 \mu \mathrm{m} \\
\text { Modulation : } 5 \mathrm{~s}\end{array}$ & & [39] \\
\hline River water & $\begin{array}{l}55 \text { target compounds: } \\
13 \text { personal care products (PCPs) } \\
15 \text { PAHs } \\
27 \text { pesticides }\end{array}$ & $\begin{array}{l}\text { SBSE - thermal } \\
\text { desorption }\end{array}$ & $\begin{array}{l}\text { GC } \times \text { GC-TOF-MS } \\
{ }^{1} \mathrm{D}: \text { Rtx-5 } 10 \mathrm{~m} \times 0.18 \mathrm{~mm} \times 0.2 \mu \mathrm{m} \\
{ }^{2} \mathrm{D}: \text { Rxi-17 } 1 \mathrm{~m} \times 0.1 \mathrm{~mm} \times 0.1 \mu \mathrm{m} \\
\text { or } \\
{ }^{2} \mathrm{D}: \text { Rt-LC50 } 1 \mathrm{~m} \times 0.1 \mathrm{~mm} \times 0.1 \mu \mathrm{m}\end{array}$ & $\begin{array}{l}\text { LODs: } 0.02 \text { to } 2.5 \mathrm{ng} / \mathrm{L} \text { for } \\
\text { wastewater effluent } \\
\text { LODs: } 0.01 \text { to } 2.15 \mathrm{ng} / \mathrm{L} \text { for river } \\
\text { water } \\
\text { LODs: } 3 \text { to } 10.2 \mathrm{ng} / \mathrm{L} \text { for more } \\
\text { polar analytes }\end{array}$ & [40] \\
\hline $\begin{array}{l}\text { Surface water, } \\
\text { soil, sediments }\end{array}$ & $\begin{array}{l}\text { Multi-class : 100-500 compounds } \\
\text { detected }\end{array}$ & $\begin{array}{l}\text { Water: LLE } \\
\text { Sediment/soil: } \\
\text { ultrasonic extraction } \\
\text { and GPC clean-up }\end{array}$ & $\begin{array}{l}\text { GC } \times \text { GC-TOF-MS } \\
{ }^{1} \mathrm{D}: \text { DB-XLB } 30 \mathrm{~m} \times 0.25 \mathrm{~mm} \times 0.25 \mu \mathrm{m} \\
{ }^{2} \mathrm{D}: \text { Rxi-17 } 1.5 \mathrm{~m} \times 0.15 \mathrm{~mm} \times 0.15 \mu \mathrm{m} \\
\text { Modulation : } 2 \mathrm{~s}\end{array}$ & $\begin{array}{l}\text { Quantitative and semiquantitative } \\
\text { LOQ : } 0.62-21\end{array}$ & [41] \\
\hline Surface water & $\begin{array}{l}\text { EDCs, emerging EDCs, and } \\
\text { antiretroviral drugs }\end{array}$ & $\begin{array}{l}\text { Disposable loop- } \\
\text { samplers (PDMS) }\end{array}$ & $\begin{array}{l}\text { GC } \times \text { GC-TOF-MS } \\
{ }^{1} \text { D: Rtx-CLPesticides II } 30 \mathrm{~m} \text { x } 0.25 \mathrm{~mm} \times 0.25 \mu \mathrm{m} \\
{ }^{2} \text { D: Rxi-17Sil MS } 1 \mathrm{~m} \text { x } 0.25 \mathrm{~mm} \text { x } 0.25 \mu \mathrm{m} \\
\text { Modulation : } 3 \mathrm{~s}\end{array}$ & $\begin{array}{l}\text { Quantitative and screening } \\
\text { Recoveries: } 85 \% \text { to } 121 \% \\
\text { (except for caffeine } 152 \% \text { ) } \\
\text { LODs: } 0.001 \mathrm{ng} / \mathrm{L} \text { to } 0.098 \mathrm{ng} / \mathrm{L} \\
\text { LOQs: } 0.003 \text { to } 0.33 \mathrm{ng} / \mathrm{L}\end{array}$ & [42] \\
\hline Wastewater & $\begin{array}{l}\text { Non-targeted analysis } \\
\text { Targeted: } 26 \text { compounds }\end{array}$ & LLE and Soxhlet & GC $\times$ GC-TOF-MS & $\begin{array}{l}\text { Quantitative and screening: } \\
\text { LOQs: } 3.3-49 \mathrm{pg} / \mu \mathrm{L}\end{array}$ & [43] \\
\hline
\end{tabular}




\begin{tabular}{|c|c|c|c|c|c|}
\hline Application & Target analytes & Sample Preparation & Instrumentation & $\begin{array}{l}\text { Comments / Method } \\
\text { Performance }\end{array}$ & Reference \\
\hline & & $\begin{array}{l}\text { SPE and } \\
\text { ultrasonication }\end{array}$ & $\begin{array}{l}{ }^{1} \mathrm{D}: \mathrm{BPX}-5029.5 \mathrm{~m} \times 0.25 \mathrm{~mm} \times 0.25 \mu \mathrm{m} \\
{ }^{2} \mathrm{D} \text { : VF-1 MS } 1.2 \mathrm{~m} \times 0.15 \mathrm{~mm} \times 0.15 \mu \mathrm{m} \\
\text { Modulation : } 3 \mathrm{~s}\end{array}$ & MLOQs: 23-1300 ng/L & \\
\hline Water systems & $\begin{array}{l}\text { Carbamazepine (CBZ), } \\
\text { galaxolide (HHCB), caffeine } \\
(\mathrm{CAF}) \text {, tonalide (AHTN), 4- } \\
\text { nonylphenol } \\
\text { (NP), and bisphenol A (BPA) }\end{array}$ & $\begin{array}{l}\text { SPE: EmporeTM } \\
\text { Styrene } \\
\text { Divinyl Benzene } \\
\text { (SDB-RPS) disks }\end{array}$ & $\begin{array}{l}\text { GC } \times \text { GC-TOF-MS } \\
{ }^{1} \text { D: Rtx-5SilMS } 30 \mathrm{~m} \times 0.25 \mathrm{~mm} \times 0.25 \mu \mathrm{m} \\
{ }^{2} \mathrm{D}: \text { Rxi-17Sil MS } 1 \mathrm{~m} \times 0.25 \mathrm{~mm} \times 0.25 \mu \mathrm{m} \\
\text { Modulation : } 3 \mathrm{~s}\end{array}$ & $\begin{array}{l}\text { Quantitative: } \\
\text { LOD: } 0.39 \text { to } 1.1 \mathrm{ng} / \mathrm{L} \\
\text { LOQs: } 1.2 \text { to } 3.9 \mathrm{ng} / \mathrm{L}\end{array}$ & [44] \\
\hline Wastewater & $\begin{array}{l}\text { Drugs, personal care products, } \\
\text { pesticides, carcinogens and } \\
\text { compounds toxic for } \\
\text { reproduction were identified }\end{array}$ & $\begin{array}{l}\text { Extraction: LL } \\
\text { No clean-up }\end{array}$ & $\begin{array}{l}\text { GC×GC-TOFMS } \\
{ }^{1} \mathrm{D}: \text { HP-5 30m x } 0.25 \mathrm{~mm} \times 0.32 \mu \mathrm{m} \\
{ }^{2} \mathrm{D}: \text { MegaWaxHT } 1 \mathrm{~m} \times 0.1 \mathrm{~mm} \times 0.1 \mu \mathrm{m} \\
\text { Modulation: } 6 \mathrm{sec}\end{array}$ & $\begin{array}{l}\text { Screening method / semi- } \\
\text { quantitative approach }\end{array}$ & [149] \\
\hline $\begin{array}{l}\text { Effluent and } \\
\text { river water }\end{array}$ & Multiresidue monitoring & SBSE & $\begin{array}{l}\text { GC } \times \text { GC-TOFMS } \\
{ }^{1} \mathrm{D}: \mathrm{Rtx}-510 \mathrm{~m} \times 0.18 \mathrm{~mm} \times 0.2 \mu \mathrm{m} \\
{ }^{2} \mathrm{D}: \mathrm{Rxi}-171 \mathrm{~m} \times 0.1 \mathrm{~mm} \times 0.1 \mu \mathrm{m}\end{array}$ & Quantitative and screening & [150] \\
\hline River water & $\begin{array}{l}\text { Odour-causing compounds (54 } \\
\text { compounds) }\end{array}$ & Extraction: LL & $\begin{array}{l}\text { GC } \times \text { GC-TOFMS } \\
\text { 'D: Rxi-5silv } 30 \mathrm{~m} \times 0.25 \mathrm{~mm} \times 0.32 \mu \mathrm{m} \\
{ }^{2} \mathrm{D}: \text { Rxi-17 } 1.79 \mathrm{~m} \times 0.1 \mathrm{~mm} \times 0.1 \mu \mathrm{m} \\
\text { Modulation: } 5 \mathrm{sec}\end{array}$ & $\begin{array}{l}\text { Quantitative: } \\
\text { LOD: 0.02-20.95 ng/L } \\
\text { LOQ: 0.05-70.55 ng/L }\end{array}$ & [151] \\
\hline
\end{tabular}




\begin{tabular}{|c|c|c|c|c|c|}
\hline Application & Target analytes & Sample Preparation & Instrumentation & $\begin{array}{l}\text { Comments / Method } \\
\text { Performance }\end{array}$ & Reference \\
\hline $\begin{array}{l}\text { Landfill } \\
\text { leachate }\end{array}$ & VOCs & SPME & $\begin{array}{l}\text { GC } \times \text { GC-qMS } \\
{ }^{1} \text { D: VF-1 } 30 \mathrm{~m} \times 0.25 \mathrm{~mm} \times 0.32 \mu \mathrm{m} \\
{ }^{2} \mathrm{D}: \text { DB-1701 } 1.5 \mathrm{~m} \text { x } 0.1 \mathrm{~mm} \times 0.1 \mu \mathrm{m} \\
\text { Modulation: } 8 \mathrm{sec}\end{array}$ & $\begin{array}{l}\text { Quantitative: } \\
\text { LOD: } 0.02-5.4 \mathrm{ng} / \mathrm{mL} \\
\text { LOQ: } 0.07-17.94 \mathrm{ng} / \mathrm{mL}\end{array}$ & [152] \\
\hline Water & Disinfection by-products & $\begin{array}{l}\text { Extraction: SPE } \\
\text { Supelclean LC-C18 } \\
\text { coupled with Oasis } \\
\text { HLB cartridge }\end{array}$ & $\begin{array}{l}\text { GC } \times \text { GC-qFMS } \\
{ }^{1} \mathrm{D} \text { : DB5-MS } 30 \mathrm{~m} \times 0.25 \mathrm{~mm} \times 0.32 \mu \mathrm{m} \\
{ }^{2} \mathrm{D}: \mathrm{BPX}-502 \mathrm{~m} \times 0.1 \mathrm{~mm} \times 0.1 \mu \mathrm{m} \\
\text { Modulation: } 8 \mathrm{sec} \\
\text { Thermal modulator }\end{array}$ & Screening & [108] \\
\hline
\end{tabular}


Table A.2 - Recent applications of GC $\times$ GC for the analysis of biota samples

\begin{tabular}{|c|c|c|c|c|c|}
\hline Matrix & Target analytes & Sample Preparation & Analysis & Comments / Method Performance & Reference \\
\hline Separation & РCB & & $\begin{array}{l}\text { GC } \times \text { GC-TOFMS } \\
{ }^{1} \text { D: SPB-Octyl } 30 \mathrm{~m} \times 0.25 \mathrm{~mm} \times 0.25 \mu \mathrm{m} \\
{ }^{2} \text { D: SLB-IL59 } 1.8 \mathrm{~m} \times 0.18 \mathrm{~mm} \times 0.18 \mu \mathrm{m} \\
\text { Modulation: } 3.5 \mathrm{~s}\end{array}$ & $\begin{array}{l}\text { Qualitative: enhanced PCB } \\
\text { separation }\end{array}$ & [46] \\
\hline $\begin{array}{l}\text { Marine tissue: } \\
\text { whiting and } \\
\text { guillemot liver } \\
\text { sample }\end{array}$ & РCB & $\begin{array}{l}\text { Sonication and } \\
\text { extraction with } \\
\text { dichloromethane/meth } \\
\text { anol } \\
\text { Clean-up: silica }\end{array}$ & $\begin{array}{l}\text { GC×GC-TOFMS } \\
{ }^{1} \text { D: Rtx-PCB } 60 \mathrm{~m} \text { x } 0.25 \mathrm{~mm} \times 0.25 \mu \mathrm{m} \\
{ }^{2} \mathrm{D}: \text { Rxi-17 } 1.5 \mathrm{~m} \text { x } 0.1 \mathrm{~mm} \text { x } 0.1 \mu \mathrm{m} \\
\text { Modulation: } 3.0 \mathrm{~s}\end{array}$ & Qualitative & [47] \\
\hline $\begin{array}{l}\text { Fat samples } \\
\text { (polar bear) }\end{array}$ & $\begin{array}{l}\text { POPs: PCBs, PCDD/Fs, PBDEs, } \\
\text { OCPs, PCNs, PCTs and CTT }\end{array}$ & $\begin{array}{l}\text { Fat dissolved in n- } \\
\text { hexane } \\
\text { Eluted though a } \\
\text { multilayer silica }\end{array}$ & 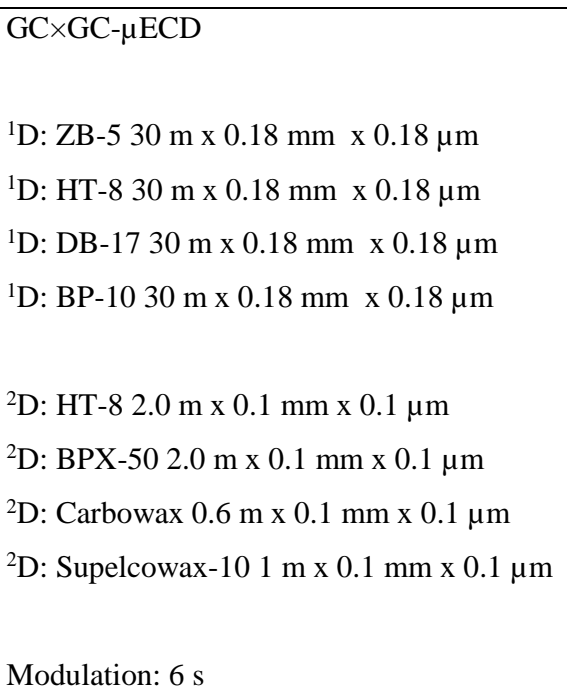 & Qualitative: group type separation & [49] \\
\hline
\end{tabular}




\begin{tabular}{|c|c|c|c|c|c|}
\hline Matrix & Target analytes & Sample Preparation & Analysis & Comments / Method Performance & Reference \\
\hline Fish & PAHs, PCBs, BDEs & $\begin{array}{l}\text { Extraction: ethyl } \\
\text { acetate } \\
\text { Clean-up: silica }\end{array}$ & $\begin{array}{l}\text { LV-GC×GC-TOFMS } \\
{ }^{1} \mathrm{D}: \mathrm{BPX}-530 \mathrm{~m} \times 0.25 \mathrm{~mm} \times 0.25 \mu \mathrm{m} \\
{ }^{2} \mathrm{D}: \text { BPX-50 } 1 \mathrm{~m} \times 0.1 \mathrm{~mm} \times 0.1 \mu \mathrm{m} \\
\text { Modulation: } 4 \mathrm{~s}\end{array}$ & $\begin{array}{l}\text { LOQs: } \\
\text { PCBs: } 0.01-0.25 \mu \mathrm{g} / \mathrm{kg} \\
\text { PBDEs: } 0.025-5 \mu \mathrm{g} / \mathrm{kg} \\
\text { PAHs: } 0.025-0.5 \mu \mathrm{g} / \mathrm{kg} \\
\text { \%Recoveries: } \\
\text { PCBs: } 75-120 \% \\
\text { PBDEs: } 91-117 \% \\
\text { PAHs: } 78-97 \%\end{array}$ & [50] \\
\hline Mussels & $\begin{array}{l}\text { isomeric branched alkyl } \\
\text { benzenes (BAB) } \\
\text { alkyl indanes (BIN) } \\
\text { branched alkyl tetralins (BAT) } \\
\text { aromatic hydrocarbon }\end{array}$ & $\begin{array}{l}\text { Alkaline } \\
\text { saponification method } \\
\text { digests extracted with } \\
\text { n-hexane }\end{array}$ & $\begin{array}{l}\text { GC-MSD } \\
\text { HP1-MS } 30 \mathrm{~m} \times 0.25 \mathrm{~mm} \times 0.25 \mu \mathrm{m} \\
\text { GC } \times \text { GC-TOFMS } \\
{ }^{1} \mathrm{D}: \text { DB-5 } 30 \mathrm{~m} \times 0.18 \mathrm{~mm} \times 0.18 \mu \mathrm{m} \\
{ }^{2} \mathrm{D}: \text { DB- } 171.9 \mathrm{~m} \times 0.1 \mathrm{~mm} \times 0.1 \mu \mathrm{m} \\
\text { Modulation: } 4 \mathrm{~s}\end{array}$ & Qualitative & [51] \\
\hline Fish oil & POPs & $\begin{array}{l}\text { GPC }-1^{\text {st }} \text { clean-up } \\
\text { followed by GPC or } \\
\text { SPE or acidification }\end{array}$ & $\begin{array}{l}\text { GC } \times \text { GC-TOFMS } \\
{ }^{1} \text { D: Rtx-Dioxin2 } 60 \mathrm{~m} \text { x } 0.25 \mathrm{~mm} \times 0.25 \mu \mathrm{m} \\
{ }^{2} \mathrm{D}: \text { Rtx-PCB } 2.0 \mathrm{~m} \text { x } 0.18 \mathrm{~mm} \text { x } 0.18 \mu \mathrm{m} \\
{ }^{1} \text { DRtx-5Sil-MS } 15 \mathrm{~m} \times 0.25 \mathrm{~mm} \times 0.25 \mu \mathrm{m} \\
{ }^{2} \mathrm{D}: \text { DB-17MS } 2.0 \mathrm{~m} \times 0.18 \mathrm{~mm} \times 0.18 \mu \mathrm{m}\end{array}$ & Targeted and non-targeted & {$[52,157]$} \\
\hline Fish oil & $\begin{array}{l}\text { POPs: } 15 \text { PCBs, PBDEs, } \\
\text { octachlorostyrene, MEO-PBDEs, }\end{array}$ & GPC & GC $\times$ GC-TOFMS & LODs: $0.44-0.63 \mathrm{pg} / \mathrm{g}$ & [53] \\
\hline
\end{tabular}




\begin{tabular}{|c|c|c|c|c|c|}
\hline Matrix & Target analytes & Sample Preparation & Analysis & Comments / Method Performance & Reference \\
\hline & $\begin{array}{l}\text { OCPs, HCB, oxybenzone, } \\
\text { dibromoindole }\end{array}$ & & $\begin{array}{l}{ }^{1} \mathrm{D} \text { : Rtx-5Sil-MS } 15 \mathrm{~m} \times 0.25 \mathrm{~mm} \times 0.25 \mu \mathrm{m} \\
{ }^{2} \mathrm{D} \text { : DB-17MS } 2.0 \mathrm{~m} \times 0.18 \mathrm{~mm} \times 0.18 \mu \mathrm{m} \\
\text { Modulation: } 3.5 \mathrm{~s}\end{array}$ & & \\
\hline Dolphins & 271 compounds & $\begin{array}{l}\text { Blubber } \\
\text { homogenization and } \\
\text { filtration } \\
\text { GPC }\end{array}$ & $\begin{array}{l}\text { GC } \times \text { GC-TOFMS } \\
{ }^{1} \text { D: Rtx-5Sil-MS } 15 \mathrm{~m} \text { x } 0.25 \mathrm{~mm} \times 0.25 \mu \mathrm{m} \\
{ }^{2} \mathrm{D}: \text { DB-17MS } 2.0 \mathrm{~m} \text { x } 0.18 \mathrm{~mm} \times 0.18 \mu \mathrm{m} \\
\text { Modulation: } 3.5 \mathrm{~s}\end{array}$ & Non-target analysis & [54] \\
\hline Dolphins & $327 \mathrm{HOC}$ & $\begin{array}{l}\text { PLE } \\
\text { Clean-up: GPC }\end{array}$ & $\begin{array}{l}\text { GC } \times \text { GC-TOFMS } \\
{ }^{1} \text { D: Rtx-5Sil-MS } 15 \mathrm{~m} \times 0.25 \mathrm{~mm} \times 0.25 \mu \mathrm{m} \\
{ }^{2} \mathrm{D}: \text { DB-17MS } 2.0 \mathrm{~m} \text { x } 0.18 \mathrm{~mm} \times 0.18 \mu \mathrm{m} \\
\text { Modulation: } 3.5 \mathrm{~s}\end{array}$ & $\begin{array}{l}\text { Qualitative } \\
\text { Mass spectral libraries }\end{array}$ & [55] \\
\hline $\begin{array}{l}\text { Marine tissue: } \\
\text { Bluefin tuna }\end{array}$ & $\begin{array}{l}\text { Organobromines: PBDEs, PBBs, } \\
\text { MeO-PBDEs, HNPs, novel BFRs, } \\
\text { PBHDs, tribromoanisole and a } \\
\text { mixed halogenated compound }\end{array}$ & $\begin{array}{l}\text { Soxhlet } \\
\text { Clean-up: acid silica }\end{array}$ & $\begin{array}{l}\text { GC } \times \text { GC-TOFMS } \\
{ }^{1} \text { D: HT- } 830 \mathrm{~m} \times 0.25 \mathrm{~mm} \times 0.25 \mu \mathrm{m} \\
{ }^{2} \mathrm{D}: \text { BPX-50 } 1.6 \mathrm{~m} \times 0.1 \mathrm{~mm} \times 0.1 \mu \mathrm{m} \\
\text { Modulation: } 6 \mathrm{~s} \\
\text { GC-MS/MS for confirmation } \\
\text { HP-5MS } 30 \mathrm{~m} \times 0.25 \mathrm{~mm} \times 0.25 \mu \mathrm{m}\end{array}$ & Qualitative & $[56,59]]$ \\
\hline Seabirds & $111 \mathrm{HOC}$ & $\begin{array}{l}\text { Extraction: acid } \\
\text { digestion and liquid- } \\
\text { liquid extraction }\end{array}$ & $\begin{array}{l}\text { GC } \times \text { GC-TOFMS } \\
{ }^{1} \mathrm{D}: \text { Rtx-5Sil-MS } 15 \mathrm{~m} \text { x } 0.25 \mathrm{~mm} \times 0.25 \mu \mathrm{m}\end{array}$ & Qualitative & [57] \\
\hline
\end{tabular}




\begin{tabular}{|c|c|c|c|c|c|}
\hline Matrix & Target analytes & Sample Preparation & Analysis & Comments / Method Performance & Reference \\
\hline & & Clean-up: GPC & $\begin{array}{l}{ }^{2} \mathrm{D}: \text { DB-17MS } 2.0 \mathrm{~m} \text { x } 0.18 \mathrm{~mm} \times 0.18 \mu \mathrm{m} \\
\text { Modulation: } 3.5 \mathrm{~s}\end{array}$ & & \\
\hline Fats and oils & PCBs, PBDEs, BFRs, PAHs & $\begin{array}{l}\text { Sample dilution in } \\
\text { hexane } \\
\text { Clean-up: Silica }\end{array}$ & $\begin{array}{l}\text { GC×GC-TOFMS } \\
{ }^{1} \text { D: Rxi-17Sil-MS } 30 \mathrm{~m} \text { x } 0.25 \mathrm{~mm} \times 0.25 \mu \mathrm{m} \\
{ }^{2} \mathrm{D}: \text { Rxi-5Sil-MS } 1.0 \mathrm{~m} \text { x } 0.18 \mathrm{~mm} \times 0.18 \mu \mathrm{m} \\
\text { Modulation: } 3 \mathrm{~s}\end{array}$ & Qualitative & [58] \\
\hline Fish & PCBs, PCNs & $\begin{array}{l}\text { EPA } 1668 \\
\text { Extraction: ASE } \\
\text { Clean-up: silica and } \\
\text { alumina columns }\end{array}$ & $\begin{array}{l}\text { GC } \times \text { GC-HRTOFMS } \\
{ }^{1} \mathrm{D}: \text { DB-XLB } 20 \mathrm{~m} \text { x } 0.25 \mathrm{~mm} \times 0.25 \mu \mathrm{m} \\
{ }^{2} \mathrm{D}: \text { BPX-70 } 2.0 \mathrm{~m} \text { x } 0.1 \mathrm{~mm} \times 0.1 \mu \mathrm{m} \\
\text { Modulation: } 10 \mathrm{~s}\end{array}$ & $\begin{array}{l}\text { LODs: PCBs: } 0.03-0.3 \mathrm{pg} / \mu \mathrm{L} \\
\text { PCNs: } 0.09-0.6 \mathrm{pg} / \mu \mathrm{L} \\
\text { MDLs: PCBs: } 0.6-3.5 \mathrm{pg} / \mathrm{g} \\
\text { PCNs: } 0.8-4.6 . \mathrm{pg} / \mathrm{g} \\
\text { Recoveries: PCBs: } 53-114 \% \\
\text { PCNs: } 47-121 \%\end{array}$ & [60] \\
\hline Fish & Lipids & $\begin{array}{l}\text { Extraction: } \\
\text { chloroform:methanol } \\
\text { Derivatization }\end{array}$ & $\begin{array}{l}\text { GC } \times \text { GC-MS } \\
{ }^{1} \text { D: SLB-5 ms } 30 \mathrm{~m} \text { x } 0.25 \mathrm{~mm} \times 0.25 \mu \mathrm{m} \\
{ }^{2} \mathrm{D} \text { : Supelcowax-10 } 1.5 \mathrm{~m} \times 0.1 \mathrm{~mm} \times 0.1 \mu \mathrm{m} \\
\text { Modulator: ZOEX }\end{array}$ & Qualitative & [89] \\
\hline Fish & $\begin{array}{l}72 \text { compounds: } 33 \text { PCBs, } 9 \text { OCPs, } \\
4 \\
\text { PBDEs, } 4 \text { metabolites of DDT, } 2 \\
\text { chlorinated anisole, } 2 \\
\text { chlorostyrene, } 1 \text { thioanisole, and } \\
\text { methyl triclosan }\end{array}$ & $\begin{array}{l}\text { Soxhlet extraction } \\
\text { Clean-up: GPC }\end{array}$ & $\begin{array}{l}\text { GC } \times \text { GC-TOFMS } \\
{ }^{1} \text { D: DB-5MS } 30 \mathrm{~m} \times 0.25 \mathrm{~mm} \times 0.25 \mu \mathrm{m} \\
{ }^{2} \mathrm{D}: \text { HT-8 } 1.2 \mathrm{~m} \times 0.1 \mathrm{~mm} \times 0.1 \mu \mathrm{m} \\
\text { Modulation: } 4 \mathrm{~s}\end{array}$ & Qualitative & [153] \\
\hline
\end{tabular}




\begin{tabular}{|c|c|c|c|c|c|}
\hline Matrix & Target analytes & Sample Preparation & Analysis & Comments / Method Performance & Reference \\
\hline Mussels & Mixed halogenated: PXDD/Fs & $\begin{array}{l}\text { Freeze dried tissue } \\
\text { Extraction: } \\
\text { acetone/hexane } \\
\text { Clean-up: acid, silica }\end{array}$ & $\begin{array}{l}\text { GC } \times \text { GC-HRTOFMS } \\
{ }^{1} \mathrm{D}: \text { BPX-5 } 30 \mathrm{~m} \times 0.25 \mathrm{~mm} \times 0.25 \mu \mathrm{m} \\
{ }^{2} \mathrm{D}: \text { BPX-50 } 2.0 \mathrm{~m} \times 0.1 \mathrm{~mm} \times 0.1 \mu \mathrm{m}\end{array}$ & Quantitative and screening & [154] \\
\hline
\end{tabular}


Table A.3. - Recent applications of GC $\times$ GC for the analysis of soil/sediment/sludge samples

\begin{tabular}{|c|c|c|c|c|c|}
\hline Matrix & Target analytes & Sample Preparation & Analysis & Comments & Reference \\
\hline Sediments & PAHs & $\begin{array}{l}\text { Extraction: MAE } \\
\text { Clean-up: SPE }\end{array}$ & $\begin{array}{l}\text { GC } \times \text { GC-TOFMS } \\
{ }^{1} \text { D: DB-5 } 30 \mathrm{~m} \times 0.25 \mathrm{~mm} \times 0.25 \mu \mathrm{m} \\
{ }^{2} \text { D: BPX-50 } 1.6 \mathrm{~m} \times 0.1 \mathrm{~mm} \times 0.1 \mu \mathrm{m} \\
{ }^{1} \text { D: HT- } 830 \mathrm{~m} \times 0.25 \mathrm{~mm} \times 0.25 \mu \mathrm{m} \\
{ }^{2} \text { D: BPX-50 } 1.6 \mathrm{~m} \times 0.1 \mathrm{~mm} \times 0.1 \mu \mathrm{m} \\
\text { Modulation: } 5 \mathrm{~s}\end{array}$ & LODs: $5.7-60 \mu \mathrm{g} / \mathrm{kg}$ & [61] \\
\hline Soil & $\begin{array}{l}\text { Cl-/Br-PAHs } \\
30 \mathrm{Cl}-/ \mathrm{Br}-\mathrm{PAHs} \\
10 \mathrm{PAHs}, \mathrm{ClBr}-\mathrm{PAHs} \\
35 \text { other organohalogens }\end{array}$ & $\begin{array}{l}\text { Extraction: Soxhlet } \\
\text { No clean-up }\end{array}$ & $\begin{array}{l}\text { GC×GC-HRTOF-MS } \\
{ }^{1} \text { D: BPX-5 } 30 \mathrm{~m} \text { x } 0.25 \mathrm{~mm} \times 0.25 \mu \mathrm{m} \\
{ }^{2} \mathrm{D}: \text { BPX-50 } 1.0 \mathrm{~m} \times 0.1 \mathrm{~mm} \times 0.1 \mu \mathrm{m} \\
\text { Modulation: } 4 \mathrm{~s} \\
{ }^{1} \mathrm{D} \text { : BPX-5 } 30 \mathrm{~m} \times 0.25 \mathrm{~mm} \times 0.25 \mu \mathrm{m} \\
{ }^{2} \mathrm{D} \text { : LC-50HT } 1.0 \mathrm{~m} \times 0.1 \mathrm{~mm} \times 0.1 \mu \mathrm{m} \\
\text { Modulation: } 8 \mathrm{~s}\end{array}$ & $\begin{array}{l}\text { LODs: } \\
\text { Cl-PAHs } 0.08-0.44 \mathrm{pg} \\
\text { Br-PAHs } 0.26 \text { pg to } 3.2 \mathrm{pg}\end{array}$ & [62] \\
\hline Soil & Halogenated PAHs & $\begin{array}{l}\text { Extraction: ASE } \\
\text { Clean-up: alumina } \\
\text { column }\end{array}$ & $\begin{array}{l}\text { FT-ICR } \\
\text { GC×GC-HRTOFMS } \\
{ }^{1} \text { D:DB-5HT } 15 \mathrm{~m} \times 0.25 \mathrm{~mm} \times 0.25 \mu \mathrm{m} \\
{ }^{2} \mathrm{D}: \text { Rtx-50 } 2.3 \mathrm{~m} \times 0.18 \mathrm{~mm} \times 0.18 \mu \mathrm{m} \\
\text { Modulation: } 4 \mathrm{~s}\end{array}$ & $\begin{array}{l}\text { Screening : approx. } 150 \text { formulas } \\
\text { identified }\end{array}$ & [63] \\
\hline
\end{tabular}




\begin{tabular}{|c|c|c|c|c|c|}
\hline Matrix & Target analytes & Sample Preparation & Analysis & Comments & Reference \\
\hline & & & $\begin{array}{l}\text { GC } \times \text { GC-TOFMS } \\
{ }^{1} \text { D:DB-17HT } 30 \mathrm{~m} \times 0.25 \mathrm{~mm} \times 0.15 \mu \mathrm{m} \\
{ }^{2} \mathrm{D}: \text { DB-5MS } 1 \mathrm{~m} \times 0.1 \mathrm{~mm} \times 0.1 \mu \mathrm{m} \\
\text { Modulation: } 5 \mathrm{~s}\end{array}$ & & \\
\hline $\begin{array}{l}\text { Dust - } \\
\text { electronic } \\
\text { waste facility }\end{array}$ & Multi-class & Sonication & $\begin{array}{l}\text { GC } \times \text { GC-HRTOFMS using EI and NCI } \\
{ }^{1} \text { D: Rxi-5SilMS } 30 \mathrm{~m} \times 0.25 \mathrm{~mm} \times 0.25 \mu \mathrm{m} \\
{ }^{2} \text { D: Rxi-17SilMS } 2 \mathrm{~m} \times 0.15 \mathrm{~mm} \times 0.15 \mu \mathrm{m} \\
\text { Modulation: } 8 \mathrm{~s}\end{array}$ & Non-targeted analysis & [65] \\
\hline $\begin{array}{l}\text { Soil (coal tar } \\
\text { contaminated) } \\
\text {, bitumen tar } \\
\text { sand }\end{array}$ & $\begin{array}{l}\text { PAHs } \\
\text { S-PAHs }\end{array}$ & $\begin{array}{l}\text { Soil: sonication } \\
\text { Sand: microwave } \\
\text { extraction } \\
\text { Copper and } \mathrm{Na}_{2} \mathrm{SO}_{4} \\
\text { treatments }\end{array}$ & & $\begin{array}{l}\text { LODs: } 5.5 \text { to } 35.8 \mathrm{ng} / \mathrm{g} \\
\text { LOQs: } 10-50 \mathrm{ng} / \mathrm{mL}\end{array}$ & [66] \\
\hline $\begin{array}{l}\text { Fly ash, flue } \\
\text { gas extract }\end{array}$ & $\mathrm{Dx} / \mathrm{Fs}$ & & $\begin{array}{l}\text { GC } \times \text { GC-HRTOF-MS } \\
{ }^{1} \text { D: InertCap-5MS/Sil } 60 \mathrm{~m} \text { x } 0.25 \mathrm{~mm} \times 0.1 \mu \mathrm{m} \\
{ }^{2} \text { D: InertCap-17MS/Sil } 1.5 \mathrm{~m} \text { x } 0.075 \mathrm{~mm} \times 0.15 \mu \mathrm{m} \\
\text { Modulation: } 3 \mathrm{~s}\end{array}$ & $\begin{array}{l}\text { IDL: } 0.9 \text { to } 4 \mathrm{pg} \text { for chlorinated } \\
\text { dioxins and } 0.4 \text { to } 2 \mathrm{pg} \text { for } \\
\text { chlorinated furans }\end{array}$ & [67] \\
\hline $\begin{array}{l}\text { Fly ash } \\
\text { Sediment } \\
\text { Soil } \\
\text { Flue gas } \\
\text { extract }\end{array}$ & $\begin{array}{l}16 \text { PCDDs, } \\
19 \text { PCDFs, } 62 \text { PCBs, } 39 \text { PBDEs, } 21 \\
\text { chlorinated PAHs, } 11 \text { brominated } \\
\text { PAHs and } 43 \text { other POPs }\end{array}$ & $\begin{array}{l}\text { Soxhlet } \\
\text { Acid silica clean-up }\end{array}$ & $\begin{array}{l}\text { GC } \times \text { GC-MS/MS } \\
{ }^{1} \text { D: InertCap-5MS/Sil } 60 \mathrm{~m} \times 0.25 \mathrm{~mm} \times 0.1 \mu \mathrm{m} \\
{ }^{2} \mathrm{D}: \text { BPX-50 } 1.5 \mathrm{~m} \times 0.1 \mathrm{~mm} \times 0.15 \mu \mathrm{m} \\
\text { Modulation: } 4 \mathrm{~s}\end{array}$ & & [68] \\
\hline
\end{tabular}




\begin{tabular}{|c|c|c|c|c|c|}
\hline Matrix & Target analytes & Sample Preparation & Analysis & Comments & Reference \\
\hline $\begin{array}{l}\text { Fly ash, soil, } \\
\text { sediment, the } \\
\text { atmosphere, } \\
\text { and human } \\
\text { urine }\end{array}$ & Organohalogens & & $\begin{array}{l}\text { GC } \times \text { GC-HRTOF-MS } \\
{ }^{1} \text { D: InertCap-5MS/Sil } 60 \mathrm{~m} \times 0.25 \mathrm{~mm} \times 0.1 \mu \mathrm{m} \\
{ }^{2} \mathrm{D}: \text { BPX-50 } 1.5 \mathrm{~m} \times 0.1 \mathrm{~mm} \times 0.1 \mu \mathrm{m} \\
\text { Modulation: } 4 \mathrm{~s}\end{array}$ & & [69] \\
\hline $\begin{array}{l}\text { Sediment } \\
\text { CRM }\end{array}$ & $\begin{array}{l}17 \mathrm{PCDD} / \mathrm{Fs} \\
24 \mathrm{PCBc}\end{array}$ & $\begin{array}{l}\text { ASE } \\
\text { No clean-up }\end{array}$ & $\begin{array}{l}\text { GC } \times \text { GC-HRTOF-MS and GC×GC-qMS } \\
{ }^{1} \text { D: InertCap-5MS/Sil } 60 \mathrm{~m} \times 0.25 \mathrm{~mm} \times 0.1 \mu \mathrm{m} \\
{ }^{2} \mathrm{D} \text { : SLB-IL59 } 1.5 \mathrm{~m} \times 0.1 \mathrm{~mm} \times 0.08 \mu \mathrm{m} \\
\text { Modulation: } 4 \mathrm{~s}\end{array}$ & $\begin{array}{l}\text { LOQs: } \\
73.7-1262 \mathrm{pg} / \mathrm{g} \text { for } 17 \mathrm{PCDD} / \mathrm{Fs} \\
7.1-54.2 \mathrm{pg} / \mathrm{g} \text { for } 12 \mathrm{PCB}\end{array}$ & [70] \\
\hline Sediment core & $\begin{array}{l}\text { Chlorinated compounds standards: } \\
35 \text { PCBs, } 43 \text { POPs, } 39 \text { BDEs, } 21 \\
\text { Cl- and } 11 \text { Br-PAHs } \\
\text { Other compounds identified: } \\
\text { PCDD/Fs, pesticides }\end{array}$ & $\begin{array}{l}\text { Extraction: ASE } \\
\text { Clean-up: SPE }\end{array}$ & $\begin{array}{l}\text { GC } \times \text { GC-HRTOF-MS and GC×GC-qMS } \\
{ }^{1} \mathrm{D}: \text { InertCap-5MS/Sil } 60 \mathrm{~m} \times 0.25 \mathrm{~mm} \times 0.1 \mu \mathrm{m} \\
{ }^{2} \mathrm{D}: \text { SLB-IL59 } 1.5 \mathrm{~m} \times 0.1 \mathrm{~mm} \times 0.08 \mu \mathrm{m} \\
\text { Modulation: } 4 \mathrm{~s}\end{array}$ & $\begin{array}{l}\text { Untargeted and targeted, semi- } \\
\text { quantitation }\end{array}$ & [71-72] \\
\hline $\begin{array}{l}\text { Sediments, } \\
\text { soil }\end{array}$ & PCDD/PCDF (17 compounds) & $\begin{array}{l}\text { Extraction: ASE } \\
\text { (EPA Method 1613) } \\
\text { Clean-up: GPC }\end{array}$ & $\begin{array}{l}\text { GC×GC-TOFMS } \\
{ }^{1} \text { D: Rtx-XLB } 30 \mathrm{~m} \text { x } 0.25 \mathrm{~mm} \times 0.25 \mu \mathrm{m} \\
{ }^{2} \mathrm{D}: \text { Rtx-200 } 2.0 \mathrm{~m} \text { x } 0.18 \mathrm{~mm} \times 0.2 \mu \mathrm{m} \\
{ }^{1} \mathrm{D} \text { : Rxi-5SilMS } 30 \mathrm{~m} \times 0.25 \mathrm{~mm} \times 0.25 \mu \mathrm{m} \\
{ }^{2} \mathrm{D}: \text { Rtx-200 } 2.0 \mathrm{~m} \times 0.18 \mathrm{~mm} \times 0.18 \mu \mathrm{m} \\
{ }^{1} \text { D: Rtx-Dioxin } 260 \mathrm{~m} \times 0.25 \mathrm{~mm} \times 0.25 \mu \mathrm{m} \\
{ }^{2} \mathrm{D}: \text { Rtx-PCB } 2.0 \mathrm{~m} \times 0.18 \mathrm{~mm} \times 0.18 \mu \mathrm{m}\end{array}$ & $\begin{array}{l}\text { LOD: } 300 \text { fg on column (meeting } \\
\text { US EPA Method } 1613 \text { criteria) }\end{array}$ & [73] \\
\hline
\end{tabular}




\begin{tabular}{|c|c|c|c|c|c|}
\hline Matrix & Target analytes & Sample Preparation & Analysis & Comments & Reference \\
\hline & & & Modulation: $4 \mathrm{~s}$ & & \\
\hline $\begin{array}{l}\text { Hazardous } \\
\text { waste } \\
\text { treatment } \\
\text { facility wastes }\end{array}$ & PCDD/PCDF, PCB, PAH & $\begin{array}{l}\text { Extraction: Soxhlet } \\
\text { Clean-up: multilayer } \\
\text { silica column }\end{array}$ & $\begin{array}{l}\text { GC } \times \text { GC-TOFMS } \\
{ }^{1} \text { D: Rtx-5Silms } 30 \mathrm{~m} \text { x } 0.25 \mathrm{~mm} \times 0.25 \mu \mathrm{m} \\
{ }^{2} \mathrm{D} \text { : Rtx-PCB or } 2.0 \mathrm{~m} \times 0.18 \mathrm{~mm} \times 0.18 \mu \mathrm{m} \\
{ }^{1} \text { D: Rtx-5 or Rtx-XLB } 30 \mathrm{~m} \text { x } 0.25 \mathrm{~mm} \times 0.25 \mu \mathrm{m} \\
{ }^{2} \text { D: Rtx- } 200 \text { or } 2.0 \mathrm{~m} \times 0.18 \mathrm{~mm} \times 0.18 \mu \mathrm{m} \\
\text { Modulation: } 5 \mathrm{~s}\end{array}$ & Quantitative and qualitative & [74] \\
\hline Sediments & 2,3,7,8-PCDD/F & $\begin{array}{l}\text { Extraction: ASE } \\
\text { Clean-up: acid wash, } \\
\text { GPC, SPE }\end{array}$ & $\begin{array}{l}\text { GC } \times \text { GC-HRTOFMS using EI and NCI } \\
{ }^{1} \text { D: SGE BPX-5 } 15 \mathrm{~m} \times 0.25 \mathrm{~mm} \times 0.25 \mu \mathrm{m} \\
{ }^{2} \mathrm{D}: \text { HP-INNOWax } 3 \mathrm{~m} \times 0.25 \mathrm{~mm} \times 0.1 \mu \mathrm{m} \\
\text { Modulation: } 4 \mathrm{~s}\end{array}$ & & [75] \\
\hline $\begin{array}{l}\text { Sediments, } \\
\text { soil, sludges }\end{array}$ & $\begin{array}{l}118 \text { compounds } \\
\mathrm{PCB}, \mathrm{OC}, \mathrm{CB}\end{array}$ & $\begin{array}{l}\text { Extraction: ASE } \\
\text { Clean-up: SPE }\end{array}$ & $\begin{array}{l}\text { GC } \times \text { GC-TOFMS } \\
{ }^{1} \text { D: DB-1 } 30 \mathrm{~m} \times 0.25 \mathrm{~mm} \times 0.25 \mu \mathrm{m} \\
{ }^{2} \text { D: Rtx-PCB } 1.6 \mathrm{~m} \times 0.1 \mathrm{~mm} \times 0.1 \mu \mathrm{m} \\
\text { Modulation: } 4 \mathrm{~s}\end{array}$ & $\begin{array}{l}\text { Quantitative } \\
\text { MDL: } 0.1 \text { to } 1.6 \mathrm{ng} / \mathrm{g} \text { for PCBs, } \\
0.14 \text { to } 0.83 \mathrm{ng} / \mathrm{g} \text { for } \mathrm{CBs} \text { and } \\
0.12 \text { to } 0.50 \mathrm{ng} / \mathrm{g} \text { for } \mathrm{OCs}\end{array}$ & [28,76-77] \\
\hline Sediments & $\begin{array}{l}7 \text { pesticides: } \\
\text { propanil, fipronil, propiconazole, } \\
\text { trifloxystrobin, } \\
\text { permethrin, difenoconazole, } \\
\text { azoxystrobin }\end{array}$ & Extraction: sonication & $\begin{array}{l}\text { GC } \times \text { GC-TOFMS } \\
{ }^{1} \text { D: Rtx-5 } 30 \mathrm{~m} \times 0.25 \mathrm{~mm} \times 0.25 \mu \mathrm{m} \\
{ }^{2} \mathrm{D}: \text { DB-17 } 1.7 \mathrm{~m} \times 0.18 \mathrm{~mm} \times 0.18 \mu \mathrm{m} \\
{ }^{1} \mathrm{D}: \mathrm{HP}-50+30 \mathrm{~m} \times 0.25 \mathrm{~mm} \times 0.25 \mu \mathrm{m} \\
{ }^{2} \mathrm{D}: \text { DB-1MS } 1.7 \mathrm{~m} \times 0.1 \mathrm{~mm} \times 0.1 \mu \mathrm{m}\end{array}$ & $\begin{array}{l}\text { LODs: } 0.08 \text { to } 1.07 \mu \mathrm{g} / \mathrm{L} \\
\text { LOQ: } 0.25 \text { to } 3.23 \mu \mathrm{g} / \mathrm{L}\end{array}$ & [78] \\
\hline
\end{tabular}




\begin{tabular}{|c|c|c|c|c|c|}
\hline Matrix & Target analytes & Sample Preparation & Analysis & Comments & Reference \\
\hline & & & Modulation: $7 \mathrm{~s}$ & & \\
\hline Soil and air & $\begin{array}{l}\text { Enantiomeric } \\
\text { analysis of o,p'-DDT and o,p'- } \\
\text { DDD }\end{array}$ & PDMS & $\begin{array}{l}\text { GC-FID for off line heart-cut } \\
\text { ZB-1 } 30 \mathrm{~m} \times 0.25 \mathrm{~mm} \times 0.25 \mu \mathrm{m} \\
\text { Followed by: } \\
\text { GC-TOFMS for chiral separation, no modulation } \\
{ }^{1} \mathrm{D}: \text { BGB-15 } 30 \mathrm{~m} \times 0.25 \mathrm{~mm} \times 0.25 \mu \mathrm{m} \\
{ }^{2} \mathrm{D}: \text { Rtx-200 } 1.29 \mathrm{~m} \times 0.18 \mathrm{~mm} \times 0.18 \mu \mathrm{m} \\
\text { GC×GC-TOFMS } \\
{ }^{1} \mathrm{D}: \text { BGB-15 } 30 \mathrm{~m} \times 0.25 \mathrm{~mm} \times 0.25 \mu \mathrm{m} \\
{ }^{2} \mathrm{D}: \text { Rtx-200 } 1.29 \mathrm{~m} \times 0.18 \mathrm{~mm} \times 0.18 \mu \mathrm{m} \\
\text { Modulation : } 4 \mathrm{~s}\end{array}$ & & [81] \\
\hline Soil & Pesticides & QuEChERS & $\begin{array}{l}\text { GC } \times \text { GC-TOFMS } \\
{ }^{1} \text { D:Rtx-CL } 30 \mathrm{~m} \times 0.25 \mathrm{~mm} \times 0.25 \mu \mathrm{m} \\
{ }^{2} \mathrm{D}: \text { BPX-50 } 2 \mathrm{~m} \text { x } 0.1 \mathrm{~mm} \times 0.07 \mu \mathrm{m} \\
\text { Modulation: } 4.5 \mathrm{~s}\end{array}$ & MS/MS quantitation & [82] \\
\hline Soil & Toxaphenes: P26, P50 and P62 & $\begin{array}{l}\text { ASE } \\
\text { Clean-up: multilayer } \\
\text { acid silica and silica } \\
\text { packed columns }\end{array}$ & $\begin{array}{l}\text { GC } \times \text { GC-NCI-MS } \\
{ }^{1} \text { D: DB-XLB } 30 \mathrm{~m} \times 0.25 \mathrm{~mm} \times 0.15 \mu \mathrm{m} \\
{ }^{2} \mathrm{D}: \text { BPX-50 } 2 \mathrm{~m} \times 0.1 \mathrm{~mm} \times 0.1 \mu \mathrm{m} \\
\text { Modulation: } 6 \mathrm{~s}\end{array}$ & $\begin{array}{l}\text { LOD: } 0.6 \text { to } 1.0 \mathrm{pg} / \mathrm{g} \\
\text { LOQ: } 1.2 \text { to } 2.9 \mathrm{pg} / \mathrm{g} \\
\text { Recoveries: } 60-113 \%\end{array}$ & [83] \\
\hline Soil & $\begin{array}{l}37 \text { compounds, } \\
\text { Organophosphorus pesticides } \\
\text { organophosphate esters }\end{array}$ & $\begin{array}{l}\text { Extraction: sonication } \\
\text { Clean-up: SPE }\end{array}$ & $\begin{array}{l}\text { GC } \times \text { GC-FPD (P-mode) } \\
{ }^{1} \text { D: HP-5 } 30 \mathrm{~m} \times 0.32 \mathrm{~mm} \times 0.25 \mu \mathrm{m} \\
{ }^{2} \mathrm{D}: \text { DB-1701 } 1.5 \mathrm{~m} \times 0.1 \mathrm{~mm} \times 0.4 \mu \mathrm{m}\end{array}$ & LOD: $0.0021-0.048 \mu \mathrm{mol} / \mathrm{L}$ & [84] \\
\hline
\end{tabular}




\begin{tabular}{|c|c|c|c|c|c|}
\hline Matrix & Target analytes & Sample Preparation & Analysis & Comments & Reference \\
\hline & & & $\begin{array}{l}\text { Modulation: } 5 \mathrm{~s} \\
\text { Longitudinally modulated cryogenic system (LMCS) }\end{array}$ & & \\
\hline $\begin{array}{l}\text { Stenotrophom } \\
\text { onas } \\
\text { maltophilia } \\
\text { LMG 958T } \\
\text { bacteria } \\
\text { sample, algae }\end{array}$ & Fatty acids methyl ester & & $\begin{array}{l}\text { GC } \times \text { GC-qMS/FID } \\
{ }^{1} \text { D: HP-5MS } 30 \mathrm{~m} \times 0.25 \mathrm{~mm} \times 0.25 \mu \mathrm{m} \\
{ }^{2} \mathrm{D}: \text { BPX-70 } 4.0 \mathrm{~m} \times 0.25 \mathrm{~mm} \times 0.25 \mu \mathrm{m} \\
\text { Y-splitter to FID and qMS } \\
\text { Modulation : } 2 \mathrm{~s} \\
\text { Flow modulator }\end{array}$ & Profiling & {$[85-86]$} \\
\hline Soil & Phospholipid fatty acids & $\begin{array}{l}\text { Extraction: } \\
\text { chloroform- } \\
\text { methanoland citrate } \\
\text { buffer } \\
\text { Clean-up: SPE } \\
\text { Derivatization }\end{array}$ & $\begin{array}{l}\text { GC } \times \text { GC-qMS/FID and } \\
\text { Modulator: Everest model longitudinally modulated } \\
\text { cryogenic system } \\
{ }^{1} \mathrm{D} \text { : HP-5MS } 60 \mathrm{~m} \times 0.25 \mathrm{~mm} \times 0.25 \mu \mathrm{m} \\
{ }^{2} \mathrm{D}: \text { HP-INNOWax } 1.0 \mathrm{~m} \times 0.18 \mathrm{~mm} \times 0.18 \mu \mathrm{m} \\
\text { Modulation : } 6 \mathrm{~s} \\
{ }^{1} \mathrm{D}: \mathrm{HP}-8860 \mathrm{~m} \times 0.25 \mathrm{~mm} \times 0.25 \mu \mathrm{m} \\
{ }^{2} \mathrm{D}: \mathrm{HP}-\mathrm{INNOWax} 1.0 \mathrm{~m} \times 0.18 \mathrm{~mm} \times 0.18 \mu \mathrm{m} \\
\text { Modulation : } 6 \mathrm{~s} \\
{ }^{1} \mathrm{D}: \text { SolGel-Wax } 30 \mathrm{~m} \times 0.32 \mathrm{~mm} \times 0.32 \mu \mathrm{m} \\
{ }^{2} \mathrm{D}: \text { BPX-70 } 2.0 \mathrm{~m} \times 0.18 \mathrm{~mm} \times 0.18 \mu \mathrm{m} \\
\text { Modulation : } 6 \mathrm{~s}\end{array}$ & Profiling & {$[87-88]$} \\
\hline
\end{tabular}




\begin{tabular}{|c|c|c|c|c|c|}
\hline Matrix & Target analytes & Sample Preparation & Analysis & Comments & Reference \\
\hline & & & $\begin{array}{l}{ }^{1} \mathrm{D}: \mathrm{HP}-5 \mathrm{MS} 30 \mathrm{~m} \times 0.25 \mathrm{~mm} \times 0.25 \mu \mathrm{m} \\
{ }^{2} \mathrm{D}: \text { BPX-70 } 4.0 \mathrm{~m} \times 0.25 \mathrm{~mm} \times 0.25 \mu \mathrm{m} \\
\text { Modulation : } 5 \mathrm{~s} \\
\text { GC×GC-qTOF-MS } \\
{ }^{1} \mathrm{D}: \text { HP-5MS } 30 \mathrm{~m} \times 0.25 \mathrm{~mm} \times 0.25 \mu \mathrm{m} \\
{ }^{2} \mathrm{D}: \text { HP-INNOWax } 1.5 \mathrm{~m} \times 0.1 \mathrm{~mm} \times 0.1 \mu \mathrm{m} \\
\text { Modulation : } 10 \mathrm{~s}\end{array}$ & & \\
\hline Soil & Petroleum spill & & $\begin{array}{l}\text { GC } \times \text { GC-NCI-MS } \\
{ }^{1} \text { D: DB-5 } 25 \mathrm{~m} \times 0.25 \mathrm{~mm} \times 0.15 \mu \mathrm{m} \\
{ }^{2} \mathrm{D}: \text { Rxi-17SilMS } 1 \mathrm{~m} \times 0.1 \mathrm{~mm} \times 0.1 \mu \mathrm{m} \\
\text { Modulation: } 3 \mathrm{~s} \\
\text { Modulator: single-stage thermal modulator }\end{array}$ & $\begin{array}{l}\text { LOD: } 70 \text { pg for calibration } \\
\text { standards }\end{array}$ & [89] \\
\hline $\begin{array}{l}\text { Sediments and } \\
\text { fish }\end{array}$ & $\begin{array}{l}\text { SCCP } \\
\text { MCCP }\end{array}$ & & $\begin{array}{l}\text { GC } \times \text { GC-ECNI-HRTOF-MS } \\
{ }^{1} \text { D: DB- } 525 \mathrm{~m} \text { x } 0.25 \mathrm{~mm} \times 0.15 \mu \mathrm{m} \\
{ }^{2} \mathrm{D}: \text { BPX-50 } 1 \mathrm{~m} \text { x } 0.1 \mathrm{~mm} \times 0.1 \mu \mathrm{m} \\
\text { Modulation: } 8 \mathrm{~s}\end{array}$ & $\begin{array}{l}\text { MDL: } \\
\text { Sediments } 3.7 \text { and } 2.1 \mathrm{ng} / \mathrm{g} \text { for } \\
\text { the SCCPs and MCCP } \\
\text { Fish: } 9.4 \text { and } 7.0 \mathrm{ng} / \mathrm{g} \text { for the } \\
\text { SCCPs and MCCPs }\end{array}$ & [91-92] \\
\hline Sediments & SCCP & $\begin{array}{l}\text { Extraction: ASE } \\
\text { Clean-up: SPE }\end{array}$ & $\begin{array}{l}\mathrm{GC} \times \mathrm{GC}-\mu \mathrm{ECD} \\
{ }^{1} \mathrm{D}: \mathrm{DB}-130 \mathrm{~m} \times 0.25 \mathrm{~mm} \times 0.25 \mu \mathrm{m} \\
{ }^{2} \text { D: Rtx-PCB } 1.6 \mathrm{~m} \times 0.1 \mathrm{~mm} \times 0.1 \mu \mathrm{m} \\
\text { Modulation: } 4 \mathrm{~s}\end{array}$ & Recoveries: $120-130 \%$ & [93] \\
\hline Sediments & $\begin{array}{l}400 \text { compounds were tentatively } \\
\text { identified from } \\
\text { three fractions }\end{array}$ & & $\begin{array}{l}\text { GC } \times \text { GC-TOFMS } \\
{ }^{1} \text { D: DB-5 } 30 \mathrm{~m} \times 0.25 \mathrm{~mm} \times 0.25 \mu \mathrm{m}\end{array}$ & Screening & [94] \\
\hline
\end{tabular}




\begin{tabular}{|c|c|c|c|c|c|}
\hline Matrix & Target analytes & Sample Preparation & Analysis & Comments & Reference \\
\hline & & & $\begin{array}{l}{ }^{2} \mathrm{D}: 007-65 \mathrm{HT} \\
\text { Modulation: } 5 \mathrm{~s} \text { (valves modulator, } \mathrm{CO}_{2} \text { dual-jets) }\end{array}$ & & \\
\hline Dust & $\begin{array}{l}\text { PAHs, phthalates, halogen } \\
\text { containing compounds and nitro } \\
\text { compounds }\end{array}$ & $\begin{array}{l}\text { Extraction: ASE } \\
\text { Clean-up: GPC }\end{array}$ & $\begin{array}{l}\text { GC } \times \text { GC-TOFMS } \\
{ }^{1} \mathrm{D}: \text { Rxi5-MS } 18 \mathrm{~m} \text { x } 0.18 \mathrm{~mm} \times 0.18 \mu \mathrm{m} \\
{ }^{2} \mathrm{D}: \text { BPx-50 } 1.2 \mathrm{~m} \text { x } 0.1 \mathrm{~mm} \times 0.1 \mu \mathrm{m} \\
\text { Modulation: } 4 \mathrm{~s}\end{array}$ & $\begin{array}{l}\text { Screening } \\
\text { ChromaTOF scripts }\end{array}$ & [95] \\
\hline Sediments & $\begin{array}{l}\text { Dechloranes, } \mathrm{Cl}-, \mathrm{Br}-\text { and } \mathrm{Cl}-/ \mathrm{Br}- \\
\text { carbazoles, } \mathrm{POPs}\end{array}$ & $\begin{array}{l}\text { Extraction: Soxhlet } \\
\text { Clean-up: multi-layer } \\
\text { silica } \\
\text { Copper treatment }\end{array}$ & $\begin{array}{l}\text { GC } \times \text { GC-TOFMS } \\
{ }^{1} \mathrm{D}: \text { Rtx-5MS } 30 \mathrm{~m} \text { x } 0.25 \mathrm{~mm} \times 0.25 \mu \mathrm{m} \\
{ }^{2} \mathrm{D}: \text { BPx-50 } 1.6 \mathrm{~m} \times 0.15 \mathrm{~mm} \times 0.15 \mu \mathrm{m} \\
\text { Modulation: } 6 \mathrm{~s}\end{array}$ & $\begin{array}{l}\text { Screening } \\
\text { ChromaTOF scripts }\end{array}$ & [96] \\
\hline Soil & $\begin{array}{l}\text { Non-targeted } \\
\text { Targeted - } 50 \text { compounds; } \\
\text { PAHs, phthalic acid esthers, } \\
\text { phenolic compounds, hydrocarbons, } \\
\text { benzene derivatives and aliphatic } \\
\text { acids }\end{array}$ & $\begin{array}{l}\text { ASE } \\
\text { Clean-up: silica } \\
\text { column }\end{array}$ & $\begin{array}{l}\text { GC } \times \text { GC-TOFMS } \\
{ }^{1} \text { D: Rtx-5 } 30 \mathrm{~m} \text { x } 0.25 \mathrm{~mm} \times 0.25 \mu \mathrm{m} \\
{ }^{2} \mathrm{D}: \text { Rxi-17MS } 1.0 \mathrm{~m} \text { x } 0.1 \mathrm{~mm} \times 0.1 \mu \mathrm{m} \\
\text { Modulation: } 3 \mathrm{~s}\end{array}$ & $\begin{array}{l}\text { LODs: } 0.03-1.02 \mathrm{ng} / \mathrm{g} \\
\text { LOQ: } 0.1-3.0 \mathrm{ng} / \mathrm{g}\end{array}$ & [97] \\
\hline $\begin{array}{l}\text { Sewage } \\
\text { sludge }\end{array}$ & $\begin{array}{l}\text { Non-targeted } \\
\text { Targeted - } 99 \text { compounds: } \\
\text { PCBs, PAHs, Cl/Br-diphenyl } \\
\text { ethers, fragrances, other non-polar } \\
\text { compounds }\end{array}$ & $\begin{array}{l}\text { Extraction: PLE and } \\
\text { SPLE } \\
\text { Clean-up: GPC }\end{array}$ & $\begin{array}{l}\text { GC } \times \text { GC-HRTOF-MS } \\
{ }^{1} \mathrm{D}: \text { DB-5MS } 30 \mathrm{~m} \times 0.25 \mathrm{~mm} \times 0.25 \mu \mathrm{m} \\
{ }^{2} \mathrm{D}: \text { Rxi-17Sil } 1.1 \mathrm{~m} \times 0.25 \mathrm{~mm} \times 0.25 \mu \mathrm{m} \\
\text { Modulation: } 4 \mathrm{~s}\end{array}$ & LODs: $0.02-76 \mathrm{ng} / \mathrm{g}$ & [98] \\
\hline $\begin{array}{l}\text { Soil, } \\
\text { sediment, air } \\
\text { particulate }\end{array}$ & $\begin{array}{l}97 \text { PAHs: parent PAHs, } \\
\text { alkyl-PAHs, nitro-PAHs, } \\
\text { oxy-PAHs, thio- }\end{array}$ & & $\begin{array}{l}\text { GC } \times \text { GC-TOFMS } \\
{ }^{1} \mathrm{D}: \text { Rtx-5MS } 35 \mathrm{~m} \text { x } 0.25 \mathrm{~mm} \times 0.25 \mu \mathrm{m} \\
{ }^{2} \mathrm{D}: \text { Rxi-17 } 1.2 \mathrm{~m} \text { x } 0.1 \mathrm{~mm} \times 0.1 \mu \mathrm{m}\end{array}$ & & [106] \\
\hline
\end{tabular}




\begin{tabular}{|c|c|c|c|c|c|}
\hline Matrix & Target analytes & Sample Preparation & Analysis & Comments & Reference \\
\hline & $\begin{array}{l}\text { PAHs, high molecular weight PAHs } \\
\text { (MW } \geq 300 \text { ), bromo-PAHs, and } \\
\text { chloro-PAHs }\end{array}$ & & 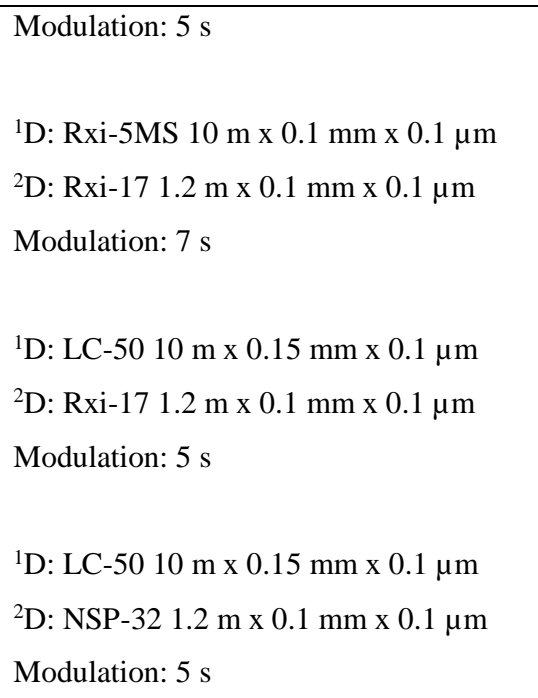 & & \\
\hline SRMs & $\begin{array}{l}\text { PAH mixtures in NIST SRMs: } \\
1650 \mathrm{~b} \text { and } 1975 \\
85 \text { different PAHs: parent, alkyl-, } \\
\text { nitro-, oxy-, thio-, bromo- and } \\
\text { chloro-PAHs }\end{array}$ & $\begin{array}{l}\text { NIST SRM1650b: } \\
\text { Extraction: PLE } \\
\text { Clean-up: } 20 \mathrm{~g} \text { silica } \\
\text { gel columns } \\
\text { NIST SRM1975: } \\
\text { Extraction: PLE } \\
\text { Clean-up: silica } \\
\text { No-clean-up }\end{array}$ & 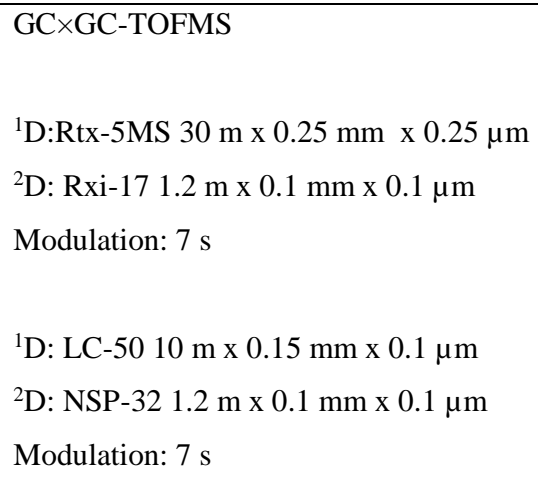 & $\begin{array}{l}\text { LOQs: } 3 \mathrm{pg} / \mathrm{L} \text { for PPAH to } 18 \\
\mathrm{pg} / \mathrm{L} \text { for } \mathrm{NPAH}\end{array}$ & [108] \\
\hline Soil, ash & Organic aromatic compounds & Extraction: ASE & $\begin{array}{l}\text { GC } \times \text { GC-TOFMS } \\
{ }^{1} \text { D: HT- } 830 \mathrm{~m} \text { x } 0.25 \mathrm{~mm} \times 0.25 \mu \mathrm{m} \\
{ }^{2} \mathrm{D}: \text { BPX- } 50 \text { or } 1.7 \mathrm{~m} \times 0.1 \mathrm{~mm} \times 0.1 \mu \mathrm{m} \\
\text { Modulation: } 6 \mathrm{~s}\end{array}$ & Qualitative & [155] \\
\hline
\end{tabular}




\begin{tabular}{|c|c|c|c|c|c|}
\hline Matrix & Target analytes & Sample Preparation & Analysis & Comments & Reference \\
\hline Soil & $\begin{array}{l}\text { PAH } \\
\text { Weathering of pollutants }\end{array}$ & & $\begin{array}{l}\text { GC } \times \text { GC-MS } \\
{ }^{1} \text { D: Rxi-5SilMS } 30 \mathrm{~m} \times 0.25 \mathrm{~mm} \times 0.25 \mu \mathrm{m} \\
{ }^{2} \mathrm{D} \text { : Rxi-17Sil } 1.1 \mathrm{~m} \times 0.25 \mathrm{~mm} \times 0.25 \mu \mathrm{m} \\
\text { Modulation: } 12 \mathrm{~s} \\
{ }^{1} \mathrm{D} \text { : DB-5 UI } 30 \mathrm{~m} \times 0.25 \mathrm{~mm} \times 1.0 \mu \mathrm{m} \\
{ }^{2} \mathrm{D} \text { : Rxi-17Sil } 1.5 \mathrm{~m} \times 0.18 \mathrm{~mm} \times 0.36 \mu \mathrm{m} \\
\text { Modulation: } 12 \mathrm{~s}\end{array}$ & Environmental forensics & [156] \\
\hline
\end{tabular}

Table A.4. - Recent applications of GC $\times$ GC for the analysis of air samples

\begin{tabular}{|c|c|c|c|c|c|}
\hline Matrix & Target analytes & Sample Preparation & Analysis & Comments / Method Performance & Reference \\
\hline $\begin{array}{l}\text { Athmospheric } \\
\text { particle } \\
\text { SRM 1649a } \\
\text { urban dust } \\
\text { SRM 1650b } \\
\text { diesel exhaust } \\
\text { particles }\end{array}$ & $\begin{array}{l}29 \text { compounds: } 11 \text { PAHs, } 9 \text { oxy- } \\
\text { PAHs, } 6 \text { nitro-PAHs, and } 3 \text { methyl- } \\
\text { PAHs }\end{array}$ & & $\begin{array}{l}\text { TD-GCxGC-MS/MS } \\
{ }^{1} \text { D: InertCap 5MS/Sil } 60 \mathrm{~m} \times 0.25 \mathrm{~mm} \times 0.1 \mu \mathrm{m} \\
{ }^{2} \mathrm{D}: \text { BPX-50 } 1.5 \mathrm{~m} \times 0.1 \mathrm{~mm} \times 0.1 \mu \mathrm{m} \\
\text { Modulation: } 5 \mathrm{~s}\end{array}$ & $\begin{array}{l}\text { IDLs: } \\
\text { PAHs } 0.03-0.3 \mathrm{pg} \\
\text { Oxygenated PAHs } 0.04-0.2 \mathrm{pg} \\
\text { Nitrated PAHs } 0.03-0.1 \mathrm{pg} \\
\text { Methylated PAHs } 0.01-0.08 \mathrm{pg}\end{array}$ & [103-105] \\
\hline $\begin{array}{l}\text { Particulate } \\
\text { matter, diesel }\end{array}$ & $\begin{array}{l}\text { PAHs, alkyl-PAHs, nitro-PAHs, } \\
\text { oxy-PAHs, thio-PAHs, high } \\
\text { molecular weight PAHs (MW } \geq\end{array}$ & $\begin{array}{l}\text { Extraction: PLE } \\
\text { Clean-up: silica }\end{array}$ & $\begin{array}{l}\text { GCxGC-TOF-MS } \\
\text { Rtx-5MS } \times \text { Rxi-17 }\end{array}$ & Quantitative & [106-107] \\
\hline
\end{tabular}




\begin{tabular}{|c|c|c|c|c|c|}
\hline Matrix & Target analytes & Sample Preparation & Analysis & Comments / Method Performance & Reference \\
\hline $\begin{array}{l}\text { particulate } \\
\text { extract }\end{array}$ & $\begin{array}{l}\text { 300), bromo-PAHs, and chloro- } \\
\text { PAHs }\end{array}$ & & $\begin{array}{l}\text { Rxi-5MS×Rxi-17 } \\
\text { LC-50×Rxi-17 } \\
\text { LC-50×NSP-35 } \\
\text { Modulation: } 5 \mathrm{~s}\end{array}$ & & \\
\hline Air samples & VOCs & $\begin{array}{l}\text { Thermal desorption } \\
\text { tubes used to collect } \\
\text { samples }\end{array}$ & $\begin{array}{l}\text { TD-GCxGC-qMS and GCxGC-FID } \\
{ }^{1} \mathrm{D}: \text { HP-5MS } 30 \mathrm{~m} \text { x } 0.25 \mathrm{~mm} \times 0.25 \mu \mathrm{m} \\
{ }^{2} \mathrm{D}: \text { HP-INNOWAX } 2.5 \mathrm{~m} \times 0.18 \mathrm{~mm} \times 0.18 \mu \mathrm{m} \\
\text { Modulation: } 6 \mathrm{~s}\end{array}$ & qualitative & {$[108]$} \\
\hline Air samples & VOCs & $\begin{array}{l}\text { Carbopack adsorbtion } \\
\text { followed by thermal } \\
\text { desorption }\end{array}$ & $\begin{array}{l}\text { Miniaturized GC×GC- } \mu \mathrm{PID} \\
{ }^{1} \mathrm{D}: \text { Rtx-5MS } 10 \mathrm{~m} \text { x } 0.25 \mathrm{~mm} \times 0.25 \mu \mathrm{m} \\
{ }^{2} \mathrm{D}: \text { Rtx-200 } 3 \mathrm{~m} \text { x } 0.25 \mathrm{~mm} \text { x } 0.25 \mu \mathrm{m} \\
\text { Modulation: } 8 \mathrm{~s}\end{array}$ & quantitative & [109] \\
\hline
\end{tabular}




\begin{tabular}{|c|c|c|c|c|c|}
\hline Matrix & Target analytes & Sample Preparation & Analysis & Comments / Method Performance & Reference \\
\hline Aerosol & Organic compounds & $\begin{array}{l}\text { Impactor particle } \\
\text { collector - thermal } \\
\text { desorption }\end{array}$ & $\begin{array}{l}\text { GC } \times \text { GC-FID } \\
{ }^{1} \mathrm{D}: \text { VF-5MS } 30 \mathrm{~m} \times 0.25 \mathrm{~mm} \times 0.25 \mu \mathrm{m} \\
{ }^{2} \mathrm{D} \text { : Solgel Wax } 1.4 \mathrm{~m} \times 0.25 \mathrm{~mm} \times 0.25 \mu \mathrm{m} \\
\text { Modulation: } 6 \mathrm{~s} \\
\text { Thermal modulator: custom build }\end{array}$ & Qualitative & [119] \\
\hline
\end{tabular}

References

[149] G. Semard, A. Bruchet, P. Cardinaël , J. Bouillon, Use of comprehensive two- dimensional gas chromatography for the broad screening of hazardous contaminants in urban wastewaters, Water Sci. Technol. 57 (2008) 1983-1989.

[150] S. Herrera López, M.M. Ulaszewska, M.D. Hernando, M.J. Martínez Bueno, M.J. Gómez, A.R. Fernández-Alba, Post-acquisition data processing for the screening of transformation products of different organic contaminants. Two-year monitoring of river water using LC-ESI-QTOF-MS and GCxGC-EI-TOF-MS, Environ. Sci. Pollut. R. 21 (2014) 12583-12604.

[151] Q. Guo, X. Li, J. Yu, H. Zhang, Y. Zhang, M. Yang, N. Lu, D. Zhang, Comprehensive two- dimensional gas chromatography with time-of-flight mass spectrometry for the screening of potent swampy/septic odor-causing compounds in two drinking water sources in China, Anal. Methods 7 (2015) $2458-2468$.

[152] M Beldean-Galea, D. Thébaut, J. Vial, V. Coman, Identification of complex volatile organic compounds in municipal landfill leachate by head-space solid phase microextraction and GCxGC-qMS analysis, Studia UBB Chemia 62 (2017) 35-44.

[153] W. Yang, Z.Q. Yu, L. Xiang-Fan, F. Jia-Liang, Z. Dong-Ping, R. Guo-Fa, S. Guo-Ying, F. Jia-Mo, Qualitative Analysis of Some Emerging Halogenous Pollutions in Fish Sample by Comprehensive Two- Dimensional Gas Chromatography/Time-of-Flight Mass Spectrometry, Chinese J. Anal. Chem. 40 (2012) $1187-1193$. 
[154] A. Goto, N.M. Tue, M. Someya, T. Isobe, S. Takahashi, S. Tanabe, T. Kunisue, Occurrence of natural mixed halogenated dibenzo-p-dioxins: Specific distribution and profiles in mussels from Seto Inland Sea, Japan, Environ. Sci. Technol. 51 (2017) 11771.

[155] J. Escobar-Arnanz, S. Mekni, G. Blanco, E. Eljarrat, D. Barceló, L. Ramos, Characterization of organic aromatic compounds in soils affected by an uncontrolled tire landfill fire through the use of comprehensive two- dimensional gas chromatography-time-of-flight mass spectrometry, J. Chromatogr. A 1536 (2018) $163-175$.

[156] P. M._Antle, C. D. Zeigler, D. G. Livitz, A. Robbat, Two-dimensional gas chromatography/mass spectrometry, physical property modeling and automated production of component maps to assess the weathering of pollutants, J. Chromatogr. A 1364 (2014) 223-233.

[157] E. Hoh, S.J. Lehotay, K. Mastovska, J.K. Huwe, Evaluation of automated direct sample introduction with comprehensive two- dimensional gas chromatography/time-offlight mass spectrometry for the screening analysis of dioxins in fish oil, J. Chromatogr. A 1201 (2008) 69-77. 


\section{Appendix B}

\section{Evaluation of a Single-Stage Consumable-Free Modulator for GCXGC: Analysis of Polychlorinated Biphenyls, Organochlorine Pesticides and Chlorobenzenes}

Table B.1 Repeatability - Full Width at Half Height (FWHH)

\begin{tabular}{|c|c|c|c|c|c|c|c|c|}
\hline \multirow{2}{*}{$\begin{array}{c}\mathrm{N}=10 \text { Replicates } \\
\text { Name }\end{array}$} & \multicolumn{4}{|c|}{$\mathbf{L N}_{2}$ Modulator } & \multicolumn{4}{|c|}{ University of Waterloo Modulator } \\
\hline & $\begin{array}{c}{ }^{1} \mathbf{D} \\
\text { Retention } \\
\text { Time (s) }\end{array}$ & $\begin{array}{c}{ }^{2} \mathrm{D} \\
\text { Retention } \\
\text { Time (s) }\end{array}$ & $\begin{array}{l}\text { FWHH } \\
\text { Avg. } \\
\text { (s) }\end{array}$ & $\begin{array}{c}\text { FWHH } \\
\text { Std. } \\
\text { Deviation } \\
\text { (s) }\end{array}$ & $\begin{array}{c}{ }^{1} \mathbf{D} \\
\text { Retention } \\
\text { Time (s) }\end{array}$ & $\begin{array}{c}{ }^{2} \mathrm{D} \\
\text { Retention } \\
\text { Time (s) }\end{array}$ & $\begin{array}{c}\text { FWHH } \\
\text { Avg. } \\
\text { (s) }\end{array}$ & $\begin{array}{l}\text { FWHH } \\
\text { Std. } \\
\text { Deviation } \\
(\mathbf{s}) \\
\end{array}$ \\
\hline PCB4/PCB10 & 884 & 0.240 & 0.151 & 0.001 & 879 & 0.124 & 0.192 & 0.004 \\
\hline PCB6 & 960 & 0.524 & 0.164 & 0.001 & 952 & 0.568 & 0.207 & 0.003 \\
\hline PCB8 & 972 & 0.660 & 0.169 & 0.001 & 964 & 0.776 & 0.215 & 0.004 \\
\hline PCB15 & 1072 & 1.346 & 0.189 & 0.001 & 1061 & 1.854 & 0.245 & 0.003 \\
\hline PCB16 & 1108 & 1.014 & 0.174 & 0.001 & 1171 & 1.450 & 0.233 & 0.020 \\
\hline PCB18 & 1064 & 0.844 & 0.172 & 0.001 & 1058 & 1.028 & 0.223 & 0.005 \\
\hline PCB19 & 1012 & 0.718 & 0.168 & 0.001 & 1205 & 1.308 & 0.236 & 0.031 \\
\hline PCB22 & 1212 & 1.506 & 0.183 & 0.001 & 1202 & 2.004 & 0.240 & 0.003 \\
\hline PCB28/PCB31 & 1176 & 1.384 & 0.189 & 0.001 & 1164 & 1.870 & 0.268 & 0.013 \\
\hline PCB33 & 1196 & 1.310 & 0.182 & 0.002 & 1188 & 1.682 & 0.237 & 0.003 \\
\hline PCB37 & 1312 & 2.046 & 0.211 & 0.000 & 1342 & 2.156 & 0.250 & 0.004 \\
\hline PCB40 & 1352 & 1.678 & 0.188 & 0.001 & 1342 & 2.156 & 0.250 & 0.004 \\
\hline PCB41 & 1336 & 1.602 & 0.185 & 0.001 & 1326 & 2.048 & 0.245 & 0.003 \\
\hline PCB44 & 1304 & 1.522 & 0.187 & 0.001 & 1297 & 1.952 & 0.258 & 0.012 \\
\hline PCB49 & 1272 & 1.384 & 0.186 & 0.001 & 1261 & 1.850 & 0.245 & 0.004 \\
\hline PCB52 & 1260 & 1.344 & 0.182 & 0.001 & 1250 & 1.816 & 0.246 & 0.005 \\
\hline PCB54 & 1136 & 1.108 & 0.178 & 0.001 & 1129 & 1.324 & 0.235 & 0.006 \\
\hline РCB66 & 1412 & 1.926 & 0.209 & 0.002 & 1402 & 2.604 & 0.273 & 0.011 \\
\hline PCB70 & 1407 & 1.857 & 0.182 & 0.005 & 1394 & 2.538 & 0.279 & 0.007 \\
\hline PCB74* & $\mathrm{n} / \mathrm{a}$ & $\mathrm{n} / \mathrm{a}$ & $\mathrm{n} / \mathrm{a}$ & $\mathrm{n} / \mathrm{a}$ & 1383 & 2.572 & 0.249 & 0.022 \\
\hline PCB77 & 1560 & 2.536 & 0.219 & 0.001 & 1548 & 3.460 & 0.300 & 0.004 \\
\hline PCB81 & 1536 & 2.434 & 0.214 & 0.016 & 1526 & 3.288 & 0.295 & 0.004 \\
\hline PCB84 & 1464 & 1.872 & 0.198 & 0.006 & 1455 & 2.398 & 0.266 & 0.004 \\
\hline PCB85 & 1548 & 2.086 & 0.218 & 0.009 & 1538 & 2.770 & 0.284 & 0.017 \\
\hline PCB87 & 1536 & 2.028 & 0.199 & 0.006 & 1528 & 2.638 & 0.265 & 0.005 \\
\hline PCB90/PCB101 & 1479 & 1.712 & 0.191 & 0.003 & 1467 & 2.306 & 0.254 & 0.004 \\
\hline PCB95 & 1416 & 1.664 & 0.234 & 0.018 & 1406 & 2.186 & 0.267 & 0.022 \\
\hline
\end{tabular}


Table B.1 Repeatability - Full Width at Half Height (FWHH)

\begin{tabular}{|c|c|c|c|c|c|c|c|c|}
\hline \multirow{2}{*}{$\begin{array}{c}\mathbf{N = 1 0} \text { Replicates } \\
\text { Name }\end{array}$} & \multicolumn{4}{|c|}{$\mathbf{L N}_{2}$ Modulator } & \multicolumn{4}{|c|}{ University of Waterloo Modulator } \\
\hline & $\begin{array}{c}{ }^{1} \mathbf{D} \\
\text { Retention } \\
\text { Time (s) }\end{array}$ & $\begin{array}{c}{ }^{2} \mathbf{D} \\
\text { Retention } \\
\text { Time (s) }\end{array}$ & $\begin{array}{l}\text { FWHH } \\
\text { Avg. } \\
\text { (s) }\end{array}$ & $\begin{array}{c}\text { FWHH } \\
\text { Std. } \\
\text { Deviation } \\
(\mathbf{s}) \\
\end{array}$ & $\begin{array}{c}{ }^{1} \mathbf{D} \\
\text { Retention } \\
\text { Time (s) }\end{array}$ & $\begin{array}{c}{ }^{2} \mathrm{D} \\
\text { Retention } \\
\text { Time (s) }\end{array}$ & $\begin{array}{c}\text { FWHH } \\
\text { Avg. } \\
\text { (s) }\end{array}$ & $\begin{array}{c}\text { FWHH } \\
\text { Std. } \\
\text { Deviation } \\
(\mathbf{s}) \\
\end{array}$ \\
\hline PCB97 & 1528 & 1.844 & 0.200 & 0.015 & 1518 & 2.394 & 0.260 & 0.004 \\
\hline PCB99** & 1488 & 1.790 & 0.205 & 0.004 & 1485 & 2.354 & 0.258 & 0.010 \\
\hline PCB104 & 1300 & 1.310 & 0.182 & 0.001 & 1291 & 1.638 & 0.246 & 0.004 \\
\hline PCB105 & 1688 & 2.578 & 0.210 & 0.000 & 1678 & 3.360 & 0.281 & 0.003 \\
\hline PCB110 & 1564 & 2.044 & 0.198 & 0.000 & 1553 & 2.676 & 0.266 & 0.006 \\
\hline PCB114 & $\mathrm{n} / \mathrm{a}$ & $\mathrm{n} / \mathrm{a}$ & $\mathrm{n} / \mathrm{a}$ & $\mathrm{n} / \mathrm{a}$ & 1646 & 3.124 & 0.322 & 0.028 \\
\hline PCB118 & 1632 & 2.276 & 0.206 & 0.001 & 1620 & 3.066 & 0.288 & 0.009 \\
\hline PCB119 & 1508 & 1.822 & 0.192 & 0.001 & 1498 & 2.392 & 0.258 & 0.004 \\
\hline PCB123 & 1624 & 2.204 & 0.202 & 0.001 & 1614 & 2.904 & 0.275 & 0.005 \\
\hline PCB126 & 1776 & 2.828 & 0.222 & 0.001 & 1764 & 3.782 & 0.308 & 0.005 \\
\hline PCB128 & 1816 & 2.620 & 0.211 & 0.000 & 1806 & 3.324 & 0.285 & 0.004 \\
\hline PCB129 & 1776 & 2.252 & 0.201 & 0.001 & 1765 & 2.812 & 0.270 & 0.005 \\
\hline PCB135 & 1612 & 1.842 & 0.196 & 0.000 & 1601 & 2.344 & 0.265 & 0.009 \\
\hline PCB137 & 1739 & 2.184 & 0.219 & 0.017 & 1727 & 2.796 & 0.273 & 0.004 \\
\hline PCB138 & 1756 & 2.306 & 0.203 & 0.001 & 1746 & 2.936 & 0.276 & 0.004 \\
\hline PCB141 & 1724 & 2.128 & 0.197 & 0.001 & 1714 & 2.710 & 0.266 & 0.003 \\
\hline РCB149 & 1628 & 1.906 & 0.212 & 0.003 & 1619 & 2.440 & 0.267 & 0.003 \\
\hline PCB151 & 1603 & 1.848 & 0.196 & 0.001 & 1593 & 2.372 & 0.326 & 0.072 \\
\hline PCB153 & 1696 & 2.012 & 0.199 & 0.001 & 1661 & 2.546 & 0.269 & 0.009 \\
\hline PCB155 & 1460 & 1.452 & 0.184 & 0.001 & 1493 & 2.002 & 0.256 & 0.017 \\
\hline PCB156 & 1884 & 2.728 & 0.224 & 0.001 & 1872 & 3.560 & 0.307 & 0.008 \\
\hline PCB157 & 1892 & 2.774 & 0.216 & 0.001 & 1869 & 3.496 & 0.291 & 0.006 \\
\hline PCB158 & 1764 & 2.342 & 0.204 & 0.000 & 1689 & 2.832 & 0.270 & 0.014 \\
\hline PCB167**** & 1832 & 2.500 & 0.210 & 0.001 & $\mathrm{n} / \mathrm{a}$ & $\mathrm{n} / \mathrm{a}$ & $\mathrm{n} / \mathrm{a}$ & $\mathrm{n} / \mathrm{a}$ \\
\hline PCB168 & 1704 & 1.932 & 0.195 & 0.001 & 1693 & 2.484 & 0.265 & 0.005 \\
\hline PCB169 & 1980 & 3.054 & 0.225 & 0.001 & 1993 & 3.718 & 0.302 & 0.005 \\
\hline PCB170 & 1996 & 2.676 & 0.213 & 0.001 & 1985 & 3.322 & 0.289 & 0.004 \\
\hline PCB174 & 1860 & 2.206 & 0.200 & 0.000 & 1850 & 2.708 & 0.270 & 0.004 \\
\hline PCB177 & 1872 & 2.382 & 0.207 & 0.001 & 1862 & 2.966 & 0.278 & 0.004 \\
\hline PCB178 & 1788 & 1.990 & 0.197 & 0.001 & 1778 & 2.478 & 0.267 & 0.004 \\
\hline PCB180 & 1932 & 2.390 & 0.205 & 0.001 & 1911 & 2.978 & 0.276 & 0.005 \\
\hline PCB183 & 1820 & 2.088 & 0.198 & 0.001 & 1821 & 2.622 & 0.268 & 0.003 \\
\hline PCB187 & 1808 & 2.038 & 0.198 & 0.001 & 1786 & 2.518 & 0.270 & 0.005 \\
\hline PCB188 & 1680 & 1.784 & 0.201 & 0.001 & 1722 & 2.386 & 0.268 & 0.015 \\
\hline PCB189 & 2072 & 2.918 & 0.217 & 0.001 & 1916 & 3.292 & 0.281 & 0.034 \\
\hline PCB191 & 1952 & 2.386 & 0.206 & 0.001 & 1845 & 2.798 & 0.270 & 0.020 \\
\hline
\end{tabular}


Table B.1 Repeatability - Full Width at Half Height (FWHH)

\begin{tabular}{|c|c|c|c|c|c|c|c|c|}
\hline \multirow{2}{*}{$\begin{array}{c}\mathbf{N = 1 0} \text { Replicates } \\
\text { Name }\end{array}$} & \multicolumn{4}{|c|}{$\mathbf{L N}_{2}$ Modulator } & \multicolumn{4}{|c|}{ University of Waterloo Modulator } \\
\hline & $\begin{array}{c}{ }^{1} \mathbf{D} \\
\text { Retention } \\
\text { Time (s) }\end{array}$ & $\begin{array}{c}{ }^{2} \mathbf{D} \\
\text { Retention } \\
\text { Time (s) }\end{array}$ & $\begin{array}{l}\text { FWHH } \\
\text { Avg. } \\
\text { (s) }\end{array}$ & $\begin{array}{l}\text { FWHH } \\
\text { Std. } \\
\text { Deviation } \\
\text { (s) }\end{array}$ & $\begin{array}{c}{ }^{1} \mathbf{D} \\
\text { Retention } \\
\text { Time (s) }\end{array}$ & $\begin{array}{c}{ }^{2} \mathbf{D} \\
\text { Retention } \\
\text { Time (s) }\end{array}$ & $\begin{array}{c}\text { FWHH } \\
\text { Avg. } \\
\text { (s) }\end{array}$ & $\begin{array}{c}\text { FWHH } \\
\text { Std. } \\
\text { Deviation } \\
\text { (s) }\end{array}$ \\
\hline PCB193 & 1940 & 2.360 & 0.204 & 0.000 & 1931 & 2.956 & 0.277 & 0.004 \\
\hline PCB194 & 2160 & 2.718 & 0.213 & 0.001 & 2149 & 3.360 & 0.293 & 0.005 \\
\hline PCB199 & 2028 & 2.314 & 0.204 & 0.001 & 2020 & 2.782 & 0.275 & 0.002 \\
\hline PCB200 & 1960 & 2.336 & 0.203 & 0.001 & 1952 & 2.750 & 0.273 & 0.006 \\
\hline PCB201 & 1908 & 2.110 & 0.200 & 0.000 & 1900 & 2.512 & 0.269 & 0.005 \\
\hline РCB202 & 1888 & 2.064 & 0.202 & 0.001 & 1881 & 2.450 & 0.272 & 0.009 \\
\hline PCB203 & 2044 & 2.378 & 0.203 & 0.001 & 2032 & 2.898 & 0.278 & 0.005 \\
\hline PCB205 & 2176 & 2.758 & 0.213 & 0.001 & 2166 & 3.398 & 0.292 & 0.002 \\
\hline РCB206 & 2254 & 2.624 & 0.218 & 0.007 & 2245 & 3.152 & 0.288 & 0.005 \\
\hline РCB207 & 2132 & 2.394 & 0.204 & 0.001 & 2124 & 2.778 & 0.274 & 0.003 \\
\hline PCB208 & 2116 & 2.300 & 0.202 & 0.001 & 2106 & 2.676 & 0.273 & 0.006 \\
\hline PCB209 & 2328 & 2.596 & 0.210 & 0.000 & 2322 & 2.942 & 0.288 & 0.006 \\
\hline HCB & 996 & 0.854 & 0.163 & 0.001 & 989 & 0.938 & 0.204 & 0.005 \\
\hline HCBD & 536 & 2.744 & 0.117 & 0.001 & $\mathrm{n} / \mathrm{a}$ & $\mathrm{n} / \mathrm{a}$ & $\mathrm{n} / \mathrm{a}$ & $\mathrm{n} / \mathrm{a}$ \\
\hline HCE & 417 & 2.768 & 0.118 & 0.002 & $\mathrm{n} / \mathrm{a}$ & $\mathrm{n} / \mathrm{a}$ & $\mathrm{n} / \mathrm{a}$ & $\mathrm{n} / \mathrm{a}$ \\
\hline $1,2,3,4-$ TCB & 656 & 3.426 & 0.131 & 0.000 & 654 & 2.920 & 0.155 & 0.005 \\
\hline $\begin{array}{c}1,2,3,5-/ 1,2,4,5- \\
\text { TCB }\end{array}$ & 620 & 3.206 & 0.129 & 0.001 & 616 & 2.606 & 0.158 & 0.004 \\
\hline 1,2,3-ТCB & 522 & 3.038 & 0.127 & 0.001 & 520 & 2.452 & 0.153 & 0.017 \\
\hline 1,2,4-ТCB & 495 & 3.000 & 0.130 & 0.002 & 492 & 2.428 & 0.163 & 0.031 \\
\hline 1,3,5-ТВB & 696 & 3.650 & 0.136 & 0.002 & 692 & 3.214 & 0.159 & 0.004 \\
\hline 1,3,5-ТCB & 461 & 2.910 & 0.125 & 0.002 & 458 & 2.330 & 0.161 & 0.009 \\
\hline 2,3,6-TCT & 600 & 3.114 & 0.128 & 0.001 & 599 & 2.528 & 0.154 & 0.008 \\
\hline 2,4,5-TCT & 592 & 3.092 & 0.127 & 0.001 & 590 & 2.516 & 0.157 & 0.006 \\
\hline P5CB & 793 & 0.020 & 0.145 & 0.002 & 784 & 3.770 & 0.174 & 0.004 \\
\hline A2,6-TCT & 628 & 3.286 & 0.129 & 0.002 & 625 & 2.738 & 0.151 & 0.007 \\
\hline A-BHC & 957 & 0.950 & 0.163 & 0.001 & 950 & 1.166 & 0.210 & 0.005 \\
\hline G-BHC & 1024 & 1.300 & 0.174 & 0.001 & 1016 & 1.704 & 0.223 & 0.005 \\
\hline A-CHLA** & 1496 & 1.940 & 0.193 & 0.001 & 1485 & 2.354 & 0.258 & 0.010 \\
\hline Oxy-CHLA & 1408 & 1.624 & 0.213 & 0.012 & 1397 & 1.920 & 0.241 & 0.007 \\
\hline Aldrin & 1312 & 1.354 & 0.177 & 0.001 & 1302 & 1.480 & 0.224 & 0.003 \\
\hline Endrin & 1596 & 2.638 & 0.208 & 0.001 & 1586 & 3.142 & 0.270 & 0.002 \\
\hline Dieldrin & 1548 & 2.364 & 0.238 & 0.012 & 1541 & 2.820 & 0.295 & 0.040 \\
\hline Endos 1 & 1488 & 2.184 & 0.198 & 0.001 & 1477 & 2.630 & 0.286 & 0.025 \\
\hline Endos 2 & 1604 & 3.482 & 0.234 & 0.001 & 1699 & 0.856 & 0.334 & 0.003 \\
\hline Endos S & 1705 & 3.680 & 0.240 & 0.001 & 1699 & 0.856 & 0.334 & 0.003 \\
\hline
\end{tabular}


Table B.1 Repeatability - Full Width at Half Height (FWHH)

\begin{tabular}{|c|c|c|c|c|c|c|c|c|}
\hline N=10 Replicates & & $\mathbf{L N}_{2} \mathbf{M o}$ & lulator & & Unive & sity of Wat & rloo Mod & lator \\
\hline Name & $\begin{array}{c}{ }^{1} \mathbf{D} \\
\text { Retention } \\
\text { Time (s) }\end{array}$ & $\begin{array}{c}{ }^{2} \mathbf{D} \\
\text { Retention } \\
\text { Time (s) }\end{array}$ & $\begin{array}{c}\text { FWHH } \\
\text { Avg. } \\
\text { (s) }\end{array}$ & $\begin{array}{c}\text { FWHH } \\
\text { Std. } \\
\text { Deviation } \\
(\mathbf{s}) \\
\end{array}$ & $\begin{array}{c}{ }^{1} \mathrm{D} \\
\text { Retention } \\
\text { Time (s) }\end{array}$ & $\begin{array}{c}{ }^{2} \mathrm{D} \\
\text { Retention } \\
\text { Time (s) }\end{array}$ & $\begin{array}{c}\text { FWHH } \\
\text { Avg. } \\
\text { (s) }\end{array}$ & $\begin{array}{c}\text { FWHH } \\
\text { Std. } \\
\text { Deviation } \\
\text { (s) } \\
\end{array}$ \\
\hline Heptachlor & 1224 & 1.206 & 0.172 & 0.001 & 1214 & 1.370 & 0.224 & 0.004 \\
\hline OCSTYR & 1402 & 1.186 & 0.172 & 0.001 & 1393 & 1.372 & 0.220 & 0.004 \\
\hline Trans-Nonachlor & 1516 & 1.826 & 0.195 & 0.011 & 1506 & 2.226 & 0.255 & 0.003 \\
\hline o,p'-DDT & 1660 & 1.960 & 0.196 & 0.003 & 1651 & 2.452 & 0.289 & 0.007 \\
\hline p,p'-DDD & 1640 & 2.522 & 0.206 & 0.004 & 1628 & 3.520 & 0.290 & 0.007 \\
\hline p,p'-DDE & 1548 & 1.840 & 0.233 & 0.010 & 1537 & 2.510 & 0.284 & 0.018 \\
\hline p,p'-DDT & 1744 & 2.380 & 0.208 & 0.011 & 1733 & 3.128 & 0.285 & 0.013 \\
\hline Mirex & 2000 & 3.436 & 0.239 & 0.001 & 1971 & 0.046 & 0.317 & 0.004 \\
\hline p-Mirex $* * *$ & 1832 & 2.990 & 0.223 & 0.007 & $\mathrm{n} / \mathrm{a}$ & $\mathrm{n} / \mathrm{a}$ & $\mathrm{n} / \mathrm{a}$ & $\mathrm{n} / \mathrm{a}$ \\
\hline G-CHLA/PCB60* & 1452 & 2.182 & 0.204 & 0.001 & 1442 & 2.838 & 0.276 & 0.005 \\
\hline DMDT/PCB171 & 1880 & 2.416 & 0.270 & 0.003 & 1872 & 3.020 & 0.303 & 0.009 \\
\hline $\begin{array}{c}\text { Cis- } \\
\text { Nonachlor/PCB114 } \\
\end{array}$ & 1660 & 2.356 & 0.205 & 0.001 & $\mathrm{n} / \mathrm{a}$ & $\mathrm{n} / \mathrm{a}$ & $\mathrm{n} / \mathrm{a}$ & $\mathrm{n} / \mathrm{a}$ \\
\hline H-Epoxide/PCB74 & 1396 & 1.840 & 0.195 & 0.001 & $\mathrm{n} / \mathrm{a}$ & $\mathrm{n} / \mathrm{a}$ & $\mathrm{n} / \mathrm{a}$ & $\mathrm{n} / \mathrm{a}$ \\
\hline H-Epoxide & $\mathrm{n} / \mathrm{a}$ & $\mathrm{n} / \mathrm{a}$ & $\mathrm{n} / \mathrm{a}$ & $\mathrm{n} / \mathrm{a}$ & 1386 & 2.244 & 0.295 & 0.018 \\
\hline Cis-Nonachlor & $\mathrm{n} / \mathrm{a}$ & $\mathrm{n} / \mathrm{a}$ & $\mathrm{n} / \mathrm{a}$ & $\mathrm{n} / \mathrm{a}$ & 1650 & 2.894 & 0.295 & 0.026 \\
\hline $\begin{array}{l}\text { PCB99/Alpha- } \\
\text { Chlordane }\end{array}$ & $\mathrm{n} / \mathrm{a}$ & $\mathrm{n} / \mathrm{a}$ & $\mathrm{n} / \mathrm{a}$ & $\mathrm{n} / \mathrm{a}$ & 1485 & 2.354 & 0.258 & 0.010 \\
\hline $\begin{array}{l}\text { PCB167/Photo- } \\
\text { Mirex }\end{array}$ & $\mathrm{n} / \mathrm{a}$ & $\mathrm{n} / \mathrm{a}$ & $\mathrm{n} / \mathrm{a}$ & $\mathrm{n} / \mathrm{a}$ & 1810 & 3.242 & 0.293 & 0.011 \\
\hline \multicolumn{9}{|c|}{$\begin{array}{l}* \mathrm{PCB} 74 / \mathrm{H} \text {-epoxide coelutopn - } \mathrm{LN}_{2} \\
\text { modulator }\end{array}$} \\
\hline \multicolumn{9}{|c|}{$\begin{array}{l}\text { **PCB99/alpha-chlordane coelution - UW } \\
\text { modulator }\end{array}$} \\
\hline $\begin{array}{l}* * * \mathrm{PCB} 167 / \text { photo-M } \\
\text { modulator }\end{array}$ & rex coelutior & - UW & & & & & & \\
\hline
\end{tabular}


Table B.2 Repeatability- Peak Heights

\begin{tabular}{|c|c|c|c|c|}
\hline \multirow{2}{*}{$\begin{array}{c}\mathrm{N}=10 \text { Replicates } \\
\text { Name }\end{array}$} & \multicolumn{2}{|c|}{$\mathbf{L N}_{2}$ Modulator } & \multicolumn{2}{|c|}{$\begin{array}{c}\begin{array}{c}\text { University of Waterloo } \\
\text { Modulator }\end{array} \\
\end{array}$} \\
\hline & Avg. & $\begin{array}{c}\text { Std. } \\
\text { Deviation }\end{array}$ & Avg. & $\begin{array}{c}\text { Std. } \\
\text { Deviation }\end{array}$ \\
\hline PCB4/PCB10 & $7.28 \mathrm{E}+04$ & $1.87 \mathrm{E}+03$ & $8.29 \mathrm{E}+04$ & $5.17 \mathrm{E}+03$ \\
\hline PCB6 & $4.71 \mathrm{E}+04$ & $1.41 \mathrm{E}+03$ & $5.68 \mathrm{E}+04$ & $3.17 \mathrm{E}+03$ \\
\hline PCB8 & $5.43 \mathrm{E}+04$ & $1.60 \mathrm{E}+03$ & $6.42 \mathrm{E}+04$ & $3.51 \mathrm{E}+03$ \\
\hline PCB15 & $1.63 \mathrm{E}+04$ & $5.90 \mathrm{E}+02$ & $2.00 \mathrm{E}+04$ & $7.63 \mathrm{E}+02$ \\
\hline PCB16 & $8.21 \mathrm{E}+04$ & $1.55 \mathrm{E}+03$ & $1.37 \mathrm{E}+05$ & $1.38 \mathrm{E}+05$ \\
\hline PCB18 & $5.89 \mathrm{E}+04$ & $1.19 \mathrm{E}+03$ & $6.64 \mathrm{E}+04$ & $4.31 \mathrm{E}+03$ \\
\hline PCB19 & $4.92 \mathrm{E}+04$ & $3.81 \mathrm{E}+03$ & $1.08 \mathrm{E}+05$ & $1.04 \mathrm{E}+05$ \\
\hline PCB22 & $1.45 \mathrm{E}+05$ & $4.01 \mathrm{E}+03$ & $1.68 \mathrm{E}+05$ & $8.23 \mathrm{E}+03$ \\
\hline PCB28/PCB31 & $2.16 \mathrm{E}+05$ & $6.33 \mathrm{E}+03$ & $2.28 \mathrm{E}+05$ & $1.45 \mathrm{E}+04$ \\
\hline PCB33 & $9.96 \mathrm{E}+04$ & $2.54 \mathrm{E}+03$ & $1.13 \mathrm{E}+05$ & $5.03 \mathrm{E}+03$ \\
\hline PCB37 & $4.06 \mathrm{E}+04$ & $1.63 \mathrm{E}+03$ & $1.57 \mathrm{E}+05$ & $9.01 \mathrm{E}+03$ \\
\hline PCB40 & $1.35 \mathrm{E}+05$ & $2.75 \mathrm{E}+03$ & $1.57 \mathrm{E}+05$ & $9.01 \mathrm{E}+03$ \\
\hline PCB41 & $1.33 \mathrm{E}+05$ & $3.15 \mathrm{E}+03$ & $1.49 \mathrm{E}+05$ & $7.99 \mathrm{E}+03$ \\
\hline PCB44 & $7.51 \mathrm{E}+04$ & $4.59 \mathrm{E}+03$ & $1.43 \mathrm{E}+05$ & $9.15 \mathrm{E}+03$ \\
\hline PCB49 & $9.37 \mathrm{E}+04$ & $9.71 \mathrm{E}+02$ & $1.10 \mathrm{E}+05$ & $6.90 \mathrm{E}+03$ \\
\hline PCB52 & $8.05 \mathrm{E}+04$ & $1.76 \mathrm{E}+03$ & $9.12 \mathrm{E}+04$ & $4.48 \mathrm{E}+03$ \\
\hline PCB54 & $4.80 \mathrm{E}+04$ & $3.14 \mathrm{E}+03$ & $5.69 \mathrm{E}+04$ & $3.93 \mathrm{E}+03$ \\
\hline PCB66 & $9.50 \mathrm{E}+04$ & $1.43 \mathrm{E}+04$ & $1.51 \mathrm{E}+05$ & $8.50 \mathrm{E}+03$ \\
\hline PCB70 & $1.12 \mathrm{E}+05$ & $2.88 \mathrm{E}+04$ & $1.37 \mathrm{E}+05$ & $9.22 \mathrm{E}+03$ \\
\hline PCB74* & $\mathrm{n} / \mathrm{a}$ & $\mathrm{n} / \mathrm{a}$ & $1.63 \mathrm{E}+05$ & $1.54 \mathrm{E}+04$ \\
\hline PCB77 & $6.82 \mathrm{E}+04$ & $2.75 \mathrm{E}+03$ & $7.89 \mathrm{E}+04$ & $2.37 \mathrm{E}+03$ \\
\hline PCB81 & $8.46 \mathrm{E}+04$ & $1.06 \mathrm{E}+04$ & $1.09 \mathrm{E}+05$ & $4.12 \mathrm{E}+03$ \\
\hline PCB84 & $1.20 \mathrm{E}+05$ & $1.33 \mathrm{E}+04$ & $1.41 \mathrm{E}+05$ & $8.27 \mathrm{E}+03$ \\
\hline PCB85 & $1.87 \mathrm{E}+05$ & $2.86 \mathrm{E}+04$ & $2.31 \mathrm{E}+05$ & $7.81 \mathrm{E}+04$ \\
\hline PCB87 & $1.82 \mathrm{E}+05$ & $6.88 \mathrm{E}+03$ & $2.21 \mathrm{E}+05$ & $1.41 \mathrm{E}+04$ \\
\hline PCB90/PCB101 & $2.53 \mathrm{E}+05$ & $2.82 \mathrm{E}+04$ & $3.14 \mathrm{E}+05$ & $1.66 \mathrm{E}+04$ \\
\hline PCB95 & $1.19 \mathrm{E}+05$ & $4.91 \mathrm{E}+04$ & $1.16 \mathrm{E}+05$ & $9.93 \mathrm{E}+03$ \\
\hline PCB97 & $1.28 \mathrm{E}+05$ & $1.40 \mathrm{E}+04$ & $1.54 \mathrm{E}+05$ & $7.83 \mathrm{E}+03$ \\
\hline PCB99** & $5.41 \mathrm{E}+04$ & $4.03 \mathrm{E}+04$ & $5.28 \mathrm{E}+05$ & $5.78 \mathrm{E}+04$ \\
\hline PCB104 & $7.36 \mathrm{E}+04$ & $1.93 \mathrm{E}+03$ & $8.90 \mathrm{E}+04$ & $4.71 \mathrm{E}+03$ \\
\hline PCB105 & $2.14 \mathrm{E}+05$ & $7.30 \mathrm{E}+03$ & $2.44 \mathrm{E}+05$ & $1.13 \mathrm{E}+04$ \\
\hline PCB110 & $1.75 \mathrm{E}+05$ & $3.51 \mathrm{E}+03$ & $2.48 \mathrm{E}+05$ & $1.17 \mathrm{E}+04$ \\
\hline PCB114 & $\mathrm{n} / \mathrm{a}$ & $\mathrm{n} / \mathrm{a}$ & $2.88 \mathrm{E}+05$ & $4.62 \mathrm{E}+04$ \\
\hline PCB118 & $1.37 \mathrm{E}+05$ & $4.23 \mathrm{E}+03$ & $1.66 \mathrm{E}+05$ & $7.21 \mathrm{E}+03$ \\
\hline PCB119 & $1.78 \mathrm{E}+05$ & $7.05 \mathrm{E}+03$ & $2.02 \mathrm{E}+05$ & $1.33 \mathrm{E}+04$ \\
\hline PCB123 & $1.64 \mathrm{E}+05$ & $5.50 \mathrm{E}+03$ & $1.79 \mathrm{E}+05$ & $1.37 \mathrm{E}+04$ \\
\hline PCB126 & $1.10 \mathrm{E}+05$ & $5.24 \mathrm{E}+03$ & $1.30 \mathrm{E}+05$ & $3.75 \mathrm{E}+03$ \\
\hline
\end{tabular}


Table B.2 Repeatability- Peak Heights

\begin{tabular}{|c|c|c|c|c|}
\hline \multirow{2}{*}{$\begin{array}{c}\mathrm{N}=10 \text { Replicates } \\
\text { Name }\end{array}$} & \multicolumn{2}{|c|}{$\mathbf{L N}_{2}$ Modulator } & \multicolumn{2}{|c|}{$\begin{array}{c}\begin{array}{c}\text { University of Waterloo } \\
\text { Modulator }\end{array} \\
\end{array}$} \\
\hline & Avg. & $\begin{array}{c}\text { Std. } \\
\text { Deviation }\end{array}$ & Avg. & $\begin{array}{c}\text { Std. } \\
\text { Deviation }\end{array}$ \\
\hline PCB128 & $2.25 \mathrm{E}+05$ & $6.90 \mathrm{E}+03$ & $2.73 \mathrm{E}+05$ & $1.22 \mathrm{E}+04$ \\
\hline PCB129 & $2.05 \mathrm{E}+05$ & $5.48 \mathrm{E}+03$ & $2.41 \mathrm{E}+05$ & $1.11 \mathrm{E}+04$ \\
\hline PCB135 & $1.27 \mathrm{E}+05$ & $2.70 \mathrm{E}+03$ & $1.53 \mathrm{E}+05$ & $7.27 \mathrm{E}+03$ \\
\hline PCB137 & $2.25 \mathrm{E}+05$ & $6.50 \mathrm{E}+03$ & $2.63 \mathrm{E}+05$ & $1.40 \mathrm{E}+04$ \\
\hline PCB138 & $1.90 \mathrm{E}+05$ & $6.29 \mathrm{E}+03$ & $2.19 \mathrm{E}+05$ & $1.12 \mathrm{E}+04$ \\
\hline PCB141 & $2.54 \mathrm{E}+05$ & $7.29 \mathrm{E}+03$ & $2.94 \mathrm{E}+05$ & $1.47 \mathrm{E}+04$ \\
\hline PCB149 & $1.12 \mathrm{E}+05$ & $2.56 \mathrm{E}+03$ & $1.53 \mathrm{E}+05$ & $9.52 \mathrm{E}+03$ \\
\hline PCB151 & $1.29 \mathrm{E}+05$ & $3.24 \mathrm{E}+03$ & $1.75 \mathrm{E}+05$ & $1.69 \mathrm{E}+04$ \\
\hline PCB153 & $1.61 \mathrm{E}+05$ & $1.57 \mathrm{E}+04$ & $1.80 \mathrm{E}+05$ & $2.86 \mathrm{E}+04$ \\
\hline PCB155 & $1.09 \mathrm{E}+05$ & $2.40 \mathrm{E}+03$ & $1.48 \mathrm{E}+05$ & $5.64 \mathrm{E}+04$ \\
\hline PCB156 & $2.49 \mathrm{E}+05$ & $1.40 \mathrm{E}+04$ & $2.94 \mathrm{E}+05$ & $1.40 \mathrm{E}+04$ \\
\hline PCB157 & $2.17 \mathrm{E}+05$ & $8.44 \mathrm{E}+03$ & $2.76 \mathrm{E}+05$ & $1.39 \mathrm{E}+04$ \\
\hline PCB158 & $2.43 \mathrm{E}+05$ & $7.62 \mathrm{E}+03$ & $2.77 \mathrm{E}+05$ & $6.54 \mathrm{E}+04$ \\
\hline PCB167*** & $1.89 \mathrm{E}+05$ & $6.67 \mathrm{E}+03$ & $\mathrm{n} / \mathrm{a}$ & $\mathrm{n} / \mathrm{a}$ \\
\hline PCB168 & $1.70 \mathrm{E}+05$ & $1.52 \mathrm{E}+04$ & $2.11 \mathrm{E}+05$ & $2.99 \mathrm{E}+04$ \\
\hline PCB169 & $1.63 \mathrm{E}+05$ & $9.44 \mathrm{E}+03$ & $2.24 \mathrm{E}+05$ & $1.29 \mathrm{E}+04$ \\
\hline PCB170 & $2.53 \mathrm{E}+05$ & $9.88 \mathrm{E}+03$ & $2.95 \mathrm{E}+05$ & $1.20 \mathrm{E}+04$ \\
\hline PCB174 & $1.93 \mathrm{E}+05$ & $5.71 \mathrm{E}+03$ & $2.26 \mathrm{E}+05$ & $1.08 \mathrm{E}+04$ \\
\hline PCB177 & $1.83 \mathrm{E}+05$ & $5.95 \mathrm{E}+03$ & $2.20 \mathrm{E}+05$ & $1.11 \mathrm{E}+04$ \\
\hline PCB178 & $1.69 \mathrm{E}+05$ & $4.05 \mathrm{E}+03$ & $1.96 \mathrm{E}+05$ & $1.05 \mathrm{E}+04$ \\
\hline PCB180 & $2.61 \mathrm{E}+05$ & $9.99 \mathrm{E}+03$ & $2.60 \mathrm{E}+05$ & $1.94 \mathrm{E}+04$ \\
\hline PCB183 & $2.04 \mathrm{E}+05$ & $6.06 \mathrm{E}+03$ & $2.29 \mathrm{E}+05$ & $1.74 \mathrm{E}+04$ \\
\hline PCB187 & $1.75 \mathrm{E}+05$ & $5.66 \mathrm{E}+03$ & $1.87 \mathrm{E}+05$ & $2.09 \mathrm{E}+04$ \\
\hline PCB188 & $1.33 \mathrm{E}+05$ & $3.17 \mathrm{E}+03$ & $1.73 \mathrm{E}+05$ & $4.02 \mathrm{E}+04$ \\
\hline PCB189 & $3.00 \mathrm{E}+05$ & $1.49 \mathrm{E}+04$ & $2.40 \mathrm{E}+05$ & $7.28 \mathrm{E}+04$ \\
\hline PCB191 & $2.87 \mathrm{E}+05$ & $1.12 \mathrm{E}+04$ & $2.85 \mathrm{E}+05$ & $7.85 \mathrm{E}+04$ \\
\hline PCB193 & $2.49 \mathrm{E}+05$ & $9.29 \mathrm{E}+03$ & $2.56 \mathrm{E}+05$ & $2.83 \mathrm{E}+04$ \\
\hline PCB194 & $3.22 \mathrm{E}+05$ & $1.45 \mathrm{E}+04$ & $3.07 \mathrm{E}+05$ & $1.29 \mathrm{E}+04$ \\
\hline PCB199 & $2.35 \mathrm{E}+05$ & $8.92 \mathrm{E}+03$ & $2.38 \mathrm{E}+05$ & $1.02 \mathrm{E}+04$ \\
\hline PCB200 & $2.42 \mathrm{E}+05$ & $7.05 \mathrm{E}+03$ & $2.78 \mathrm{E}+05$ & $1.46 \mathrm{E}+04$ \\
\hline PCB201 & $1.71 \mathrm{E}+05$ & $4.65 \mathrm{E}+03$ & $1.90 \mathrm{E}+05$ & $1.09 \mathrm{E}+04$ \\
\hline PCB202 & $1.49 \mathrm{E}+05$ & $4.19 \mathrm{E}+03$ & $1.65 \mathrm{E}+05$ & $8.87 \mathrm{E}+03$ \\
\hline PCB203 & $3.42 \mathrm{E}+05$ & $1.16 \mathrm{E}+04$ & $3.07 \mathrm{E}+05$ & $1.34 \mathrm{E}+04$ \\
\hline PCB205 & $3.26 \mathrm{E}+05$ & $1.58 \mathrm{E}+04$ & $2.84 \mathrm{E}+05$ & $1.19 \mathrm{E}+04$ \\
\hline РCB206 & $3.25 \mathrm{E}+05$ & $1.60 \mathrm{E}+04$ & $3.11 \mathrm{E}+05$ & $1.51 \mathrm{E}+04$ \\
\hline РCB207 & $3.05 \mathrm{E}+05$ & $9.38 \mathrm{E}+03$ & $2.76 \mathrm{E}+05$ & $1.21 \mathrm{E}+04$ \\
\hline PCB208 & $2.75 \mathrm{E}+05$ & $9.37 \mathrm{E}+03$ & $2.46 \mathrm{E}+05$ & $1.06 \mathrm{E}+04$ \\
\hline
\end{tabular}


Table B.2 Repeatability- Peak Heights

\begin{tabular}{|c|c|c|c|c|}
\hline \multirow{2}{*}{$\frac{\mathrm{N}=10 \text { Replicates }}{\text { Name }}$} & \multicolumn{2}{|c|}{$\mathbf{L N}_{2}$ Modulator } & \multicolumn{2}{|c|}{$\begin{array}{c}\begin{array}{c}\text { University of Waterloo } \\
\text { Modulator }\end{array} \\
\end{array}$} \\
\hline & Avg. & $\begin{array}{c}\text { Std. } \\
\text { Deviation }\end{array}$ & Avg. & $\begin{array}{c}\text { Std. } \\
\text { Deviation }\end{array}$ \\
\hline PCB209 & $2.70 \mathrm{E}+05$ & $1.21 \mathrm{E}+04$ & $2.32 \mathrm{E}+05$ & $1.18 \mathrm{E}+04$ \\
\hline HCB & $3.98 \mathrm{E}+05$ & $8.26 \mathrm{E}+03$ & $3.79 \mathrm{E}+05$ & $2.38 \mathrm{E}+04$ \\
\hline HCBD & $7.69 \mathrm{E}+05$ & $1.10 \mathrm{E}+04$ & $\mathrm{n} / \mathrm{a}$ & $\mathrm{n} / \mathrm{a}$ \\
\hline HCE & $8.43 \mathrm{E}+05$ & $2.44 \mathrm{E}+04$ & $\mathrm{n} / \mathrm{a}$ & $\mathrm{n} / \mathrm{a}$ \\
\hline 1,2,3,4-TCB & $2.27 \mathrm{E}+05$ & $5.55 \mathrm{E}+03$ & $2.40 \mathrm{E}+05$ & $1.59 \mathrm{E}+04$ \\
\hline $1,2,3,5-/ 1,2,4,5-\mathrm{TCB}$ & $2.70 \mathrm{E}+05$ & $1.69 \mathrm{E}+04$ & $2.84 \mathrm{E}+05$ & $3.50 \mathrm{E}+04$ \\
\hline 1,2,3-ТCB & $1.28 \mathrm{E}+05$ & $3.91 \mathrm{E}+03$ & $1.25 \mathrm{E}+05$ & $1.81 \mathrm{E}+04$ \\
\hline 1,2,4-TCB & $7.12 \mathrm{E}+04$ & $1.42 \mathrm{E}+03$ & $7.02 \mathrm{E}+04$ & $9.12 \mathrm{E}+03$ \\
\hline 1,3,5-ТBB & $5.23 \mathrm{E}+05$ & $1.44 \mathrm{E}+04$ & $5.23 \mathrm{E}+05$ & $5.04 \mathrm{E}+04$ \\
\hline 1,3,5-ТCB & $8.62 \mathrm{E}+04$ & $1.98 \mathrm{E}+03$ & $7.01 \mathrm{E}+04$ & $1.73 \mathrm{E}+04$ \\
\hline 2,3,6-TCT & $1.15 \mathrm{E}+05$ & $2.58 \mathrm{E}+03$ & $1.13 \mathrm{E}+05$ & $1.17 \mathrm{E}+04$ \\
\hline 2,4,5-TCT & $6.62 \mathrm{E}+04$ & $3.38 \mathrm{E}+03$ & $6.94 \mathrm{E}+04$ & $5.96 \mathrm{E}+03$ \\
\hline P5CB & $3.16 \mathrm{E}+05$ & $4.78 \mathrm{E}+04$ & $3.73 \mathrm{E}+05$ & $2.76 \mathrm{E}+04$ \\
\hline A2,6-TCT & $3.68 \mathrm{E}+05$ & $1.23 \mathrm{E}+04$ & $3.98 \mathrm{E}+05$ & $4.25 \mathrm{E}+04$ \\
\hline A-BHC & $5.06 \mathrm{E}+05$ & $9.33 \mathrm{E}+03$ & $5.71 \mathrm{E}+05$ & $3.91 \mathrm{E}+04$ \\
\hline G-BHC & $4.08 \mathrm{E}+05$ & $9.80 \mathrm{E}+03$ & $4.69 \mathrm{E}+05$ & $3.23 \mathrm{E}+04$ \\
\hline A-CHLA** & $3.44 \mathrm{E}+05$ & $4.47 \mathrm{E}+03$ & $5.28 \mathrm{E}+05$ & $5.78 \mathrm{E}+04$ \\
\hline Oxy-CHLA & $2.79 \mathrm{E}+05$ & $7.93 \mathrm{E}+03$ & $3.59 \mathrm{E}+05$ & $2.40 \mathrm{E}+04$ \\
\hline Aldrin & $4.47 \mathrm{E}+05$ & $6.65 \mathrm{E}+03$ & $5.18 \mathrm{E}+05$ & $3.49 \mathrm{E}+04$ \\
\hline Endrin & $1.47 \mathrm{E}+05$ & $1.68 \mathrm{E}+04$ & $1.46 \mathrm{E}+05$ & $3.85 \mathrm{E}+04$ \\
\hline Dieldrin & $3.08 \mathrm{E}+05$ & $7.69 \mathrm{E}+03$ & $3.50 \mathrm{E}+05$ & $5.34 \mathrm{E}+04$ \\
\hline Endos 1 & $2.65 \mathrm{E}+05$ & $1.29 \mathrm{E}+04$ & $3.84 \mathrm{E}+05$ & $3.50 \mathrm{E}+04$ \\
\hline Endos 2 & $2.10 \mathrm{E}+05$ & $9.62 \mathrm{E}+03$ & $2.38 \mathrm{E}+05$ & $1.20 \mathrm{E}+04$ \\
\hline Endos S & $2.14 \mathrm{E}+05$ & $4.39 \mathrm{E}+03$ & $2.38 \mathrm{E}+05$ & $1.20 \mathrm{E}+04$ \\
\hline Heptachlor & $2.96 \mathrm{E}+05$ & $1.07 \mathrm{E}+04$ & $2.75 \mathrm{E}+05$ & $2.38 \mathrm{E}+04$ \\
\hline OCSTYR & $4.32 \mathrm{E}+05$ & $8.84 \mathrm{E}+03$ & $5.09 \mathrm{E}+05$ & $3.10 \mathrm{E}+04$ \\
\hline Trans-Nonachlor & $2.98 \mathrm{E}+05$ & $4.71 \mathrm{E}+04$ & $3.99 \mathrm{E}+05$ & $2.59 \mathrm{E}+04$ \\
\hline o,p'-DDT & $1.53 \mathrm{E}+05$ & $5.75 \mathrm{E}+03$ & $1.60 \mathrm{E}+05$ & $7.66 \mathrm{E}+03$ \\
\hline p,p'-DDD & $2.45 \mathrm{E}+05$ & $4.82 \mathrm{E}+03$ & $2.75 \mathrm{E}+05$ & $1.14 \mathrm{E}+04$ \\
\hline p,p'-DDE & $3.43 \mathrm{E}+05$ & $7.07 \mathrm{E}+03$ & $4.00 \mathrm{E}+05$ & $2.79 \mathrm{E}+04$ \\
\hline p,p'-DDT & $1.90 \mathrm{E}+05$ & $3.32 \mathrm{E}+04$ & $1.46 \mathrm{E}+05$ & $1.29 \mathrm{E}+04$ \\
\hline Mirex & $1.68 \mathrm{E}+05$ & $3.27 \mathrm{E}+03$ & $1.55 \mathrm{E}+05$ & $2.82 \mathrm{E}+03$ \\
\hline p-Mirex $* * *$ & $1.78 \mathrm{E}+05$ & $3.72 \mathrm{E}+03$ & $\mathrm{n} / \mathrm{a}$ & $\mathrm{n} / \mathrm{a}$ \\
\hline G-CHLA/PCB60* & $5.38 \mathrm{E}+05$ & $9.20 \mathrm{E}+03$ & $6.35 \mathrm{E}+05$ & $3.38 \mathrm{E}+04$ \\
\hline DMDT/PCB171 & $2.32 \mathrm{E}+05$ & $7.62 \mathrm{E}+03$ & $2.88 \mathrm{E}+05$ & $8.64 \mathrm{E}+03$ \\
\hline Cis-Nonachlor/PCB114 & $6.04 \mathrm{E}+05$ & $1.32 \mathrm{E}+04$ & $\mathrm{n} / \mathrm{a}$ & $\mathrm{n} / \mathrm{a}$ \\
\hline H-Epoxide/PCB74 & $4.51 \mathrm{E}+05$ & $7.61 \mathrm{E}+03$ & $\mathrm{n} / \mathrm{a}$ & $\mathrm{n} / \mathrm{a}$ \\
\hline
\end{tabular}


Table B.2 Repeatability- Peak Heights

\begin{tabular}{|c|c|c|c|c|}
\hline N=10 Replicates & \multicolumn{2}{|c|}{ LN2 Modulator } & \multicolumn{2}{|c|}{$\begin{array}{c}\text { University of Waterloo } \\
\text { Modulator }\end{array}$} \\
\hline Name & Avg. & $\begin{array}{c}\text { Std. } \\
\text { Deviation }\end{array}$ & Avg. & $\begin{array}{c}\text { Std. } \\
\text { Deviation }\end{array}$ \\
\hline H-Epoxide & n/a & n/a & $4.38 \mathrm{E}+05$ & $2.55 \mathrm{E}+04$ \\
\hline Cis-Nonachlor & $\mathrm{n} / \mathrm{a}$ & $\mathrm{n} / \mathrm{a}$ & $3.72 \mathrm{E}+05$ & $4.87 \mathrm{E}+04$ \\
\hline PCB99/Alpha-Chlordane & n/a & n/a & $5.28 \mathrm{E}+05$ & $5.78 \mathrm{E}+04$ \\
\hline PCB167/Photo-Mirex & n/a & n/a & $4.80 \mathrm{E}+05$ & $9.08 \mathrm{E}+04$ \\
\hline
\end{tabular}

*PCB74/H-epoxide coelutopn - $\mathrm{LN}_{2}$ modulator

**PCB99/alpha-chlordane coelution - UW modulator

***PCB167/photo-Mirex coelution - UW modulator 
Table B.3 Instrument Detection Limits for the GC $\times$ GC- $\mu$ ECD systems with the two modulators

\begin{tabular}{|c|c|c|c|c|c|c|c|c|}
\hline \multirow[b]{2}{*}{ Name } & \multicolumn{4}{|c|}{$\mathrm{LN}_{2}$ Modulator $(\mathrm{N}=9)$} & \multicolumn{4}{|c|}{ University of Waterloo Modulator $(\mathrm{N}=5)$} \\
\hline & $\underset{\text { (ng/mL) }}{\text { Avg. }}$ & $\begin{array}{c}\text { Std. } \\
\text { Deviation }\end{array}$ & $\begin{array}{c}\% \\
\text { RSD }\end{array}$ & $\begin{array}{c}\text { IDL } \\
(\mathbf{n g} / \mathbf{m L})\end{array}$ & $\underset{\text { (ng/mL) }}{\text { Avg. }}$ & $\begin{array}{c}\text { Std. } \\
\text { Deviation }\end{array}$ & $\begin{array}{c}\% \\
\text { RSD }\end{array}$ & $\begin{array}{c}\text { IDL } \\
(\mathrm{ng} / \mathrm{mL})\end{array}$ \\
\hline PCB4/PCB10 & 1.99 & 0.10 & 4.85 & 0.29 & 1.90 & 0.06 & 3.14 & 0.18 \\
\hline PCB6 & 0.97 & 0.10 & 10.17 & 0.30 & 0.99 & 0.18 & 18.32 & 0.55 \\
\hline PCB8 & 0.95 & 0.05 & 5.77 & 0.16 & 0.81 & 0.09 & 11.33 & 0.28 \\
\hline PCB15 & 1.07 & 0.08 & 7.79 & 0.25 & 0.78 & 0.11 & 14.45 & 0.34 \\
\hline PCB16 & 1.06 & 0.03 & 3.13 & 0.10 & 1.02 & 0.04 & 3.62 & 0.11 \\
\hline PCB18 & 1.05 & 0.02 & 1.99 & 0.06 & 0.89 & 0.05 & 5.15 & 0.14 \\
\hline PCB19 & 1.06 & 0.02 & 1.89 & 0.06 & 0.86 & 0.12 & 14.24 & 0.37 \\
\hline PCB22 & 0.90 & 0.03 & 3.14 & 0.08 & 1.07 & 0.04 & 3.74 & 0.12 \\
\hline PCB28/PCB31 & 1.78 & 0.03 & 1.08 & 0.08 & 2.07 & 0.07 & 3.26 & 0.20 \\
\hline PCB33 & 0.89 & 0.05 & 5.42 & 0.14 & 1.03 & 0.07 & 6.43 & 0.20 \\
\hline PCB37 & 1.01 & 0.11 & 11.14 & 0.34 & 1.04 & 0.06 & 6.12 & 0.19 \\
\hline PCB40 & 1.01 & 0.04 & 3.95 & 0.12 & 1.04 & 0.06 & 6.12 & 0.19 \\
\hline PCB41 & 1.08 & 0.03 & 3.23 & 0.10 & 1.03 & 0.03 & 2.87 & 0.09 \\
\hline PCB44 & 0.94 & 0.06 & 6.02 & 0.17 & 1.10 & 0.06 & 5.89 & 0.19 \\
\hline PCB49 & 0.88 & 0.05 & 5.27 & 0.14 & 0.96 & 0.07 & 7.27 & 0.21 \\
\hline PCB52 & 0.94 & 0.12 & 12.50 & 0.35 & 1.04 & 0.07 & 6.56 & 0.20 \\
\hline PCB54 & 1.06 & 0.09 & 8.34 & 0.26 & 0.90 & 0.04 & 3.95 & 0.11 \\
\hline PCB66 & 0.98 & 0.04 & 3.82 & 0.11 & 1.01 & 0.04 & 4.39 & 0.13 \\
\hline PCB70 & 0.98 & 0.07 & 7.17 & 0.21 & 0.96 & 0.05 & 5.37 & 0.16 \\
\hline PCB74* & $\mathrm{n} / \mathrm{a}$ & $\mathrm{n} / \mathrm{a}$ & $\mathrm{n} / \mathrm{a}$ & $\mathrm{n} / \mathrm{a}$ & 1.17 & 0.01 & 1.15 & 0.04 \\
\hline PCB77 & 1.01 & 0.04 & 3.91 & 0.12 & 1.01 & 0.11 & 11.03 & 0.34 \\
\hline PCB81 & 1.14 & 0.05 & 4.09 & 0.14 & 1.05 & 0.09 & 8.69 & 0.27 \\
\hline PCB84 & 0.97 & 0.04 & 3.63 & 0.11 & 1.01 & 0.04 & 4.05 & 0.12 \\
\hline PCB85 & 1.04 & 0.02 & 1.52 & 0.05 & 0.97 & 0.07 & 6.87 & 0.20 \\
\hline PCB87 & 1.07 & 0.07 & 6.13 & 0.20 & 1.25 & 0.03 & 2.36 & 0.09 \\
\hline PCB90/PCB101 & 2.04 & 0.11 & 5.40 & 0.33 & 2.12 & 0.03 & 1.31 & 0.08 \\
\hline PCB95 & 1.09 & 0.05 & 4.81 & 0.16 & 1.05 & 0.04 & 3.95 & 0.12 \\
\hline PCB97 & 1.07 & 0.07 & 6.13 & 0.20 & 1.03 & 0.08 & 7.39 & 0.23 \\
\hline PCB99** & $\mathrm{n} / \mathrm{a}$ & $\mathrm{n} / \mathrm{a}$ & $\mathrm{n} / \mathrm{a}$ & $\mathrm{n} / \mathrm{a}$ & 2.18 & 0.07 & 3.40 & 0.22 \\
\hline PCB104 & 0.94 & 0.06 & 5.95 & 0.17 & 0.86 & 0.06 & 7.03 & 0.18 \\
\hline PCB105 & 0.98 & 0.03 & 3.47 & 0.10 & 1.10 & 0.04 & 3.39 & 0.11 \\
\hline PCB110 & 0.97 & 0.03 & 3.03 & 0.09 & 0.99 & 0.04 & 3.57 & 0.11 \\
\hline PCB114 & $\mathrm{n} / \mathrm{a}$ & $\mathrm{n} / \mathrm{a}$ & $\mathrm{n} / \mathrm{a}$ & $\mathrm{n} / \mathrm{a}$ & 0.92 & 0.12 & 12.78 & 0.35 \\
\hline PCB118 & 1.07 & 0.03 & 3.02 & 0.10 & 1.12 & 0.23 & 20.10 & 0.68 \\
\hline PCB119 & 1.06 & 0.03 & 2.75 & 0.09 & 1.10 & 0.10 & 8.72 & 0.29 \\
\hline PCB123 & 1.00 & 0.04 & 3.99 & 0.12 & 1.09 & 0.29 & 26.26 & 0.86 \\
\hline PCB126 & 1.11 & 0.02 & 2.25 & 0.07 & 0.96 & 0.04 & 3.81 & 0.11 \\
\hline PCB128 & 0.91 & 0.05 & 5.63 & 0.15 & 1.05 & 0.02 & 2.23 & 0.07 \\
\hline
\end{tabular}


Table B.3 Instrument Detection Limits for the GC $\times$ GC- $\mu$ ECD systems with the two modulators

\begin{tabular}{|c|c|c|c|c|c|c|c|c|}
\hline \multirow[b]{2}{*}{ Name } & \multicolumn{4}{|c|}{$\mathrm{LN}_{2}$ Modulator $(\mathrm{N}=9)$} & \multicolumn{4}{|c|}{ University of Waterloo Modulator $(\mathrm{N}=5)$} \\
\hline & $\underset{(\text { Avg. }}{\text { AvL) }}$ & $\begin{array}{c}\text { Std. } \\
\text { Deviation }\end{array}$ & $\begin{array}{c}\% \\
\text { RSD }\end{array}$ & $\underset{(\mathbf{n g} / \mathbf{m L})}{\mathrm{IDL}}$ & $\underset{(\mathrm{ng} / \mathrm{mL})}{\text { Avg. }}$ & $\begin{array}{c}\text { Std. } \\
\text { Deviation }\end{array}$ & $\begin{array}{c}\% \\
\text { RSD }\end{array}$ & $\begin{array}{c}\text { IDL } \\
(\mathbf{n g} / \mathbf{m L})\end{array}$ \\
\hline PCB129 & 1.15 & 0.03 & 2.30 & 0.08 & 1.05 & 0.02 & 2.07 & 0.07 \\
\hline PCB135 & 1.11 & 0.05 & 4.20 & 0.14 & 0.95 & 0.14 & 14.30 & 0.41 \\
\hline PCB137 & 1.13 & 0.04 & 3.79 & 0.13 & 1.10 & 0.01 & 1.11 & 0.04 \\
\hline PCB138 & 1.12 & 0.03 & 2.36 & 0.08 & 0.93 & 0.04 & 4.30 & 0.12 \\
\hline PCB141 & 1.06 & 0.03 & 3.26 & 0.10 & 1.03 & 0.01 & 1.10 & 0.03 \\
\hline PCB149 & 0.98 & 0.03 & 3.28 & 0.10 & 1.05 & 0.03 & 3.11 & 0.10 \\
\hline PCB151 & 0.92 & 0.03 & 3.38 & 0.09 & 1.03 & 0.10 & 9.95 & 0.31 \\
\hline PCB153 & 1.00 & 0.04 & 4.08 & 0.12 & 0.89 & 0.04 & 4.63 & 0.12 \\
\hline PCB155 & 1.01 & 0.03 & 3.43 & 0.10 & 1.02 & 0.07 & 6.39 & 0.20 \\
\hline PCB156 & 1.05 & 0.05 & 4.47 & 0.14 & 1.10 & 0.01 & 1.11 & 0.04 \\
\hline PCB157 & 1.03 & 0.05 & 4.81 & 0.15 & 1.19 & 0.02 & 1.75 & 0.06 \\
\hline PCB158 & 1.09 & 0.03 & 2.54 & 0.08 & 1.09 & 0.03 & 2.93 & 0.10 \\
\hline PCB167**** & 1.00 & 0.03 & 2.54 & 0.08 & $\mathrm{n} / \mathrm{a}$ & $\mathrm{n} / \mathrm{a}$ & $\mathrm{n} / \mathrm{a}$ & $\mathrm{n} / \mathrm{a}$ \\
\hline PCB168 & 0.98 & 0.05 & 4.60 & 0.14 & 1.17 & 0.04 & 3.28 & 0.12 \\
\hline PCB169 & 1.08 & 0.04 & 3.89 & 0.13 & 1.06 & 0.03 & 3.22 & 0.10 \\
\hline PCB170 & 1.13 & 0.03 & 3.05 & 0.10 & 1.06 & 0.02 & 1.63 & 0.05 \\
\hline PCB174 & 1.10 & 0.03 & 2.44 & 0.08 & 1.05 & 0.05 & 4.76 & 0.15 \\
\hline PCB177 & 1.20 & 0.05 & 4.44 & 0.16 & 0.98 & 0.03 & 2.83 & 0.08 \\
\hline PCB178 & 1.07 & 0.11 & 10.40 & 0.33 & 0.93 & 0.06 & 6.18 & 0.17 \\
\hline PCB180 & 1.07 & 0.04 & 3.67 & 0.12 & 0.94 & 0.03 & 2.76 & 0.08 \\
\hline PCB183 & 0.96 & 0.03 & 3.06 & 0.09 & 1.01 & 0.02 & 2.38 & 0.07 \\
\hline PCB187 & 1.15 & 0.02 & 2.06 & 0.07 & 0.97 & 0.06 & 6.44 & 0.19 \\
\hline PCB188 & 0.98 & 0.06 & 5.78 & 0.17 & 0.95 & 0.07 & 7.73 & 0.22 \\
\hline PCB189 & 1.08 & 0.03 & 2.38 & 0.08 & 1.12 & 0.03 & 2.66 & 0.09 \\
\hline PCB191 & 1.07 & 0.06 & 5.37 & 0.17 & 1.08 & 0.02 & 1.73 & 0.06 \\
\hline PCB193 & no data & no data & no data & no data & 1.11 & 0.04 & 3.28 & 0.11 \\
\hline PCB194 & 1.08 & 0.03 & 3.02 & 0.10 & 1.05 & 0.03 & 2.56 & 0.08 \\
\hline PCB199 & 1.01 & 0.03 & 3.15 & 0.10 & 1.04 & 0.05 & 4.57 & 0.14 \\
\hline PCB200 & 1.07 & 0.03 & 2.82 & 0.09 & 1.06 & 0.06 & 5.94 & 0.19 \\
\hline PCB201 & 0.99 & 0.02 & 2.52 & 0.07 & 1.00 & 0.03 & 2.87 & 0.09 \\
\hline PCB202 & 1.04 & 0.04 & 3.38 & 0.11 & 0.91 & 0.05 & 5.10 & 0.14 \\
\hline PCB203 & 0.99 & 0.03 & 3.10 & 0.09 & 1.08 & 0.01 & 1.25 & 0.04 \\
\hline PCB205 & 1.12 & 0.03 & 2.56 & 0.09 & 1.10 & 0.09 & 8.05 & 0.26 \\
\hline РCB206 & 1.02 & 0.11 & 11.02 & 0.34 & 1.00 & 0.03 & 3.32 & 0.10 \\
\hline PCB207 & 1.05 & 0.03 & 2.95 & 0.09 & 1.06 & 0.03 & 2.50 & 0.08 \\
\hline PCB208 & 1.14 & 0.03 & 2.58 & 0.09 & 1.04 & 0.03 & 2.88 & 0.09 \\
\hline PCB209 & 1.05 & 0.02 & 2.06 & 0.07 & 1.13 & 0.02 & 1.45 & 0.05 \\
\hline HCB & 0.83 & 0.03 & 3.24 & 0.08 & 1.11 & 0.02 & 1.76 & 0.06 \\
\hline
\end{tabular}


Table B.3 Instrument Detection Limits for the GC $\times$ GC- $\mu$ ECD systems with the two modulators

\begin{tabular}{|c|c|c|c|c|c|c|c|c|}
\hline \multirow[b]{2}{*}{ Name } & \multicolumn{4}{|c|}{$\mathrm{LN}_{2}$ Modulator $(\mathrm{N}=9)$} & \multicolumn{4}{|c|}{ University of Waterloo Modulator $(\mathrm{N}=5)$} \\
\hline & $\underset{(\mathbf{n g} / \mathbf{m L})}{\text { Avg. }}$ & $\begin{array}{c}\text { Std. } \\
\text { Deviation }\end{array}$ & $\begin{array}{c}\% \\
\text { RSD }\end{array}$ & $\underset{(\mathbf{n g} / \mathbf{m L})}{\mathrm{IDL}}$ & $\underset{(\mathbf{n g} / \mathbf{m L})}{\operatorname{Avg})}$ & $\begin{array}{c}\text { Std. } \\
\text { Deviation }\end{array}$ & $\begin{array}{c}\% \\
\text { RSD }\end{array}$ & $\begin{array}{c}\text { IDL } \\
(\mathbf{n g} / \mathbf{m L})\end{array}$ \\
\hline HCBD & 1.10 & 0.04 & 4.04 & 0.13 & $\mathrm{n} / \mathrm{a}$ & $\mathrm{n} / \mathrm{a}$ & $\mathrm{n} / \mathrm{a}$ & $\mathrm{n} / \mathrm{a}$ \\
\hline HCE & 1.21 & 0.14 & 11.33 & 0.41 & $\mathrm{n} / \mathrm{a}$ & $\mathrm{n} / \mathrm{a}$ & $\mathrm{n} / \mathrm{a}$ & $\mathrm{n} / \mathrm{a}$ \\
\hline $1,2,3,4-$ TCB & 1.09 & 0.09 & 8.31 & 0.27 & 1.07 & 0.09 & 8.00 & 0.26 \\
\hline $\begin{array}{c}1,2,3,5-/ 1,2,4,5- \\
\text { TCB }\end{array}$ & 1.86 & 0.09 & 4.97 & 0.28 & 2.09 & 0.06 & 3.11 & 0.19 \\
\hline 1,2,3-TCB & 1.05 & 0.15 & 14.06 & 0.44 & 0.89 & 0.04 & 5.06 & 0.13 \\
\hline $1,2,4-\mathrm{TCB}$ & 0.92 & 0.10 & 11.28 & 0.31 & 0.68 & 0.15 & 21.78 & 0.45 \\
\hline 1,3,5-TBB & 1.00 & 0.02 & 1.96 & 0.06 & 1.08 & 0.03 & 3.14 & 0.10 \\
\hline 1,3,5-TCB & 1.05 & 0.12 & 10.99 & 0.35 & 1.31 & 0.19 & 14.62 & 0.57 \\
\hline 2,3,6-ТCT & 1.19 & 0.09 & 7.66 & 0.27 & 0.81 & 0.06 & 7.78 & 0.19 \\
\hline 2,4,5-TCT & 0.90 & 0.04 & 4.84 & 0.13 & 0.98 & 0.14 & 14.71 & 0.43 \\
\hline P5CB & 0.87 & 0.06 & 6.38 & 0.17 & 1.14 & 0.02 & 1.92 & 0.07 \\
\hline A2,6-TCT & 0.85 & 0.03 & 3.05 & 0.08 & 1.09 & 0.09 & 8.53 & 0.28 \\
\hline A-BHC & 0.89 & 0.04 & 4.11 & 0.11 & 1.30 & 0.02 & 1.54 & 0.06 \\
\hline G-BHC & 0.77 & 0.01 & 1.52 & 0.04 & 1.20 & 0.06 & 5.33 & 0.19 \\
\hline A-CHLA** & 1.00 & 0.03 & 3.14 & 0.09 & 2.18 & 0.07 & 3.40 & 0.22 \\
\hline Oxy-CHLA & 1.03 & 0.02 & 2.05 & 0.07 & 1.05 & 0.08 & 7.65 & 0.24 \\
\hline Aldrin & 1.05 & 0.03 & 3.14 & 0.10 & 1.21 & 0.04 & 3.22 & 0.12 \\
\hline Endrin & 1.10 & 0.02 & 1.42 & 0.05 & 1.30 & 0.05 & 3.67 & 0.14 \\
\hline Dieldrin & 0.97 & 0.03 & 2.80 & 0.09 & 1.05 & 0.08 & 7.70 & 0.24 \\
\hline Endos 1 & 0.94 & 0.02 & 2.22 & 0.06 & 1.19 & 0.02 & 2.09 & 0.07 \\
\hline Endos 2 & 0.99 & 0.03 & 2.71 & 0.08 & 1.16 & 0.11 & 9.65 & 0.34 \\
\hline Endos S & 0.74 & 0.03 & 3.17 & 0.09 & 1.16 & 0.11 & 9.65 & 0.34 \\
\hline Heptachlor & 0.85 & 0.02 & 2.71 & 0.06 & 1.08 & 0.34 & 31.55 & 1.02 \\
\hline OCSTYR & 0.86 & 0.02 & 2.03 & 0.05 & 1.15 & 0.04 & 3.10 & 0.11 \\
\hline Trans-Nonachlor & 0.98 & 0.02 & 1.88 & 0.05 & 1.11 & 0.06 & 4.98 & 0.17 \\
\hline o,p'-DDT & 1.31 & 0.04 & 4.02 & 0.12 & 0.99 & 0.17 & 17.05 & 0.51 \\
\hline p,p'-DDD & 0.87 & 0.14 & 10.76 & 0.42 & 1.17 & 0.02 & 2.04 & 0.07 \\
\hline p,p'-DDE & 1.02 & 0.04 & 4.17 & 0.11 & 1.23 & 0.03 & 2.42 & 0.09 \\
\hline p,p'-DDT & 1.40 & 0.02 & 2.43 & 0.07 & 1.17 & 0.10 & 8.74 & 0.31 \\
\hline Mirex & 0.82 & 0.05 & 3.50 & 0.15 & 1.08 & 0.09 & 8.39 & 0.27 \\
\hline p-Mirex $* * *$ & 0.97 & 0.02 & 1.97 & 0.05 & $\mathrm{n} / \mathrm{a}$ & $\mathrm{n} / \mathrm{a}$ & $\mathrm{n} / \mathrm{a}$ & $\mathrm{n} / \mathrm{a}$ \\
\hline G-CHLA/PCB60* & 2.21 & 0.05 & 2.40 & 0.16 & 2.12 & 0.02 & 0.71 & 0.05 \\
\hline DMDT/PCB171 & 2.01 & 0.03 & 1.73 & 0.10 & 2.35 & 0.12 & 5.08 & 0.36 \\
\hline $\begin{array}{c}\text { Cis- } \\
\text { Nonachlor/PCB114 } \\
\end{array}$ & 1.89 & 0.06 & 3.26 & 0.17 & $\mathrm{n} / \mathrm{a}$ & $\mathrm{n} / \mathrm{a}$ & $\mathrm{n} / \mathrm{a}$ & $\mathrm{n} / \mathrm{a}$ \\
\hline H-Epoxide/PCB74 & 1.96 & 0.04 & 1.81 & 0.10 & $\mathrm{n} / \mathrm{a}$ & $\mathrm{n} / \mathrm{a}$ & $\mathrm{n} / \mathrm{a}$ & $\mathrm{n} / \mathrm{a}$ \\
\hline H-Epoxide & $\mathrm{n} / \mathrm{a}$ & $\mathrm{n} / \mathrm{a}$ & $\mathrm{n} / \mathrm{a}$ & $\mathrm{n} / \mathrm{a}$ & 1.19 & 0.03 & 2.40 & 0.09 \\
\hline Cis-Nonachlor & $\mathrm{n} / \mathrm{a}$ & $\mathrm{n} / \mathrm{a}$ & $\mathrm{n} / \mathrm{a}$ & $\mathrm{n} / \mathrm{a}$ & 1.02 & 0.12 & 12.09 & 0.37 \\
\hline
\end{tabular}


Table B.3 Instrument Detection Limits for the GC $\times$ GC- $\mu$ ECD systems with the two modulators

\begin{tabular}{|c|c|c|c|c|c|c|c|c|}
\hline \multirow{2}{*}{ Name } & \multicolumn{3}{|c|}{ LN2 Modulator (N=9) } & \multicolumn{3}{c|}{ University of Waterloo Modulator (N=5) } \\
\cline { 2 - 9 } & $\begin{array}{c}\text { Avg. } \\
\text { (ng/mL) }\end{array}$ & $\begin{array}{c}\text { Std. } \\
\text { Deviation }\end{array}$ & $\begin{array}{c}\text { \% } \\
\text { RSD }\end{array}$ & $\begin{array}{c}\text { IDL } \\
(\mathbf{n g} / \mathbf{m L})\end{array}$ & $\begin{array}{c}\text { Avg. } \\
(\mathbf{n g} / \mathbf{m L})\end{array}$ & $\begin{array}{c}\text { Std. } \\
\text { Deviation }\end{array}$ & $\begin{array}{c}\text { \% } \\
\text { RSD }\end{array}$ & $\begin{array}{c}\text { IDL } \\
(\mathbf{n g} / \mathbf{m L})\end{array}$ \\
\hline $\begin{array}{c}\text { PCB99/Alpha- } \\
\text { Chlordane }\end{array}$ & $\mathrm{n} / \mathrm{a}$ & $\mathrm{n} / \mathrm{a}$ & $\mathrm{n} / \mathrm{a}$ & $\mathrm{n} / \mathrm{a}$ & 2.18 & 0.07 & 3.40 & 0.22 \\
\hline $\begin{array}{c}\text { PCB167/Photo- } \\
\text { Mirex }\end{array}$ & $\mathrm{n} / \mathrm{a}$ & $\mathrm{n} / \mathrm{a}$ & $\mathrm{n} / \mathrm{a}$ & $\mathrm{n} / \mathrm{a}$ & 2.13 & 0.05 & 2.44 & 0.16 \\
\hline
\end{tabular}

*PCB74/H-epoxide coelution - $\mathrm{LN}_{2}$ modulator

**PCB99/alpha-chlordane coelution - UW

modulator

***PCB167/photo-Mirex coelution - UW

modulator 
Table B.4 Repeatability- Calculated concentration of PCB/OC/CB standards

\begin{tabular}{|c|c|c|c|c|c|c|c|}
\hline \multirow{2}{*}{$\begin{array}{c}\mathrm{N}=10 \text { Replicates } \\
\text { Name }\end{array}$} & \multirow{2}{*}{$\begin{array}{c}\text { Design } \\
(\mathbf{n g} / \mathrm{mL})\end{array}$} & \multicolumn{3}{|c|}{$\mathbf{L N}_{2}$ Modulator } & \multicolumn{3}{|c|}{ University of Waterloo Modulator } \\
\hline & & $\underset{(\mathbf{n g} / \mathbf{m L})}{\operatorname{Avg})}$ & \% RSD & $\begin{array}{c}\text { Std. } \\
\text { Deviation }\end{array}$ & $\underset{(\mathbf{n g} / \mathbf{m L})}{\operatorname{Avg} .}$ & \% RSD & $\begin{array}{c}\text { Std. } \\
\text { Deviation }\end{array}$ \\
\hline PCB4/PCB10 & 40 & 47.6 & 7.0 & 3.4 & 41.0 & 2.7 & 1.1 \\
\hline PCB6 & 20 & 23.0 & 3.9 & 0.9 & 20.2 & 4.0 & 0.8 \\
\hline PCB8 & 20 & 21.2 & 2.7 & 0.6 & 20.3 & 3.1 & 0.6 \\
\hline PCB15 & 20 & 21.7 & 7.7 & 1.7 & 21.1 & 5.2 & 1.1 \\
\hline PCB16 & 20 & 21.3 & 2.2 & 0.5 & 21.8 & 24.6 & 5.4 \\
\hline PCB18 & 20 & 20.4 & 2.9 & 0.6 & 21.1 & 2.3 & 0.5 \\
\hline PCB19 & 20 & 20.5 & 8.2 & 1.7 & 20.3 & 6.8 & 1.4 \\
\hline PCB22 & 20 & 19.8 & 1.2 & 0.2 & 19.1 & 2.7 & 0.5 \\
\hline PCB28/PCB31 & 40 & 41.8 & 3.8 & 1.6 & 38.8 & 2.6 & 1.0 \\
\hline PCB33 & 20 & 20.1 & 1.2 & 0.2 & 19.7 & 3.3 & 0.6 \\
\hline PCB37 & 20 & 22.2 & 5.2 & 1.1 & 19.4 & 2.5 & 0.5 \\
\hline PCB40 & 20 & 22.6 & 3.2 & 0.7 & 19.4 & 2.5 & 0.5 \\
\hline PCB41 & 20 & 20.9 & 2.0 & 0.4 & 19.3 & 3.1 & 0.6 \\
\hline PCB44 & 20 & 15.4 & 11.3 & 1.7 & 20.0 & 7.0 & 1.4 \\
\hline PCB49 & 20 & 20.3 & 3.0 & 0.6 & 19.2 & 3.9 & 0.7 \\
\hline PCB52 & 20 & 20.5 & 0.7 & 0.1 & 20.1 & 3.4 & 0.7 \\
\hline PCB54 & 20 & 21.2 & 7.4 & 1.6 & 20.4 & 4.1 & 0.8 \\
\hline PCB66 & 20 & 19.9 & 6.6 & 1.3 & 19.0 & 6.6 & 1.3 \\
\hline PCB70 & 20 & 22.0 & 10.7 & 2.4 & 19.8 & 7.5 & 1.5 \\
\hline PCB74* & 20 & $\mathrm{n} / \mathrm{a}$ & $\mathrm{n} / \mathrm{a}$ & $\mathrm{n} / \mathrm{a}$ & 20.1 & 9.2 & 1.9 \\
\hline PCB77 & 20 & 20.6 & 3.3 & 0.7 & 19.1 & 6.0 & 1.1 \\
\hline PCB81 & 20 & 17.9 & 2.2 & 0.4 & 18.9 & 4.2 & 0.8 \\
\hline PCB84 & 20 & 23.0 & 3.0 & 0.7 & 19.8 & 3.0 & 0.6 \\
\hline PCB85 & 20 & 23.2 & 3.8 & 0.9 & 19.6 & 21.8 & 4.3 \\
\hline PCB87 & 20 & 19.2 & 4.1 & 0.8 & 19.0 & 4.4 & 0.8 \\
\hline PCB90/PCB101 & 40 & 43.2 & 1.2 & 0.5 & 38.2 & 2.6 & 1.0 \\
\hline PCB95 & 20 & 18.8 & 8.7 & 1.6 & 19.8 & 4.4 & 0.9 \\
\hline PCB97 & 20 & 20.0 & 1.2 & 0.2 & 18.9 & 3.2 & 0.6 \\
\hline PCB99** & 20 & 17.8 & 3.7 & 0.7 & $\mathrm{n} / \mathrm{a}$ & $\mathrm{n} / \mathrm{a}$ & $\mathrm{n} / \mathrm{a}$ \\
\hline PCB104 & 20 & 19.1 & 2.5 & 0.5 & 20.0 & 3.4 & 0.7 \\
\hline PCB105 & 20 & 19.2 & 1.9 & 0.4 & 18.8 & 3.6 & 0.7 \\
\hline PCB110 & 20 & 19.1 & 2.5 & 0.5 & 20.2 & 4.3 & 0.9 \\
\hline PCB114 & 20 & $\mathrm{n} / \mathrm{a}$ & $\mathrm{n} / \mathrm{a}$ & $\mathrm{n} / \mathrm{a}$ & 21.3 & 13.2 & 2.8 \\
\hline PCB118 & 20 & 18.0 & 5.8 & 1.0 & 21.1 & 5.3 & 1.1 \\
\hline PCB119 & 20 & 20.7 & 3.3 & 0.7 & 21.4 & 5.7 & 1.2 \\
\hline PCB123 & 20 & 21.4 & 3.8 & 0.8 & 21.4 & 6.2 & 1.3 \\
\hline PCB126 & 20 & 20.2 & 4.2 & 0.8 & 19.5 & 5.1 & 1.0 \\
\hline PCB128 & 20 & 19.7 & 2.0 & 0.4 & 18.8 & 3.7 & 0.7 \\
\hline PCB129 & 20 & 23.1 & 2.9 & 0.7 & 18.4 & 3.7 & 0.7 \\
\hline PCB135 & 20 & 19.5 & 15.3 & 3.0 & 20.6 & 6.1 & 1.3 \\
\hline
\end{tabular}


Table B.4 Repeatability- Calculated concentration of PCB/OC/CB standards

\begin{tabular}{|c|c|c|c|c|c|c|c|}
\hline \multirow{2}{*}{$\begin{array}{c}\mathrm{N}=10 \text { Replicates } \\
\text { Name }\end{array}$} & \multirow{2}{*}{$\begin{array}{c}\text { Design } \\
(\mathbf{n g} / \mathbf{m L})\end{array}$} & \multicolumn{3}{|c|}{$\mathbf{L N}_{2}$ Modulator } & \multicolumn{3}{|c|}{ University of Waterloo Modulator } \\
\hline & & $\underset{(\mathbf{n g} / \mathbf{m L})}{\operatorname{Avg})}$ & \% RSD & $\begin{array}{c}\text { Std. } \\
\text { Deviation }\end{array}$ & $\underset{(\mathbf{n g} / \mathbf{m L})}{\operatorname{Avg} .}$ & \% RSD & $\begin{array}{c}\text { Std. } \\
\text { Deviation }\end{array}$ \\
\hline PCB137 & 20 & 22.5 & 6.3 & 1.4 & 19.0 & 4.7 & 0.9 \\
\hline PCB138 & 20 & 20.2 & 2.9 & 0.6 & 18.8 & 4.8 & 0.9 \\
\hline PCB141 & 20 & 20.7 & 3.6 & 0.7 & 19.1 & 3.2 & 0.6 \\
\hline PCB149 & 20 & 19.0 & 2.4 & 0.5 & 18.4 & 3.2 & 0.6 \\
\hline PCB151 & 20 & 20.3 & 13.8 & 2.8 & 21.2 & 6.6 & 1.4 \\
\hline PCB153 & 20 & 22.8 & 7.8 & 1.8 & 18.8 & 12.9 & 2.4 \\
\hline PCB155 & 20 & 20.3 & 0.6 & 0.1 & 19.7 & 4.1 & 0.8 \\
\hline PCB156 & 20 & 18.9 & 4.0 & 0.8 & 18.4 & 4.1 & 0.7 \\
\hline PCB157 & 20 & 18.9 & 2.4 & 0.5 & 19.5 & 4.7 & 0.9 \\
\hline PCB158 & 20 & 20.0 & 4.0 & 0.8 & 19.5 & 3.9 & 0.8 \\
\hline PCB167**** & 20 & 21.2 & 3.8 & 0.8 & $\mathrm{n} / \mathrm{a}$ & $\mathrm{n} / \mathrm{a}$ & $\mathrm{n} / \mathrm{a}$ \\
\hline PCB168 & 20 & 17.4 & 8.0 & 1.4 & 20.7 & 14.1 & 2.9 \\
\hline PCB169 & 20 & 20.6 & 7.7 & 1.6 & 18.3 & 3.6 & 0.7 \\
\hline PCB170 & 20 & 19.3 & 2.8 & 0.5 & 18.8 & 3.9 & 0.7 \\
\hline PCB174 & 20 & 20.5 & 1.7 & 0.4 & 19.1 & 3.0 & 0.6 \\
\hline PCB177 & 20 & 21.2 & 2.1 & 0.5 & 19.3 & 3.1 & 0.6 \\
\hline PCB178 & 20 & 21.8 & 2.6 & 0.6 & 20.6 & 2.6 & 0.5 \\
\hline PCB180 & 20 & 21.7 & 4.6 & 1.0 & 19.0 & 6.9 & 1.3 \\
\hline PCB183 & 20 & 20.6 & 2.0 & 0.4 & 19.5 & 6.6 & 1.3 \\
\hline PCB187 & 20 & 21.9 & 2.3 & 0.5 & 19.3 & 3.2 & 0.6 \\
\hline PCB188 & 20 & 20.5 & 2.5 & 0.5 & 19.2 & 4.0 & 0.8 \\
\hline PCB189 & 20 & 20.9 & 6.6 & 1.4 & 18.5 & 4.0 & 0.8 \\
\hline PCB191 & 20 & 21.5 & 3.6 & 0.8 & 19.0 & 8.5 & 1.6 \\
\hline РCB193 & 20 & 21.2 & 2.7 & 0.6 & 18.9 & 3.9 & 0.7 \\
\hline PCB194 & 20 & 21.7 & 6.5 & 1.4 & 18.7 & 3.7 & 0.7 \\
\hline PCB199 & 20 & 21.4 & 4.3 & 0.9 & 18.8 & 2.8 & 0.5 \\
\hline PCB200 & 20 & 20.8 & 2.1 & 0.4 & 20.1 & 2.3 & 0.5 \\
\hline PCB201 & 20 & 21.8 & 2.5 & 0.5 & 20.1 & 2.8 & 0.6 \\
\hline PCB202 & 20 & 21.5 & 1.9 & 0.4 & 19.6 & 3.3 & 0.7 \\
\hline PCB203 & 20 & 23.7 & 4.8 & 1.1 & 19.0 & 3.3 & 0.6 \\
\hline PCB205 & 20 & 20.3 & 6.2 & 1.2 & 18.9 & 3.2 & 0.6 \\
\hline PCB206 & 20 & 21.8 & 7.9 & 1.7 & 19.4 & 3.6 & 0.7 \\
\hline PCB207 & 20 & 23.4 & 5.3 & 1.2 & 18.8 & 3.1 & 0.6 \\
\hline PCB208 & 20 & 22.1 & 5.1 & 1.1 & 18.8 & 3.1 & 0.6 \\
\hline PCB209 & 20 & 20.7 & 6.9 & 1.4 & 18.7 & 4.2 & 0.8 \\
\hline HCB & 20 & 22.3 & 3.4 & 0.8 & 19.8 & 5.8 & 1.1 \\
\hline HCBD & 20 & 23.2 & 3.2 & 0.7 & $\mathrm{n} / \mathrm{a}$ & $\mathrm{n} / \mathrm{a}$ & $\mathrm{n} / \mathrm{a}$ \\
\hline HCE & 20 & 22.2 & 4.3 & 1.0 & $\mathrm{n} / \mathrm{a}$ & $\mathrm{n} / \mathrm{a}$ & $\mathrm{n} / \mathrm{a}$ \\
\hline 1,2,3,4-TCB & 20 & 21.3 & 3.7 & 0.8 & 21.0 & 4.7 & 1.0 \\
\hline $1,2,3,5-/ 1,2,4,5-\mathrm{TCB}$ & 40 & 39.9 & 6.3 & 2.5 & 40.5 & 6.4 & 2.6 \\
\hline
\end{tabular}


Table B.4 Repeatability- Calculated concentration of PCB/OC/CB standards

\begin{tabular}{|c|c|c|c|c|c|c|c|}
\hline \multirow{2}{*}{$\begin{array}{c}\mathrm{N}=10 \text { Replicates } \\
\text { Name }\end{array}$} & \multirow{2}{*}{$\begin{array}{c}\text { Design } \\
(\mathbf{n g} / \mathbf{m L})\end{array}$} & \multicolumn{3}{|c|}{$\mathbf{L N}_{2}$ Modulator } & \multicolumn{3}{|c|}{ University of Waterloo Modulator } \\
\hline & & $\underset{(\mathbf{n g} / \mathbf{m L})}{\operatorname{Avg} .}$ & \% RSD & $\begin{array}{c}\text { Std. } \\
\text { Deviation }\end{array}$ & $\underset{(\mathbf{n g} / \mathbf{m L})}{\operatorname{Avg.}}$ & \% RSD & $\begin{array}{c}\text { Std. } \\
\text { Deviation }\end{array}$ \\
\hline 1,2,3-TCB & 20 & 21.8 & 3.3 & 0.7 & 20.6 & 4.4 & 0.9 \\
\hline 1,2,4-ТCB & 20 & 22.6 & 2.0 & 0.4 & 19.7 & 6.7 & 1.3 \\
\hline 1,3,5-ТBB & 20 & 21.9 & 4.4 & 1.0 & 20.7 & 5.0 & 1.0 \\
\hline 1,3,5-ТCB & 20 & 22.3 & 2.5 & 0.5 & 18.7 & 23.2 & 4.3 \\
\hline 2,3,6-TCT & 20 & 20.9 & 3.4 & 0.7 & 20.3 & 5.8 & 1.2 \\
\hline $2,4,5-\mathrm{TCT}$ & 20 & 20.1 & 7.2 & 1.5 & 22.8 & 7.9 & 1.8 \\
\hline P5CB & 20 & 22.4 & 5.7 & 1.3 & 20.3 & 6.2 & 1.3 \\
\hline A2,6-TCT & 20 & 19.5 & 4.5 & 0.9 & 19.7 & 6.0 & 1.2 \\
\hline A-BHC & 20 & 20.2 & 6.9 & 1.4 & 18.0 & 5.8 & 1.0 \\
\hline G-BHC & 20 & 17.9 & 9.2 & 1.6 & 19.4 & 6.5 & 1.3 \\
\hline A-CHLA** & 20 & 20.3 & 2.5 & 0.5 & 36.4 & 11.8 & 4.3 \\
\hline Oxy-CHLA & 20 & 19.1 & 7.9 & 1.5 & 19.7 & 6.5 & 1.3 \\
\hline Aldrin & 20 & 21.4 & 2.1 & 0.5 & 19.5 & 6.5 & 1.3 \\
\hline Endrin & 20 & 17.8 & 13.7 & 2.5 & 12.0 & 24.6 & 2.9 \\
\hline Dieldrin & 20 & 18.1 & 9.8 & 1.8 & 20.0 & 12.9 & 2.6 \\
\hline Endos 1 & 20 & 17.2 & 11.0 & 1.9 & 19.3 & 10.8 & 2.1 \\
\hline Endos 2 & 20 & 15.9 & 11.1 & 1.8 & 18.5 & 4.7 & 0.9 \\
\hline Endos S & 20 & 16.6 & 8.7 & 1.4 & 18.5 & 4.7 & 0.9 \\
\hline Heptachlor & 20 & 15.8 & 8.2 & 1.3 & 22.1 & 6.8 & 1.5 \\
\hline OCSTYR & 20 & 20.0 & 7.9 & 1.6 & 19.5 & 6.2 & 1.2 \\
\hline Trans-Nonachlor & 20 & 18.7 & 7.5 & 1.4 & 18.4 & 6.5 & 1.2 \\
\hline o,p'-DDT & 20 & 16.9 & 3.2 & 0.5 & 22.2 & 5.1 & 1.1 \\
\hline p,p'-DDD & 20 & 15.9 & 8.4 & 1.3 & 18.5 & 3.8 & 0.7 \\
\hline p,p'-DDE & 20 & 18.8 & 3.3 & 0.6 & 18.2 & 4.7 & 0.9 \\
\hline p,p'-DDT & 20 & 18.6 & 8.8 & 1.6 & 19.6 & 5.4 & 1.1 \\
\hline Mirex & 20 & 18.1 & 7.7 & 1.4 & 18.5 & 2.3 & 0.4 \\
\hline p-Mirex $* * *$ & 40 & 18.4 & 8.6 & 1.6 & $\mathrm{n} / \mathrm{a}$ & $\mathrm{n} / \mathrm{a}$ & $\mathrm{n} / \mathrm{a}$ \\
\hline G-CHLA/PCB60* & 40 & 38.5 & 2.3 & 0.9 & 39.0 & 2.0 & 0.8 \\
\hline DMDT/PCB171 & 40 & 41.4 & 8.1 & 3.4 & 34.2 & 2.7 & 0.9 \\
\hline Cis-Nonachlor/PCB114 & 40 & 39.1 & 6.5 & 2.5 & $\mathrm{n} / \mathrm{a}$ & $\mathrm{n} / \mathrm{a}$ & $\mathrm{n} / \mathrm{a}$ \\
\hline H-Epoxide/PCB74 & 40 & 36.8 & 5.2 & 1.9 & $\mathrm{n} / \mathrm{a}$ & $\mathrm{n} / \mathrm{a}$ & $\mathrm{n} / \mathrm{a}$ \\
\hline H-Epoxide & 20 & $\mathrm{n} / \mathrm{a}$ & $\mathrm{n} / \mathrm{a}$ & $\mathrm{n} / \mathrm{a}$ & 20.8 & 11.5 & 2.4 \\
\hline Cis-Nonachlor & 20 & $\mathrm{n} / \mathrm{a}$ & $\mathrm{n} / \mathrm{a}$ & $\mathrm{n} / \mathrm{a}$ & 22.8 & 16.8 & 3.8 \\
\hline $\begin{array}{c}\text { PCB99/Alpha- } \\
\text { Chlordane }\end{array}$ & 40 & $\mathrm{n} / \mathrm{a}$ & $\mathrm{n} / \mathrm{a}$ & $\mathrm{n} / \mathrm{a}$ & 36.4 & 11.8 & 4.3 \\
\hline PCB167/Photo-Mirex & 40 & $\mathrm{n} / \mathrm{a}$ & $\mathrm{n} / \mathrm{a}$ & $\mathrm{n} / \mathrm{a}$ & 36.0 & 13.9 & 5.0 \\
\hline
\end{tabular}

*PCB74/H-epoxide coelutopn - $\mathrm{LN}_{2}$ modulator

**PCB99/alpha-chlordane coelution - UWmodulator

***PCB167/photo-Mirex coelution - UW modulator 
Table B.5 Reproducibility - Calculated concentration of $\mathrm{PCB} / \mathrm{OC} / \mathrm{CB}$ standards

\begin{tabular}{|c|c|c|c|c|}
\hline \multirow{2}{*}{ Name } & \multirow{2}{*}{$\begin{array}{c}\text { Design } \\
(\mathbf{n g} / \mathrm{mL})\end{array}$} & \multicolumn{3}{|c|}{$\begin{array}{l}\text { University of Waterloo } \\
\text { Modulator }(\mathrm{N}=5)\end{array}$} \\
\hline & & $\underset{(\mathbf{n g} / \mathbf{m L})}{\operatorname{Avg})}$ & $\begin{array}{c}\% \\
\text { RSD }\end{array}$ & $\begin{array}{c}\text { Std. } \\
\text { Deviation }\end{array}$ \\
\hline PCB4/PCB10 & 40 & 40.6 & 3.9 & 1.57 \\
\hline PCB6 & 20 & 20.2 & 6.1 & 1.23 \\
\hline PCB8 & 20 & 20.1 & 5.1 & 1.02 \\
\hline PCB15 & 20 & 21.0 & 7.1 & 1.50 \\
\hline PCB16 & 20 & 19.9 & 4.3 & 0.85 \\
\hline PCB18 & 20 & 21.0 & 4.7 & 1.00 \\
\hline PCB19 & 20 & 20.1 & 8.6 & 1.72 \\
\hline PCB22 & 20 & 19.0 & 4.8 & 0.91 \\
\hline PCB28/PCB31 & 40 & 38.6 & 1.6 & 0.63 \\
\hline PCB33 & 20 & 19.8 & 3.9 & 0.77 \\
\hline PCB37 & 20 & 19.1 & 3.6 & 0.69 \\
\hline PCB40 & 20 & 19.1 & 3.6 & 0.69 \\
\hline PCB41 & 20 & 19.2 & 5.0 & 0.97 \\
\hline PCB44 & 20 & 20.1 & 5.7 & 1.14 \\
\hline PCB49 & 20 & 18.9 & 3.5 & 0.65 \\
\hline PCB52 & 20 & 19.6 & 5.0 & 0.97 \\
\hline PCB54 & 20 & 20.2 & 5.2 & 1.05 \\
\hline PCB66 & 20 & 19.3 & 5.8 & 1.12 \\
\hline PCB70 & 20 & 18.8 & 10.8 & 2.04 \\
\hline PCB74* & 20 & 20.0 & 14.3 & 2.87 \\
\hline PCB77 & 20 & 19.2 & 7.3 & 1.40 \\
\hline PCB81 & 20 & 18.4 & 5.5 & 1.01 \\
\hline PCB84 & 20 & 19.3 & 4.9 & 0.94 \\
\hline PCB85 & 20 & 15.9 & 28.2 & 4.49 \\
\hline PCB87 & 20 & 18.5 & 4.8 & 0.89 \\
\hline PCB90/PCB101 & 40 & 37.4 & 3.2 & 1.19 \\
\hline PCB95 & 20 & 19.1 & 3.9 & 0.75 \\
\hline PCB97 & 20 & 18.6 & 4.2 & 0.79 \\
\hline PCB99** & 20 & 37.4 & 11.9 & 4.45 \\
\hline PCB104 & 20 & 18.7 & 15.0 & 2.80 \\
\hline PCB105 & 20 & 18.5 & 4.3 & 0.80 \\
\hline PCB110 & 20 & 19.4 & 5.1 & 1.00 \\
\hline PCB114 & 20 & 17.5 & 11.9 & 2.09 \\
\hline PCB118 & 20 & 19.8 & 5.0 & 1.00 \\
\hline PCB119 & 20 & 21.2 & 3.3 & 0.71 \\
\hline PCB123 & 20 & 20.5 & 13.1 & 2.68 \\
\hline PCB126 & 20 & 19.1 & 6.5 & 1.24 \\
\hline PCB128 & 20 & 18.2 & 4.9 & 0.89 \\
\hline
\end{tabular}


Table B.5 Reproducibility - Calculated concentration of $\mathrm{PCB} / \mathrm{OC} / \mathrm{CB}$ standards

\begin{tabular}{|c|c|c|c|c|}
\hline \multirow{2}{*}{ Name } & \multirow{2}{*}{$\begin{array}{l}\text { Design } \\
(\mathbf{n g} / \mathrm{mL})\end{array}$} & \multicolumn{3}{|c|}{$\begin{array}{l}\text { University of Waterloo } \\
\text { Modulator }(\mathbf{N}=\mathbf{5})\end{array}$} \\
\hline & & $\underset{(\mathbf{n g} / \mathbf{m L})}{\text { Avg. }}$ & $\begin{array}{c}\% \\
\text { RSD }\end{array}$ & $\begin{array}{c}\text { Std. } \\
\text { Deviation }\end{array}$ \\
\hline PCB129 & 20 & 18.1 & 4.4 & 0.79 \\
\hline PCB135 & 20 & 19.5 & 6.2 & 1.21 \\
\hline PCB137 & 20 & 18.5 & 4.9 & 0.90 \\
\hline PCB138 & 20 & 18.6 & 5.6 & 1.04 \\
\hline PCB141 & 20 & 18.7 & 3.9 & 0.73 \\
\hline PCB149 & 20 & 17.9 & 3.8 & 0.67 \\
\hline PCB151 & 20 & 20.8 & 7.9 & 1.64 \\
\hline PCB153 & 20 & 14.3 & 57.1 & 8.19 \\
\hline PCB155 & 20 & 19.4 & 3.9 & 0.76 \\
\hline PCB156 & 20 & 17.9 & 6.5 & 1.17 \\
\hline PCB157 & 20 & 18.8 & 6.4 & 1.20 \\
\hline PCB158 & 20 & 18.6 & 6.1 & 1.15 \\
\hline PCB167*** & 20 & $\mathrm{n} / \mathrm{a}$ & $\mathrm{n} / \mathrm{a}$ & $\mathrm{n} / \mathrm{a}$ \\
\hline PCB168 & 20 & 21.1 & 7.8 & 1.64 \\
\hline PCB169 & 20 & 17.5 & 6.9 & 1.21 \\
\hline PCB170 & 20 & 18.1 & 7.0 & 1.28 \\
\hline PCB174 & 20 & 18.5 & 5.0 & 0.93 \\
\hline PCB177 & 20 & 18.5 & 4.7 & 0.86 \\
\hline PCB178 & 20 & 19.7 & 4.9 & 0.97 \\
\hline PCB180 & 20 & 18.0 & 7.1 & 1.28 \\
\hline PCB183 & 20 & 18.8 & 6.8 & 1.28 \\
\hline PCB187 & 20 & 18.7 & 5.2 & 0.98 \\
\hline PCB188 & 20 & 18.9 & 4.0 & 0.75 \\
\hline PCB189 & 20 & 17.9 & 6.3 & 1.13 \\
\hline PCB191 & 20 & 18.7 & 11.6 & 2.16 \\
\hline PCB193 & 20 & 18.2 & 7.7 & 1.39 \\
\hline PCB194 & 20 & 18.2 & 5.3 & 0.96 \\
\hline PCB199 & 20 & 18.1 & 6.1 & 1.10 \\
\hline PCB200 & 20 & 19.3 & 6.4 & 1.24 \\
\hline PCB201 & 20 & 19.5 & 4.8 & 0.93 \\
\hline PCB202 & 20 & 18.3 & 7.2 & 1.32 \\
\hline PCB203 & 20 & 18.4 & 5.4 & 0.99 \\
\hline PCB205 & 20 & 18.5 & 5.0 & 0.92 \\
\hline PCB206 & 20 & 18.9 & 4.8 & 0.90 \\
\hline PCB207 & 20 & 18.3 & 4.4 & 0.81 \\
\hline PCB208 & 20 & 18.2 & 6.2 & 1.12 \\
\hline PCB209 & 20 & 17.8 & 5.0 & 0.89 \\
\hline HCB & 20 & 19.8 & 7.6 & 1.51 \\
\hline HCBD & 20 & $\mathrm{n} / \mathrm{a}$ & $\mathrm{n} / \mathrm{a}$ & $\mathrm{n} / \mathrm{a}$ \\
\hline
\end{tabular}


Table B.5 Reproducibility - Calculated concentration of $\mathrm{PCB} / \mathrm{OC} / \mathrm{CB}$ standards

\begin{tabular}{|c|c|c|c|c|}
\hline \multirow{2}{*}{ Name } & \multirow{2}{*}{$\begin{array}{c}\text { Design } \\
(\mathbf{n g} / \mathbf{m L})\end{array}$} & \multicolumn{3}{|c|}{$\begin{array}{l}\text { University of Waterloo } \\
\text { Modulator }(\mathrm{N}=5)\end{array}$} \\
\hline & & $\underset{(\mathbf{n g} / \mathbf{m L})}{\text { Avg. }}$ & $\begin{array}{c}\% \\
\text { RSD }\end{array}$ & $\begin{array}{c}\text { Std. } \\
\text { Deviation }\end{array}$ \\
\hline $\mathrm{HCE}$ & 20 & $\mathrm{n} / \mathrm{a}$ & $\mathrm{n} / \mathrm{a}$ & $\mathrm{n} / \mathrm{a}$ \\
\hline $1,2,3,4-\mathrm{TCB}$ & 20 & 21.1 & 7.2 & 1.51 \\
\hline $1,2,3,5-/ 1,2,4,5-\mathrm{TCB}$ & 40 & 39.6 & 8.3 & 3.28 \\
\hline $1,2,3-\mathrm{TCB}$ & 20 & 20.4 & 8.2 & 1.67 \\
\hline $1,2,4-\mathrm{TCB}$ & 20 & 19.0 & 5.0 & 0.94 \\
\hline $1,3,5-\mathrm{TBB}$ & 20 & 20.7 & 6.9 & 1.43 \\
\hline $1,3,5$-ТCB & 20 & 19.3 & 28.3 & 5.48 \\
\hline 2,3,6-TCT & 20 & 20.3 & 7.2 & 1.45 \\
\hline 2,4,5-TCT & 20 & 21.9 & 12.1 & 2.65 \\
\hline P5CB & 20 & 20.2 & 6.9 & 1.38 \\
\hline A2,6-TCT & 20 & 19.8 & 9.8 & 1.94 \\
\hline A-BHC & 20 & 18.1 & 8.8 & 1.59 \\
\hline G-BHC & 20 & 19.3 & 13.3 & 2.57 \\
\hline A-CHLA** & 20 & 37.4 & 11.9 & 4.45 \\
\hline Oxy-CHLA & 20 & 19.6 & 8.4 & 1.64 \\
\hline Aldrin & 20 & 19.3 & 10.0 & 1.94 \\
\hline Endrin & 20 & 14.3 & 33.4 & 4.79 \\
\hline Dieldrin & 20 & 22.6 & 9.1 & 2.05 \\
\hline Endos 1 & 20 & 16.5 & 18.9 & 3.12 \\
\hline Endos 2 & 20 & 18.4 & 11.4 & 2.09 \\
\hline Endos $\mathrm{S}$ & 20 & 18.4 & 11.4 & 2.09 \\
\hline Heptachlor & 20 & 20.8 & 51.7 & 10.73 \\
\hline OCSTYR & 20 & 19.3 & 9.0 & 1.73 \\
\hline Trans-Nonachlor & 20 & 18.3 & 10.8 & 1.99 \\
\hline o,p'-DDT & 20 & 23.8 & 34.9 & 8.31 \\
\hline p,p'-DDD & 20 & 18.6 & 11.6 & 2.16 \\
\hline p,p'-DDE & 20 & 18.5 & 5.5 & 1.01 \\
\hline p,p'-DDT & 20 & 20.4 & 44.8 & 9.15 \\
\hline Mirex & 20 & 18.0 & 6.1 & 1.10 \\
\hline p-Mirex*** & 40 & $\mathrm{n} / \mathrm{a}$ & $\mathrm{n} / \mathrm{a}$ & $\mathrm{n} / \mathrm{a}$ \\
\hline G-CHLA/PCB60* & 40 & 38.4 & 2.2 & 0.83 \\
\hline DMDT/PCB171 & 40 & 34.6 & 13.4 & 4.62 \\
\hline $\begin{array}{l}\text { Cis- } \\
\text { Nonachlor/PCB114 }\end{array}$ & 40 & $\mathrm{n} / \mathrm{a}$ & $\mathrm{n} / \mathrm{a}$ & $\mathrm{n} / \mathrm{a}$ \\
\hline H-Epoxide/PCB74 & 40 & $\mathrm{n} / \mathrm{a}$ & $\mathrm{n} / \mathrm{a}$ & $\mathrm{n} / \mathrm{a}$ \\
\hline H-Epoxide & 20 & 20.1 & 9.3 & 1.87 \\
\hline Cis-Nonachlor & 20 & 23.3 & 27.0 & 6.28 \\
\hline $\begin{array}{l}\text { PCB99/Alpha- } \\
\text { Chlordane }\end{array}$ & 40 & 37.4 & 11.9 & 4.45 \\
\hline PCB167/Photo-Mirex & 40 & 36.5 & 3.5 & 1.28 \\
\hline
\end{tabular}




\section{Appendix C}

The quantification of short-chain chlorinated paraffins in sediment samples using comprehensive two-dimensional gas chromatography with $\mu \mathrm{ECD}$ detection

Tables C.1 and C.2 - List of standards acquired from different providers

Table C.1 List of Standards - Dr. Ehrenstorfer

\begin{tabular}{|c|c|c|c|}
\hline $\begin{array}{c}\text { Chloroparaffin } \\
\text { Mix }\end{array}$ & $\% \mathrm{Cl}$ & $\begin{array}{c}\text { Concentration } \\
(\mathrm{ng} / \mu \mathrm{L})\end{array}$ & $\mathbf{P} / \mathbf{N}$ \\
\hline C10-13 & 55.5 & 100 & $\mathrm{X} 23105500 \mathrm{CY}$ \\
\hline C10-13 & 50.18 & 10 & LA11457512CY \\
\hline C10-13 & 51.5 & 100 & $\mathrm{X} 23105100 \mathrm{CY}$ \\
\hline C10-13 & 63 & 100 & X23106300 \\
\hline C14-17 & 42 & 100 & X23144200CY \\
\hline C14-17 & 52 & 100 & $\mathrm{X} 23145200 \mathrm{CY}$ \\
\hline C14-17 & 57 & 100 & X23145700CY \\
\hline $\mathrm{C} 10$ & 44.82 & 10 & LA11457510CY \\
\hline $\mathrm{C} 10$ & 50.18 & 10 & LA11457512CY \\
\hline $\mathrm{C} 10$ & 55 & 10.006 & LA11457514CY \\
\hline $\mathrm{C} 10$ & 60.09 & 10 & LA11457516CY \\
\hline $\mathrm{C} 10$ & 65.02 & 10 & LA11457518CY \\
\hline $\mathrm{C} 11$ & 45.5 & 10 & LA11457520CY \\
\hline $\mathrm{C} 11$ & 50.21 & 10 & LA11457522CY \\
\hline $\mathrm{C} 11$ & 55.2 & 10.007 & LA11457524CY \\
\hline $\mathrm{C} 11$ & 65.25 & 10.002 & LA11457528CY \\
\hline $\mathrm{C} 12$ & 45.32 & 10 & LA11457530CY \\
\hline $\mathrm{C} 12$ & 50.18 & 10 & LA11457532CY \\
\hline $\mathrm{C} 12$ & 55 & 10.002 & LA11457534CY \\
\hline $\mathrm{C} 12$ & 65.08 & 10 & LA11457536CY \\
\hline $\mathrm{C} 12$ & 69.98 & 10.004 & LA11457538CY \\
\hline $\mathrm{C} 13$ & 44.9 & 10 & LA11457540CY \\
\hline $\mathrm{C} 13$ & 50.23 & 10 & LA11457542CY \\
\hline $\mathrm{C} 13$ & 55.03 & 10 & LA11457544CY \\
\hline $\mathrm{C} 13$ & 59.98 & 10 & LA11457546CY \\
\hline $\mathrm{C} 13$ & 65.18 & 10 & LA11457548CY \\
\hline
\end{tabular}


Table C.2 List of Standards - Chiron

\begin{tabular}{|c|c|c|}
\hline Individual C10-C13 Chloroparaffin Standards & $\begin{array}{c}\text { Concentration } \\
(\mu \mathrm{g} / \mathrm{mL})\end{array}$ & $\mathbf{P} / \mathbf{N}$ \\
\hline 1,2-Dichlorodecane & 100 & 1666.10-K-IO \\
\hline 1,1,1,3-Tetrachlorodecane & 100 & $1662.10-\mathrm{K}-\mathrm{IO}$ \\
\hline 1,2,9,10-Tetrachlorodecane & 100 & 1671.10-K-IO \\
\hline $1,1,1,3,9,10-$-Hexachlorodecane (isomer mixture) & 100 & 1659.10-K-IO \\
\hline $1,1,1,3,8,10,10,10$-Octachlorodecane & 100 & $1622.10-\mathrm{K}-\mathrm{IO}$ \\
\hline 1,2-Dichloroundecane & 100 & 1667.11-K-IO \\
\hline 1,1,1,3-Tetrachloroundecane & 100 & 1649.11-K-IO \\
\hline 1,2,10,11-Tetrachloroundecane & 100 & 1674.11-K-IO \\
\hline $\begin{array}{l}1,1,1,3,10,11-\text { Hexachloroundecane (isomer } \\
\text { mixture) }\end{array}$ & 100 & 1650.11-K-IO \\
\hline 1,1,1,3,9,11,11,11-Octachloroundecane & 100 & 1623.11-K-IO \\
\hline 1,2-Dichlorododecane & 100 & 1668.12-K-IO \\
\hline 1,12-Dichlorododecane & 100 & 1663.12-K-IO \\
\hline 1,1,1,3-Tetrachlorododecane & 100 & 1651.12-K-IO \\
\hline 1,2,11,12-Tetrachlorododecane & 100 & $1675.12-\mathrm{K}-\mathrm{IO}$ \\
\hline $\begin{array}{l}\text { 1,1,1,3,11,12-Hexachlorododecane (isomer } \\
\text { mixture) }\end{array}$ & 100 & $1652.12-\mathrm{K}-\mathrm{IO}$ \\
\hline $1,1,1,3,10,12,12,12$-Octachlorododecane & 100 & 1624.12-K-IO \\
\hline 1,2-Dichlorotridecane & 100 & 1669.13-K-IO \\
\hline 1,1,1,3-Tetrachlorotridecane & 100 & 1653.13-K-IO \\
\hline 1,1,1,3,11,13,13,13-Octachlorotridecane & 100 & 1625.13-K-IO \\
\hline $\begin{array}{l}1,1,1,3,12,13 \text {-Hexachlorotridecane (isomer } \\
\text { mixture) }\end{array}$ & 100 & $1654.13-\mathrm{K}-\mathrm{IO}$ \\
\hline
\end{tabular}




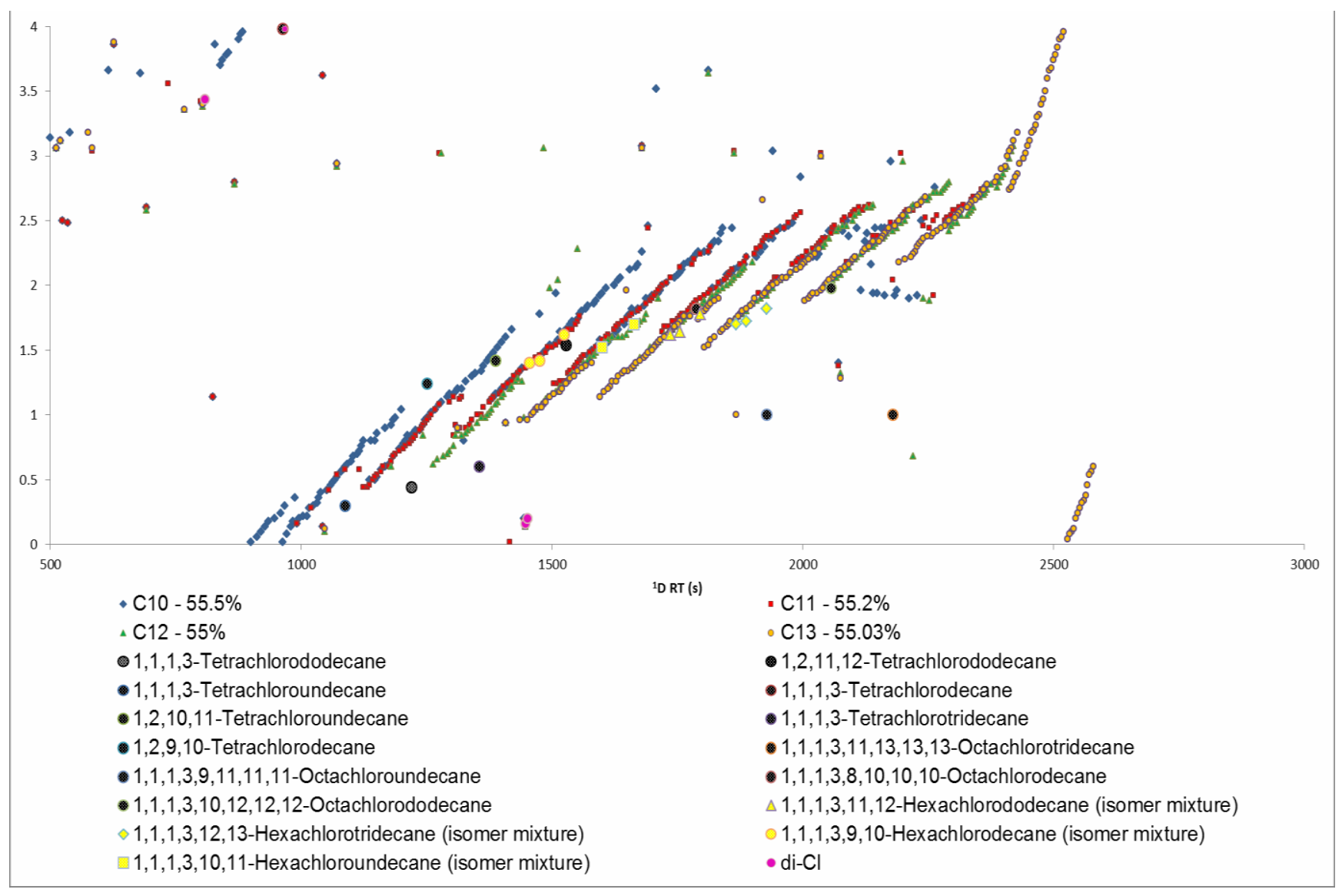

Fig. C.1 The overlaid retention times of individual $\mathrm{CP}$ standards and $\mathrm{C}_{10}, \mathrm{C}_{11}, \mathrm{C}_{12}$ and $\mathrm{C}_{13}$ standards with $55.5 \% \mathrm{Cl}$ 
Table C.3 Classifications: SCCP $55.5 \%$

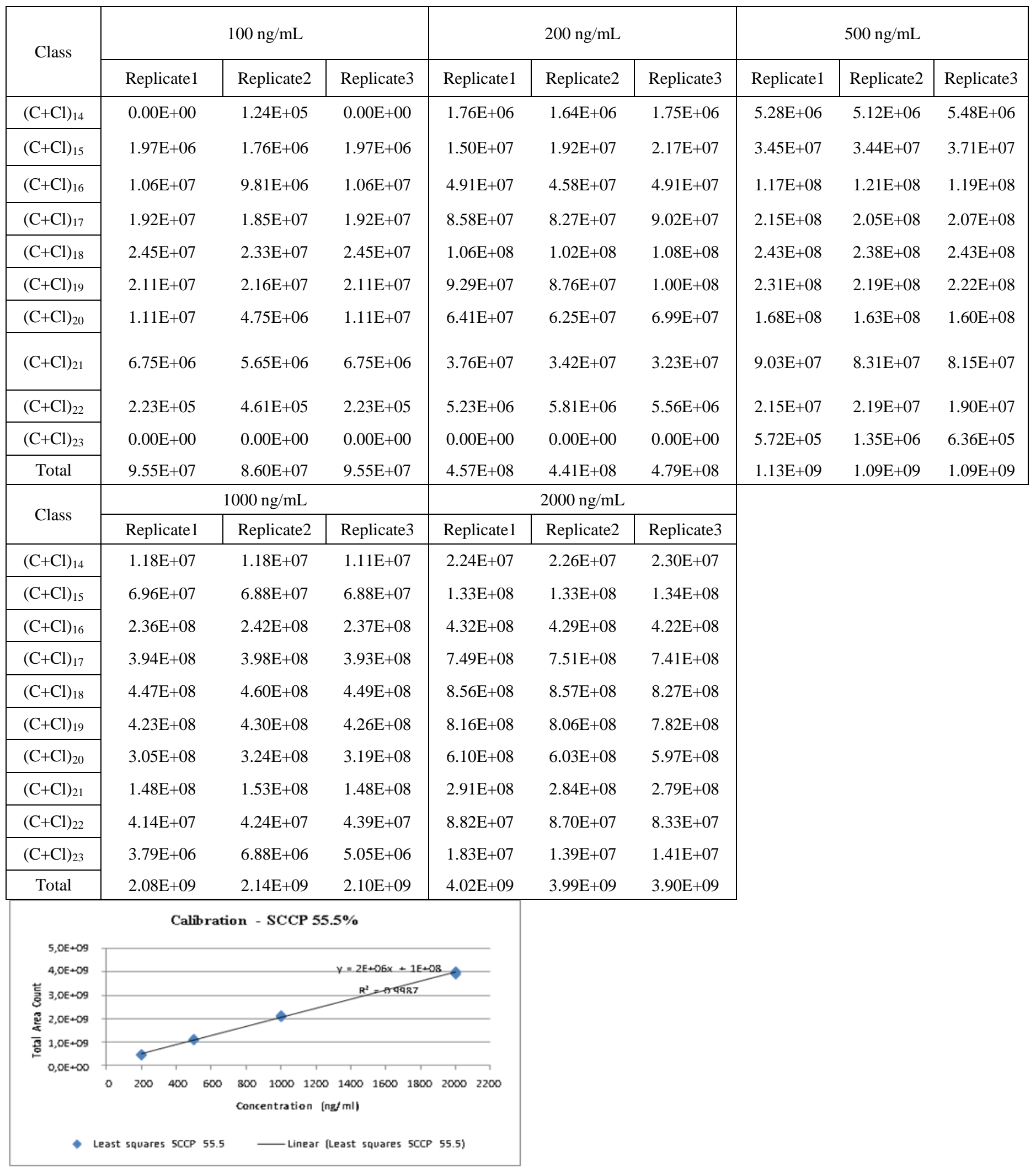




\section{Examples C.1 and C.2 - Example of SCCPs identification and quantitation in sediments}

\section{Example C.1: Quasimeme Phase III - Cleaned-up extract of sediment sample}

Step1: Visually inspect the Classifications patterns in ChromaTOF and confirm the retention times of the least retained peak in each classification. The sample best resembles the SCCP $63 \% \mathrm{Cl}$ pattern.

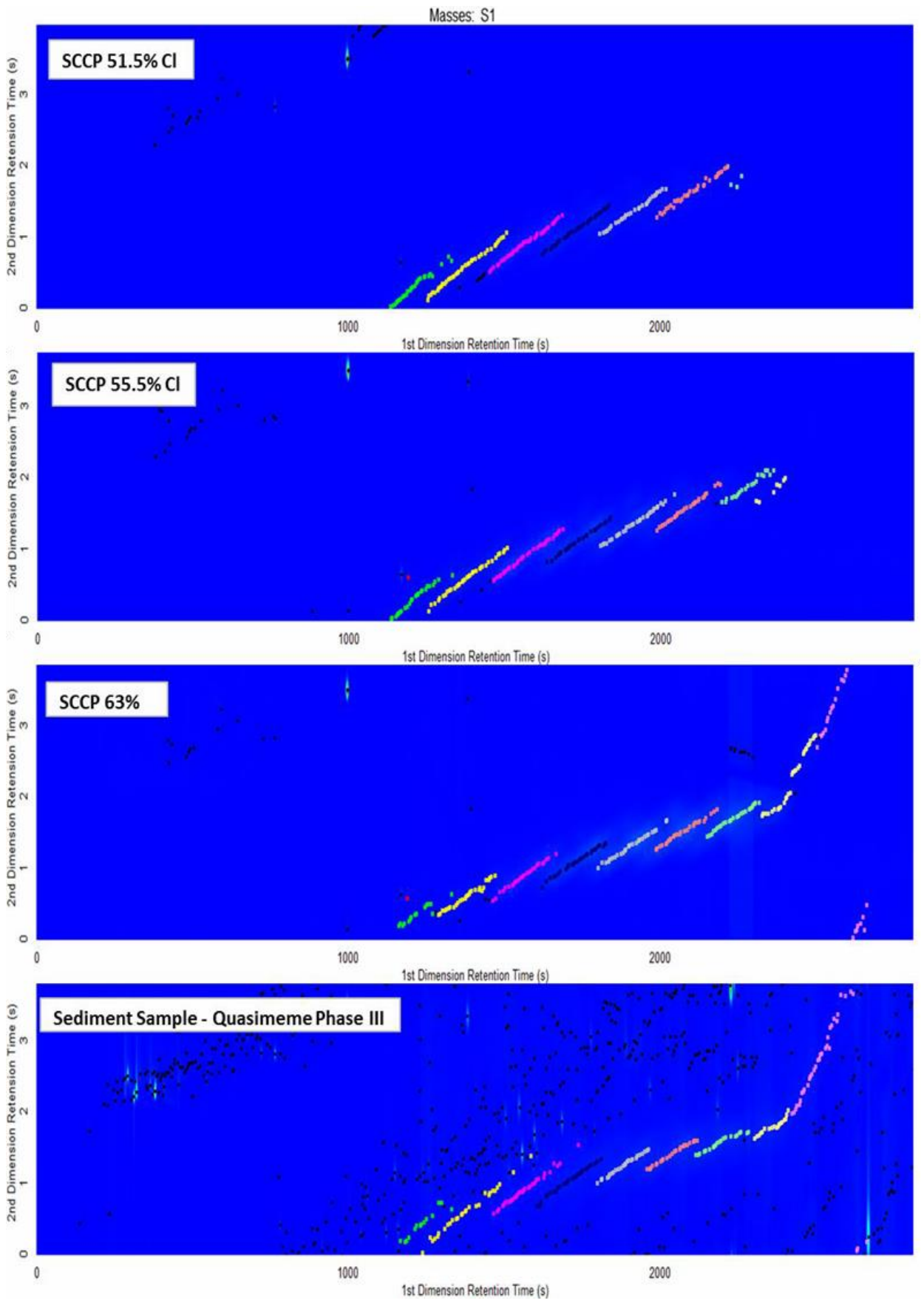


Step 2: Compare areas and area \% generated by the classification function $( \pm 20 \%)$ to find the best match to the standard mix for quantification purposes. The sample best matches the SCCP 63 area \% generated through ChromaTOF Classification function.

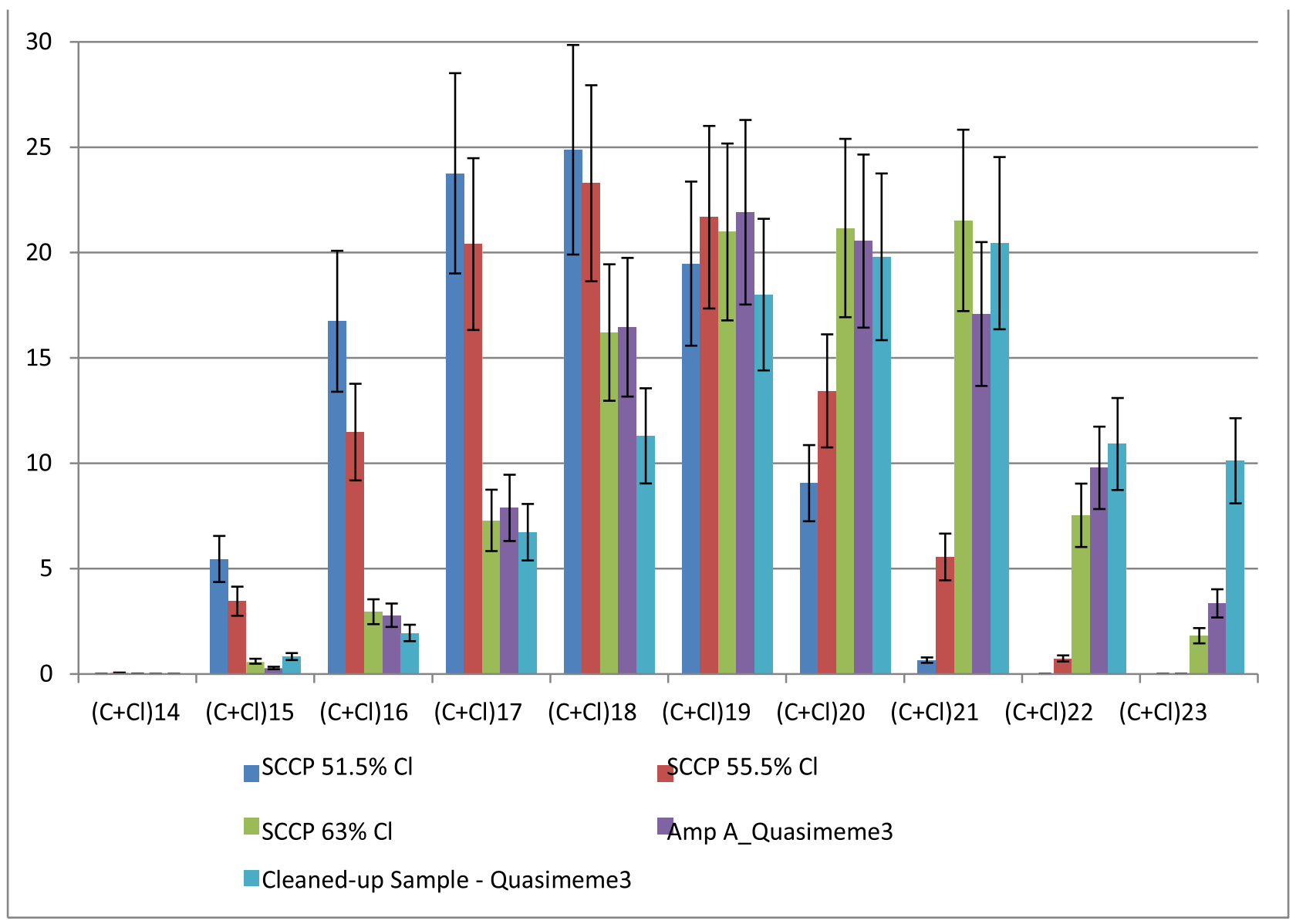

Step 3: Export the summary classifications (area) to Excel and apply the calibration curve of the standard that best resembles the pattern in the sample.

Based on the information obtained in Steps 1 and 2, the sample will be quantitated using the SCCP $63 \% \mathrm{Cl}$ multi-level calibration (see Table S3.3 - data presented for SCCP $55.5 \% \mathrm{Cl}$ ). 


\section{Example C.2: Sediment Sample Collected in Ontario}

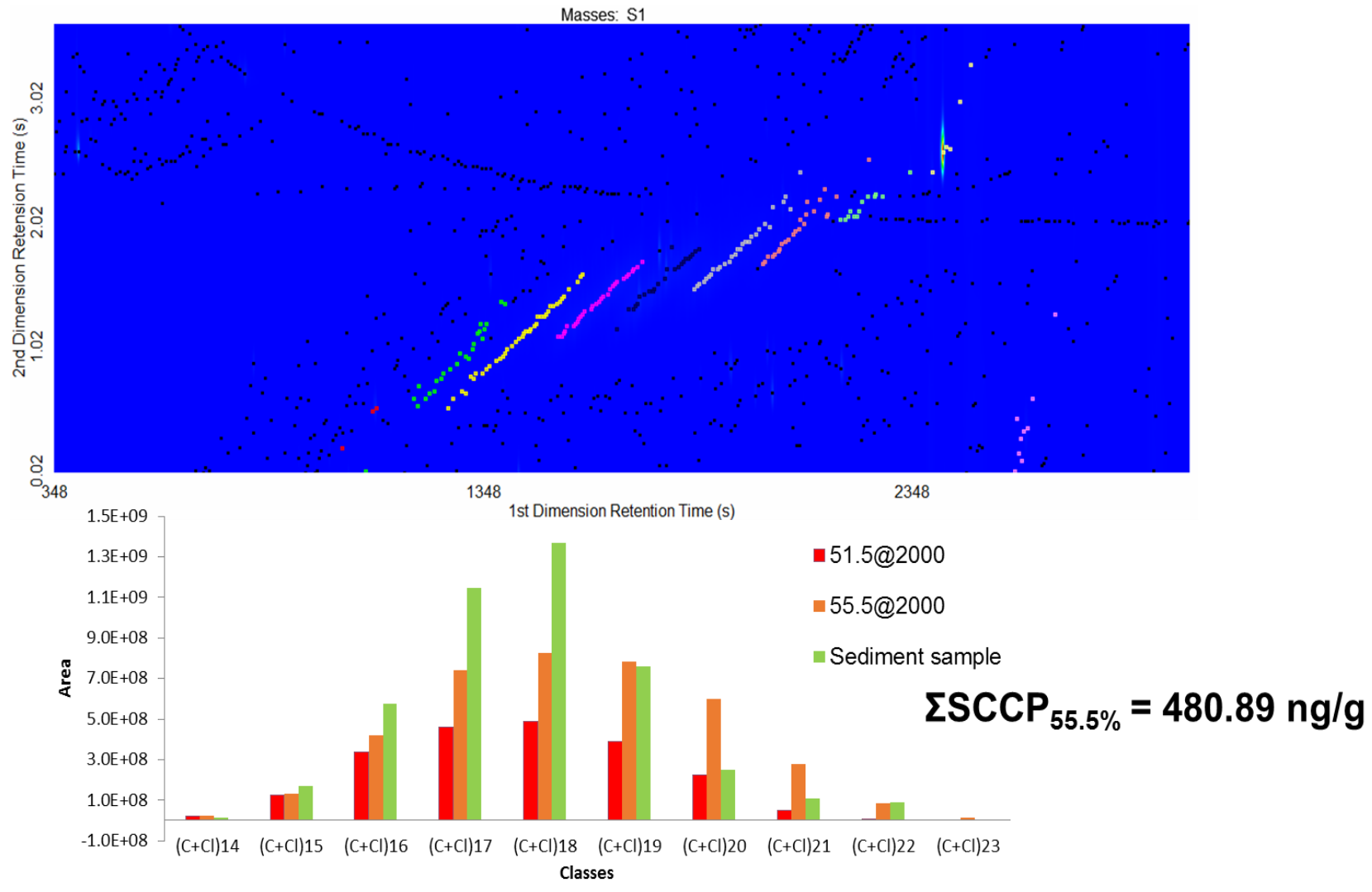




\section{Appendix D}

The Analysis of Polychlorinated Diphenyl Ethers in Sediments by Comprehensive Two-Dimensional Gas Chromatography with Micro-electron Capture Detection and Time-of-flight Mass Spectrometry

Examples of Mass Spectra and Results

Examples of mass spectra and confirmation for mono- to trichlorinated PCDEs - high levels, not previously reported

\section{VB16-06A vs. PCDE Sediment Spike}

*PCDE-di-1/PCDE12 (bottom mass spectrum - labelled PCDE 12)
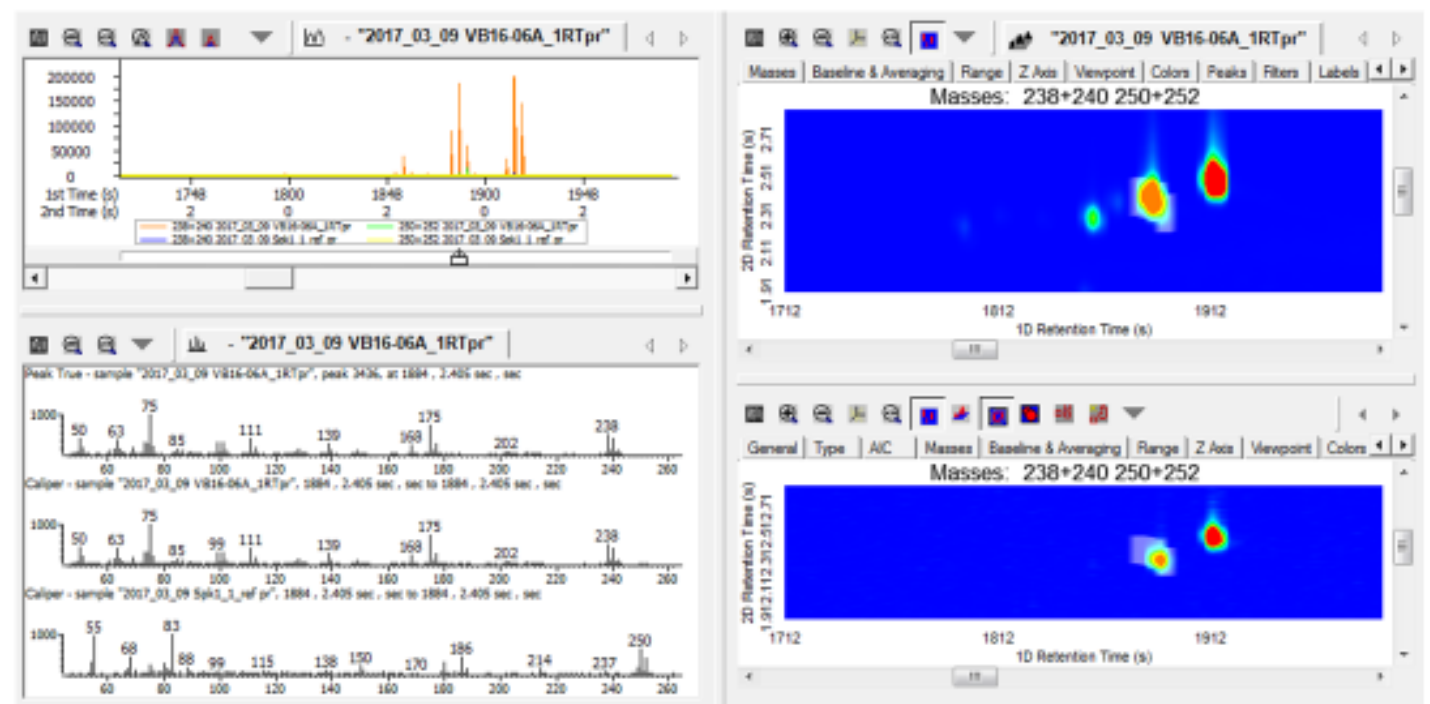


\section{VB16-06A vs. PCDE Sediment Spike}

PCDE-3 (4-monoCl) m/z 204+206 (m/z 216+218 labelled PCDE-3)
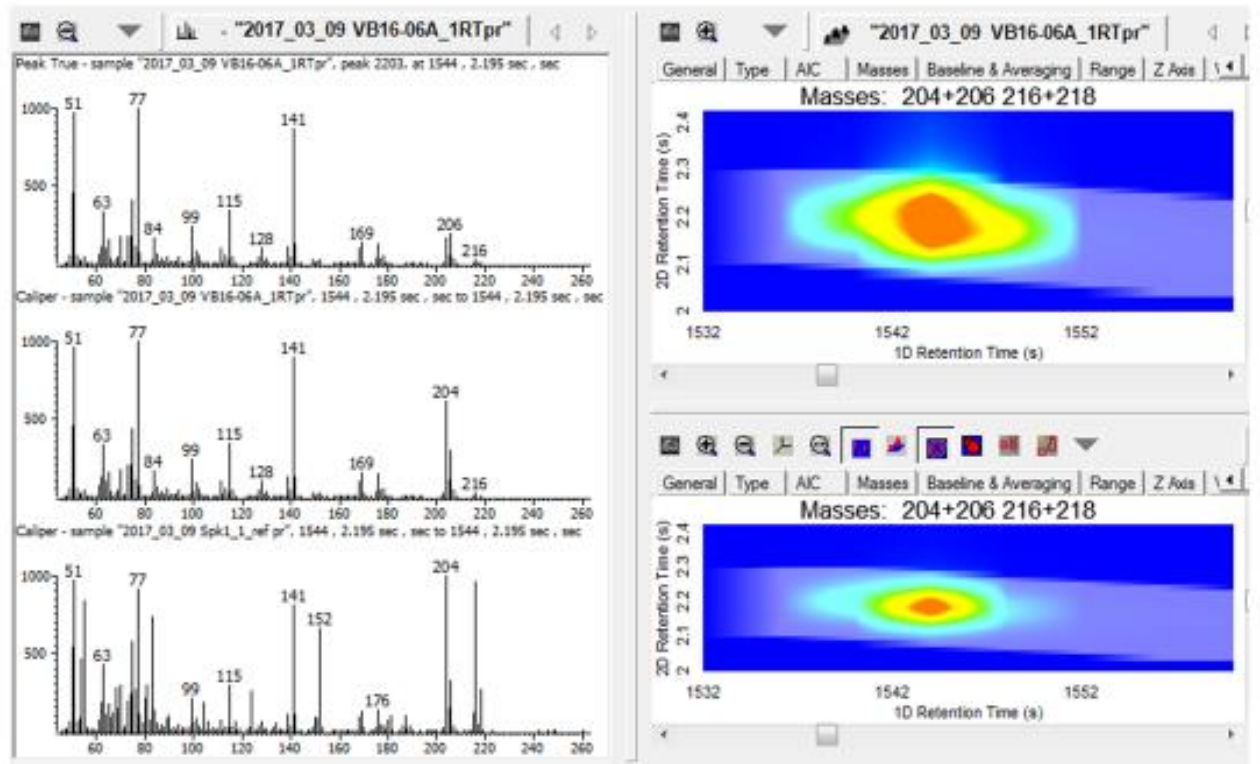

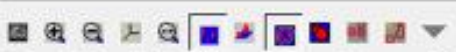

General | Type | AC | Masses | Bascine s Averoging | Range | 2 how | If I

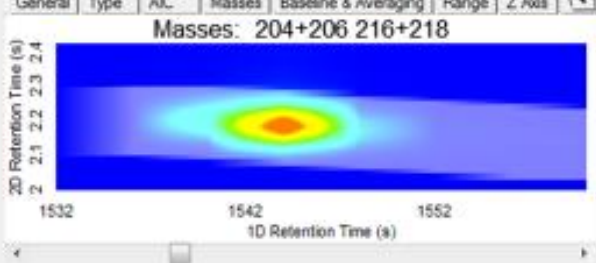

\section{VB16-06A vs. PCDE Sediment Spike}

PCDE-28 (2,4,4'-triCl)
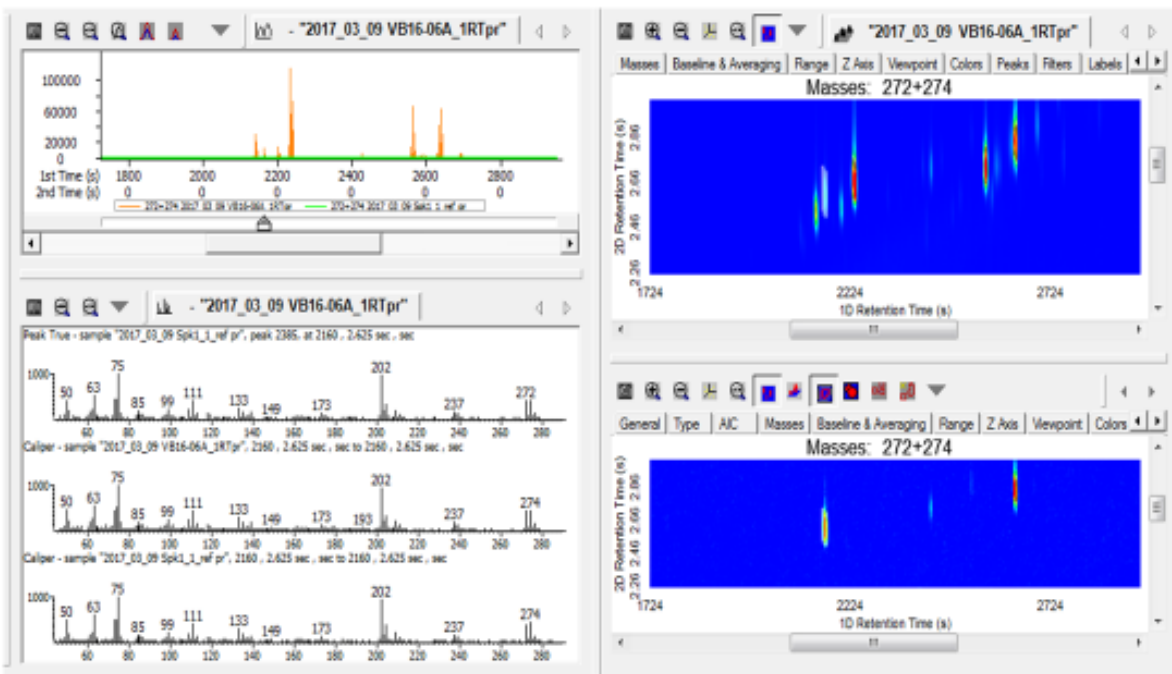
Table D.1 - PCDE results reported for all 37 samples analyzed:

\begin{tabular}{|c|c|c|c|c|c|c|c|c|c|c|c|c|c|c|c|c|}
\hline Name & \begin{tabular}{|l|} 
VB16- \\
01A \\
\end{tabular} & $\begin{array}{l}\text { VB16- } \\
\text { 01B }\end{array}$ & $\begin{array}{l}\text { VB16- } \\
\text { 01C }\end{array}$ & $\begin{array}{l}\text { VB16- } \\
\text { 02A }\end{array}$ & $\begin{array}{l}\text { VB16- } \\
\text { 02B }\end{array}$ & $\begin{array}{l}\text { VB16- } \\
\text { 02C }\end{array}$ & $\begin{array}{l}\text { VB16- } \\
\text { 04A }\end{array}$ & $\begin{array}{l}\text { VB16- } \\
\text { 04B }\end{array}$ & $\begin{array}{l}\text { VB16- } \\
\text { 04C }\end{array}$ & $\begin{array}{l}\text { VB16- } \\
\text { 05A }\end{array}$ & $\begin{array}{l}\text { VB16- } \\
\text { 05B }\end{array}$ & $\begin{array}{l}\text { VB16- } \\
\text { 05C }\end{array}$ & $\begin{array}{l}\text { VB16- } \\
\text { 06A }\end{array}$ & $\begin{array}{l}\text { VB16- } \\
\text { 06B }\end{array}$ & $\begin{array}{l}\text { VB16- } \\
\text { 06C }\end{array}$ & $\begin{array}{l}\text { VB16- } \\
\text { 06D }\end{array}$ \\
\hline PCDE-3 (4-monoCl) & 10.6 & 63.5 & 37.0 & 13.2 & 4.0 & 0.7 & 1.9 & 0.5 & & 17.7 & 0.5 & & 1120.1 & 25.7 & 2.8 & 4.5 \\
\hline *PCDE-mono-1 & 14.9 & 17.4 & 10.7 & 9.4 & 2.4 & 0.6 & 1.1 & & & 12.4 & & & 182.1 & 7.1 & 0.8 & 0.9 \\
\hline *PCDE-mono-2 & 1.0 & 1.4 & 0.5 & 0.8 & & & & & & 0.6 & & & 12.4 & 0.8 & & \\
\hline PCDE-15 (4,4'-diCl) & 53.1 & 172.9 & 38.9 & 20.7 & 5.5 & 2.4 & 3.7 & 2.2 & & 23.9 & 2.4 & & 918.5 & 12.2 & 3.7 & 3.9 \\
\hline *PCDE-di-1/PCDE12 & 39.2 & 62.3 & 72.0 & 23.7 & 7.7 & 2.4 & 3.0 & & & 18.3 & 2.4 & & 859.6 & 12.5 & 3.5 & 2.6 \\
\hline *PCDE-di-2 & 8.3 & 12.2 & 2.9 & 2.6 & 2.8 & 2.3 & & & & 6.3 & & & 15.6 & 2.2 & & \\
\hline *PCDE-di-3 & 3.4 & 7.5 & 3.7 & 2.2 & & & & & & 3.2 & & & 7.4 & 2.3 & & \\
\hline *PCDE-di-4 & 2.2 & & 2.8 & & & & & & & & & & & & & \\
\hline *PCDE-di-5 & & & & & & & & & & & & & 4.8 & & & \\
\hline *PCDE-di-6 & & & & & & & & & & & & & 4.7 & & & \\
\hline *PCDE-di-7 & 9.1 & 5.0 & 6.9 & 6.9 & 3.7 & & & & & 4.4 & & & 73.0 & 3.8 & 2.4 & \\
\hline PCDE-28 $(2,4,4$ '-triCl) & 7.3 & 11.9 & 3.6 & 3.6 & 2.7 & & & & & 3.2 & & & 55.0 & 2.7 & & \\
\hline *PCDE-37-tri & 14.9 & 70.1 & 38.9 & 8.1 & 3.8 & & 2.6 & & & 6.4 & & & 405.2 & 5.1 & 2.3 & 2.7 \\
\hline *PCDE-tri-1 & 50.4 & 65.4 & 5.8 & & 5.6 & 2.5 & 2.7 & & & 14.3 & & & 146.5 & 3.6 & 2.4 & \\
\hline *PCDE-tri-2 & 6.3 & 6.0 & 3.2 & & 2.7 & & & & & 2.9 & & & 11.9 & & & \\
\hline *PCDE-tri-3 & 3.5 & 6.0 & 10.9 & & 2.6 & & & & & 2.5 & & & 29.0 & 2.4 & & \\
\hline *PCDE-tri-4 & 2.2 & & & & & & & & & & & & & & & \\
\hline *PCDE-tri-5 & & & & & & & & & & & & & 5.1 & & & \\
\hline *PCDE-tri-6 & & 3.4 & & & & & & & & & & & 3.9 & & & \\
\hline \multicolumn{17}{|l|}{ *PCDE-tri-7 } \\
\hline $\begin{array}{l}\text { PCDE-74 }(2,4,4 ', 5- \\
\text { TetraCl) }\end{array}$ & 52.3 & 104.2 & 23.7 & 19.5 & 6.7 & & 3.1 & & & 11.5 & & & 258.6 & 5.3 & 2.8 & 2.7 \\
\hline $\begin{array}{l}\text { PCDE-77 (3,3',4,4'- } \\
\text { TetraCl) }\end{array}$ & 10.4 & 19.5 & 19.3 & 6.8 & 4.4 & & & & & 4.7 & & & 89.4 & 3.9 & & \\
\hline *PCDE-tetra-1 & 14.1 & 24.1 & 6.7 & 6.9 & 3.6 & & 2.8 & & & 5.2 & & & 95.5 & 3.9 & & 2.7 \\
\hline *PCDE-tetra-2 & 10.2 & 14.9 & 4.2 & 3.7 & & & & & & 3.3 & & & 26.5 & 2.7 & & \\
\hline *PCDE-tetra-3 & 5.9 & 3.6 & & 3.5 & & & & & & 2.8 & & & 3.8 & & & \\
\hline *PCDE-tetra-4 & 3.6 & 4.0 & & 2.8 & & & & & & & & & 9.8 & & & \\
\hline *PCDE-tetra-5 & 6.8 & 12.3 & 5.8 & 3.6 & & & & & & & & & 12.2 & & & \\
\hline *PCDE-tetra-6 & 5.1 & 12.2 & 4.6 & 3.6 & & & & & & 4.4 & & & 30.0 & 3.0 & & \\
\hline$*$ PCDE-tetra-7 & & & & & & & & & & & & & 7.6 & & & \\
\hline \multicolumn{17}{|l|}{ *PCDE-tetra-8 } \\
\hline $\begin{array}{l}\text { PCDE-99 }\left(2,2^{\prime}, 4,4^{\prime}, 5-\right. \\
\text { PentaCl })\end{array}$ & 90.7 & 165.8 & 52.0 & 38.6 & 13.5 & & 4.3 & & & 17.3 & & & 375.7 & 6.5 & 3.1 & \\
\hline *PCDE-penta-1 & 5.0 & 8.3 & 4.4 & 3.7 & & & & & & & & & 14.2 & & & \\
\hline *PCDE-penta-2 & 70.5 & 100.0 & 23.1 & 24.4 & 8.9 & & 3.6 & 2.9 & & 11.8 & & & 375.8 & 6.3 & 3.2 & 3.4 \\
\hline
\end{tabular}




\begin{tabular}{|c|c|c|c|c|c|c|c|c|c|c|c|c|c|c|c|c|}
\hline Name & $\begin{array}{l}\text { VB16- } \\
\text { 01A }\end{array}$ & $\begin{array}{l}\text { VB16- } \\
\text { 01B }\end{array}$ & $\begin{array}{l}\text { VB16- } \\
\text { 01C }\end{array}$ & $\begin{array}{l}\text { VB16- } \\
\text { 02A }\end{array}$ & $\begin{array}{l}\text { VB16- } \\
\text { 02B }\end{array}$ & $\begin{array}{l}\text { VB16- } \\
\text { 02C }\end{array}$ & $\begin{array}{l}\text { VB16- } \\
\text { 04A }\end{array}$ & $\begin{array}{l}\text { VB16- } \\
\text { 04B }\end{array}$ & $\begin{array}{l}\text { VB16- } \\
\text { 04C }\end{array}$ & $\begin{array}{l}\text { VB16- } \\
\text { 05A }\end{array}$ & $\begin{array}{l}\text { VB16- } \\
\text { 05B }\end{array}$ & $\begin{array}{l}\text { VB16- } \\
\text { 05C }\end{array}$ & $\begin{array}{l}\text { VB16- } \\
\text { 06A }\end{array}$ & $\begin{array}{l}\text { VB16- } \\
\text { 06B }\end{array}$ & $\begin{array}{l}\text { VB16- } \\
\text { 06C }\end{array}$ & $\begin{array}{l}\text { VB16- } \\
\text { 06D }\end{array}$ \\
\hline $\begin{array}{l}\text { *PCDE-penta- } \\
\text { 3_PCDE86 }\end{array}$ & 11.8 & 21.1 & 8.7 & 6.4 & 3.9 & & & & & 3.4 & & & 27.2 & & & \\
\hline *PCDE-penta-4 & 10.7 & 15.4 & 7.8 & 4.9 & 3.7 & & & & & 3.4 & & & 8.7 & 3.0 & & \\
\hline *PCDE-penta-5 & 5.0 & 5.8 & & 3.0 & & & & & & & & & 5.9 & & & \\
\hline *PCDE-penta-6 & 5.7 & 9.4 & 4.1 & 3.8 & & & & & & & & & 19.7 & & & \\
\hline *PCDE-penta-7 & 6.1 & 8.3 & & 4.0 & & & & & & & & & 11.6 & & & \\
\hline *PCDE-penta-8 & 7.0 & 11.0 & 5.3 & 4.4 & & & & & & 3.5 & & & 22.1 & 3.0 & & \\
\hline *PCDE-penta-9 & & & & & & & & & & & & & 4.9 & & & \\
\hline *PCDE-penta-10 & & & & & & & & & & & & & 6.3 & & & \\
\hline *PCDE-penta-11 & & & & & & & & & & & & & 2.9 & & & \\
\hline \multicolumn{17}{|l|}{ *PCDE-penta-12 } \\
\hline *PCDE141 & 26.2 & 45.2 & 15.1 & 7.1 & 3.9 & & & & & 3.6 & & & 43.8 & 3.2 & & \\
\hline *PCDE-hexa-1 & 134.5 & 257.3 & 57.5 & 31.6 & 13.8 & & 3.7 & & & 17.3 & & & 619.7 & 11.3 & 3.8 & 3.8 \\
\hline *PCDE-hexa-2 & 33.3 & 55.0 & 13.2 & 8.8 & 5.1 & & & & & 5.1 & & & 199.8 & 4.6 & 2.9 & 2.9 \\
\hline *PCDE-hexa-3 & 13.0 & 27.8 & 7.0 & 4.9 & 3.7 & & & & & 3.8 & & & 44.5 & 3.1 & & \\
\hline *PCDE-hexa-4 & 123.7 & 251.5 & 51.1 & 27.2 & 13.2 & & 2.9 & & & 14.4 & & & 440.3 & 6.4 & & 3.1 \\
\hline *PCDE-hexa-5 & 64.5 & 94.9 & 35.2 & 20.6 & 7.7 & & & & & 8.6 & & & 93.8 & 4.7 & & \\
\hline *PCDE-hexa-6 & 4.1 & 16.9 & 7.0 & 4.2 & & & & & & & & & 16.2 & & & \\
\hline *PCDE-hexa-7 & 34.2 & 65.6 & 22.3 & 11.5 & 5.4 & & & & & 4.3 & & & 59.8 & 3.4 & & \\
\hline *PCDE-hexa-8 & 4.8 & 6.2 & 4.1 & 3.9 & & & & & & & & & 4.7 & & & \\
\hline *PCDE-hexa-9 & & & & & & & & & & & & & 3.3 & & & \\
\hline \multicolumn{17}{|l|}{ *PCDE-hexa-10 } \\
\hline $\begin{array}{l}\text { PCDE-180 } \\
\left(2,2^{\prime}, 3,4,44^{\prime}, 5,5^{\prime}-\right. \\
\text { HeptaCl })\end{array}$ & 34.7 & 29.2 & 12.2 & 7.4 & 3.5 & & & & & & & & 78.7 & 2.5 & & \\
\hline *PCDE-hepta-1 & 252.2 & 251.4 & 95.7 & 46.9 & 13.8 & & & & & 17.4 & & & 466.4 & 7.8 & & \\
\hline *PCDE-hepta-2 & 112.7 & 120.1 & 42.1 & 22.0 & 5.6 & & & & & 6.8 & & & 260.8 & 4.2 & 2.2 & \\
\hline *PCDE-hepta-3 & 8.8 & 12.7 & 5.6 & 3.6 & & & & & & 2.5 & & & 28.2 & & & \\
\hline *PCDE-hepta-4 & 74.6 & 80.3 & 31.4 & 12.0 & 4.7 & & & & & 5.4 & & & 179.4 & 3.4 & & \\
\hline *PCDE-hepta-5 & & 2.9 & & & & & & & & & & & 8.0 & & & \\
\hline *PCDE-hepta-6 & & & & & & & & & & & & & 17.2 & & & \\
\hline *PCDE-hepta-7 & & & & & & & & & & & & & 3.7 & & & \\
\hline *PCDE-octa-1 & 41.4 & 35.9 & 12.5 & 7.4 & 3.0 & & & & & 4.4 & & & 123.2 & 2.7 & & \\
\hline *PCDE-octa-2 & 7.2 & 6.4 & 2.2 & & & & & & & & & & 26.8 & & & \\
\hline *PCDE-octa-3 & 2.4 & 5.1 & 3.1 & & & & & & & & & & 14.3 & & & \\
\hline *PCDE-octa-4 & & & & & & & & & & & & & 10.9 & & & \\
\hline *PCDE-octa-5 & & & & & & & & & & & & & & & & \\
\hline
\end{tabular}




\begin{tabular}{|c|c|c|c|c|c|c|c|c|c|c|c|c|c|c|c|c|}
\hline Name & $\begin{array}{c}\text { VB16- } \\
\text { 01A }\end{array}$ & $\begin{array}{c}\text { VB16- } \\
\text { 01B } \\
\end{array}$ & $\begin{array}{c}\text { VB16- } \\
\text { 01C }\end{array}$ & $\begin{array}{c}\text { VB16- } \\
\text { 02A }\end{array}$ & $\begin{array}{c}\text { VB16- } \\
\text { 02B } \\
\end{array}$ & $\begin{array}{c}\text { VB16- } \\
\text { 02C } \\
\end{array}$ & $\begin{array}{c}\text { VB16- } \\
\text { 04A }\end{array}$ & $\begin{array}{c}\text { VB16- } \\
\text { 04B } \\
\end{array}$ & $\begin{array}{c}\text { VB16- } \\
\text { 04C } \\
\end{array}$ & $\begin{array}{c}\text { VB16- } \\
\text { 05A }\end{array}$ & $\begin{array}{c}\text { VB16- } \\
\text { 05B }\end{array}$ & $\begin{array}{c}\text { VB16- } \\
\text { 05C }\end{array}$ & $\begin{array}{c}\text { VB16- } \\
\text { 06A }\end{array}$ & $\begin{array}{c}\text { VB16- } \\
\text { 06B }\end{array}$ & $\begin{array}{c}\text { VB16- } \\
\text { 06C }\end{array}$ & $\begin{array}{c}\text { VB16 } \\
\text { 06D }\end{array}$ \\
\hline *PCDE-nona-1 & & & & & & & & & & & & & 11.3 & & & \\
\hline
\end{tabular}

Note: samples are reported as $\mathbf{n g} / \mathbf{g}$

Note: Diluted results reported for -06A for mono- and di-Cl-PCDEs

\begin{tabular}{|c|c|c|c|c|c|c|c|c|c|c|c|c|c|c|c|c|}
\hline $\begin{array}{l}\text { Total PCDE } \\
\text { Calculations } \\
\end{array}$ & $\begin{array}{c}\text { VB16- } \\
\text { 01A }\end{array}$ & $\begin{array}{c}\text { VB16- } \\
\text { 01B }\end{array}$ & $\begin{array}{l}\text { VB16- } \\
\text { 01C }\end{array}$ & $\begin{array}{l}\text { VB16- } \\
\text { 02A }\end{array}$ & $\begin{array}{l}\text { VB16- } \\
\text { 02B }\end{array}$ & $\begin{array}{l}\text { VB16- } \\
\text { 02C }\end{array}$ & $\begin{array}{l}\text { VB16- } \\
\text { 04A }\end{array}$ & $\begin{array}{l}\text { VB16- } \\
\text { 04B }\end{array}$ & $\begin{array}{c}\text { VB- } \\
16-4 C\end{array}$ & $\begin{array}{c}\text { VB16- } \\
\text { 05A }\end{array}$ & $\begin{array}{l}\text { VB16- } \\
\text { 05B }\end{array}$ & $\begin{array}{c}\text { VB- } \\
16-5 C\end{array}$ & $\begin{array}{l}\text { VB16- } \\
\text { 06A }\end{array}$ & $\begin{array}{l}\text { VB-16- } \\
\text { 06B }\end{array}$ & $\begin{array}{l}\text { VB-16- } \\
\text { 06C }\end{array}$ & $\begin{array}{l}\text { VB16- } \\
\text { 06D }\end{array}$ \\
\hline $\begin{array}{l}\text { Total mono-Cl- } \\
\text { PCDE }\end{array}$ & 26.5 & 82.3 & 48.2 & 23.4 & 6.4 & 1.3 & 3.1 & 0.5 & & 30.6 & 0.5 & & 1314.5 & 33.5 & 3.6 & 5.4 \\
\hline Total di-Cl-PCDE & 115.3 & 259.8 & 127.2 & 56.2 & 19.8 & 7.1 & 6.8 & 2.2 & & 56.2 & 4.9 & & 1883.5 & 32.9 & 9.6 & 6.6 \\
\hline Total tri-Cl-PCDE & 84.6 & 162.7 & 62.4 & 11.7 & 17.4 & 2.5 & 5.3 & & & 29.3 & & & 656.6 & 13.7 & 4.6 & 2.7 \\
\hline Total tetra-Cl-PCDE & 108.4 & 194.8 & 64.3 & 50.3 & 14.8 & & 5.9 & & & 31.8 & & & 533.3 & 18.8 & 2.8 & 5.4 \\
\hline $\begin{array}{l}\text { Total penta-Cl- } \\
\text { PCDE }\end{array}$ & 212.3 & 344.9 & 105.4 & 93.1 & 29.9 & & 7.9 & 2.9 & & 39.3 & & & 875.1 & 18.7 & 6.3 & 3.4 \\
\hline Total hexa-Cl-PCDE & 438.3 & 820.2 & 212.4 & 119.6 & 52.9 & & 6.6 & & & 57.1 & & & 1526.0 & 36.6 & 6.7 & 9.9 \\
\hline $\begin{array}{l}\text { Total hepta-Cl- } \\
\text { PCDE }\end{array}$ & 482.9 & 496.7 & 187.0 & 91.9 & 27.6 & & & & & 32.1 & & & 1042.3 & 17.9 & 2.2 & \\
\hline Total octa-Cl-PCDE & 51.0 & 47.3 & 17.8 & 7.4 & 3.0 & & & & & 4.4 & & & 175.2 & 2.7 & & \\
\hline Total nona-Cl-PCDE & & & & & & & & & & & & & 11.3 & & & \\
\hline Total PCDE (ng/g) & 1519.3 & 2408.6 & 824.6 & 453.6 & 171.8 & 10.9 & 35.6 & 5.5 & ND & 280.7 & 5.3 & ND & 8017.8 & 174.8 & 35.8 & 33.3 \\
\hline
\end{tabular}

ND $=$ not detected 


\begin{tabular}{|c|c|c|c|c|c|c|c|c|c|c|c|c|c|c|}
\hline Name & $\begin{array}{l}\text { VB16- } \\
\text { 07A }\end{array}$ & $\begin{array}{l}\text { VB16- } \\
\text { 07B }\end{array}$ & $\begin{array}{l}\text { VB16- } \\
\text { 07C }\end{array}$ & $\begin{array}{l}\text { VB16- } \\
\text { 07D }\end{array}$ & $\begin{array}{l}\text { VB16- } \\
\text { 08A }\end{array}$ & $\begin{array}{l}\text { VB16- } \\
\text { 08B }\end{array}$ & $\begin{array}{l}\text { VB16- } \\
\text { 08C }\end{array}$ & $\begin{array}{l}\text { VB16- } \\
\text { 08D }\end{array}$ & $\begin{array}{l}\text { VB16- } \\
\text { 09A }\end{array}$ & $\begin{array}{l}\text { VB16- } \\
\text { 09B }\end{array}$ & $\begin{array}{l}\text { VB16- } \\
\text { 09C }\end{array}$ & $\begin{array}{l}\text { VB16- } \\
\text { 10A }\end{array}$ & $\begin{array}{l}\text { VB16- } \\
\text { 10B }\end{array}$ & $\begin{array}{l}\text { VB16- } \\
10 \mathrm{C}\end{array}$ \\
\hline PCDE-3 (4-monoCI) & 6.2 & 1.3 & & & 20.5 & 268.4 & 2.0 & 32.1 & 32.3 & 34.4 & 818.6 & 10.2 & 116.1 & 0.9 \\
\hline *PCDE-mono-1 & 1.6 & 0.6 & & & 6.8 & 156.6 & & 26.8 & 6.1 & 3.9 & 719.8 & 6.4 & 10.7 & \\
\hline *PCDE-mono-2 & & & & & 0.9 & 6.9 & & 0.9 & 1.0 & & 26.6 & & 1.5 & \\
\hline PCDE-15 (4,4'-diCl) & 26.1 & 6.8 & & & 45.3 & 134.1 & 6.1 & 23.6 & 69.9 & 64.4 & 395.6 & 29.9 & 210.4 & 2.5 \\
\hline *PCDE-di-1/PCDE12 & 7.3 & 3.8 & & & 18.6 & 272.2 & & 20.5 & 27.8 & 37.9 & 812.6 & 8.8 & 90.4 & 2.3 \\
\hline *PCDE-di-2 & 3.7 & 2.9 & & & 16.9 & 198.5 & & 28.8 & 10.4 & 9.7 & 413.8 & 3.9 & 5.2 & \\
\hline *PCDE-di-3 & 2.5 & & & & 3.0 & 7.8 & & 2.4 & 3.7 & 4.4 & 14.2 & 2.7 & 9.6 & \\
\hline *PCDE-di-4 & & & & & & & & & & & & & 2.2 & \\
\hline \multicolumn{15}{|l|}{ *PCDE-di-5 } \\
\hline *PCDE-di-6 & & & & & & 5.6 & & 2.3 & & & 10.2 & & 2.8 & \\
\hline *PCDE-di-7 & & & & & 4.2 & 28.5 & & 7.5 & 4.2 & 3.7 & 203.1 & 3.0 & 4.9 & \\
\hline PCDE-28 $\left(2,4,4^{\prime}\right.$-triCl $)$ & 3.8 & 3.0 & & & 5.6 & 7.3 & & 2.3 & 7.5 & 5.0 & 13.9 & 6.6 & 17.1 & \\
\hline *PCDE-37-tri & 6.3 & 3.5 & & & 10.4 & 39.6 & & 3.3 & 15.0 & 34.9 & 118.4 & 7.7 & 85.9 & \\
\hline *PCDE-tri-1 & 18.3 & 5.5 & & & 67.9 & 331.7 & 4.7 & 40.4 & 49.7 & 41.9 & 621.8 & 14.9 & 44.0 & 2.4 \\
\hline *PCDE-tri-2 & 2.7 & & & & 4.4 & 29.2 & & 7.4 & 5.1 & 4.0 & 84.9 & 2.6 & 5.7 & \\
\hline *PCDE-tri-3 & 2.4 & & & & 2.7 & 11.3 & & 2.7 & 3.7 & 4.1 & 37.4 & 2.4 & 7.5 & \\
\hline *PCDE-tri-4 & & & & & & 2.7 & & & 3.6 & 3.6 & & 7.8 & 3.8 & \\
\hline \multicolumn{15}{|l|}{ *PCDE-tri-5 } \\
\hline *PCDE-tri-6 & & & & & & 3.2 & & & & 2.9 & & & 3.8 & \\
\hline \multicolumn{15}{|l|}{ *PCDE-tri-7 } \\
\hline $\begin{array}{l}\text { PCDE-74 }(2,4,4 ', 5- \\
\text { TetraCl) }\end{array}$ & 27.3 & 7.0 & & & 35.0 & 77.9 & 5.0 & 8.4 & 56.2 & 34.2 & 148.8 & 25.3 & 132.5 & \\
\hline $\begin{array}{l}\text { PCDE-77 }\left(3,3 ', 4,4^{\prime}-\right. \\
\text { TetraCl) }\end{array}$ & 4.6 & & & & 5.4 & 40.5 & & 4.4 & 9.6 & 12.3 & 148.0 & 5.4 & 24.2 & \\
\hline *PCDE-tetra-1 & 7.7 & 3.7 & & & 9.6 & 15.8 & & & 13.8 & 7.9 & 41.4 & 20.1 & 56.7 & 2.9 \\
\hline *PCDE-tetra-2 & 4.5 & 3.4 & & & 9.7 & 11.1 & & 2.6 & 5.9 & 6.1 & 21.7 & 5.8 & 23.1 & \\
\hline *PCDE-tetra-3 & 4.0 & & & & 9.1 & 44.5 & & & 6.7 & 5.8 & 66.7 & 3.3 & 4.5 & \\
\hline *PCDE-tetra-4 & 3.2 & & & & 3.6 & 4.9 & & & 4.9 & 3.4 & 50.3 & 4.9 & 9.7 & \\
\hline *PCDE-tetra-5 & 3.9 & & & & 5.6 & 13.8 & & & 4.9 & 8.0 & 25.7 & 4.6 & 16.3 & \\
\hline *PCDE-tetra-6 & & & & & 3.4 & 5.5 & & & 5.7 & 4.3 & 11.0 & 4.8 & 16.3 & \\
\hline *PCDE-tetra-7 & & & & & & 3.5 & & & & & 4.5 & & 4.3 & \\
\hline *PCDE-tetra-8 & & & & & & 4.6 & & & & & 7.7 & & & \\
\hline $\begin{array}{l}\text { PCDE-99 }\left(2,2^{\prime}, 4,4^{\prime}, 5-\right. \\
\text { PentaCl })\end{array}$ & 41.4 & 9.7 & & & 45.7 & 69.2 & & 3.6 & 74.2 & 41.3 & 79.4 & 74.7 & 271.4 & \\
\hline *PCDE-penta-1 & 3.8 & & & & 4.2 & 5.6 & & & 5.8 & 4.3 & 6.0 & 4.3 & 13.5 & \\
\hline *PCDE-penta-2 & 29.7 & 7.6 & & & 36.3 & 50.8 & 5.2 & 3.7 & 52.7 & 25.5 & 280.6 & 49.5 & 200.0 & 3.1 \\
\hline *PCDE-penta-3_PCDE86 & 4.8 & & & & 5.7 & 20.0 & & 3.1 & 9.4 & 10.2 & 50.6 & 5.2 & 20.0 & \\
\hline
\end{tabular}




\begin{tabular}{|c|c|c|c|c|c|c|c|c|c|c|c|c|c|c|}
\hline Name & $\begin{array}{l}\text { VB16- } \\
\text { 07A }\end{array}$ & $\begin{array}{l}\text { VB16- } \\
\text { 07B }\end{array}$ & $\begin{array}{l}\text { VB16- } \\
\text { 07C }\end{array}$ & $\begin{array}{l}\text { VB16- } \\
\text { 07D }\end{array}$ & $\begin{array}{l}\text { VB16- } \\
\text { 08A }\end{array}$ & $\begin{array}{l}\text { VB16- } \\
\text { 08B }\end{array}$ & $\begin{array}{l}\text { VB16- } \\
\text { 08C }\end{array}$ & $\begin{array}{l}\text { VB16- } \\
\text { 08D }\end{array}$ & $\begin{array}{l}\text { VB16- } \\
\text { 09A }\end{array}$ & $\begin{array}{l}\text { VB16- } \\
\text { 09B }\end{array}$ & $\begin{array}{l}\text { VB16- } \\
\text { 09C }\end{array}$ & $\begin{array}{l}\text { VB16- } \\
\text { 10A }\end{array}$ & $\begin{array}{l}\text { VB16- } \\
\text { 10B }\end{array}$ & $\begin{array}{l}\text { VB16- } \\
\text { 10C }\end{array}$ \\
\hline *PCDE-penta-4 & 3.7 & & & & 4.2 & 8.5 & & & 8.3 & 6.2 & 10.9 & 7.7 & 21.8 & \\
\hline *PCDE-penta-5 & 3.2 & & & & 6.0 & 7.3 & & 2.9 & 5.1 & 3.7 & 91.6 & 3.4 & 4.7 & \\
\hline *PCDE-penta-6 & 3.2 & & & & 3.7 & 8.5 & & & 4.8 & 4.3 & 20.0 & 3.6 & 12.7 & \\
\hline *PCDE-penta-7 & 3.9 & & & & 8.5 & 16.1 & & 3.4 & 8.8 & 4.7 & 48.2 & 3.7 & 7.3 & \\
\hline *PCDE-penta-8 & 4.6 & & & & 4.9 & 6.2 & & & 5.7 & 4.7 & 31.5 & 5.7 & 28.5 & \\
\hline *PCDE-penta-9 & & & & & & & & & & & 4.6 & & 3.9 & \\
\hline *PCDE-penta-10 & & & & & & 4.8 & & & 3.3 & 3.5 & 7.3 & & 5.1 & \\
\hline *PCDE-penta-11 & & & & & & 3.4 & & & & & 4.8 & & 4.0 & \\
\hline *PCDE-penta-12 & & & & & & & & & & & 4.8 & & 3.9 & \\
\hline *PCDE141 & 7.9 & & & & 12.2 & 20.8 & & & 17.7 & 11.6 & 28.5 & 14.9 & 58.7 & \\
\hline *PCDE-hexa-1 & 45.6 & 10.1 & & & 69.7 & 114.9 & 5.2 & 4.9 & 101.7 & 53.8 & 222.5 & 74.3 & 326.2 & \\
\hline *PCDE-hexa-2 & 11.0 & 4.4 & & & 20.6 & 38.1 & & 3.3 & 24.8 & 14.3 & 75.9 & 15.2 & 61.1 & \\
\hline *PCDE-hexa-3 & 5.3 & 3.6 & & & 7.1 & 17.8 & & & 11.5 & 14.6 & 27.7 & 6.5 & 29.5 & \\
\hline *PCDE-hexa-4 & 28.2 & 7.5 & & & 68.6 & 158.6 & & 7.0 & 97.0 & 64.6 & 311.6 & 58.1 & 251.0 & 2.8 \\
\hline *PCDE-hexa-5 & 18.6 & 5.1 & & & 18.8 & 47.4 & & 3.2 & 45.9 & 22.7 & 69.6 & 41.9 & 50.0 & \\
\hline *PCDE-hexa-6 & 4.0 & & & & 5.5 & 8.2 & & & 7.5 & 5.5 & 11.7 & 7.4 & 26.4 & \\
\hline *PCDE-hexa-7 & 11.3 & 3.8 & & & 13.1 & 29.8 & & & 25.9 & 16.9 & 39.7 & 23.2 & 89.5 & \\
\hline *PCDE-hexa-8 & & & & & & 10.6 & & & 4.7 & 4.3 & 22.6 & & 7.8 & \\
\hline *PCDE-hexa-9 & & & & & & & & & & & 4.1 & & 4.3 & \\
\hline *PCDE-hexa-10 & & & & & & & & & & & 6.0 & & & \\
\hline $\begin{array}{l}\text { PCDE-180 }\left(2,2^{\prime}, 3,4,4^{\prime}, 5,5^{\prime}-\right. \\
\text { HeptaCl) }\end{array}$ & 4.2 & & & & 9.8 & 34.5 & 3.8 & 2.2 & 17.0 & 10.5 & 78.8 & 10.8 & 42.4 & \\
\hline *PCDE-hepta-1 & 43.1 & 7.0 & & & 83.7 & 195.2 & & 4.8 & 110.4 & 67.2 & 366.6 & 76.4 & 356.5 & \\
\hline *PCDE-hepta-2 & 18.0 & 3.6 & & & 43.5 & 82.7 & & 3.1 & 51.3 & 30.1 & 170.8 & 32.9 & 159.1 & \\
\hline *PCDE-hepta-3 & 3.6 & & & & 5.4 & 12.8 & & & 5.1 & 4.7 & 24.7 & 4.9 & 24.6 & \\
\hline *PCDE-hepta-4 & 13.0 & & & & 29.6 & 64.2 & & 2.8 & 36.5 & 23.5 & 119.8 & 200.0 & 119.4 & \\
\hline *PCDE-hepta-5 & & & & & 2.9 & 5.3 & & & & & 14.4 & & 5.7 & \\
\hline *PCDE-hepta-6 & & & & & 3.8 & 6.6 & & & & & 14.2 & 3.5 & 11.7 & \\
\hline *PCDE-hepta-7 & & & & & & & & & & & 5.2 & & 2.8 & \\
\hline *PCDE-octa-1 & 5.8 & & & & 24.0 & 45.6 & & 3.3 & 21.7 & 12.1 & 109.1 & 9.1 & 55.2 & \\
\hline *PCDE-octa-2 & 2.6 & & & & 4.9 & 7.9 & & & 3.9 & 3.5 & 20.2 & 2.9 & 11.9 & \\
\hline *PCDE-octa-3 & & & & & 3.7 & 5.9 & & & 4.3 & 3.2 & 20.3 & 2.8 & 10.6 & \\
\hline *PCDE-octa-4 & & & & & 3.0 & 4.8 & & & & 2.7 & 7.3 & & 4.3 & \\
\hline *PCDE-octa-5 & & & & & & & & & & & 4.7 & & 2.7 & \\
\hline *PCDE-nona-1 & & & & & 3.3 & 6.8 & & & 2.6 & & 16.8 & & 6.3 & \\
\hline
\end{tabular}

Note: samples are reported as $\mathrm{ng} / \mathrm{g}$ 


\begin{tabular}{|c|c|c|c|c|c|c|c|c|c|c|c|c|c|c|}
\hline Name & $\begin{array}{c}\text { VB16- } \\
\text { 07A }\end{array}$ & $\begin{array}{c}\text { VB16- } \\
\text { 07B }\end{array}$ & $\begin{array}{c}\text { VB16- } \\
\text { 07C }\end{array}$ & $\begin{array}{c}\text { VB16- } \\
\text { 07D }\end{array}$ & $\begin{array}{c}\text { VB16- } \\
\text { 08A }\end{array}$ & $\begin{array}{c}\text { VB16- } \\
\text { 08B }\end{array}$ & $\begin{array}{c}\text { VB16- } \\
\text { 08C }\end{array}$ & $\begin{array}{c}\text { VB16- } \\
\text { 08D }\end{array}$ & $\begin{array}{c}\text { VB16- } \\
\text { 09A }\end{array}$ & $\begin{array}{c}\text { VB16- } \\
\text { 09B }\end{array}$ & $\begin{array}{c}\text { VB16- } \\
\text { 09C }\end{array}$ & $\begin{array}{c}\text { VB16- } \\
\text { 10A }\end{array}$ & $\begin{array}{c}\text { VB16- } \\
\text { 10B }\end{array}$ & $\begin{array}{c}\text { VB16- } \\
10 \mathrm{C}\end{array}$ \\
\hline Total PCDE Calculations & $\begin{array}{c}\text { VB16- } \\
\text { 07A }\end{array}$ & $\begin{array}{c}\text { VB16- } \\
\text { 07B }\end{array}$ & $\begin{array}{l}\text { VB16- } \\
\text { 07C }\end{array}$ & $\begin{array}{c}\text { VB16- } \\
\text { 07D }\end{array}$ & $\begin{array}{c}\text { VB16- } \\
\text { 08A }\end{array}$ & $\begin{array}{c}\text { VB16- } \\
\text { 08B }\end{array}$ & $\begin{array}{c}\text { VB16- } \\
\text { 08C }\end{array}$ & $\begin{array}{c}\text { VB16- } \\
\text { 08D } \\
\end{array}$ & $\begin{array}{c}\text { VB16- } \\
\text { 09A }\end{array}$ & $\begin{array}{c}\text { VB16- } \\
\text { 09B }\end{array}$ & $\begin{array}{c}\text { VB16- } \\
\text { 09C }\end{array}$ & $\begin{array}{c}\text { VB16- } \\
\text { 10A }\end{array}$ & $\begin{array}{c}\text { VB16- } \\
\text { 10B }\end{array}$ & $\begin{array}{c}\text { VB16- } \\
\text { 10C }\end{array}$ \\
\hline Total mono-Cl-PCDE & 7.8 & 2.0 & & & 28.2 & 431.9 & 2.0 & 59.8 & 39.4 & 38.3 & 1565.0 & 16.7 & 128.2 & 0.9 \\
\hline Total di-Cl-PCDE & 39.6 & 13.6 & & & 88.1 & 646.7 & 6.1 & 85.1 & 116.1 & 120.0 & 1849.5 & 48.2 & 325.5 & 4.7 \\
\hline Total tri-Cl-PCDE & 33.4 & 12.0 & & & 90.9 & 425.0 & 4.7 & 56.1 & 84.5 & 96.3 & 876.4 & 41.9 & 167.8 & 2.4 \\
\hline Total tetra-Cl-PCDE & 55.2 & 14.2 & & & 81.4 & 222.0 & 5.0 & 15.5 & 107.6 & 82.0 & 525.8 & 74.1 & 287.4 & 2.9 \\
\hline Total penta-Cl-PCDE & 98.3 & 17.3 & & & 119.0 & 200.3 & 5.2 & 16.7 & 178.2 & 108.4 & 640.1 & 157.9 & 596.6 & 3.1 \\
\hline Total hexa-Cl-PCDE & 131.8 & 34.5 & & & 215.6 & 446.2 & 5.2 & 18.4 & 336.8 & 208.1 & 819.7 & 241.6 & 904.4 & 2.8 \\
\hline Total hepta-Cl-PCDE & 81.9 & 10.6 & & & 178.6 & 401.3 & 3.8 & 13.0 & 220.3 & 136.1 & 794.6 & 328.5 & 722.1 & \\
\hline Total octa-Cl-PCDE & 8.4 & & & & 35.5 & 64.1 & & 3.3 & 29.9 & 21.5 & 161.7 & 14.8 & 84.6 & \\
\hline Total nona-Cl-PCDE & & & & & 3.3 & 6.8 & & & 2.6 & & 16.8 & & 6.3 & \\
\hline Total PCDE (ng/g) & 456.3 & 104.0 & ND & ND & 840.6 & 2844.3 & 32.0 & 267.7 & 1115.3 & 810.6 & 7249.5 & 923.6 & 3222.8 & 16.8 \\
\hline
\end{tabular}

\begin{tabular}{|c|c|c|c|c|c|c|c|}
\hline Name & VB16-12A & VB16-12B & VB16-12C & VB16-12D & VB16-13A & VB16-13B & VB16-13C \\
\hline PCDE-3 (4-monoCI) & 142.8 & 108.5 & 43.6 & 0.7 & 7.0 & 11.1 & 0.6 \\
\hline *PCDE-mono-1 & 314.4 & 264.3 & 29.3 & 0.8 & 0.7 & 1.1 & \\
\hline *PCDE-mono-2 & 4.9 & 3.9 & 0.9 & & & & \\
\hline PCDE-15 (4,4'-diCl) & 130.0 & 91.0 & 47.4 & 2.2 & 17.0 & 25.1 & \\
\hline *PCDE-di-1/PCDE12 & 220.5 & 209.3 & 52.9 & 2.5 & 4.9 & 7.6 & \\
\hline *PCDE-di-2 & 475.5 & 252.1 & 27.4 & 2.6 & 2.5 & 3.7 & \\
\hline *PCDE-di-3 & 6.4 & 3.7 & 3.5 & & 2.4 & 2.7 & \\
\hline *PCDE-di-4 & 4.7 & 2.6 & & & & & \\
\hline \multicolumn{8}{|l|}{ *PCDE-di-5 } \\
\hline *PCDE-di-6 & 3.5 & 2.7 & & & & & \\
\hline *PCDE-di-7 & 74.1 & 48.3 & 12.6 & 2.5 & 2.1 & 2.9 & \\
\hline PCDE-28 $(2,4,4$ '-triCl) & 9.4 & 6.7 & 4.1 & & 2.7 & 2.8 & \\
\hline *PCDE-37-tri & 26.9 & 15.5 & 15.2 & & 5.4 & 8.6 & \\
\hline *PCDE-tri-1 & 456.3 & 136.4 & 35.7 & 2.3 & 10.2 & 13.1 & \\
\hline *PCDE-tri-2 & 74.0 & 22.2 & 6.2 & & 2.5 & 2.6 & \\
\hline *PCDE-tri-3 & 8.0 & 9.3 & 4.5 & & & 2.3 & \\
\hline *PCDE-tri-4 & & & & & & 2.4 & \\
\hline *PCDE-tri-5 & 4.2 & & 3.1 & & & & \\
\hline *PCDE-tri-6 & 3.1 & & & & & & \\
\hline *PCDE-tri-7 & 3.9 & & & & & & \\
\hline PCDE-74 $(2,4,4 ', 5$-TetraCl $)$ & 177.6 & 51.2 & 15.7 & & 11.5 & 15.0 & \\
\hline PCDE-77 $\left(3,3^{\prime}, 4,4^{\prime}\right.$-TetraCl) & 35.3 & 32.2 & 12.2 & & 3.8 & 4.0 & \\
\hline
\end{tabular}




\begin{tabular}{|c|c|c|c|c|c|c|c|}
\hline Name & VB16-12A & VB16-12B & VB16-12C & VB16-12D & VB16-13A & VB16-13B & VB16-13C \\
\hline *PCDE-tetra-1 & 35.5 & 13.5 & 5.3 & & 3.5 & 4.9 & \\
\hline *PCDE-tetra-2 & 28.3 & 6.7 & 3.8 & & 3.2 & 4.0 & \\
\hline *PCDE-tetra-3 & 145.1 & 13.9 & 4.9 & & 3.0 & & \\
\hline *PCDE-tetra-4 & 16.2 & 6.1 & & & & & \\
\hline *PCDE-tetra-5 & 13.7 & 4.2 & 3.9 & & 4.2 & 3.1 & \\
\hline *PCDE-tetra-6 & 8.6 & 4.8 & 3.9 & & 3.3 & 3.1 & \\
\hline *PCDE-tetra-7 & 6.2 & 3.8 & & & & & \\
\hline *PCDE-tetra-8 & 6.5 & 3.1 & & & & & \\
\hline PCDE-99 $\left(2,2^{\prime}, 4,4^{\prime}, 5\right.$-PentaCI) & 72.9 & 46.5 & 13.2 & & 12.6 & 14.6 & \\
\hline *PCDE-penta-1 & 6.7 & 4.9 & & & 3.1 & 3.2 & \\
\hline *PCDE-penta-2 & 66.4 & 27.5 & 9.2 & & 9.9 & 11.1 & \\
\hline *PCDE-penta-3_PCDE86 & 15.8 & 10.3 & 5.4 & & 3.1 & 3.4 & \\
\hline *PCDE-penta-4 & 8.1 & 5.0 & 4.3 & & 3.1 & 3.2 & \\
\hline *PCDE-penta-5 & 11.2 & 4.5 & 4.0 & & 3.2 & & \\
\hline *PCDE-penta-6 & 8.1 & 4.9 & 4.2 & & & 3.1 & \\
\hline *PCDE-penta-7 & 28.8 & 8.5 & 4.6 & & & 3.2 & \\
\hline *PCDE-penta-8 & 7.4 & 4.4 & & & & & \\
\hline *PCDE-penta-9 & 3.8 & 2.8 & & & & & \\
\hline *PCDE-penta-10 & 3.7 & 3.0 & & & & & \\
\hline *PCDE-penta-11 & 3.3 & & & & & & \\
\hline *PCDE-penta-12 & 3.5 & 3.1 & & & & & \\
\hline *PCDE141 & 17.6 & 9.9 & 5.4 & & 3.5 & 4.1 & \\
\hline *PCDE-hexa-1 & 151.4 & 52.6 & 14.7 & & 13.2 & 16.9 & \\
\hline *PCDE-hexa-2 & 47.3 & 17.0 & 6.5 & & 4.2 & 5.8 & \\
\hline *PCDE-hexa-3 & 15.3 & 7.8 & 4.5 & & 3.3 & 3.7 & \\
\hline *PCDE-hexa-4 & 194.8 & 69.7 & 10.3 & & 11.8 & 17.0 & \\
\hline *PCDE-hexa-5 & 46.0 & 24.4 & 9.4 & & 6.5 & 8.3 & \\
\hline *PCDE-hexa-6 & 8.3 & 5.3 & & & 3.1 & 3.2 & \\
\hline *PCDE-hexa-7 & 22.0 & 14.2 & 6.4 & & 4.4 & 4.8 & \\
\hline *PCDE-hexa-8 & 50.0 & 3.9 & & & & & \\
\hline \multicolumn{8}{|l|}{ *PCDE-hexa-9 } \\
\hline *PCDE-hexa-10 & 3.5 & & & & & & \\
\hline $\begin{array}{l}\text { PCDE-180 }\left(2,2^{\prime}, 3,4,4^{\prime}, 5,5^{\prime}-\right. \\
\text { HeptaCl) }\end{array}$ & 29.8 & 12.6 & 4.3 & & 3.0 & 4.0 & \\
\hline *PCDE-hepta-1 & 144.8 & 36.7 & 17.9 & & 8.1 & 15.0 & \\
\hline *PCDE-hepta-2 & 78.3 & 31.8 & 7.1 & & 3.7 & 9.4 & \\
\hline *PCDE-hepta-3 & 9.2 & 5.4 & 3.1 & & & & \\
\hline
\end{tabular}




\begin{tabular}{|c|c|c|c|c|c|c|c|}
\hline Name & VB16-12A & VB16-12B & VB16-12C & VB16-12D & VB16-13A & VB16-13B & VB16-13C \\
\hline *PCDE-hepta-4 & 43.2 & 21.9 & 8.3 & & 4.0 & 5.9 & \\
\hline *PCDE-hepta-5 & 5.7 & 3.0 & & & & & \\
\hline *PCDE-hepta-6 & 4.1 & 3.5 & & & & & \\
\hline *PCDE-hepta-7 & 3.5 & 2.4 & & & & & \\
\hline *PCDE-octa-1 & 62.7 & 23.5 & 6.2 & & 2.8 & 2.8 & \\
\hline *PCDE-octa-2 & 6.6 & 4.7 & & & & & \\
\hline *PCDE-octa-3 & 11.5 & 3.6 & & & & & \\
\hline *PCDE-octa-4 & 6.6 & 3.3 & & & & & \\
\hline *PCDE-octa-5 & 3.6 & & & & & & \\
\hline *PCDE-nona-1 & 8.5 & 2.9 & & & & & \\
\hline
\end{tabular}

Note: samples are reported as $\mathbf{n g} / \mathrm{g}$

\begin{tabular}{|l|c|c|c|c|c|c|c|}
\hline Total PCDE Calculations & VB16-12A & VB16-12B & VB16-12C & VB16-12D & VB16-13A & VB16-13B & VB16-13C \\
\hline Total mono-Cl-PCDE & 462.1 & 376.7 & 73.8 & 1.5 & 7.8 & 12.1 & 0.6 \\
\hline Total di-Cl-PCDE & 914.6 & 609.7 & 143.8 & 9.8 & 28.9 & 41.9 & \\
\hline Total tri-Cl-PCDE & 585.7 & 190.0 & 68.7 & 2.3 & 20.8 & 31.8 & \\
\hline Total tetra-Cl-PCDE & 473.0 & 139.5 & 49.5 & & 32.4 & 34.1 & \\
\hline Total penta-Cl-PCDE & 239.7 & 125.3 & 44.9 & & 35.0 & 41.6 & \\
\hline Total hexa-Cl-PCDE & 555.9 & 204.7 & 57.2 & & 49.9 & 63.6 & \\
\hline Total hepta-Cl-PCDE & 318.6 & 117.4 & 40.6 & & 18.8 & 34.3 & \\
\hline Total octa-Cl-PCDE & 91.0 & 35.0 & 6.2 & & 2.8 & 2.8 & \\
\hline Total nona-Cl-PCDE & 8.5 & 2.9 & & & & $\mathbf{1 9 6 . 2}$ & $\mathbf{2 6 2 . 2}$ \\
\hline Total PCDE $(\mathbf{n g} / \mathbf{g})$ & $\mathbf{3 6 4 9 . 1}$ & $\mathbf{1 8 0 1 . 1}$ & $\mathbf{4 8 4 . 7}$ & $\mathbf{1 3 . 5}$ & $\mathbf{0 . 6}$ \\
\hline
\end{tabular}

